\title{
Funktionelle Polymere mittels kontrollierter Polymerisationstechniken: Von der Massenspektrometrie endfunktionalisierter Polymere und den mechanischen Eigenschaften photovernetzbarer Blockcopolymere
}

\author{
Dissertation \\ zur Erlangung des mathematisch-naturwissenschaftlichen Doktorgrades \\ "Doctor rerum naturalium" \\ der Georg-August-Universität Göttingen \\ im Promotionsprogramm Chemie \\ der Georg-August University School of Science
}

(GAUSS)

vorgelegt von

Byron Helmut Staudt

aus Freudenberg

Göttingen, 2019 


\section{Betreuungsausschuss}

Prof. Dr. Philipp Vana, MBA

Institut für Physikalische Chemie

Georg-August-Universität Göttingen

Prof. Dr. Marcus Müller

Institut für Theoretische Physik

Georg-August-Universität Göttingen

\section{Mitglieder der Prüfungskommission}

\section{Referent}

Prof. Dr. Philipp Vana, MBA

Institut für Physikalische Chemie

Georg-August-Universität Göttingen

\section{Koreferent}

Prof. Dr. Marcus Müller

Institut für Theoretische Physik

Georg-August-Universität Göttingen

\section{Weitere Mitglieder der Prüfungskommission}

Prof. Dr. Michael Buback

Prof. Dr. Burkhard Geil

PD Dr. Thomas Zeuch

Prof. Dr. Konrad Koszinowski

Tag der mündlichen Prüfung:
Institut für Physikalische Chemie Georg-August-Universität Göttingen

Institut für Physikalische Chemie Georg-August-Universität Göttingen

Institut für Physikalische Chemie Georg-August-Universität Göttingen

Institut für Organische und Biomolekulare Chemie Georg-August-Universität Göttingen

29.01 .2019 
Wer noch nie einen Fehler gemacht hat, hat sich noch nie an etwas Neuem versucht.

- Albert Einstein 



\section{Zusammenfassung}

In dieser Arbeit wurden Polymere mit terminalen Ammoniumgruppen funktionalisiert, wodurch sich neue Anwendungsmöglichkeiten ergeben und die Analyse mittels Massenspektrometrie ermöglicht beziehungsweise optimiert wird. Zudem wurden reversibel photovernetzbare Blockcopolymere synthetisiert und der Einfluss der Vernetzung auf die mechanischen Eigenschaften untersucht.

lodendfunktionalisiertes Polyethylen (PE) mit niedriger Dispersität wurde mittels catalyzed chain growth Polymerisation hergestellt. Durch eine quantitative nucleophile Substitution konnte eine tertiäre Amino- beziehungsweise Ammoniumendgruppe erfolgreich eingeführt und das PE mittels Gelpermeationschromatographie und Kernspinresonanzspektroskopie charakterisiert werden. Darüber hinaus ermöglichten beide polaren Endgruppen nach Optimierung der Probenvorbereitung die massenspektrometrische Analyse der Molmassenverteilung und der Endgruppen von PE, was aufgrund mangelnder polarer Gruppen sonst nicht möglich ist. Dabei wurden sowohl durch die matrix-assisted laser desorption ionization(MALDI) als auch durch die Elektrospray-lonisations-Massenspektrometrie (ESI-MS) Massenspektren mit exzellentem Signal-zu-Rausch(S/R)-Verhältnissen erhalten. Des Weiteren ermöglichte die Ammoniumgruppe die Verwendung des PE als einfach abzutrennenden und recycelbaren Phasentransferkatalysator (PTK), der in einer Modellreaktion eine mit kommerziell etablierten PTK vergleichbare oder sogar höhere Effektivität aufwies. Aufgrund der Phasentransfereigenschaften dieses PE konnte ein anionischer Farbstoff aus der wässrigen Phase extrahiert und in festem PE durch starke ionische Wechselwirkungen eingebaut werden. Zudem war durch eine zusätzlich vorhandene Hydroxylgruppe weitere Funktionalisierung des geladenen PE möglich. So gelang es ein geladenes PE-basiertes Makromonomer und einen geladenen Makroinitiator für die Atom-Transfer radikalische Polymerisation herzustellen. Letzterer wurde erfolgreich für die Herstellung eines Blockcopolymers verwendet.

Polystyrol (PS) und Polymethyacrylat (PMA) wurden mit und ohne ionische Endgruppen mittels reversible addition-fragmentation chain transfer (RAFT) Polymerisation hergestellt und anschließend massenspektrometrisch analysiert. Das S/R-Verhältnis und die Signalintensitäten der Massenspektren von Polymeren mit geladener Endgruppe wurden im Vergleich zu denen ohne Ammoniumgruppe sowohl in der ESI- als auch in der MALDI-MS maßgeblich erhöht. Mit zwei geladene Gruppen in der Polymerkette war es möglich, die Anzahl der Ladungen pro Kette zu kontrollieren und gezielt zweifach geladene Ketten auch bei sehr kleinen Polymerisationsgraden in der ESI-MS zu erzeugen. In der MALDI-MS war dies durch Neutralisation einer Ammoniumgruppe mit einem Gegenion nicht möglich, weshalb nur einfach geladene Ketten entstanden. Es traten dort auch weniger Spezies auf als in der ESI-MS. Generell konnten aber nahezu alle mit beiden lonisationsverfahren detektierten Spezies einer Struktur zugewiesen werden. In der ESI-MS wurden zudem der Einfluss mehrerer Mess- beziehungsweise Geräteparameter untersucht: Durch die Verwendung von 
Nal für die lonisation der PS- und PMA-Ketten entstanden in der ESI-MS ab mindestens fünf Äquivalenten Natriumiodid $\mathrm{Na}(\mathrm{Nal})_{\mathrm{X}}$-Cluster und durch Addukte mit $\mathrm{Na}(\mathrm{Nal})_{\mathrm{X}}$ ionisierte Polymerketten. Durch diese Vielzahl neuer Signale wurde die Interpretation der Massenspektren erschwert. Es konnte gezeigt werden, dass eine niedrigere Kapillarspannung die Fragmentierung der labilen Trithiocarbonatgruppe erschwert. Durch Einstellung der Stoßenergie im ESI-Massenspektrometer konnte in PMA mit geladener Endgruppe die mittlere Ladungsanzahl der Ketten stark reduziert werden. Dies führt zu Massenspektren, die leichter zu interpretieren sind.

Schließlich wurden erfolgreich reversibel photovernetzbare Blockcopolymere (BCP) durch die RAFT Polymerisation und den Einbau eines Anthracenmonomers (ANMA) hergestellt. In statistischen Copolymeren aus ANMA und Laurylmethacrylat (LMA) konnte die über mehrere Zyklen nahezu vollständige Photovernetzung und thermische Netzwerkspaltung mittels Absorptionsspektroskopie in dünnen Filmen nachgewiesen werden. Zusätzlich wurde der Einfluss der Photovernetzung innerhalb dieser Copolymeren auf die mittels Zugversuch bestimmten mechanischen Eigenschaften in bulk untersucht. Dabei stellte sich heraus, dass die Zugfestigkeit und vor allem der E-Modul durch die Vernetzung auch bei nur kleinen Anteilen von ANMA enorm erhöht werden können, wohingegen die Bruchdehnung abnimmt. Der Anteil an vernetztem Material wurde in Abhängigkeit des ANMA-Gehaltes bestimmt. Hierbei zeigte sich, dass bei geringeren Konzentrationen von ANMA mehr Material vernetzt werden konnte. Zudem wurde gezeigt, dass die thermische Netzwerkspaltung und eine erneute Photovernetzung in bulk möglich sind, wobei die mechanischen Eigenschaften im Wesentlichen beibehalten werden. Die hergestellten BCP bestehend aus einem harten Butylmethacrylat- (BMA) und einem weichen LMA-Block enthielten in jeweils einem der beiden Blöcke ANMA. Anhand der Rasterkraftmikroskopie konnte Mikrophasenseparation mit einer weichen und einer harten Phase nachgewiesen werden. Erstmalig wurde der Einfluss der selektiven Photovernetzung innerhalb der weichen oder harten Phase auf die mechanischen Eigenschaften des Gesamtprobenkörpers untersucht. Dabei zeigte sich, dass die Vernetzung der LMA-Phase die gleichen Auswirkungen auf die mechanischen Eigenschaften zufolge hat wie die Vernetzung der oben beschriebenen statistischen Copolymere. Die Vernetzung der BMA-Phase bewirkt nahezu keine Veränderung des E-Moduls, jedoch nimmt die Bruchdehnung ab. 


\section{Inhaltsverzeichnis}

Abkürzungsverzeichnis $\ldots \ldots \ldots \ldots \ldots \ldots \ldots \ldots \ldots \ldots \ldots \ldots \ldots \ldots$

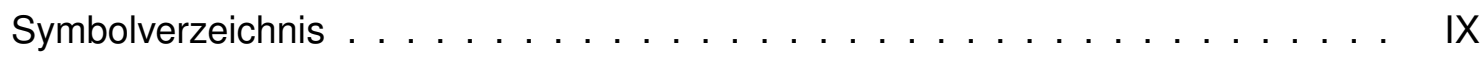

Einheitenverzeichnis . . . . . . . . . . . . . . . . . XI

\begin{tabular}{lll}
\hline 1 & Einleitung & 1
\end{tabular}

2 Theorie und Kenntnisstand 3

2.1 Kontrollierte reversibel-desaktivierende radikalische Polymerisationstechniken 3

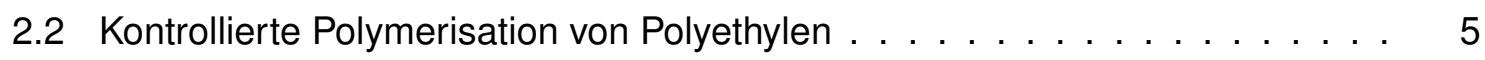

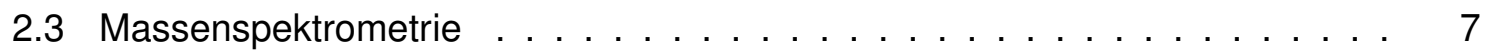

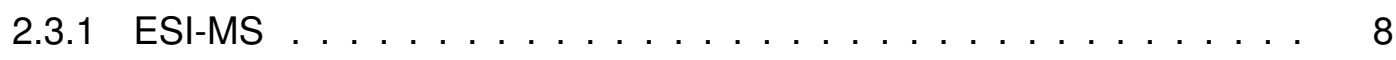

2.3 .2 MALDI-MS $\ldots \ldots \ldots \ldots \ldots \ldots$

2.3 .3 Massenspektrometrie von Polymeren . . . . . . . . . . . 12

2.3 .4 Ladungsreduzierung der Polymerketten in der ESI-MS . . . . . . . . 13

2.3 .5 Massenspektrometrie von Polyethylen . . . . . . . . . . . 14

2.4 Blockcopolymere . . . . . . . . . . . . . . . . . . . . 15

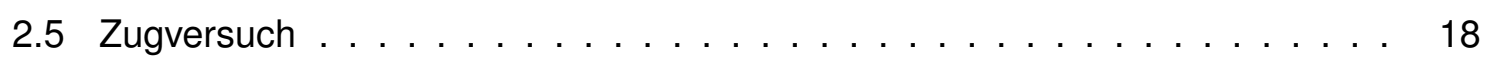

2.6 Reversible Photovernetzung . . . . . . . . . . . . . . . . . . 19

3 Kationische Endgruppen in PE 23

3.1 Motivation . . . . . . . . . . . . . . . . . . . . . 23

3.2 Synthese und Charakterisierung von PE mit tertiären Amin- und Ammoniu-

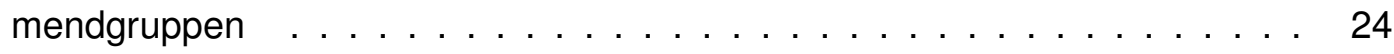

3.3 Phasentransfereigenschaften und Beladung von PE mit anionischen, organischen Verbindungen $\ldots \ldots \ldots \ldots \ldots \ldots \ldots \ldots \ldots \ldots$

3.4 Verwendung von kationischem PE als Phasentransferkatalysator . . . . . 32

3.5 Weitere Funktionalisierung von PE- $\mathrm{N}^{+}$und $\mathrm{PE}-1 \ldots \ldots \ldots \ldots \ldots$

3.5 .1 Weitere Funktionalisierungen mittels nucleophiler Substitution . . . 35

3.5.2 Weitere Funktionalisierungen durch Veresterung der Hydroxylgruppe 37

3.6 Blockcopolymerisation von PE- $\mathrm{N}^{+}$-ATRP mit Butylacrylat $\ldots \ldots \ldots \ldots$

3.7 Schlussfolgerung und Ausblick $\ldots \ldots \ldots \ldots \ldots \ldots \ldots$. . . . . . . 42

4 Kationische Endgruppen in RAFT-Polymeren 43

4.1 Motivation . . . . . . . . . . . . . . . . . . . . . . . 43

4.2 Synthese und Charakterisierung der RAFT-Agenzien $\ldots \ldots \ldots \ldots .44$ 
4.3 Synthese der RAFT-Polymere . . . . . . . . . . . . . . . . . . . . . 45

4.4 ESI-MS Analyse der RAFT-Polymere . . . . . . . . . . . . . . . . . . . . . 50

$4.4 .1 \quad$ Bestimmung der detektierten Spezies der Polymere . . . . . . . . . . 50

4.4 .2 Einfluss der Natriumkonzentration . . . . . . . . . . . . . . . . . 61

4.4 .3 Einfluss der Kapillarspannung. . . . . . . . . . . . . . . . . . . . 64

4.4 .4 Einfluss der Flussrate . . . . . . . . . . . . . . . . . . . . . . 68

4.4.5 Einfluss der einfach und zweifach geladenen ionischen Endgruppe . . 70

4.4.6 Einfluss der Stoßenergie auf mehrfach geladenen Polymerketten in der ESI-MS . . . . . . . . . . . . . . . . . . . 73

4.5 MALDI-MS Analyse der RAFT-Polymere . . . . . . . . . . . . . . . . . . 77

4.5 .1 Analyse der Polymethylacrylate . . . . . . . . . . . . . . . . . . . . . . . . . . . . . . . . . . . . . . . .

4.5 .2 Analyse der Polystyrole . . . . . . . . . . . . . . . . . . . . . . . . . . . . . . . . . 85

4.6 Schlussfolgerung und Ausblick $\ldots \ldots \ldots \ldots$. . . . . . . . . . . . . . . 87

5 Mechanische Eigenschaften reversibel photovernetzbarer Blockcopolymere 89

5.1 Motivation . . . . . . . . . . . . . . . . . . . . . . . . . 89

5.2 Verwendung von Cumarin als Photovernetzer . . . . . . . . . . . . . . . . 90

5.2 .1 Synthese des Cumarinmonomers . . . . . . . . . . . . . . . . 90

5.2 .2 Synthese der photovernetzbaren cumarinhaltigen Polymere . . . . . . 90

5.2 .3 Bestimmung der Morphologie der Mikrophasenseparation . . . . . . 93

5.2 .4 Untersuchung des Ordnungs-Unordnungs-Übergangs . . . . . . . . . 95

5.2 .5 Untersuchung der reversiblen Photovernetzung in dünnen Filmen . . 96

5.3 Verwendung von Anthracen als Photovernetzer . . . . . . . . . . . . . . 98

5.3 .1 Synthese des Anthracenmonomers . . . . . . . . . . . . . . . . . 98

5.3 .2 Synthese der photovernetzbaren anthracenhaltigen Polymere . . . . 98

5.3.3 Untersuchung der Blockcopolymere mittels Rasterkraftmikroskopie . 103

5.3.4 Untersuchung der reversiblen Photovernetzung in dünnen Filmen . . 107

5.3 .5 Bestimmung der Glasübergangstemperaturen . . . . . . . . . . . . 108

5.3.6 Untersuchung des Einflusses der Photovernetzung auf die mechanischen Eigenschaften der statistischen Copolymere . . . . . . . . . . . 112

5.3.7 Untersuchung des Einflusses der Photovernetzung auf die mechanischen Eigenschaften der Blockcopolymere . . . . . . . . . . . . 117

5.4 Schlussfolgerung und Ausblick $\ldots \ldots \ldots \ldots \ldots$. . . . . . . . . . . 119

$\begin{array}{lll}6 & \text { Experimentalteil } & 121\end{array}$

6.1 Materialien . . . . . . . . . . . . . . . . . . . . . . . . . . . 121

6.2 Verwendete Geräte und Probenherstellung . . . . . . . . . . . . . . . . . 121

6.3 Synthesen und Versuche: Projekt ionische Endgruppen . . . . . . . . . . . . 126 
\begin{tabular}{ll}
\hline Literatur & 136
\end{tabular}

\begin{tabular}{ll}
\hline Anhang & 145
\end{tabular} 


\section{Abkürzungsverzeichnis}

\begin{tabular}{|c|c|}
\hline Abb. & Abbildung \\
\hline AFM & Rasterkraftmikroskopie (atomic force microscopy) \\
\hline AIBN & Azobis(isobutyronitril) \\
\hline ANMA & 9-Anthracenylmethylmethacrylat \\
\hline äq & Äquivalent \\
\hline arb & willkürlichen (engl. arbitrary) \\
\hline ATRP & Atom-Transfer radikalische Polymerisation \\
\hline $\mathrm{BCP}$ & Blockcopolymer \\
\hline bzw. & beziehungsweise \\
\hline ca. & circa / ungefähr \\
\hline CCG & catalyzed chain growth \\
\hline CDSPA & 4-Cyano-4-[(dodecylsulfanylthiocarbonyl)sulfanyl]pentansäure \\
\hline CEM & chain ejection Modell \\
\hline CMA & 7-(2-Methacryloyloxythoxy)-4-methylcumarin \\
\hline $\mathrm{Cp}{ }^{*}$ & Tetramethylyclopentadienyl-Anion \\
\hline CRM & charged residue Modell \\
\hline DCC & Dicyclohexylcarbodiimid \\
\hline DCE & 1,2-Dichlorethan \\
\hline DCM & Dichlormethan \\
\hline DCTB & trans-2-[3-(4-tert-Butylphenyl)-2-methyl-2-propenyliden]propandinitril \\
\hline d.h. & das heißt \\
\hline DMA & dynamisch-mechanische Analyse \\
\hline DMAP & 4-(Dimethylamino)-pyridin \\
\hline DMF & Dimethylformamid \\
\hline DSC & dynamische Differenzkalorimetrie (dynamic difference calorimetry) \\
\hline ESI & Elektrospray-Ionisation \\
\hline FRP & freie radikalische Polymerisation \\
\hline GPC & Gelpermeationschromatographie, Größenausschluss-Chromatographie \\
\hline HABA & 2-(4-Hydroxyphenylazo)benzoesäure \\
\hline HT & Hochtemperatur \\
\hline I & Initiator \\
\hline IEM & ion evaporation Modell \\
\hline KRP & kontrollierte radikalische Polymerisation \\
\hline M & Monomer \\
\hline MALDI & matrix-assisted laser desorption ionization \\
\hline $\mathrm{MeCN}$ & Acetonitril \\
\hline $\mathrm{MeOH}$ & Methanol \\
\hline MMV & Molmassenverteilung \\
\hline $\mathrm{MO}$ & Methylorange \\
\hline MPS & Mikrophasenseparation \\
\hline MS & Massenspektrometrie \\
\hline
\end{tabular}




$\begin{array}{ll}\text { Nd:YAG } & \text { Neodym-dotierter Yttrium-Aluminium-Granat } \\ \text { NMP } & \text { Nitroxid-vermittelte Polymerisation (nitroxide-mediated polymerization) } \\ \text { NMR } & \text { Kernspinresonanz (nuclear magnetic resonance) } \\ \text { ODT } & \text { Ordnungs-Unordnungs-Übergang (order-disorder-transition) } \\ \text { (P)BA } & \text { (Poly)Butylacrylat } \\ \text { (P)BMA } & \text { (Poly)Butylmethacrylat } \\ \text { PE } & \text { Polyethylen } \\ \text { PEG } & \text { Polyethylenglycol } \\ \text { PE-N(H }{ }^{+} \text {) } & \text { Polyethylen mit einer (protonierten) Amin } \\ \text { PE-N }{ }^{+} & \text {Polyethylen mit einer quartären Ammoniumendgruppe } \\ \text { PE-N }{ }^{+} & \text {Polyethylen mit einer quartären Ammoniumendgruppe und längerem Spacer } \\ \text { PFQNM } & \text { PeakForce-Quantitative Nanomechanical Mapping }{ }^{\text {TM }} \\ \text { (P)LMA } & \text { (Poly)Laurylmethacrylat } \\ \text { (P)MA } & \text { (Poly)Methylacrylat } \\ \text { (P)MMA } & \text { (Poly)Methylmethacrylat } \\ \text { PMDETA } & \text { N,N,N',N",N"-Pentamethyldiethylentriamin } \\ \text { PP } & \text { Polypropylen } \\ \text { PS } & \text { Polystyrol } \\ \text { PTK } & \text { Phasentransferkatalysator } \\ \text { RAFT } & \text { reversible addition-fragmentation chain transfer } \\ \text { RAFT-COOH } & \text { 2-(Dodecylthiocarbonothioylthio)propionsäure } \\ \text { RAFT-Amin } & \text { 3-(Dimethylamino)propyl-2-(Dodecylthiocarbonothioylthio)propansäureester } \\ \text { RAFT-N }{ }^{+} & \text {RAFT-Agens mit einer quatären Ammoniumgruppe } \\ \text { RAFT-N }{ }^{2+} & \text { RAFT-Agens mit zwei quatären Ammoniumgruppen } \\ \text { rt } & \text { Raumtemperatur } \\ \text { S/R } & \text { Signal-zu-Rauschverhältnis } \\ \text { SSR } & \text { strong segregation region } \\ \text { TBAB } & \text { Tetrabutylammoniumbromid } \\ \text { TBAI } & \text { Tetrabutylammoniumiodid } \\ \text { THF } & \text { Tetrahydrofuran } \\ \text { TOAB } & \text { Tetraoctylammoniumbromid } \\ \text { TOF } & \text { time of flight / Flugzeit } \\ \text { TPPB } & \text { Tetraphenylphosphoniumbromid } \\ \text { UV/VIS } & \text { elektromagnetische Strahlung im sichtbaren (VIS) und ultra-violetten (UV) Bereich } \\ \text { WSR } & \text { weak segregation region } \\ \text { z.B. } & \text { zum Beispiel } \\ & \\ & \end{array}$




\section{Symbolverzeichnis}

In dieser Arbeit werden folgende Symbole verwendet:

\begin{tabular}{|c|c|}
\hline$A_{0}$ & \\
\hline$B$ & Biegemodul \\
\hline$B^{\prime}$ & Speichermodul \\
\hline$B^{\prime \prime}$ & Verlustmodul \\
\hline$c$ & Konzentration \\
\hline$\delta$ & chemische Verschiebung \\
\hline$\bigoplus$ & Dispersität $=\frac{M_{\mathrm{w}}}{M_{\mathrm{n}}}$ \\
\hline E & Elastizitätsmodul \\
\hline$\vec{E}$ & elektrisches Feld \\
\hline$\epsilon_{\mathrm{AA}}$ & Wechselwirkungsenergie zwischen Monomer $A$ und Monomer $A$ \\
\hline$\epsilon_{\mathrm{AB}}$ & Wechselwirkungsenergie zwischen Monomer $A$ und Monomer B \\
\hline$\epsilon_{\mathrm{B}}$ & Bruchdehnung \\
\hline$\epsilon_{\mathrm{BB}}$ & Wechselwirkungsenergie zwischen Monomer B und Monomer B \\
\hline$f_{\mathrm{A}}$ & Volumenbruch der Komponente A \\
\hline$f_{\mathrm{B}}$ & Volumenbruch der Komponente B \\
\hline$F$ & Kraft \\
\hline$h$ & Plancksches Wirkungsquantum \\
\hline$k_{\mathrm{B}}$ & Boltzmannkonstante \\
\hline$\lambda$ & Wellenlänge \\
\hline$l$ & Länge \\
\hline$l_{0}$ & ursprüngliche Länge \\
\hline$M_{\mathrm{n}}$ & Zahlenmittel der Molmasse \\
\hline$M_{\mathrm{p}}$ & Masse am Peakmaximum \\
\hline$M_{\mathrm{w}}$ & Gewichtsmittel der Molmasse \\
\hline$m / z$ & Masse-zu-Ladungsverhältnis \\
\hline$\nu$ & Frequenz \\
\hline$n / N$ & Polymerisationsgrad \\
\hline$R$ & Massenauflösung \\
\hline$R_{\mathrm{e}}$ & Streckgrenze \\
\hline$R_{\mathrm{m}}$ & Zugfestigkeit \\
\hline$\sigma$ & nominelle Zugspannung \\
\hline$t$ & Zeit \\
\hline$T$ & Temperatur \\
\hline$T_{\mathrm{g}} \chi$ & Flory-Huggins-Parameter \\
\hline$U$ & Umsatz \\
\hline$y$ & Koordinationszahl \\
\hline$z$ & Ladung \\
\hline$Z$ & Zähigkeit \\
\hline
\end{tabular}




\section{Einheitenverzeichnis}

$\begin{array}{ll}\% & \text { Prozent } \\ { }^{\circ} \mathrm{C} & \text { Grad Celsius } \\ \text { bar } & \text { Bar } \\ \mathrm{d} & \text { Tag } \\ \mathrm{g} & \text { Gramm } \\ \mathrm{h} & \text { Stunde } \\ \mathrm{m} & \text { Meter } \\ \mathrm{mol} & \text { Mol } \\ \mathrm{mol} \% & \text { Molprozent } \\ \text { ppm } & \text { parts per million } \\ \text { wt\% } & \text { Gewichtsprozent }\end{array}$





\section{Einleitung}

Die Entdeckung neuer Materialien war für die Menschheit schon immer von großer Bedeutung, weshalb mit der Stein-, Bronze- und Eisenzeit ganze Epochen der Menschheitsgeschichte nach Werkstoffen benannt wurden. Die aus organischen Makromolekülen bestehenden Kunststoffe gehören in der heutigen Zeit mit einer Produktionsmenge von etwa 300 Millionen Tonnen Kunststoffe pro Jahr zu den wichtigsten Werkstoffen. Sie sind in unserem Alltag von einfachen Plastiktüten bis hin zu Hochleistungsmaterialien wie kugelsicheren Westen und Flugzeugtrageflächen aus Kevlar allgegenwärtig. 11 Große Vorteile der Polymere gegenüber vielen andere Materialien sind ihre oftmals geringen wirtschaftlichen Herstellungskosten, ihr geringes Gewicht, ihre guten mechanischen Eigenschaften und ihre hohe Korrosionsbeständigkeit. Zudem lassen sich die Eigenschaften der Kunststoffe beispielsweise durch Additive, Polymermischungen, Comonomere und Blockcopolymerisation über weite Bereiche gezielt anpassen. Zudem lassen sich Thermoplasten mittels thermischer Behandlung und verschiedener Verfahren umformen und verarbeiten. Sie können daher auch leicht recycelt werden. 112

Mit der Entwicklung der kontrollierten radikalischen Polymerisationen in den 80er und 90 er Jahren des letzten Jahrhunderts stehen neue und robuste Techniken zur Herstellung von maßgeschneiderten Blockcopolymeren und anderen Polymerarchitekturen bereit. $3 / 4$ In Blockcopolymeren weist die Polymerkette mindestens zwei Blöcke aus verschiedenen Monomeren $A$ und $B$ auf, die kovalent untereinander verknüpft sind. Blockcopolymere kombinieren daher die Eigenschaften beider Homopolymere A und B. Durch die gezielte Wahl eines weichen und harten Blocks ist es zudem möglich neuartige Materialien wie thermoplastische Elastomere herzustellen. ${ }^{55-7]}$ Darüber hinaus sind Blockcopolymere aufgrund von Mikrophasenseparation dazu in der Lage sich in der Schmelze selbst ordnende Nanostrukturen zu erzeugen. Diese finden ebenso wie die in selektiven Lösungsmitteln entstehenden mizellaren Strukturen vielfältige Anwendungen in der Industrie und Forschung. 8

Auch durch die Vernetzung einzelner Makromoleküle zu einem dreidimensionalen Netzwerk können die mechanischen Eigenschaften in Form des E-Moduls und der Härte sowie die thermische und chemische Stabilität der Kunststoffe erhöht werden. 9110] Das Umformen und das Recycling des vernetzten Werkstoffes ist dann jedoch nicht mehr möglich. Eine reversible Vernetzung wäre dementsprechend ideal, um vernetzte Kunststoffe nach Gebrauch wiederverwenden zu können und so die Belastung der Umwelt mit Kunststoffabfall zu reduzieren.

Ein Ziel dieser Arbeit ist es daher die reversible Photovernetzung auf Basis der Cycloadditionen auf ihren Einsatz zur Herstellung vernetzter und recyclebarer Werkstoffe hin zu überprüfen. Dabei soll der Einfluss der gezielten reversiblen Vernetzung der weichen oder harten Phase eines mikrophasenseparierten thermoplastischen Blockcopolymers auf die mechanischen Eigenschaften des Polymers untersucht werden. 
Darüber hinaus hängen viele Eigenschaften der Kunststoffe von ihrer Molmassenverteilung ab. 11-13 Folglich ist es auch in der Industrie und in der Qualitätssicherung von großem Interesse die Molmasse gezielt einzustellen und überprüfen zu können. Zur Bestimmung der Molmasse und der Molmassenverteilung wird in der Regel die Gelpermeationschromatographie (GPC) in Kombination mit einem geeigneten Detektor verwendet. Sie ist jedoch eine relative Methode und benötigt daher für die korrekte Molmassenbestimmung die MarkHouwink-Parameter oder Kalibrierstandards des gleichen Polymers. 14]

Seit der Entwicklung der weichen lonisationsmethoden matrix-assisted laser desorption ionization (MALDI) und Elektrospray-Ionisation (ESI), die mit der Verleihung des Nobelpreises im Jahr 2002 gewürdigt wurde, ist es auch möglich Polymere mittels Massenspektrometrie (MS) zu analysieren. 15-17] Mit der MS kann auch ohne Kenntnis der Mark-HouwinkParameter die absolute Molmasse von Polymeren bestimmt werden. [18[19] Die Massenspektrometrie hat zudem den Vorteil, dass aufgrund der hohen Massenauflösung einzelne Polymerketten voneinander unterschieden und sogar deren Endgruppen und molekulare $\mathrm{Zu}$ sammensetzungen mit nur einer Analysemethode bestimmt werden können. 20|21] Die lonisierung der Polymerketten erfolgt aufgrund fehlender basischer Seitengruppen in der Regel durch die Adduktbildung mit Metallionen. In der ESI-MS treten mit zunehmender Kettenlänge mehrfach geladene Ketten durch Anlagerung mehrerer Kationen auf. Da Polymere selbst eine Molmassenverteilung aufweisen entstehen durch die zusätzliche Ladungsverteilung in der ESI-MS schnell komplexe und schwer interpretierbare Massenspektren. [18[19] Die MALDI-MS ist zwar eine sensitive Methode, die Massenspektren weisen jedoch oft nur ein geringes Signal-zu-Rausch-Verhältnis auf.

Unfunktionalisierte Polyolefine können massenspektrometrisch nicht analysiert werden, da sie keine polaren Bindungsstellen zur Anlagerung eines Kations oder Anions besitzen. Ein Lösung ist hier der Einbau von polaren (End)gruppen. [22-24] Klassische Polymerisationstechniken wie die radikalische Polymerisation, das Ziegler-Natta-Verfahren oder auch die Verwendung von homogenen Metallocenkatalysatoren bieten jedoch keine oder nur stark eingeschränkte Möglichkeiten der Endgruppenmodifizierung. Die catalyzed chain growth (CCG) Polymerisation verwendet eine Alkylmetallverbindung als Kettentransferreagenz, um kontrollierte Polyolefine herzustellen und die Endgruppe mittels metallorganischer Reaktionen zu modifizieren. 25126]

Ein weiteres Ziel dieser Arbeit ist es daher mittels kontrollierter radikalischer Polymerisation Polymere mit einer kationischen Endgruppe herzustellen und diese mittels ESI- und MALDI-MS zu untersuchen. Es wird vermutet, dass durch die Verwendung der ionischen Endgruppe die Sensitivität und das Signal-zu-Rausch-Verhältnis wesentlich verbessert sowie die Anzahl der Ladungen pro Kette in der ESI-MS reduziert und daher das Massenspektrum vereinfacht wird. Zudem soll anhand von Polyethylen gezeigt werden, dass eine kationische Endgruppe die massenspektrometrische Analyse von Polyolefinen ermöglicht und dass sich durch diese Endgruppe weitere Anwendungsfelder für Polyethylen ergeben. 


\section{Theorie und Kenntnisstand}

\subsection{Kontrollierte reversibel-desaktivierende radikalische Polymerisationstechniken}

In den letzten Jahren wurden mehrere kontrollierte reversibel-desaktivierende radikalische Polymerisationstechniken (KRP) entwickelt, die die Vorteile der freien radikalischen Polymerisation (FRP) mit der der lebenden Polymerisation verbinden. Sie ermöglichen also die Synthese von Polymeren mit kontrollierbarer Molmasse und enger Molmassenverteilung sowie die Synthese komplexer Polymerarchitekturen wie z.B. Block- oder Sternpolymere. 3/4 Die bekanntesten Vertreter sind die Nitroxid-vermittelte Polymerisation (nitroxide-mediated polymerization, NMP) 2728, die Atom-Transfer radikalische Polymerisation (ATRP) ${ }^{429]}$ und die Reversible Additions-Fragmentierungs Kettenübertragungs (reversible addition-fragmentation chain transfer, RAFT)-Polymerisation. ${ }^{330]}$ Allen KRP gemeinsam ist, dass zu gegebener Zeit nur ein kleiner Teil der Ketten aktiv ist und Monomere addieren kann. Die Mehrheit der wachsenden Ketten befindet sich in einem reversibel deaktivierten Zustand. Die aktive und deaktivierte Spezies stehen dabei im Gleichgewicht und die Molmasse steigt mit dem Monomerverbrauch. $3 / 429$

In der ATRP wird ein ligandstabilisiertes Übergangsmetallhalogenid - zu meist $\mathrm{Cu}(\mathrm{I}) X \mathrm{~L}_{n}$ - und ein Alkylhalogenid R'-X als Initiator verwendet. Im Initiierungsschritt reagieren das $\mathrm{Cu}(\mathrm{I}) X \mathrm{~L}_{n}$ und der $\mathrm{R}^{\prime}-\mathrm{X}$ im Gleichgewicht zu einem kleinen Teil zu Cu(II) $\mathrm{X}_{2} \mathrm{~L}_{n}$ und dem Initiatorradikal R'. (Schema 2.1). ${ }^{429]}$ Das R'. initiiert die Polymerisation durch Monomeraddition.

Initiierung:

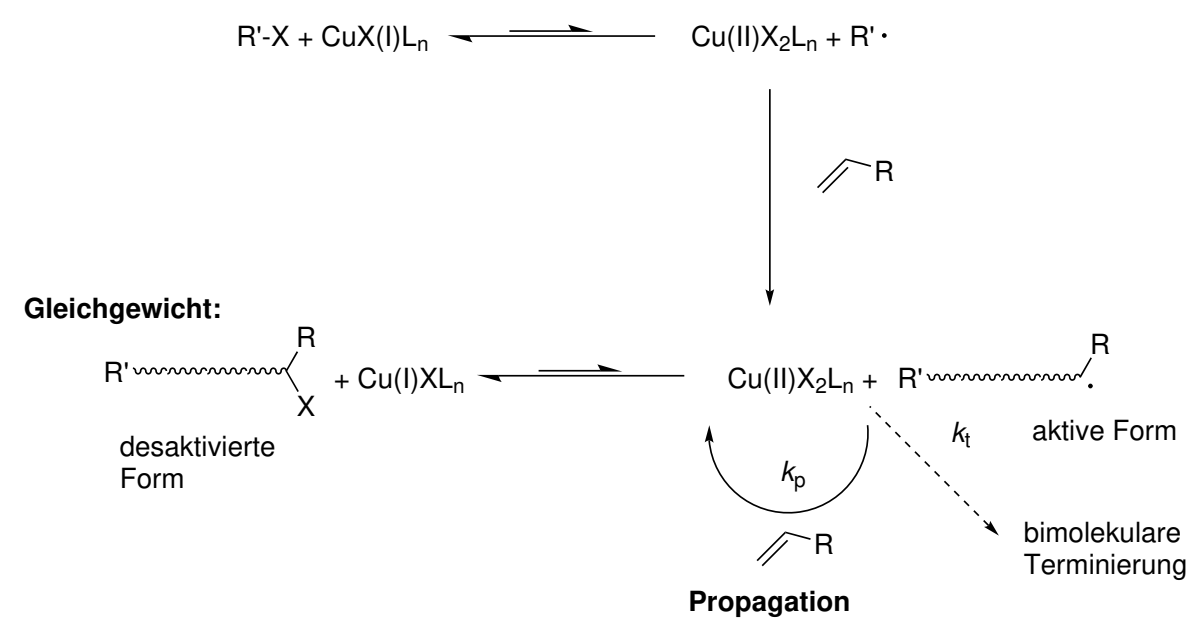

Schema 2.1. Der Mechanismus der ATRP (angelehnt an die Literatur). 4 429 
A

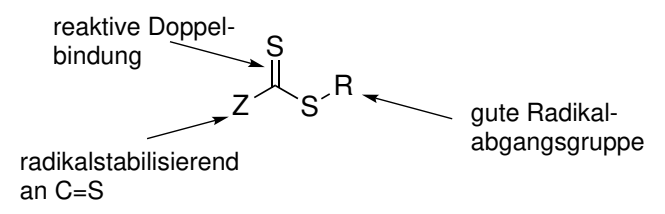

B

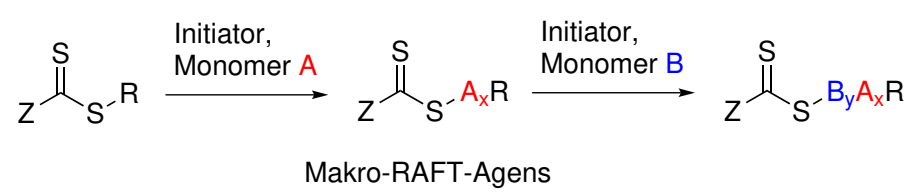

Abb. 2.1. Die allgemeine Struktur eines RAFT-Agens $(A)$ und die schematische Synthese eines Blockcopolymers (B).

Das entstandene polymere Radikal entspricht der aktiven Form und wird durch die $\mathrm{Cu}$ (II)Spezies wieder zur deaktivierten Alkylverbindung oxidiert. Da der Austausch von aktiver und deaktivierter Form wesentlich schneller ist als die Monomeraddition, wachsen im Mittel alle Ketten gleich schnell. Am Ende der Polymerisation befinden sich die meisten Ketten im deaktivierten Zustand und können daher z.B. für eine Blockcopolymerisation verwendet werden.

Im Falle der RAFT-Polymerisation wird neben Monomer, Initiator und gegebenenfalls Lösungsmittel ein sogenanntes RAFT-Agens im Überschuss bezogen auf den Initiator hinzugegeben. Dieses RAFT-Agens dient als äußerst effektives Kettentransferreagenz. Die allgemeine Struktur eines RAFT-Agens basierend auf einer Thiocarbonylthio-Verbindung ist in Abb. 2.1A gezeigt. Es enthält eine reaktive $\mathrm{C}=\mathrm{S}$-Doppelbindung, eine Z-Gruppe (z.B. Alkohole, Alkanthiol oder Phenylrest), welche radikalstabilisierend am Kohlenstoff der C=SDoppelbindung ist, und eine R-Gruppe, die eine gute radikalische Abgangsgruppe ist. Die Wahl der Z- und R-Gruppe ist dabei abhängig von der Reaktivität des Monomers. In dieser Arbeit werden für die Synthese der Poly[(Meth-)Acrylate] und Polystyrole Trithiocarbonate als RAFT-Agenzien verwendet, da sie stabiler sind und weniger Retardierung zeigen als Dithiobenzoate. 3132] Der Mechanismus einer RAFT-Polymerisation ist in Schema 2.2 gezeigt. ${ }^{3]}$ Ein durch Zerfall des Initiators entstandenes Initiatorradikal wird durch Addition mehrerer Monomer in eine polymere radikalische Spezies $P_{n}$. umgewandelt, die dann an die $\mathrm{C}=\mathrm{S}$-Doppelbindung des RAFT-Agens addieren kann (auch eine direkte Addition des Initiatorradikals an das RAFT-Agens ist möglich). Das dabei entstehende Intermediatradikal fragmentiert im Vorgleichgewicht, sodass entweder erneut $P_{n}$. oder die R-Gruppe als Radikal $R$. frei wird. Das $R$. reinitiiert dann die Polymerisation, indem es durch Monomeraddition zu $P_{m}$. propagiert. Im Hauptgleichgewicht befinden sich die aktiven Polymerketten $P_{n}$. im 
1. Initiierung

$$
\mathrm{I}_{2} \stackrel{\Delta \mathrm{T}}{\longrightarrow} \mathrm{I} \stackrel{\mathrm{M}}{\longrightarrow} \mathrm{P}_{\mathrm{n}}
$$

2. Vorgleichgewicht

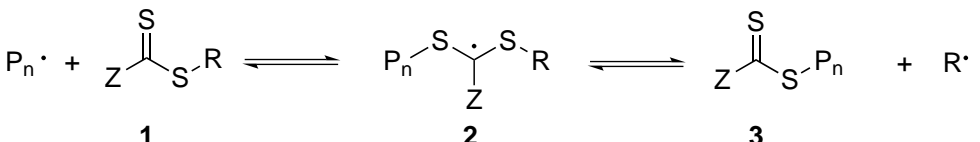

$$
\begin{aligned}
& \text { 3. Reinitiierung } \\
& R \cdot \stackrel{M}{\longrightarrow} P_{m} \text {. }
\end{aligned}
$$

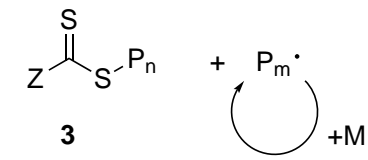

5. Terminierung
$\mathrm{P}_{\mathrm{n}} \cdot+\mathrm{P}_{\mathrm{m}} \cdot \longrightarrow$ Rekombination oder Disproportionierung

Schema 2.2. Der Mechanismus der RAFT-Polymerisation (angelehnt an die Literatur). ${ }^{3}$

Gleichgewicht mit der reversibel deaktivierten Spezies 3. Fragmentierung und Addition sind dabei wesentlich schneller als die Propagation. Durch den schnellen Austausch der aktiven (Radikale) und reversibel deaktivierten Spezies wachsen im Mittel alle Ketten gleich schnell. Wie Abb. 2.1B zeigt, werden die Monomere A zwischen RAFT- und R-Gruppe insertiert. Das entstandene Polymer ist somit selbst ein sogenanntes Makro-RAFT-Agens und kann in einem weiteren Schritt mit einem Monomer B zu einem Blockcopolymer umgesetzt werden.

Die RAFT-Polymerisation kann für eine Vielzahl von Monomeren angewendet werden. ${ }^{330]}$ Sie toleriert viele funktionelle Gruppen und Lösungsmittel. Im Gegensatz zu ATRP wird auch kein Schwermetall benötigt, dessen Abtrennung vom Polymer aufwendig ist. Bekannte Synthesevorschriften von Polymeren aus der FRP können in der Regel ohne großen Aufwand übernommen werden, da sich die RAFT-Polymerisation nur durch die Zugabe des RAFT-Agens unterscheidet.

\subsection{Kontrollierte Polymerisation von Polyethylen}

Traditionelle Synthesen von PE über einem freien radikalischen Prozess (low density Polyethylen) oder über das Ziegler-Natta-Verfahren produzieren PE mit einer breiten Molmassenverteilung. ${ }^{33]}$ Die Endgruppenmodifikation von PE ist sowohl mit den zuvor genannten Verfahren, als auch mit der homogenen PE-Polymerisation mittels Metallocenkatalysatoren noch immer schwierig. 


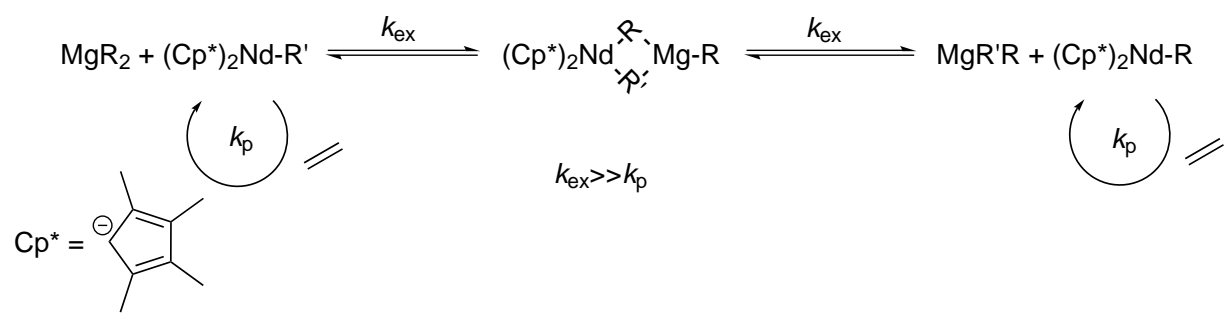

Schema 2.3. Der Mechanismus der CCG Polymerisation (angelehnt an die Literatur). 34

Die Entwicklung der catalyzed chain growth (CCG) Polymerization ermöglicht die Synthese von endgruppenfunktionalisiertem PE mit einer engen Molmassenverteilung (MMV). Der Mechanismus der CCG Polymerisation ist anhand des in dieser Arbeit verwendeten Katalysators im Schema 2.3 gezeigt. ${ }^{34}$ Die Dialkylmagnesiumverbindung dient als Aktivator für den Präkatalysator $\left(\mathrm{Cp}^{*}\right)_{2} \mathrm{NdCl}_{2}\left(\mathrm{Cp}^{*}=\right.$ Tetramethylcyclopentadienylanion $)$ und gleichzeitig als Kettentransferreagenz. Analog zu den KRPs ist auch hier der Austausch der Ketten schneller als die Propagation, sodass Polymere mit einer schmalen MMV erhalten werden. Das PE liegt ebenfalls als Dipolyethylenmagnesium PE-Mg-PE vor. Dadurch kann die Endgruppe des PEs mittels typischer metallorganischer Reaktionen modifiziert werden, was ein großer Vorteil der CCG Polymerisation ist.

Die Einführung verschiedener Endgruppen wie lod, Azid, Hydroxyl, Epoxid, Thiol oder Amin wurde bereits in der Literatur beschrieben. 24/34/35] Des Weiteren wurde die Möglichkeit der Endgruppenfunktionalisierung genutzt, um PE mit ATRP-, NMP- und RAFT-Gruppen oder -Initiatoren zu synthetisieren. Dadurch ist die Herstellung von PEBlockcopolymeren möglich. $34[36$ Schema 2.4 zeigt beispielhaft die lod-, Azid- und AminEndgruppenfunktionalisierung von PE.

$$
\text { PE-Mg-PE } \underset{-\mathrm{Mgl}_{2}}{\stackrel{\mathrm{I}_{2}}{\longrightarrow}} 2 \mathrm{PE}-\mathrm{I} \stackrel{-\mathrm{N}_{3}}{\longrightarrow} 2 \text { PE-N } \frac{\text { Reduktion }}{\longrightarrow} 2 \mathrm{PE}-\mathrm{NH}_{2}
$$

Schema 2.4. Die lod-, Azid- und Amin-Endgruppenfunktionalisierung von mittels CCG Polymerisation hergestelltem PE. 


\subsection{Massenspektrometrie}

Die Massenspektrometrie (MS) ist eine der wichtigsten und sensitivsten Analysetechniken in der qualitativen und quantitativen Analytik. Sie erlaubt es Analytmoleküle anhand ihrer Masse zu identifizieren. Fragmentierung des Analyten innerhalb des Massenspektrometers ermöglicht zudem einen Rückschluss auf dessen Struktur. Abb.2.2 zeigt den schematischen Aufbau eines Massenspektrometers. ${ }^{37-39]}$ Nach dem Probeneinlass wird der Analyt ionisiert und in die Gasphase überführt. Dies geschieht zumeist im Vakuum. Nicht ionisierte Moleküle werden zum Großteil durch Vakuumpumpen abgepumpt während ionisierte Moleküle durch magnetische und bzw. oder elektrische Linsen zum Massenanalysator transportiert werden. Im Massenanalysator erfolgt eine Auftrennung der Analyten entsprechend ihres Masse-zuLadungsverhältnisses $m / z$, welches per Definition in der Massenspektrometrie ohne Einheit ist. Die lonen werden dann am Detektor, oftmals Sekundärelektronenvervielfachen, detektiert. Die Daten werden dann mittels Computer erfasst und verarbeitet.

Zur Auftrennung der Ionen werden Quadrupol-40], Ionenfallen- (linear ${ }^{[41]}$, Quadrupol [2[43], Orbitrap 44[45] und Fouriertransformations-lonenzyklotronresonanz ${ }^{\text {46[47]), }}$ Sektorfeld-48] und Flugzeitmassenspektrometer (time of flight, TOF) verwendet. 49-52] Letzteres wird auch in dieser Arbeit verwendet. In der TOF-MS werden die lonen durch ein elektrisches Feld beschleunigt (Abb. 2.3). 39/49-52] Während der feldfreien Flugstrecke teilen sich die lonen anhand ihres $m / z$-Verhältnisses auf, da leichtere lonen auf höhere Geschwindigkeiten beschleunigt werden. Die Flugzeit $t$ ist dabei proportional zu $\sqrt{m / z}$. Der Reflektron verlängert dabei zum einen platzsparend die Flugstrecke, zum anderen wird die Energieverteilung erniedrigt und so die Massenauflösung erhöht. Die Massenauflösung $R$ entspricht dem kleinsten Massenunterschied $\Delta m$ der von einem Peak mit gegebener Masse $m$ noch getrennt werden kann.

$$
R=\frac{m}{\Delta m}
$$

Viele TOF-Massenspektrometer besitzen zwei Detektoren. Im linearen Modus wird der Reflektron nicht verwendet und die lonen werden mittels des linearen Detektors detektiert. Im linearen Modus wird die Sensitivität erhöht und die Fragmentierung verringert, aber die Massenauflösung erniedrigt. Neben der klassischen Elektronenstoßionisation, die zu Fragmentierung des Analytions führt, wurden mehrere sogenannte „weiche“ Ionisationsmethoden

Vakuum

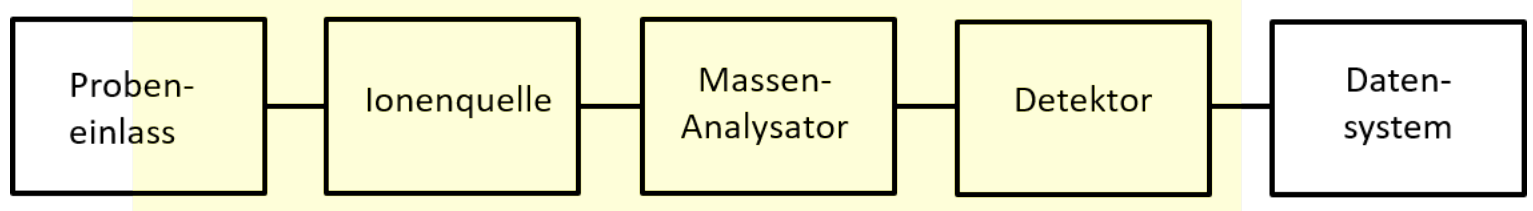

Abb. 2.2. Schematischer Aufbau eines Massenspektrometers. 


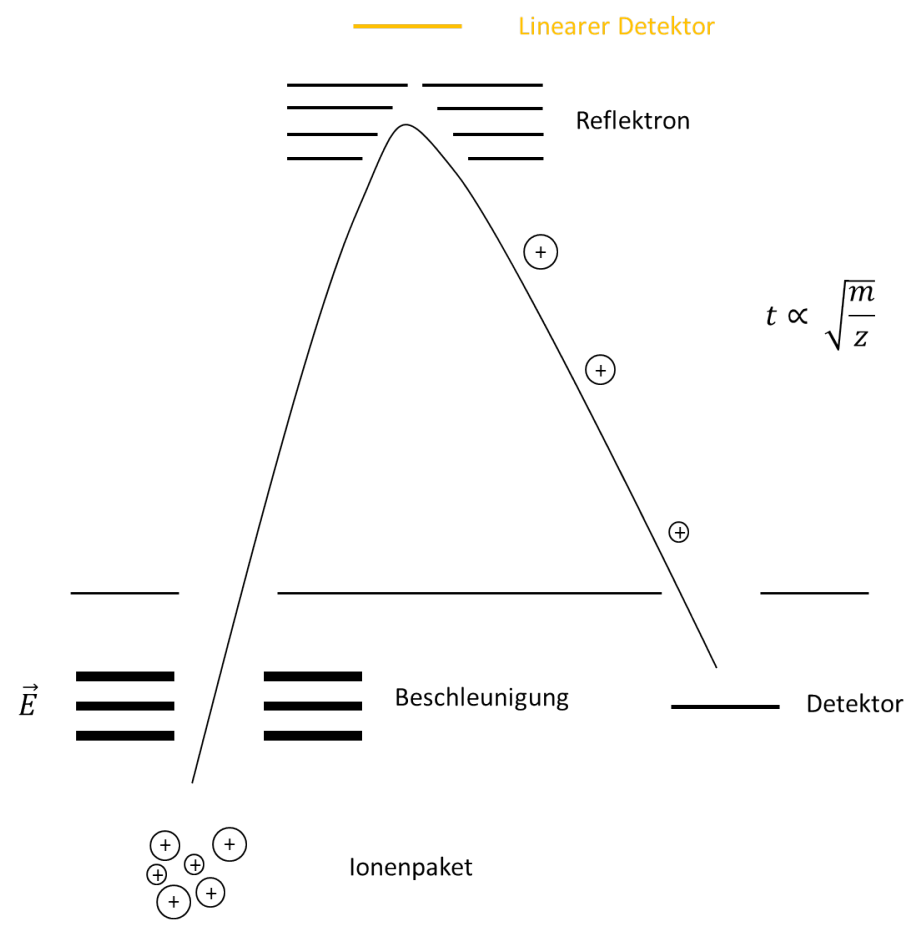

Abb. 2.3. Aufbau eines Flugzeitmassenspektrometers, das im linearen oder in Reflektronmodus betrieben werden kann.

entwickelt, die zu keiner oder zu nur wenig Fragmentierung führen. 39153-55] Die ElektrosprayIonisation (ESI) und die Matrix-unterstützte Laser-Desorption-Ionisation (matrix-assisted laser desorption ionization, MALDI) sind heute in der Analytik von synthetischen und biologischen Polymeren als weiche lonisationmethoden etabliert. ${ }^{[15-17756 / 57]}$ Diese beide Methoden werden auch in dieser Arbeit in Kombination mit einem TOF-Massenanalysator verwendet. Im Folgenden wird daher auf diese Techniken näher eingegangen.

\subsubsection{ESI-MS}

Entgegen Abb.2.2ffindet bei der ESI-MS die Ionisation außerhalb des Massenspektrometers und bei Atmosphärendruck statt. Dazu wird der Analyt gelöst in einem polaren und flüchtigen Lösungsmittel durch eine Kapillare gegeben, an der eine hohe Spannung (2-5 kV) anliegt (Abb. 2.4). 1513839158 Im Falle einer positiven Spannung an der Kapillare werden Anionen zum Teil neutralisiert, weshalb sich ein Überschuss an positiver Ladungen in der Lösung aufbaut. Aufgrund des Wechselspiels aus der Oberflächenspannung der Flüssigkeit und der sich abstoßenden elektrischen Kraft zwischen den positiven Ladungen bildet sich am Ende der Kapillare ein sogenannter Taylor-Konus. Aus diesem Kegel wird ein Faden emittiert der in einen Sprühnebel zerfällt. Die entstandenen geladenen Tröpfchen werden dann, unterstützt durch einen heißen Gasgegenstrom, durch Verdampfung des Lösungsmittels immer kleiner bis das Rayleigh-Limit erreicht ist. An diesem Punkt ist die Abstoßung der lonen unterein- 


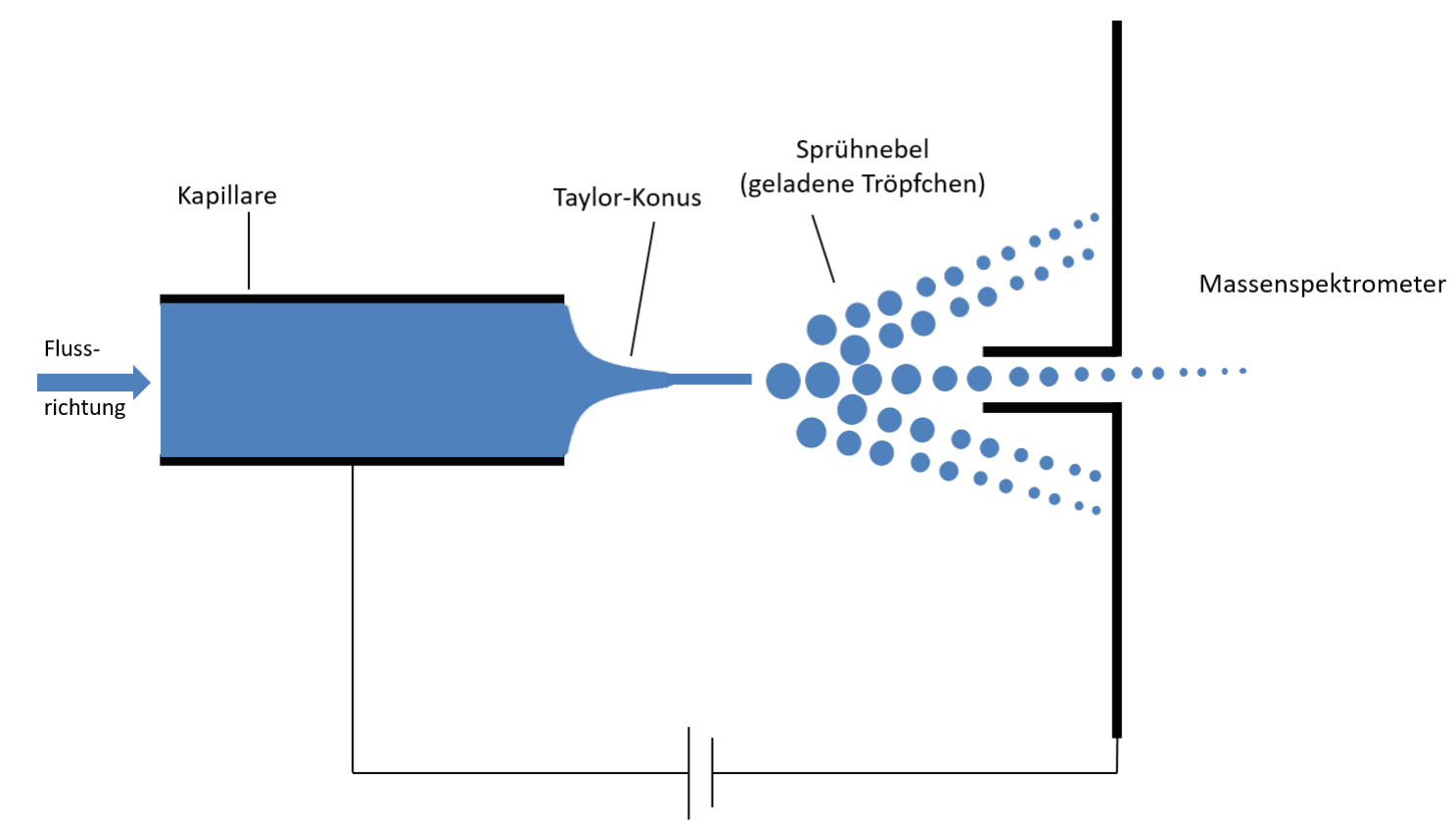

Abb. 2.4. Darstellung des Elektrospray-lonisationsprozesses.

ander größer als die Oberflächenspannung. Es findet eine Coulombexplosion statt und der große Tropfen zerfällt in mehrere kleinere Tropfen (Abb. 2.5). Letztlich kommt es zur Bildung von unsolvatisierten Analytionen, die aufgrund der Potentialdifferenz zwischen Kapillare und dem Eingang des Massenspektrometers ins Massenspektrometer gelenkt werden. Es gibt mehrere theoretische Modelle, die die Entstehung der unsolvatisierten Analytionen erklären. $55[58,61]$

Beim charged residue Modell (CRM; Abb. 2.5) wiederholt sich die Coulombexplosion bis nur noch ein Analytion von einer dann verdampfenden Solvathülle umgeben ist. Es wird vor allem für die Erklärung der Desolvatisierung großer Moleküle wie polarer Proteine in ähnlich polaren Lösungsmitteln verwendet. Nach dem ion evaporation Modell (IEM) schrumpfen die Tropfen durch Verdampfung des Lösungsmittels bis die Feldstärke an der Oberfläche des Tropfens so hoch ist, dass einzelne solvatisierte Analytionen emittiert werden können (Abb. 2.5). Die Solvathülle der lonen verdampft dann entweder im Gasgegenstrom oder im Vakuum der Massenspektrometers. Das IEM ist vor allem für kleine Analytmoleküle, besonders wenn sie oberflächenaktiv sind, und bei Tröpfchengrößen von unter $10 \mathrm{~nm}$ gültig. Ein neueres Modell ist das chain ejection Modell (CEM), welches speziell für unpolare synthetische und Biopolymere entwickelt wurde und im Prinzip eine Erweiterung des IEMs ist. 60 -63 Aufgrund ihres unpolaren Charakters migriert die Polymerkette zur Tropfenoberfläche und wird dort durch Kationen positiv geladen (Abb. 2.6). Ein Ende der Kette kann dann samt Ladungen den Tropfen verlassen und es folgt eine stufenweise Ausstoßung der vollständigen Kette.

Die ESI-MS kann sowohl im positiven als auch im negativen Modus betrieben werden. Analytionen weisen dann die gleiche Polarität der Ladung auf und werden beim Vorhanden- 


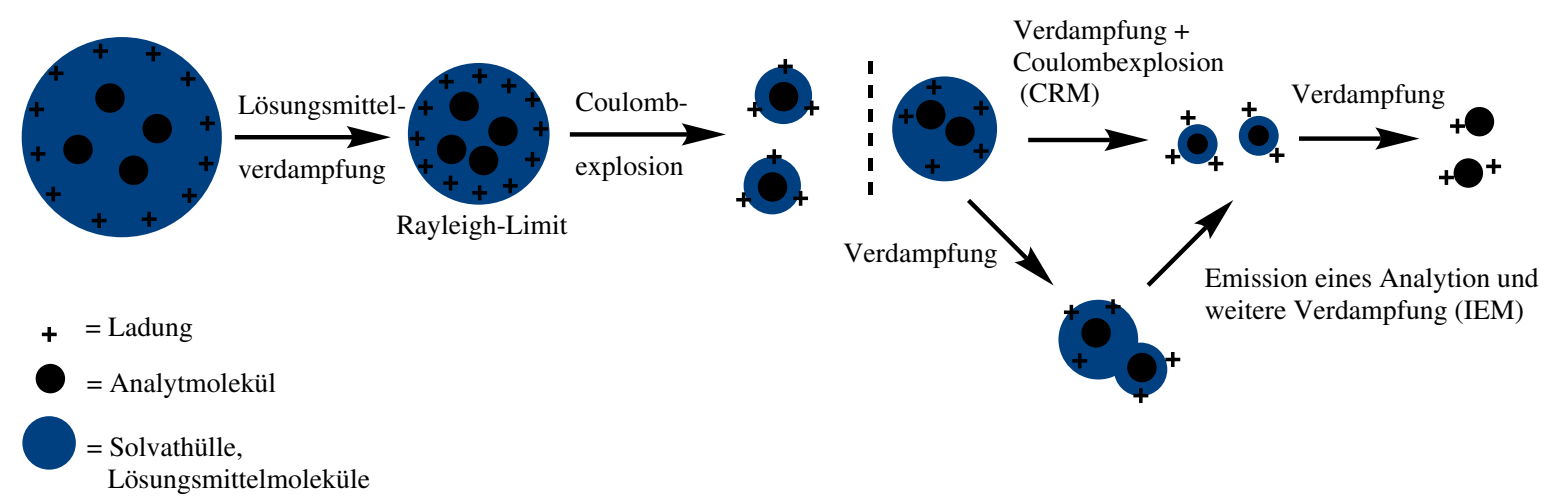

Abb. 2.5. Darstellung der Coulombexplosion, des CRMs und des IEMs.

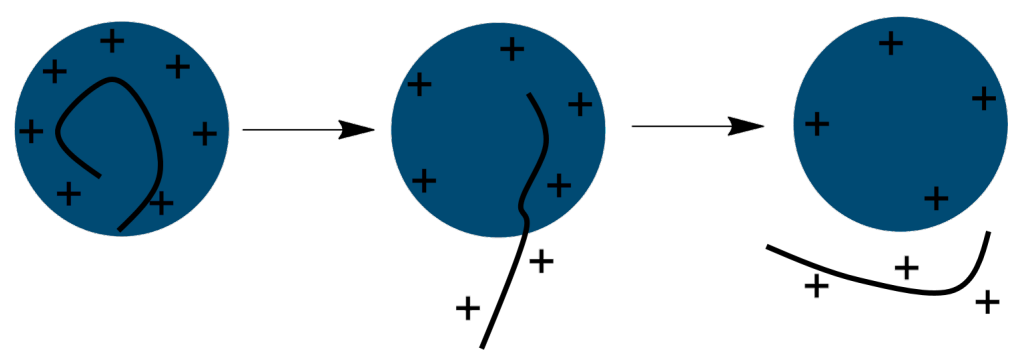

Abb. 2.6. Schematischen Darstellung der stufenweise Ausstoßung der geladenen Polymerketten (schwarz) aus dem Lösungsmitteltropfen nach dem CEM.

sein von basischen oder sauren Gruppen zu meist als Quasimolekülionen - sprich $\left[\mathrm{M}_{+} \mathrm{H}^{+}\right]$

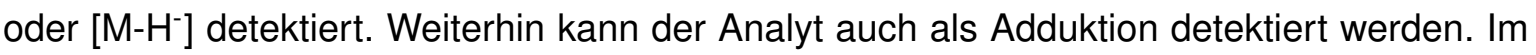
positiven Modus bilden sich Adduktionen des Analyten mit Metallkationen (z.B. $\left[\mathrm{M}+\mathrm{Na}^{+}\right]$in sauerstoffreichen Analyten). Die ESI führt bei höher molekularen Verbindungen wie Proteinen und synthetischen Polymeren oft zu einer Ladungsverteilung mit mehreren Ladungen. $15[3964$

\subsubsection{MALDI-MS}

In der MALDI-MS wird das Polymer nicht in Lösung ionisiert, sondern in eine feste Matrix - meist organisch-kristalline Verbindungen mit einem aromatischen $\pi$-System - eingebettet. ${ }^{39655-67]}$ Dazu wird eine verdünnte Lösung des Analyten mit einer wesentlich konzentrierteren Lösung der Matrix gemischt. Des Weiteren wird zur lonisation entweder im positiven Modus eine Säure (im positiven Modus), eine Base (im negativen Modus) oder ein Salz (Metallkationenaddukte im positiven Modus) zugegeben, da der Analyt analog zur ESI-MS als Quasimolekülion oder Adduktion detektiert wird. Oftmals enthält aber bereits die Matrix eine saure Gruppe, die ausreichend zur Protonierung des Analyten ist. Ein Tropfen (0,5-2 $\mu \mathrm{L})$ der gemischten Lösung wird dann auf eine MALDI-Platte gegeben. Während der Verdampfung des Lösungsmittels kristallisiert die Matrix und schließt dabei den verdünnten Analyten ein. Nach dem Einlegen der Platte in das MALDI-Spektrometer, wird das Gerät auf etwa 


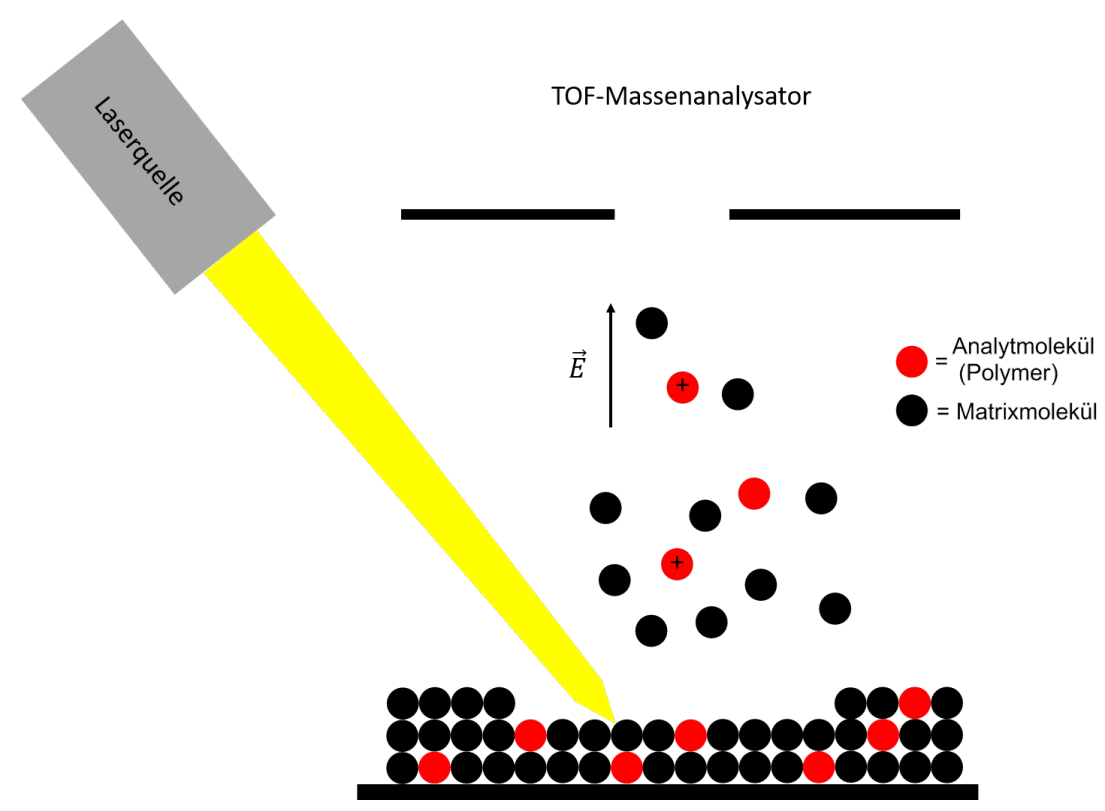

Abb. 2.7. Schematische Darstellung des MALDI-Experiments.

$10^{-6}$ mbar evakuiert. Die Probe wird dann in der Kammer mit einem UV-Laser (meist Stickstofflaser oder frequenzverdreifachten Nd:YAG-Laser mit $\lambda=337 \mathrm{~nm}$ oder $355 \mathrm{~nm}$ ) bestrahlt (Abb. 2.7). Die Matrixmoleküle absorbieren die UV-Strahlung, erwärmen sich dadurch und gehen in die Gasphase über. Dabei wird der enthaltene und durch Säure bzw. Base oder Metalladdukte ionisierte Analyt ebenfalls mit in die Gasphase getragen. Auch eine Gasphasenionisierung oder Photoionisierung (führt zu $\mathrm{M}^{+\cdot}$-lonen) bei absorbierenden Analyten ist möglich oder wird diskutiert. Letztlich ist der Mechanismus der lonenerzeugung in der MALDI aber noch immer ungeklärt. Die lonen werden dann durch ein elektrisches Feld in den TOF-Massenanalysator beschleunigt. Im über der Oberfläche entstandenen Plasma befinden sich Kat- und Anionen. Die Beschleunigungsspannung bestimmt, welche lonensorte extrahiert wird.

Im Gegensatz zur ESI produziert die MALDI in der Regel einfach geladene lonen. Erst bei sehr hohen Molmassen werden Ketten mit mehr als einer Ladung beobachtet. ${ }^{68}$ Eine mögliche Erklärung liefert das lucky survivor-Modell. ${ }^{3969}$ Der bevorzugte Prozess im Plasma ist die Rekombination von Kat- und Anionen. Dabei ist die Rekombinationsrate im Falle von mehrfach geladenen Spezies wesentlich schneller als bei einfach geladenen lonen. Dies erklärt auch die geringeren Intensitäten von negativen lonen, da die leichten Elektronen schnell kombinieren. Die Probenpräparation hat einen großen Einfluss auf die Spektrenqualität und ist daher ein entscheidender Schritt. 39/70|71] Sowohl die Wahl der Matrix, das Verhältnis von Matrix zu Analyt, als auch der Kristallisationsprozess sind dabei sehr wichtig. Die Bildung von kleinen Kristallen ist für die Spektrenqualität vorteilhaft. 65[66|72-74] Neben der Probenvorbereitung ist die verwendete Laserenergie ein entscheidender Faktor in der MALDI-MS, obwohl durch verzögerte Ionenextraktion die Auflösung der Spektrometer erhöht werden konnte. ${ }^{75}$ Die Laserenergie hat einen Einfluss auf die mittels MALDI-MS de- 
tektierte Molmassenverteilung eines synthetischen Polymers und eine hohe Energie kann zur Fragmentierung der Polymerketten führen. ${ }^{76-78}$ Eine zu hohe Laserenergie führt zudem bei den lonen zu einer breiten Verteilung der kinetischen Energie und daher zu einer starken Verbreiterung der Peaks, sodass die Massenauflösung reduziert wird. ${ }^{[7]}$ Die Massenauflösung ist in der MALDI-TOF-MS im allgemeinen geringer als in der ESI-TOF-MS. Ursache dafür ist die Inhomogenität der Filmdicke des Probenspots, da anfängliche Höhenunterschiede nicht gänzlich ausgeglichen werden können. 3980 Neben der oben erwähnten lösungsmittelbasierten Probenpräparation gibt es auch lösungsmittelfreie Präprationstechniken für unlösliche Analyten. In dem Fall wird die feste Matrix mit dem Analyten vermahlen. 8182

\subsubsection{Massenspektrometrie von Polymeren}

Biopolymere wie Proteine besitzen in der Regel eine genau definierte Molmasse, sie sind monomodal. Synthetische Polymere haben eine Molmassenverteilung (Abb. 2.8 A), da sie aus unterschiedlich langen Ketten einer sich wiederholenden Repetiereinheit (entspricht dem Monomer in der Kettenpolymerisation) bestehen. Das heißt, die Differenz der Molmasse in den Polymerketten entspricht einem Vielfachen der Molmasse der Repetiereinheit. [18] Da die Endgruppen in allen Ketten identisch sein sollten, genügt es für die Endgruppenanalyse in der Regel einen Ausschnitt des Massenspektrums, der eine Monomereinheit umfasst, zu analysieren.

Wie bereits erwähnt, entstehen in der MALDI-MS auch bei höheren Molmassen fast ausschließlich einfach geladene Polymerketten (Abb. 2.8A), wohingegen in der ESI-MS bereits bei geringen Molmassen einfach und mehrfach geladene Ketten erhalten werden (Abb. 2.8B). Neben der Molmassenverteilung führt die ESI-MS bei synthetischen Polymeren also zusätzlich auch noch zu einer Ladungsverteilung der Ketten, was die Interpretation der Spektren vor allem von Polymeren mit hoher Molmasse erschwert. ${ }^{[18[19]}$ Bei Biopolymeren kann die Mehrfachladung sogar von Vorteil sein, da sie es erlaubt hochmolekulare Ver-

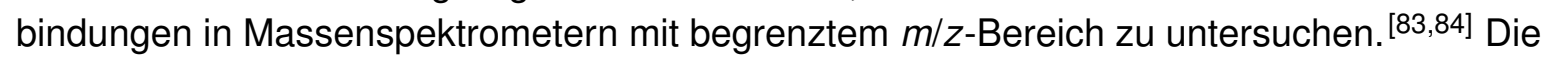
Anzahl der Ladungen einer Polymerkette kann über die $m / z$-Differenz der ${ }^{13} \mathrm{C}$-Isotope $(+\simeq$ $1,++\simeq 0,5$ etc.) oder der Polymerketten bestimmt werden ( $+=$ Masse Monomer, $++=$ halbe Masse des Monomers). Die mittels Massenspektrometrie erhaltenen Molmassenverteilungen sind in der Regel gegenüber denen aus der Gelpermeationschromatographie (GPC) zu kleineren Molmassen verschoben. Hauptursache hierfür sind verschiedene Massendiskriminierungseffekte in den Massenspektrometern. So ist die Transporteffizienz und die Detektorsensitivität von schwereren Molekülen in Massenspektrometern geringer. ${ }^{[70 \mid 71 / 85}$ Bei der MALDI-MS kann auch die Probenpräparierung einen Einfluss auf die Massendiskriminierungseffekte aufweisen und zudem ist die Überführung von höher molekulare Ketten in die Gasphase erschwert. 70171

Da es den meisten synthetischen Polymeren an basischen Gruppen mangelt, werden sie nicht durch Protonierung, sondern wie bereits erwähnt durch komplexierung mit Metallionen ionisiert. Bei den sauerstoffreichen (Meth-)Acrylaten werden meist Alkalimetallionen 

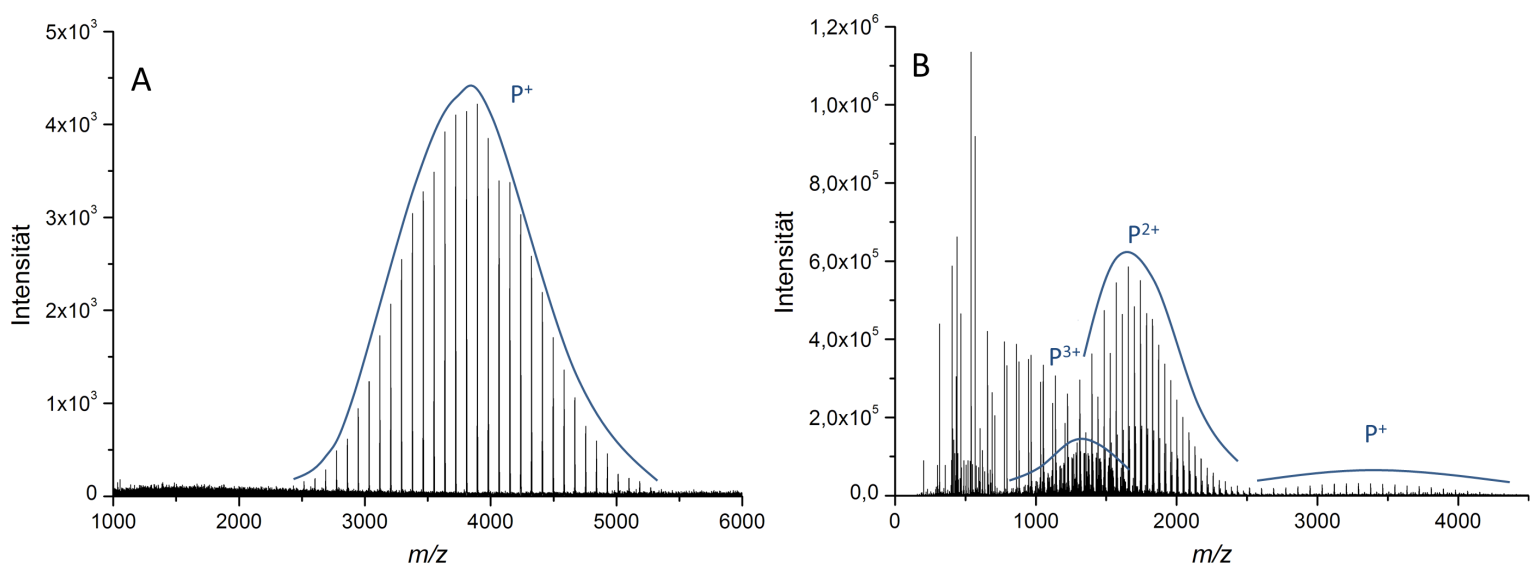

Abb. 2.8. MALDI (A) und ESI (B) Massenspektrum einer Poly(Methylacrylat) (PMA) Probe mit einer mittleren Molmassen $M_{n}=4 \mathrm{~kg} \mathrm{~mol}^{-1} . \mathrm{P}^{+}, \mathrm{P}^{2+}$ und $\mathrm{P}^{3+}$ markieren die Verteilung von einfach, doppelt und dreifach geladenen Polymerketten.

verwendet. Bei unpolaren Verbindungen wie Polystyrol (PS) werden Übergangsmetallionen wie $\mathrm{Ag}^{+}$oder $\mathrm{Cu}^{+}$verwendet, da diese durch die $\pi$-Elektronen der Styroleinheit komplexiert werden können. 18/19|70 Die hohe Massenauflösung der Massenspektrometer erlaubt es einzelne Ketten der Molmassenverteilung eines Polymers zu untersuchen (Abb. 2.8A/B). Je nach Gerät und Molmasse des Polymers kann auch das Isotopenmuster einer Polymerkette aufgelöst werden.

Die Massenspektrometrie ermöglicht die Bestimmung der exakten Molmasse und der Endgruppen von Polymeren mit nur einer Messung. Zudem ist sie wesentlich sensitiver und zeitsparender als die GPC in Verbindung mit dem klassischen Brechungsindexdetektor. Daher erlaubt die Massenspektrometrie auch die schnelle Untersuchung von kleinsten Spuren einer Polymerprobe. Im Gegensatz zur GPC ist die MS eine absolute Methode. Die MS ist daher trotz der erwähnten Massendiskriminierungseffekte die genauere Methode bei Polymeren, deren Mark-Houwink-Koeffizienten nicht bekannt sind. 18 [19] Kernspinresonanz(NMR) und Infrarotspektroskopie werden verwendet, um die makroskopische Zusammensetzung von Copolymeren zu bestimmen. Mit der MS kann die Zusammensetzung einzelner Polymerketten auf molekularer Ebene bestimmt werden. 20[21]

\subsubsection{Ladungsreduzierung der Polymerketten in der ESI-MS}

Wie bereits in Abschnitt 2.3.3 erwähnt, ist es von Vorteil für die Interpretation der Massenspektren nur einfach geladenen Ketten zu erhalten oder zumindest die Ladungsverteilung in der ESI-MS zu reduzieren. Mehrere Methoden sind in der Literatur beschrieben, um eine Ladungsreduzierung in der ESI-MS zu erhalten. Ein Teil der Ladungen der Analytmoleküle aus dem ESI-Spray kann mittels einer Coronaentladung entgegengesetzter Spannung oder durch lonen, die durch einen $\alpha$-Strahler $\left({ }^{210} \mathrm{Po}\right)$ erzeugt wurden, neutralisiert werden (Abb. 2.9. [86-90] Die Coronaentladung hat dabei den Vorteil, dass keine radioaktive Quelle benötigt wird und dass das Ausmaß der Ladungsreduzierung durch die variable Spannung einge- 
stellt werden kann. Eine Photoionisationsquelle und die Zugabe eines geeigneten Dopantmoleküls können ebenfalls zur Ladungsreduzierung der lonen innerhalb des Elektrosprays verwendet werden. [91] Mit diesen Methoden ist es möglich einfach geladene Ketten des Po-

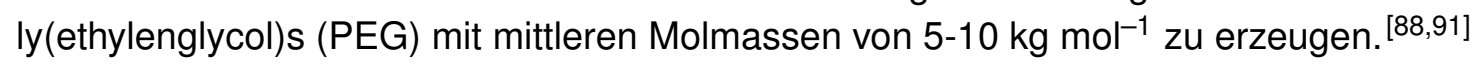

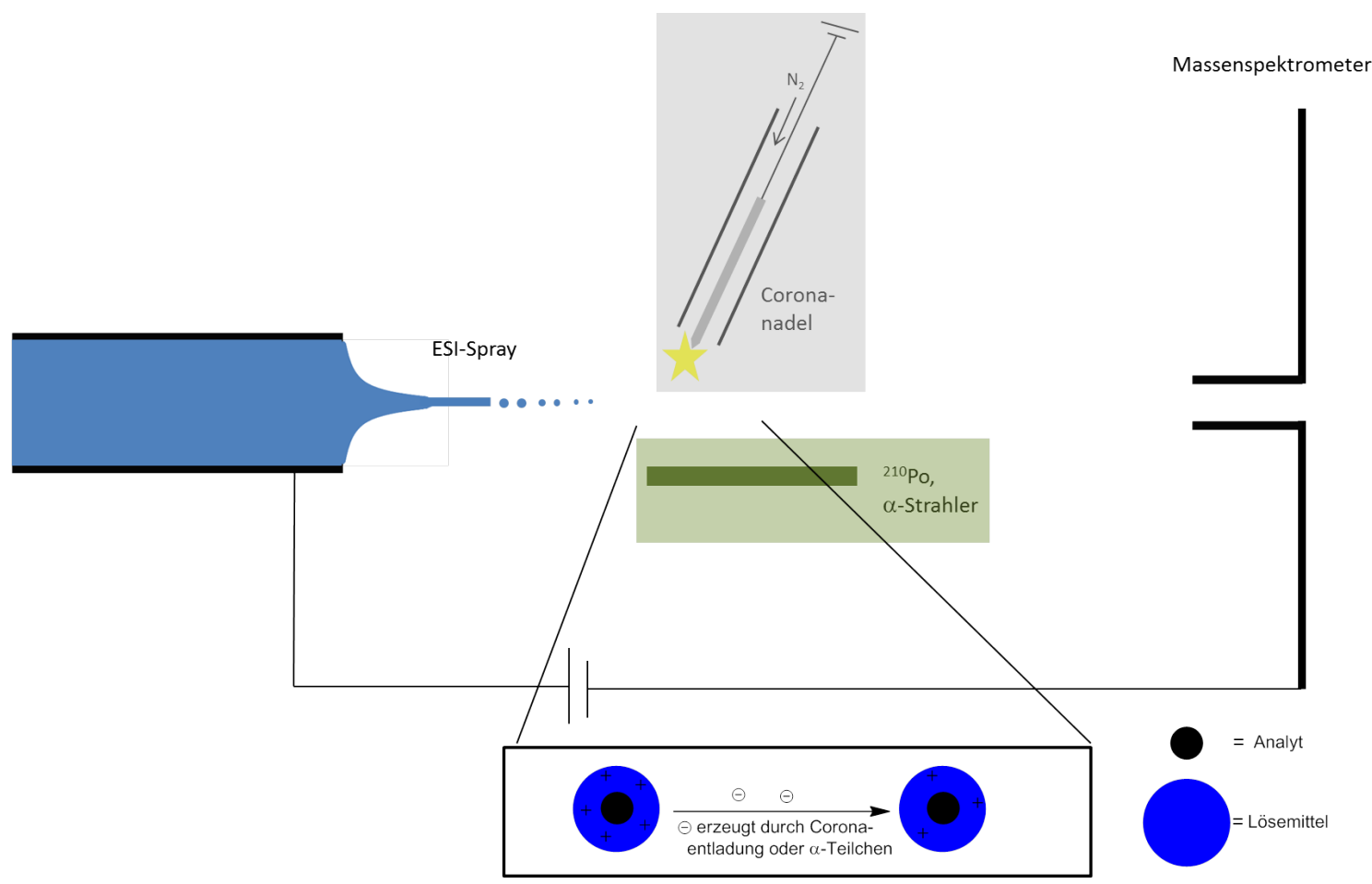

Abb. 2.9. Ladungsreduzierung der Analyten im ESI-Spray: Mit Hilfe einer Coronaentladung einer gegensätzlichen Spannung (grau hinterlegt) oder mit Hilfe von lonen erzeugt aus Stößen mit $\alpha$ Teilchen (grün hinterlegt).

Des Weiteren wurde die Ladungsreduzierung von Analytionen durch Reaktion von mehrfach geladenem PEG mit Fluorkohlenwasserstoffen und lodidionen oder mit Kronenethern in einer Quadrupolionenfalle erreicht. ${ }^{9293]}$ Auch die Zugabe von tertiären Aminen und quartären Ammoniumverbindungen können die Anzahl der Ladungen und Ladungsverteilungen in PEG reduzieren. ${ }^{94 \mid 95]}$ Alle hier genannten Methoden beschränken sich bei der Untersuchung von synthetischen Polymeren auf PEG. Im Falle der Zugabe von quartären Ammoniumverbindungen funktioniert die Ladungsreduzierung nur bei Polyether. 95

\subsubsection{Massenspektrometrie von Polyethylen}

Polyethylen (PE) und andere Polyolefine stellen in der massenspektrometrischen Analyse immer noch eine Herausforderung dar, da sie keine polaren Gruppen besitzen. 2223] Folglich ist deren lonisierung mittels Komplexierung von Metallionen oder Protonierung nicht möglich. Eine Möglichkeit Polyolefine dennoch mittels Massenspektrometrie zu untersuchen und so die in Abschnitt 2.3.3 erwähnten Vorteile des MS auch für PE zu verwenden - ist der Einbau von polaren Gruppen in die Polymerkette. Je nach Art der polaren Gruppe kann das 
Polymer dann durch Protonierung oder Metallionkomplexierung ionisiert werden. 2496] Auch vinylterminierte (z.B. durch $\beta$-Hydrideliminierung oder durch Kettentransfereliminierung) Polyolefine können aufgrund der $\pi$-Elektronen mittels $\mathrm{Ag}^{+}$ionisiert werden. [22123197] Zudem gibt es auch Veröffentlichungen, die die lonisierung von PE mittels Übergangsmetallionen erlauben, auch wenn keine Vinylgruppe vorhanden ist. ${ }^{98]}$ Alle erwähnten Literaturstellen verwenden die MALDI-MS zur Adduktionisierung von PE.

Eine weitere Methode ist die direkte Einführung einer ionischen Endgruppe. PE mit kationischer Endgruppe ist zudem auch mit der ESI-MS untersuchbar. 99-101] Die Synthese eines gut untersuchten kationischen PE ist in Schema 2.5 gezeigt. Vinylterminiertes PE wurde mittels $\mathrm{HBr}$ und einem Peroxid vorzugsweise zum anti-Markovnikov-Produkt hydrobromiert. Anschließend wurde das Brom mit Triphenylphosphin substituiert, sodass ein PE mit einer Phosphoniumendgruppe erhalten wurde. ${ }^{97 / 102 / 103}$ Die kationische Endgruppe in PE ermöglicht auch weitere Anwendungen außerhalb der Massenspektrometrie. So wurde gezeigt, dass sich kurzkettiges PE mit Ammonium- und Phosphoniumendgruppen als Phasentransferkatalysator (PTK) eignet. Der PE-PTK lässt sich nach der Reaktion durch Abkühlen und Filtration des Reaktionsgemisches zurückgewinnen, da $\mathrm{PE}$ unterhalb von $80^{\circ} \mathrm{C}$ unlöslich ist. 104-106

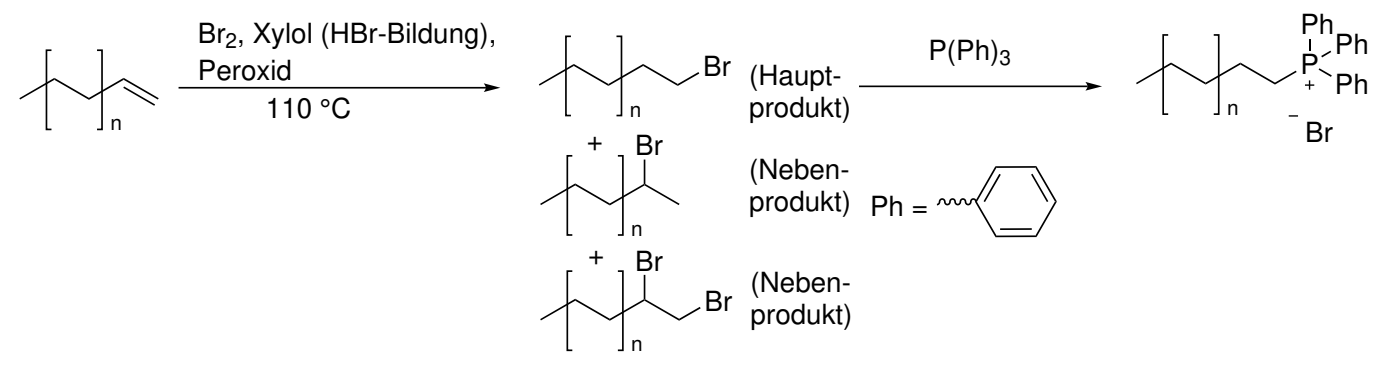

Schema 2.5. Synthese von PE mit einer Phosphoniumendgruppe.

\subsection{Blockcopolymere}

Diblockcopolymere bestehen aus einem Block des Monomers A und einem Block des Monomers B. Aufgrund der chemischen Inkompatibilität, die aus der Entmischungsenthalpie resultiert, sind die Blöcke A und B oftmals nicht mischbar. Während sich Mischungen der Homopolymere makroskopisch entmischen, führt die Mikrophasenseparation (MPS) in Blockcopolymeren (BCP) zu verschiedenen Morphologien mit Phasengrößen von 5 bis $100 \mathrm{~nm}$. 107 Grund dafür ist, dass die Blöcke in BCP untereinander kovalent verknüpft sind. Mikrophasenseparierte BCP finden als Schablone in der Lithographie und als Nanostempel sowie als optische Schaltsensoren und Datenspeicher Anwendung. 108-112

Ob MPS auftritt und welche Morphologie entsteht ist von drei Parametern abhängig:

1. dem Flory-Huggins-Parameter $\chi$,

2. dem Polymerisationsgrad $n$, 
3. dem Volumenbruch der Blöcke A und B.

Der Flory-Huggins-Parameter ist antiproportional zur Temperatur und enthält die Wechselwirkung zwischen den Blöcken A und B. Er wird durch Gleichung 2.2 beschrieben.

$$
\chi=\left(\frac{y}{k_{\mathrm{b}} T}\right)\left[\epsilon_{\mathrm{AB}}-\frac{1}{2}\left(\epsilon_{\mathrm{AA}}-\epsilon_{\mathrm{BB}}\right)\right]
$$

Hierbei ist $y$ die Koordinationszahl, also die Anzahl an direkten Nachbarn, $k_{\mathrm{b}}$ die Boltzmannkonstante, $T$ die Temperatur und $\epsilon_{\mathrm{AB}}, \epsilon_{\mathrm{AA}}$ sowie $\epsilon_{\mathrm{BB}}$ stellen die Wechselwirkungsenergien zwischen einer Monomereinheit $A$ und $B, A$ und $A$ oder $B$ und $B$ dar. Je höher der $\chi$-Parameter, um so größer ist die Inkompatibilität der zwei Blöcke.

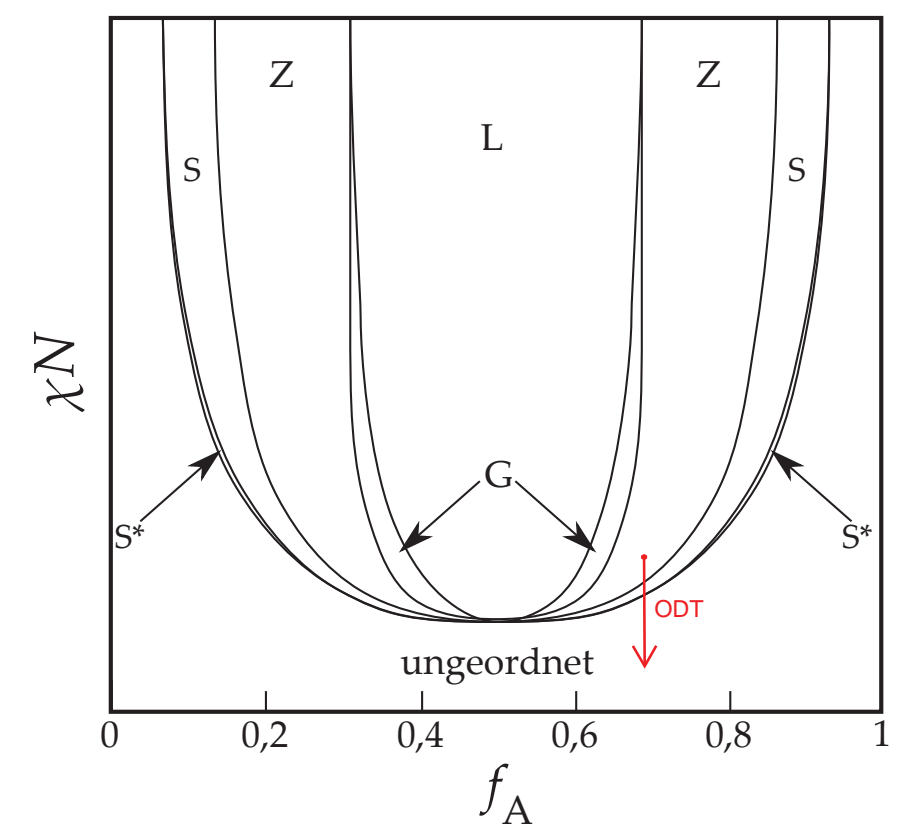

Abb. 2.10. Theoretisches Phasendiagramm für ein AB-Blockcopolymer. Es treten folgende Morphologien auf: Sphären (S), Gyroide (G), hexagonale Zylinder (Z), Lamellen (L) und kubisch dichteste Sphären $\left(S^{*}\right)$. Die Abbildung ist an die Literatur angelehnt. 113

Abbildung 2.10 zeigt das theoretische Phasendiagramm eines Blockcopolymers, bei dem die Monomere A und B die gleiche Größe, Form und Kuhnlänge besitzen. 113-115] Die Kuhnlänge ist ein Maß für die Steifigkeit des Polymers. In Abhängigkeit des Produktes $N \chi$ und des Volumenbruches $f_{\mathrm{A}}$ werden unterschiedliche Morphologien erhalten. Die verschiedenen Morphologien sind in Abbildung 2.11 grafisch dargestellt. Sind die Monomere unterschiedlich in Form, Größe oder Kuhnlänge, so geht die Symmetrie um $f_{\mathrm{A}}=0,5$ verloren. Entsprechend kann bei gegebenem Volumenbruch die Morphologie unterschiedlich zu der des theoretischen Phasendiagramms sein. Bei hohen und niedrigen Werten von $f_{\mathrm{A}}$ sowie unterhalb der Phasengrenzen (Abb. 2.10) sind beide Blöcke mischbar. Das System ist ungeordnet und es tritt nur eine Phase auf. Durch Temperaturerhöhung kann in manchen Blockcopolymeren eine Phasenübergang vom geordneten in den ungeordneten Bereich stattfinden, da der $\chi$-Parameter temperaturabhängig ist. Diese Übergang wird dementsprechend 


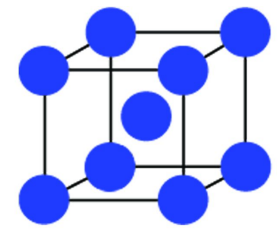

Sphären

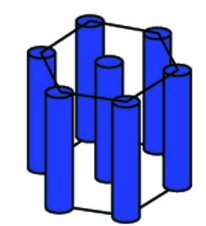

hexagonale Zylinder

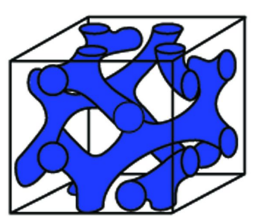

Gyroid

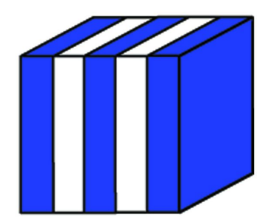

Lamellen

Abb. 2.11. Die Strukturen der Sphären (S), Gyroide (G), hexagonalen Zylinder (Z), Lamellen (L) und kubisch dichtesten Sphären $\left(S^{*}\right)$, die entsprechend des theoretischen Phasendiagramms für ein AB-Blockcopolymer erhalten werden können. Die Abbildung ist an die Literatur angelehnt. 116

Ordnungs-Unordnungs-Übergang (im englischen order-disorder-transition, ODT) genannt.

In einem symmetrischen Blockcopolymer findet der ODT bei $N \chi \simeq 10,5$ statt. Des Weiteren wird noch zwischen dem weak (WSR) und strong segregation region (SSR) unterschieden. ${ }^{117}$ Beim WSR ist $N \chi$ nur geringfügig größer als 10,5 und die Unverträglichkeit der Blöcke ist gering. Beim SSR ist $N \chi$ wesentlich größer, die Unverträglichkeit nimmt zu und die Phasengrenze zwischen den Blöcken wird kleiner. Die Morphologie der BCPs ist ein Zusammenspiel aus zwei Effekten. Um die Enthalpie zu minimieren, wird eine möglichst kleine Grenzfläche angestrebt. Um eine möglichst hohe Entropie zu erreichen, sollten die Ketten im idealen Gauß'schen Knäuel vorliegen. Durch die MPS werden die Ketten aber teils gestreckt. Abbildung 2.12 zeigt die Phasengrenze eines Blockcopolymers mit zunehmendem Anteil $f_{\mathrm{A}}$ (Zunahme des orangen Blocks). Bei unsymmetrischen A- und B-Blöcken ist die Phasengrenze gekrümmt, es liegen Sphären oder Zylinder des Blocks $A$ in der Matrix des Blocks B vor. ${ }^{[116]}$ Bei symmetrischen Blöcken (lamellare Struktur) ist die Phasengrenze nicht gekrümmt.
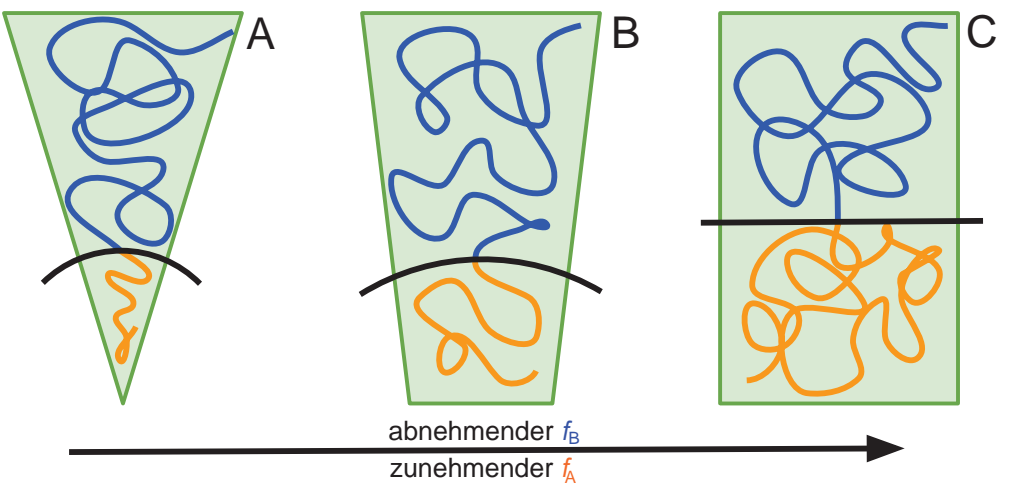

Abb. 2.12. Schematisch Darstellung der Phasengrenze bei einer sphärischen (A) und zylindrischen (B) sowie bei einer lamellaren Struktur (C). Die Abbildung ist an die Literatur angelehnt. ${ }^{116}$ 


\subsection{Zugversuch}

Je nach molekularer Zusammensetzung weisen Polymere eine unterschiedliche Steifig- und Dehnbarkeit auf. Diese mechanischen Eigenschaften können unter anderem anhand des Zugversuchs ermittelt werden und bestimmen das Anwendungsfeld des Polymers. 118]

Im Zugversuch wird ein rechteckiger oder knochenförmiger Probenkörper des zu untersuchenden Materials an beiden Enden der Längsachse mithilfe zweier Klammern in die Zugmaschine eingespannt. Der Probenkörper wird dann mit einer konstanten Geschwindigkeit entlang der Längsachse bis zum Bruch gedehnt, während die zur Dehnung benötigte Kraft gemessen wird. 1191120] Die relative Dehnung $\epsilon$ ist entsprechend Gleichung 2.3 definiert. Hierbei ist $l$ die aktuelle absolute Länge und $l_{0}$ die anfängliche Länge des Probenkörpers.

$$
\epsilon=\frac{\left(l-l_{0}\right)}{l_{0}}
$$

Die Kraft $F$ dividiert durch die anfängliche Querschnittsfläche $A_{0}$ an der dünnsten Stelle des Probenkörpers wird nominelle oder auch technische Zugspannung $\sigma$ genannt (Gleichung 2.4). Die Querschnittsfläche ändert sich während des Zugs. Trotzdem wird die Kraft durch die anfängliche Querschnittsfläche geteilt, da die wahre Querschnittsfläche bei der jeweiligen Dehnung nicht bekannt ist. Die wahre Zugspannung ist nach beginnender Dehnung immer größer als die nominelle Zugspannung. In dieser Arbeit wird nur die technische Zugspannung verwendet.

$$
\sigma=\frac{F}{A_{0}}
$$

In einem Spannungsdehnungsdiagramm wird die Zugspannung gegen die Dehnung aufgetragen. Die Zugkurve eines thermoplastische und zähen Polymers ist in Abbildung 2.13 gezeigt. Im Anfangsbereich besteht bei allen Materialien ein linearer Zusammenhang zwischen der Zugspannung und Dehnung (Gleichung 2.5). Die Proportionalitätskonstante ist ein Materialkennwert und wird auch Elasizitäts- , Young- oder einfach nur E-Modul genannt. Je höher der E-Modul, umso steifer ist das Material.

$$
\sigma=E \epsilon
$$

Die Streckgrenze $R_{\mathrm{e}}$ markiert den Punkt, bis zu dem eine Dehnung vollständig reversibel ist. Oberhalb der Streckgrenze findet eine plastische und permanente Formveränderung statt. Starke Materialien haben eine hohe Streckgrenze und spröde Materialien brechen nahe der Streckgrenze. Die Zugfestigkeit $R_{\mathrm{m}}$ ist die maximale Spannung, mit der ein Werkstoff belastet werden kann bevor er bricht. Die Bruchdehnung $\epsilon_{\mathrm{B}}$ gibt die maximale Dehnung, also die Dehnung beim Bruch, an. Duktile Materialien lassen sich plastisch verformen und haben eine hohe Bruchdehnung. Wichtig ist außerdem die Zähigkeit $Z$, die Fläche unter der Zugkurve. Sie ist ein Maß für die Energie ist, die ein Probenkörper bis zum Bruch aufnehmen kann. Die Zähigkeit ist ein Zusammenspiel aus Duktilität und Stärke des Materials.

Der Verlauf der Zugkurve ist abhängig von der Temperatur, [119] der Zusammensetzung, der molekularen Struktur, der molaren Masse ${ }^{[131121]}$ und den Prozessbedingungen ${ }^{[122]}$ des 


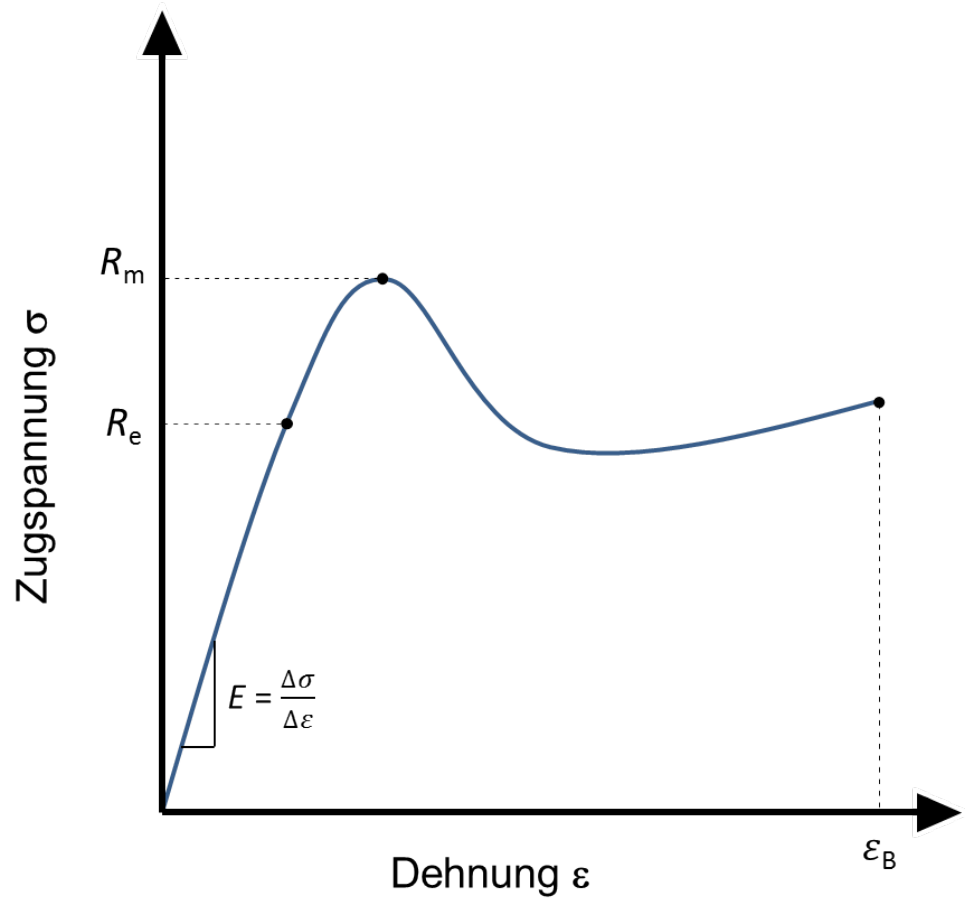

Abb. 2.13. Eine Zugkurve eines thermoplastischen und zähen Polymers.

Polymers sowie von den Messbedingungen. ${ }^{118}$ Vernetzung erhöht den E-Modul und die Zugfestigkeit unterhalb und im Bereich der Glasübergangstemperatur. Die Bruchdehnung und das Kriechverhalten werden hingegen reduziert. ${ }^{9}$

\subsection{Reversible Photovernetzung}

Vernetzung von Polymeren wird aus verschiedenen Gründen durchgeführt. Die Vernetzung führt oftmals zu einem größeren $E$-Modul, einer höheren Härte sowie einer erhöhten chemischen und thermischen Stabilität des Polymers. ${ }^{9110]}$ Auch sind Elastomere, gekennzeichnet durch die Gummielastizität, nur durch leichte Vernetzung der Ketten zugänglich. 101123] Eine Möglichkeit vernetzte Polymere zu erhalten ist der Einsatz von mindestens trifunktionellen Monomeren. In diesem Fall findet die Vernetzung während der Polymerisation statt und eine nachträgliche Veränderung der Form des Probenkörpers ist ebenso wenig möglich wie eine Charakterisierung des Polymers mittels GPC. Das Polymer kann aber auch nach der Polymerisation vernetzt werden. Dies gelingt z.B. durch die Reaktion von Epoxiden in der Seitenketten der Polymere mit einem multifunktionellen Amin oder bei der Vulkanisation durch die Reaktion von Schwefel mit Doppelbindungen des Polymers. ${ }^{[124}$ Des Weiteren ist eine nachträgliche Vernetzung durch den Einsatz von Peroxiden oder durch hochenergetische Strahlung möglich. 10[123|125] In all diesen Fällen ist die Vernetzung irreversibel.

Bei Derivaten der Zimtsäure, 126-128 des Dimethylmaleimids, 129-131] des Coumarins ${ }^{[132[133]}$ basiert die reversible Photovernetzung auf der reversiblen [2+2] Cycloadditi- 


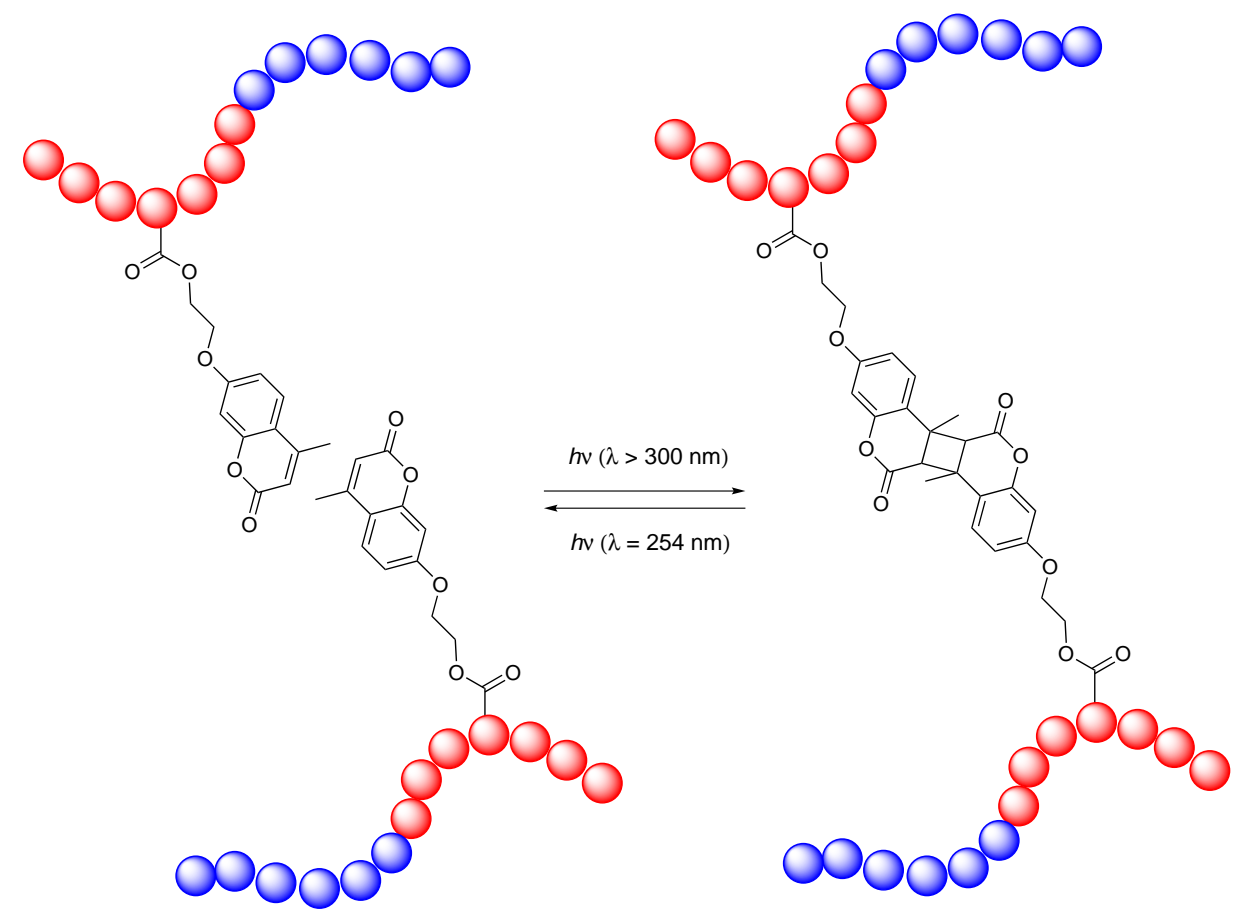

Abb. 2.14. Schematische Darstellung der Photover- und entnetzung eines Blockcopolymers mit Cumaringruppen in der Seitenkette des roten Blocks.

on. ${ }^{134]}$ Diese Derivate können als Comonomer eingesetzt werden. Bei Zimtsäure und seinen Derivaten konkurriert die Photovernetzung mit der cis/trans-Isomerisierung. Zudem ist die Doppelbindung anfällig für radikalische Reaktionen. ${ }^{129]}$ Die Photovernetzung ist in Abbildung 2.14 für ein Coumarin enthaltendes Blockcopolymer schematisch dargestellt. Das Coumarinmonomer wurde durch Copolymerisation im roten Block eingebaut. Liegen zwei Coumaringruppen zweier verschiedener Polymerketten nah nebeneinander kann durch Bestrahlung mit Licht der Wellenlänge $(\lambda>300 \mathrm{~nm})$ die [2+2]-Cycloaddition zur Vernetzung führen. Anschließend kann das Polymer durch Verwendung eine kürzeren Wellenlänge $(\lambda=254 \mathrm{~nm})$ wieder entnetzt werden. Die Begriffe „Entnetzung“ oder „entnetzen“ werden in dieser Arbeit zur Beschreibung der Rückreaktion der Vernetzung verwendet. Im Allgemeinen wurde in der Literatur die Photovernetzung besser untersucht als die Spaltung des Netzwerks. [135] Aufgrund der energiereichen Strahlung können während der Entnetzung Nebenreaktionen auftreten, die die erneute Photovernetzung beeinflussen können. ${ }^{[136]}$ Zudem wird die energiereiche Strahlung von vielen funktionellen Gruppen im Polymer absorbiert, weshalb die Eindringtiefe im Polymer geringer ist.

Derivate des Anthracens können ebenfalls als Comonomer und Photovernetzer eingesetzt werden. $137-140$ In diesem Fall basiert die reversible Photovernetzung auf einer [4+4] Cycloaddition, wie in Abbildung 2.15 für ein in 9-Position substituiertes Anthracen gezeigt. 141] Anthracenmonomere können radikalisch aber nur mit Methacrylaten copolymerisiert werden. Die Polymerisation von Acrylaten und Styrolen wird inhibiert. [139[142] Grund dafür ist, dass Anthracen ein guter Radikalfänger ist, da es durch H-Abstraktion ein diben- 
zylisches Radikal bilden kann. Dieses ist zu stabil, um die Polymerisation von Acrylaten zu initiieren. Lediglich wenn die 10-Position des Anthracens mit einer Alkylgruppe substituiert ist, ist eine Copolymerisation mit Acrylaten möglich. 143/144] Obwohl die [4+4] Cycloaddition und -reversion nach den Woodward-Hoffmann-Regeln nur photochemisch erlaubt sind, findet die Cycloreversion bei Anthracen sowohl photochemisch als auch thermisch statt. [141] Die photochemische Dimerspaltung erwies sich in der Literatur jedoch weniger effektiv als die thermische. ${ }^{|137 / 138| 141 / 145]}$ Dabei wurde gezeigt, dass der Substituent an $C_{9}(R$ in Abb. 2.15 einen großen Einfluss auf die für die Cycloreversion benötigte Temperatur hat. ${ }^{141]}$ Die thermische Netzwerkspaltung führt vermutlich zu weniger Nebenreaktionen als die photochemische Spaltung und ist in der Eindringtiefe nicht begrenzt.

Die reversible Photovernetzung wurde bereits in dünnen Filmen, Mizellen ${ }^{[138}$ und $\mathrm{Hy}$ drogelen ${ }^{[45]}$ untersucht. Sie wird auch in selbstheilenden und Formgedächtnis-Materialien eingesetzt. $146-149$<smiles>[R]c1c2ccccc2cc2ccccc12</smiles>

Abb. 2.15. Schematische Darstellung der reversiblen [4+4] Cycloaddition des an der 9-Position substituierten Anthracens. Es ist das Kopf-Schwanz-Dimer gezeigt, das Kopf-Kopf-Dimer ist aber ebenfalls ein mögliches Produkt. 


\section{Kationische Endgruppen in PE}

Dieses Projekt wurde in einer engen Kooperation mit Jannik Wagner durchgeführt. [150] Der Fokus des Autors dieser Arbeit lag auf der Analyse, während der Fokus von Herrn Wagner auf der Synthese lag. Nichtsdestotrotz wird hier das vollständige, gemeinschaftliche Projekt vorgestellt. Dieser Teil der Arbeit wird in ähnlicher Weise auch in Jannik Wagners Dissertation zu finden sein.

\subsection{Motivation}

Polyolefine - dabei vor allem Polyethylen und Polypropylen - besitzen einen Marktanteil von über $50 \%$ am globalen Kunststoffmarkt und sind daher von großer Bedeutung in Industrie und Forschung. ${ }^{[151 \mid 152]}$ Ein Augenmerk liegt dabei vor allem auf der Modifikation der Endgruppe. Die klassischen Polymerisationsverfahren zur Herstellung von Polyolefinen bieten nur sehr begrenzte Möglichkeiten die Endgruppen zu modifizieren und liefern zudem Polymere mit hoher Dispersität. Die catalyzed chain growth (CCG) Polymerisation verwendet ein metallorganisches Kettentransferreagenz, welches am Ende der Polymerisation Teil der Polymerkette ist. Dadurch ist es möglich mittels metallorganischer Reaktionen die Endgruppe des Polymers zu modifizieren. Ein weiterer Vorteil ist die enge Molmassenverteilung der resultierenden Polymere. 24/34/35] Aufgrund ihrer technischen Bedeutung ist eine vollständige Charakterisierung der Polyolefine wichtig. Massenspektrometrie erlaubt neben der Bestimmung von Molmassen auch die Bestimmung der Endgruppe. Jedoch können unmodifizierte Polyolefine massenspektrometrisch nicht untersucht werden, da im Polymer keine für die Ionisation mit Protonen oder Metallionen benötigten polaren Bindungsstellen zur Verfügung stehen. 22223]

Ziel dieses Projektes ist das Einbringen einer polaren und ionischen Endgruppe in PE mittels CCG-Polymerisation. Dazu soll zunächst durch eine metallorganische Reaktion mit elementarem lod eine lodendgruppe eingeführt werden, da diese Reaktion sehr effizient ist und eine Vielzahl weiterer Funktionalisierungen ermöglicht. [24] Im zweiten Schritt soll mittels nucleophiler Substitution des lodatoms mit einem tertiären Amin eine Ammoniumendgruppe eingeführt werden.

Des Weiteren soll untersucht werden, inwiefern

1. die kationische Endgruppe eine massenspektrometrische Analyse von PE erlaubt.

2. durch die ionische Endgruppe spezielle Anwendungsmöglichkeiten aufgrund ihrer Phasentransfereigenschaften möglich sind. ${ }^{[153[154]}$, z.B. als Phasentransferkatalysator (PTK) oder zum Einbau von anionischen, polaren Verbindungen. Möglich wäre das $P E$ als Phasentransferkatalysator einzusetzten oder anionische polare Verbindungen einzubauen, die ansonsten nicht mischbar mit PE sind. 104-106 
3. es möglich ist, das geladene PE weiter zu funktionalisieren. Das Amin in der oben beschriebenen Synthese wird daher so gewählt, dass eine weitere Funktionalität für anschließende Reaktionen zur Verfügung steht. Nach bestem Wissen des Autors dieser Arbeit verfügt bisher kein in der Literatur beschriebenes PE mit ionischer Endgruppe über eine weitere und zur Modifikation des Polymers geeignete funktionelle Gruppe.

\subsection{Synthese und Charakterisierung von PE mit tertiären Amin- und Ammoniumendgruppen}

Die Herstellung von PE mit einer Ammonium- oder tertiären Aminendgruppe ist in Schema 3.1 gezeigt. Dabei wurde PE mit $\left(\mathrm{C}_{5} \mathrm{Me}_{5}\right)_{2} \mathrm{NdCl}_{2} \mathrm{Li}(\mathrm{OEt})_{2}$ als Prekatalysator und Butyloctylmagnesium als Aktivator und Kettentransferreagenz mittels CCG polymerisiert. Anschließend wurde das Dipolyethylenmagnesium (PE-Mg-PE) mit lod in einer äußerst effizienten Reaktion zu lod-endfunktionalisiertem PE (PE-I) umgesetzt. Für die Herstellung von PE mit teriärer Amin- (PE-N) oder Ammoniumendgruppe (PE- $\mathrm{N}^{+}$und $\mathrm{PE}-\mathrm{N}^{+}{ }_{6}$ ) wurde das lod mit einem Überschuss von mindestens drei Äquivalenten (äq) 1-(Methylamino)ethanol, 3-Dimethylamino-1-propanol oder 6-Dimethylamino-1-hexanol nucleophil substituiert. Der Unterschied zwischen PE- $\mathrm{N}^{+}$und $\mathrm{PE}-\mathrm{N}^{+}{ }_{6}$ liegt in der Länge der Alkylkette zwischen der Ammonium- und der Hydroxylgruppe. Diese Variierung wurde durchgeführt, um den Einfluss des Abstandes der positiven Ladung zur Hydroxylgruppe bei weiteren Funktionalisierungsreaktion von $\mathrm{PE}-\mathrm{N}^{+}$zu untersuchen (siehe dazu Abschnitt 3.5). Die Charakterisierung der Polymere wird hauptsächlich anhand von PE-N und PE- $\mathrm{N}^{+}$diskutiert. Sie ist für PE- $\mathrm{N}^{+}{ }_{6}$ aber analog zu PE-N+.

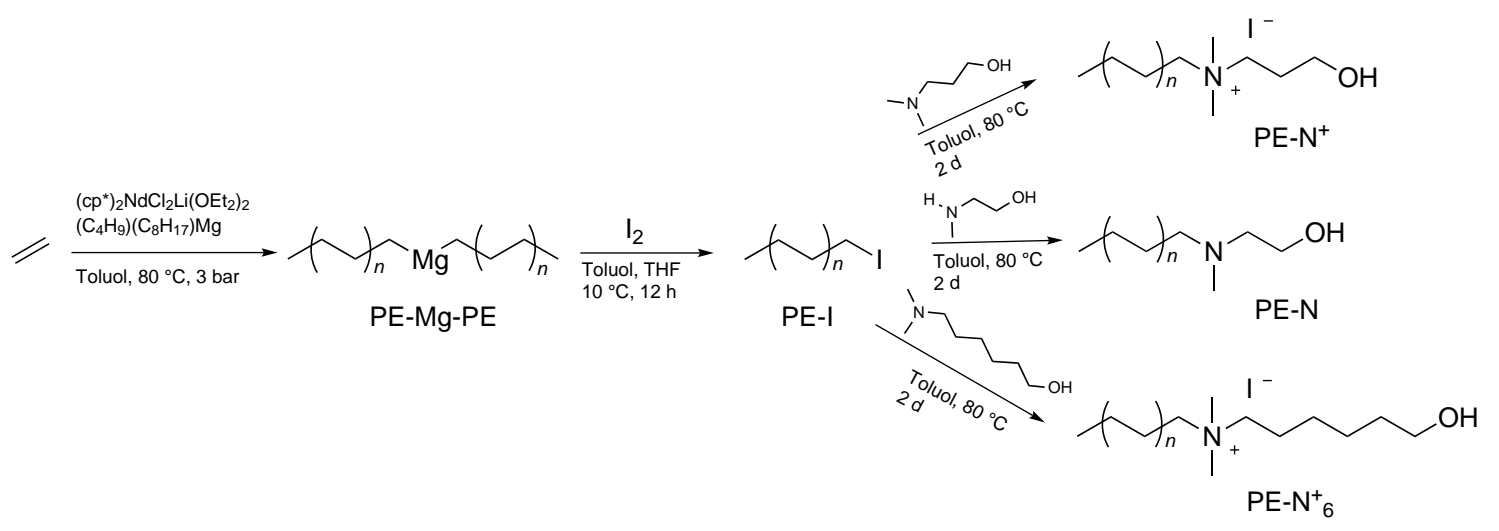

Schema 3.1. Synthese von PE mit tertiärer Amin- oder Ammoniumendgruppe.

Abbildung 3.1 zeigt die mittels Hochtemperatur-Gelpermeationschromatogaphie (HTGPC) erhaltenen Molmassenverteilungen (MMVs) von PE-I und PE-N+. Die geringen Dispersitäten $(\Theta=1,19)$ der Polymerproben bestätigen die gute Kontrolle der Polymerisation durch den Neodymkatalysator und die Diorganomagnesiumverbindung als Kettentreansferreagenz. Die MMVs beider Polymere mit dem Peakmaximum $\left(M_{\mathrm{p}}=850 \mathrm{~g} \mathrm{~mol}^{-1}\right)$, Zahlen- 


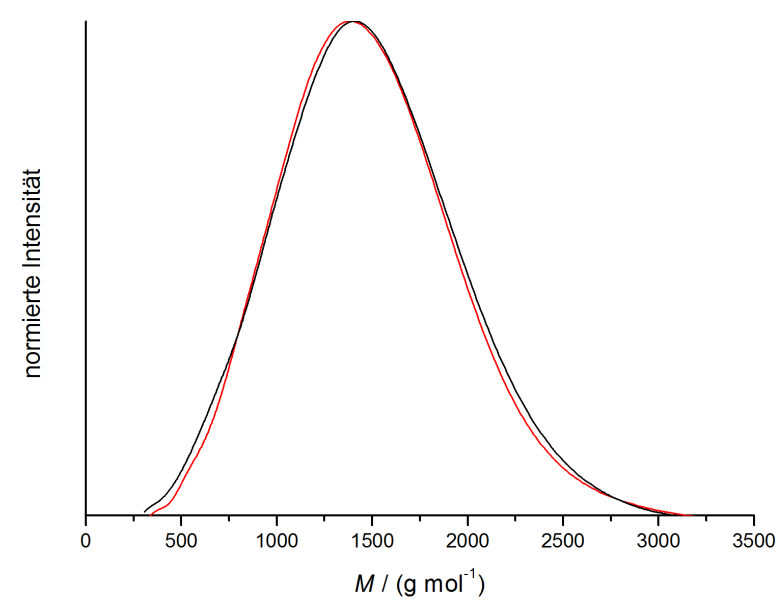

Abb. 3.1. Die Molmassenverteilungen des hergestellten PE-I (schwarz) und PE-N+ (rot).

$\left(M_{\mathrm{n}}=650 \mathrm{~g} \mathrm{~mol}^{-1}\right)$ und Gewichtsmittel $\left(M_{\mathrm{w}}=780 \mathrm{~g} \mathrm{~mol}^{-1}\right)$ der Molmasse sind nahezu identisch. Das PE- $\mathrm{N}^{+}$wechselwirkt demnach trotz der ionischen Gruppe nicht mit der stationären Phase der GPC-Säule. Die berechnete Differenz der Endgruppen von PE-I und $\mathrm{PE}-\mathrm{N}^{+}$beträgt nur $23 \mathrm{~g} \mathrm{~mol}^{-1}$ und kann aufgrund des geringen Unterschiedes und der Fehleranfälligkeit der GPC nicht mittels dieser Methode aufgelöst werden.

Um die Endgruppenfunktionalität und somit die Effizienz der nucleophilen Substitution zu bestimmen, wurden die PEs mithilfe der Protonenkernspinresonanzspektroskopie $\left({ }^{1} \mathrm{H}\right.$ NMR-Spektroskopie) untersucht. Dafür muss das Polymer vollständig gelöst sein, weshalb die Polymere in deuteriertem Toluol bei $80{ }^{\circ} \mathrm{C}$ gemessen wurden. Das PE-I hat eine lodendgruppenfunktionalität von mindestens $83 \%$ (Abb. $3.2 \mathrm{~A}$ ), die durch Vergleich des Integrals der Methylengruppe neben dem lod $\left(\mathrm{CH}_{2}-\mathrm{I}\right)$ bei $\delta=2,80 \mathrm{ppm}$ und der endständigen Methylgruppe bei $\delta=0,88 \mathrm{ppm}$ bestimmt wurde. Terminierungsreakionen mit protischen Verbindungen führen zu Polymerketten, die an beiden Enden eine Methylgruppe besitzen. Daher ist die lodendgruppenfunktionalität mindestens $83 \%$, sie kann aber auch höher sein. Protonen von vinylterminiertem PE, z.B. entstanden durch $\beta$-Hydrideliminierung, konnten praktisch nicht nachgewiesen werden (vergleiche das vollständige ${ }^{1} \mathrm{H}$-NMR-Spektrum aus dem Anhang A1). Die NMR-Spektren von PE-N, PE-N+ und PE-N ${ }_{6}^{+}$(Abb. $3.2 \mathrm{~B}-\mathrm{D}$ ) zeigen eine quantitative Substitution der lodgruppe ab einem dreifachem Überschuss des Amins, da das Signal der $\mathrm{CH}_{2}-\mathrm{I}$-Gruppe vollständig verschwunden ist.

Des Weiteren bestätigen die NMR-Untersuchungen die Struktur des amin- oder ammoniumfunktionalisierten PEs (Abb. 3.2, rechts). Das Signal bei $\delta=1,1-1,4$ ppm gehört in allen Fällen zu den Methylengruppen des Polymerrückgrats. Das charakteristische Singulett der Methylgruppe am Stickstoff erscheint bei PE-N bei $\delta=2,05 \mathrm{ppm}$. Im Vergleich dazu sind die Methylgruppen bei PE-N $\mathrm{N}^{+}$und $\mathrm{PE}-\mathrm{N}^{+}{ }_{6}$ am Ammonium wegen der elektronziehenderen Eigenschaft auf $\delta \simeq 3,4$ ppm verschoben. Die am Stickstoff und Sauerstoff gebundenen Methylengruppen (Signale d, j, und $m$ sowie $s, k$ und o) sind im Vergleich zur Methylgruppe noch weiter ins Tieffeld verschoben. Neben den zugeordneten Signalen treten keine weiteren Peaks auf, was die erfolgreiche und quantitative Endgruppenmodifizierung bestätigt. 


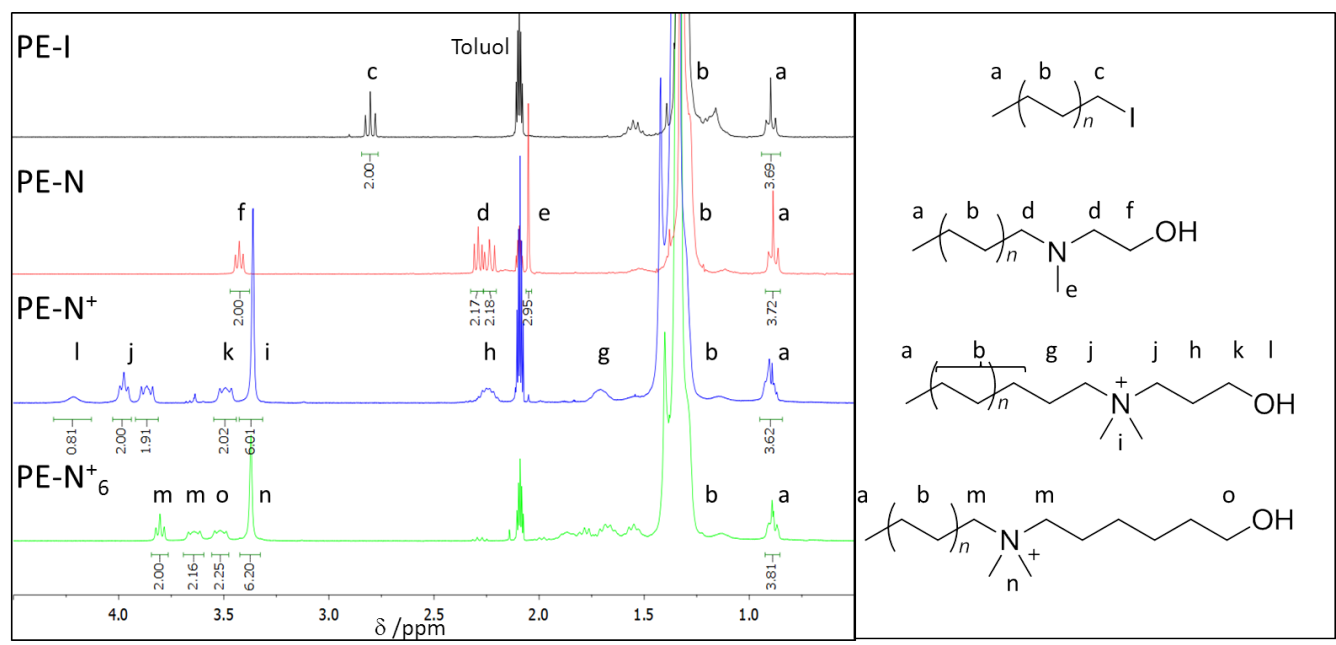

Abb. 3.2. Die ${ }^{1} \mathrm{H}-\mathrm{NMR}-\mathrm{Sp} p$ ktren von $\mathrm{PE}-\mathrm{I}, \mathrm{PE}-\mathrm{N}, \mathrm{PE}-\mathrm{N}^{+}$und $\mathrm{PE}-\mathrm{N}^{+}{ }_{6}$.

Die eingeführten ionischen und basischen Endgruppen erlauben die massenspektrometrische Analyse des Polymers, welche normalerweise aufgrund von fehlenden polaren Gruppen im PE nicht möglich ist. ${ }^{2223]}$ Die Probenpräparation ist ein entscheidender Schritt in der

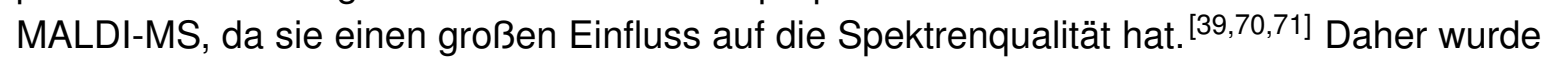
die Matrix und das Polymer-zu-Matrix-Verhältnis entsprechend optimiert. Dabei zeigte sich, dass trans-2-[3-(4-tert-Butylphenyl)-2-methyl-2-propenyliden]propandinitril (DCTB) und 2(4-Hydroxyphenylazo)benzoesäure (HABA) geeignete Matrices sind. Da PE bei Raumtemperatur unlöslich ist, wurde es zunächst bei $90^{\circ} \mathrm{C}$ in Toluol gelöst. Beim Abkühlen entstand eine feine Dispersion von PE in Toluol, die direkt mit der Matrixlösung gemischt wurde. Im Falle von PE-N wurde noch eine kleine Menge Essigsäure zur Mischung hinzugegeben, um die Protonierung der terminalen Amingruppe sicherzustellen $\left(\mathrm{PE}-\mathrm{NH}^{+}\right)$. Bei PE-I als Blindprobe wird mit beiden Matrices auch unter Zugabe von Silbertrifluoressigsäure kein PESignal in der MALDI-MS detektiert, da keine terminalen Vinylgruppen oder andere polare Gruppen im PE enthalten sind. 2223197

PE-N ${ }^{+}$und PE-N ${ }_{6}^{+}$ergeben rauscharme MALDI-Massenspektren (Abb. 3.3 A und B) mit intensiven Signalen und einem Signal-zu-Rauschverhältnis $(S / R)$ von bis zu 3000 . Dagegen beinhaltet das MALDI-Spektrum von $\mathrm{PE}-\mathrm{NH}^{+}$mehr Hintergrundpeaks und das $\mathrm{S} / \mathrm{R}$ ist geringer. Wie im Innenbild von Abb. $3 \mathrm{~A}$ zu sehen, ist der Abstand zwischen den Peaks $28 \mathrm{~g} \mathrm{~mol}^{-1}$, was der Molmasse einer Monomereinheit $\left(\mathrm{C}_{2} \mathrm{H}_{4}\right)$ entspricht. Das Isotopenmuster ist in allen Spektren aufgelöst und stimmt, wie die experimentelIen Molmassen auch, im Rahmen der Ungenauigkeit des MALDI-Spektrometers mit den berechneten überein (Abb. 3.3A und $D$ sowie Tabelle 3.1). Im Vergleich zu den Ergebnissen aus der GPC, sind in der MALDI-MS die Molmassen zu kleineren Werten verschoben. Das Peakmaximum beträgt bei PE-N ${ }^{+}$laut GPC $850 \mathrm{~g} \mathrm{~mol}^{-1}$ und im MALDI-Spektrum $637 \mathrm{~g} \mathrm{~mol}^{-1}$. Ursache für diese Verschiebung der Molmasse können die bereits im Abschnitt2.3.3 erwähnten Massendiskriminierungseffekte sein. Der Übergang 

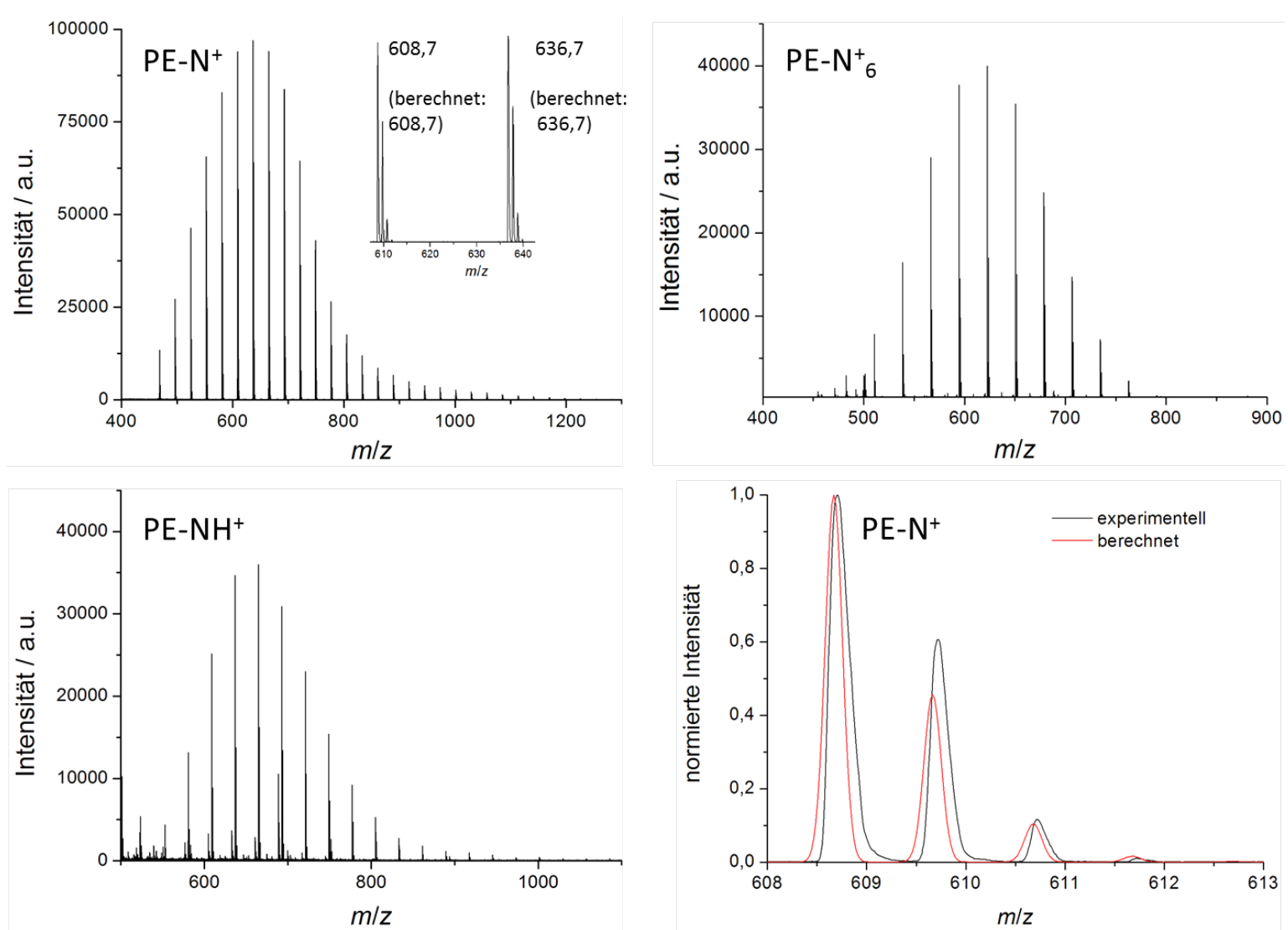

Abb. 3.3. Die MALDI-Massenspektren von PE-N ${ }^{+},{\mathrm{PE}-\mathrm{N}^{+}}_{6}$ und $\mathrm{PE}-\mathrm{NH}^{+}$sowie der Vergleich vom berechneten und experimentellen Isotopenmuster in $\mathrm{PE}-\mathrm{N}^{+}$.

Tab. 3.1. Alle gefundenen und berechneten Massen von PE mit Endgruppenmodifikationen. Die Massen beziehen sich jeweils auf das Peakmaximum.

\begin{tabular}{|c|c|c|c|c|}
\hline Methode & PE-Struktur & Formel & $m / z$ gefunden & $m / z$ berechnet \\
\hline MALD同 & $\begin{array}{c}\text { PE-N }{ }^{+} \\
\text {PE-NH } \\
\text { PE-N }{ }^{+}-M^{b} \\
\text { PE-N+-ATRP } \\
\text { PE-N+ } \\
\text { PAFT }\end{array}$ & $\begin{array}{c}\mathrm{C}_{39} \mathrm{H}_{82} \mathrm{NO}^{+} \\
\mathrm{C}_{45} \mathrm{H}_{94} \mathrm{NO}^{+} \\
\mathrm{C}_{59} \mathrm{H}_{118} \mathrm{NO}_{2}^{+} \\
\mathrm{C}_{50} \mathrm{H}_{101} \mathrm{NO}_{2}^{+} \\
\mathrm{C}_{59} \mathrm{H}_{118} \mathrm{NO}_{2} \mathrm{~S}_{3}^{+}\end{array}$ & $\begin{array}{l}608,7 \\
665,9 \\
872,4 \\
828,9 \\
968,9\end{array}$ & $\begin{array}{l}608,7 \\
665,7 \\
872,9 \\
828,7 \\
968,8\end{array}$ \\
\hline $\mathrm{ES} \mathrm{I}^{\mathrm{e}}$ & $\begin{array}{c}\text { PE-N+ } \\
\text { PE-NH+ }\end{array}$ & $\begin{array}{l}\mathrm{C}_{41} \mathrm{H}_{86} \mathrm{NO}^{+} \\
\mathrm{C}_{39} \mathrm{H}_{82} \mathrm{NO}^{+}\end{array}$ & $\begin{array}{l}608,669 \\
580,644\end{array}$ & $\begin{array}{l}608,670 \\
580,639\end{array}$ \\
\hline
\end{tabular}

${ }^{a}$ MALDI-Ergebnisse sind auf eine Nachkommastelle gerundet.

${ }^{b}$ Geladenes PE mit ATRP Initiator.

${ }^{c}$ Geladenes PE-Makromonomer.

${ }^{d}$ Geladenes PE mit Makro-RAFT-Agensfunktion.

${ }^{e}$ ESI-Ergebnisse sind aufgrund der höheren Massengenauigkeit auf drei Nachkommastellen gerundet. 
der Polymerketten in die Gasphase ist schwieriger und die Transporteffizienz im Massenspektrometer sowie die Detektorsensitivität sinken mit zunehmender Molmasse des Analyten. Auch die Probenpräparation kann einen Einfluss auf die Massenverschiebungen in

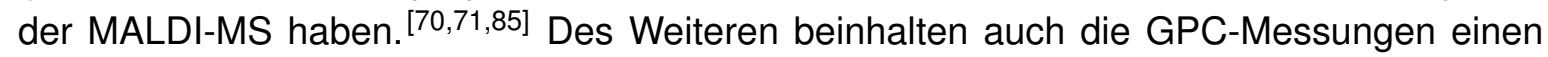
Fehler. Die GPC ist eine relative Methode und die Kalibirierung wurde mit PE-Standards hoher Dispersität durchgeführt. Zudem tragen die PE-Standards an beiden Kettenenden eine Methylgruppe. Die eingebrachten polaren Endgruppen der hier untersuchten PE-Proben können einen Einfluss auf das hydrodynamische Volumen haben, da die Molmassen der Polymere relativ klein sind. Eine weitere Möglichkeit für die Unterschiede der beiden Messmethoden könnte sein, dass der Anteil der terminierten Ketten bei höheren Molmassen zunimmt. Die terminierten Ketten tragen aber keine Amin-/Ammoniumendgruppe und können daher mittels MS nicht detektiert werden.

Die PE wurden neben MALDI auch mit ESI-MS untersucht. Die Analyse von PE mittels ESI-TOF-MS ist anspruchsvoller, da hierbei das Polymer bei Raumtemperatur in einem möglichst polaren Lösungsmittel gelöst werden muss. Durch die Verwendung einer homogenen Lösung werden in der ESI-MS aber reproduzierbarere Spektren erhalten als in der MALDIMS. PE ist allerdings unpolar und unterhalb von $80^{\circ} \mathrm{C}$ nahezu unlöslich. Deswegen wurden die PE-Proben in Dichlorethan (DCE), einem möglichst polaren Lösungsmittel, bei $90{ }^{\circ} \mathrm{C}$ gelöst. Beim Abkühlen fällt das PE als feiner Niederschlag aus. Die entstandene Dispersion wurde anschließend durch einen Spritzenfilter filtriert, um eine bei Raumtemperatur gesättigte Lösung von PE zu erhalten. Die Polarität von DCE alleine ist aber allerdings noch nicht ausreichend, um Massenspektren von PE zu erhalten. Unpolare Lösungsmittel erzeugen keinen stabilen Taylorkegel, da sie nicht genug Ladungsträger (Elektrolyten) enthalten und die elektrische Leitfähigkeit gering ist. $39 / 155-157$ Daher wurde die gesättigte Lösung von PE in DCE mit unterschiedlichen Volumina (80:20, 50:50 und 30:70) von Acetonitril (MeCN) verdünnt. Im Falle von PE-N wurde noch 0,1 wt\% Essigsäure hinzugegeben, um die terminale Amingruppe zu protonieren.

Abbildung 3.4 zeigt die ESI-Spektren von PE-N ${ }^{+}$und $\mathrm{PE}-\mathrm{NH}^{+}$in den unterschiedlichen DCE-MeCN-Mischungen. Die Intensität der PE-Signale ist stark abhängig vom Lösungsmittelgemisch und in der DCE:MeCN-8:2-Mischung am niedrigsten. Beim 1:1-Gemisch nimmt die Intensität der Signale stark zu, da auch die Polarität der Lösungsmittelmischung mit zunehmendem MeCN-Anteil steigt. In der 3:7-Mischung von DCE zu MeCN sinken die Intensitäten der PE-Ketten wieder leicht durch die zunehmende Verdünnung und der sinkenden PE-Konzentration. Die 1:1 Mischung von MeCN und DCE liefert also die höchsten Intensitäten und das höchste S/R Verhältnis. In der 1:1-Mischung sind die Intensitäten von PE-N ${ }^{+}$ mehr als doppelt so hoch wie die der durch Essigsäure protonierten Form von $\mathrm{PE}-\mathrm{NH}^{+}(\mathrm{Abb}$. 3.4 B und C). Die permanente Ammoniumgruppe ist bei der Detektion in der ESI-MS also effektiver, als die Protonierung. Die gefundenen Molmassen stimmen auch in der ESI-MS gut mit den berechneten Werten überein (Tabelle 3.1). Ebenso stimmt das experimentelle Isotopenmuster mit dem theoretischen überein, auch wenn Abweichungen bei dem $1 \mathrm{x}^{13} \mathrm{C}$ und $2 \mathrm{x}^{13} \mathrm{C}$-Isotopen auftreten. Das Peakmaximum ist von $\mathrm{PE}-\mathrm{N}^{+}$gegenüber $\mathrm{PE}-\mathrm{NH}^{+}$ um $28 \mathrm{~g} \mathrm{~mol}^{-1}$ verschoben, was genau der Differenz der Endgruppen beider Polymere 

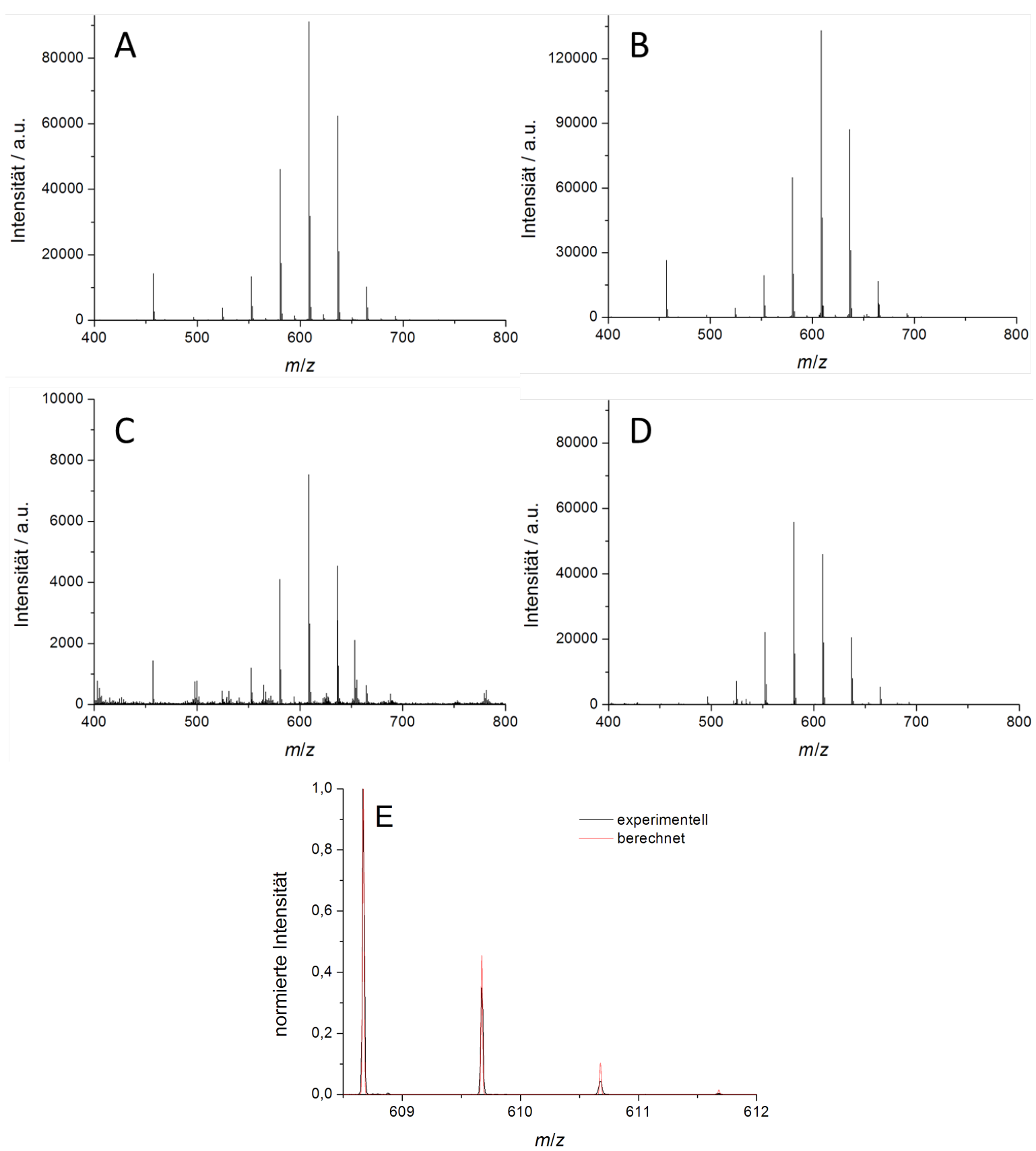

Abb. 3.4. Die ESI-Spektren von PE-N ${ }^{+}$bei einem Lösungsmittelverhältnis von MeCN zu DCE von 7:3 (A), 1:1 (B) und 2:8 (C) sowie das Spektrum von PE-NH ${ }^{+}$in MeCN zu DCE von 1:1 (D). Abbildung $\mathrm{E}$ zeigt das theoretische und experimentelle Isotopenmuster. 
entspricht. Im Vergleich zur MALDI-MS ist die Anzahl der detektierbaren, unterschiedlichen Ketten geringer. Dies ist vermutlich auf das Filtrieren der nach dem Abkühlen entstandenen Suspension zurückzuführen. Es fallen bevorzugt die längeren Ketten aus, die dann durch Filtration entfernt werden. Des Weiteren ist es möglich, dass die oben erwähnten Massendiskriminierungseffekte bei dem ESI-Massenspektrometer stärker ausgeprägt sind. 158

Zusammenfassend wurde in diesem Abschnitt anhand von NMR-Spektroskopie gezeigt, dass die nucleophile Substitution des lods mit einem sekundären oder tertiären Amin quantitativ verläuft. Die polaren Endgruppen erlauben auch eine massenspektromtrische Analyse von PE, sodass die Endgruppe exakt bestimmt und die Molmasse von PE zumindest abgeschätzt werden kann.

\subsection{Phasentransfereigenschaften und Beladung von PE mit anionischen, organischen Verbindungen}

Über die massenspektrometrische Analyse hinaus bietet die kationische Endgruppe weitere Anwendungsmöglichkeiten, die im Folgenden anhand von PE- ${ }^{+}$demonstriert werden. Die erste Anwendung betrifft die Phasentransfereigenschaften des geladenen Polymers, die in der Literatur bereits für andere Polymersorten beschrieben wurden. ${ }^{[153[154]}$ Dazu wurde eine wässrige Lösung des anionischen Farbstoffs Methylorange (MO) einmal mit Toluol (Blindprobe) und einmal mit einer $90^{\circ} \mathrm{C}$ heißen Lösung von PE- $\mathrm{N}^{+}$in Toluol überschichtet. Damit das Polymer nicht durch Abkühlen ausfällt, wurde diese Mischung weiterhin auf $90^{\circ} \mathrm{C}$ erwärmt. Zuerst befindet sich der gelb bis orangene Farbstoff aufgrund seines ionischen Charakters in beiden Gefäßen in der unteren, wässrigen Phase. Nach dem Schütteln beider Proben verweilt das $\mathrm{MO}$ in der Blindprobe weiterhin in der wässrigen Phase, wohingegen bei der PE-N+-Lösung der Farbstoff vollständig in die organische Phase übergeht (Abb. 3.5 links und rechts). Die Triebkraft für den Anionenaustausch von lodid (Gegenion von PE- $\mathrm{N}^{+}$) und dem anionischen MO kann der starken Solvatisierung des in der wässrigen Phase ent-

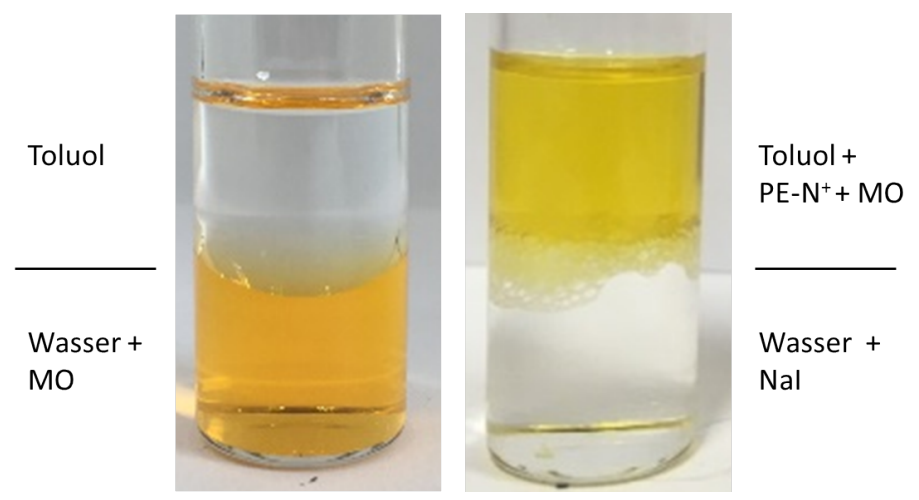

Abb. 3.5. Links: ein Zwei-Phasensystem nach Schütteln bestehend aus Toluol und MO gelöst in Wasser. Rechts: ein Zwei-Phasensystem nach Schütteln bestehend aus PE-N ${ }^{+}$und $\mathrm{MO}$ gelöst in Toluol sowie Wasser. 


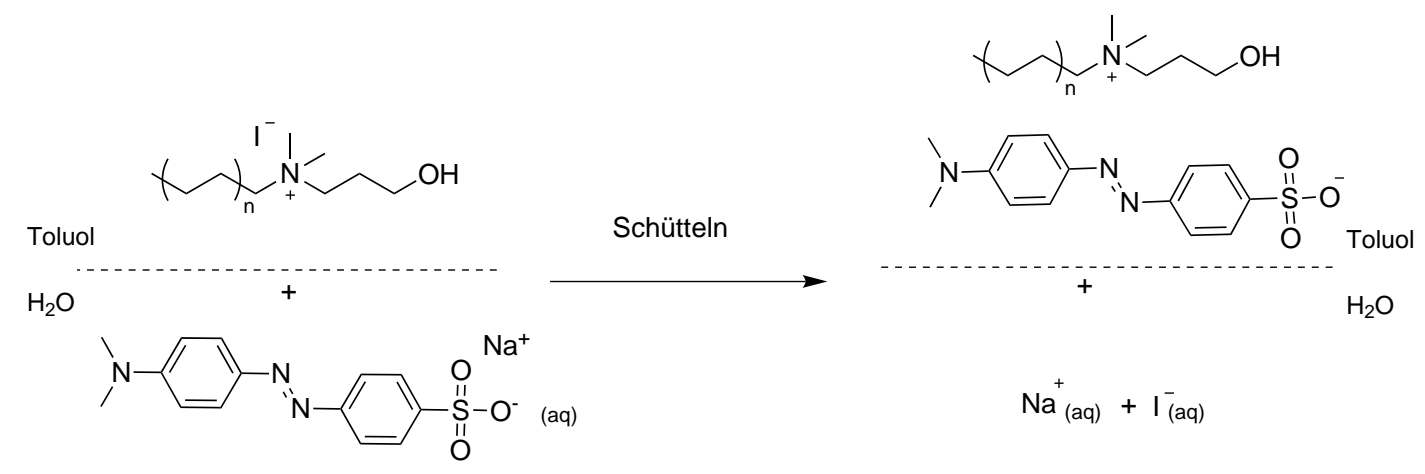

Schema 3.2. Stattfindener lonenaustausch während des Schüttelns der rechten Lösung aus Abb. 3.5.

stehenden $\mathrm{Nal}$ zugeordnet werden (Schema 3.2). Zudem ist anzunehmen, dass das MO aufgrund seiner organischen Struktur hydrophober als lodid ist und daher die organische Phase bevorzugt.

Für potentielle Anwendungen und um die Stärke der ionischen Wechselwirkungen zwischen $\mathrm{MO}$ und $\mathrm{PE}-\mathrm{N}^{+}$zu überprüfen, wurde die gelbe und Polymer-enthaltende organische Phase (Abb. 3.5 rechts) abpipettiert. Das Lösungsmittel wurde im Vakuum entfernt und so ein orange-gefärbtes PE (PE-N ${ }^{+}-\mathrm{MO}^{-}$; Abb. 3.6A) erhalten. Zu Vergleichszwecken wurde ein kommerziell erhaltenes PE ohne ionische Endgruppe analog zu PE-N ${ }^{+}-\mathrm{MO}^{-}$behandelt. Dabei wurde zusätzlich das kommerzielle Phasentransferreagenz Tetrabutylammoniumbromid (TBAB) eingesetzt, damit ein Teil des MO nach dem Schütteln in die Toluolphase übergeht. Erneut wurde die organische Phase abpippetiert und das Lösungsmittel im Vakuum entfernt, um gelb gefärbtes PE (MO@PE; Abb. 3.6B) zu erhalten. Beide eingefärbte PEs (PE$\mathrm{N}^{+}-\mathrm{MO}^{-}$und MO@PE) wurden als Feststoffe mit mehreren Lösungsmittel (Wasser, Ethanol und THF) behandelt. Dabei bleicht MO@PE schnell aus, da der Farbstoff nur mit ausgefällt und - wenn überhaupt - nur schwach am PE gebunden ist (Abb. 3.6P). PE- $\mathrm{N}^{+}-\mathrm{MO}^{-}$weist eine exzellente Stabilität gegenüber den verwendeten Lösungsmitteln auf und bleicht nicht aus (Abb. 3.6C). Der anionische Farbstoff ist über die starken ionischen Wechselwirkungen fest an das PE-N $\mathrm{N}^{+}$gebunden. PE mit terminaler, protonierter Amingruppe wurde ebenfalls auf seine Phasentransfereigenschaften hin untersucht. Dazu wurde das obige Beladungsexperiment von $\mathrm{PE}-\mathrm{N}^{+}$mit PE- $\mathrm{NH}^{+}$wiederholt. Es stellte sich jedoch heraus, dass $\mathrm{MO}$ nicht in die organische Phase überging. Diese Beobachtung zeigt eindeutig, dass die Phasentransfereigenschaften des permanent geladenen $\mathrm{PE}-\mathrm{N}^{+}$wesentlich besser sind, als die der protonierten Variante PE-NH+.

Es konnte gezeigt werden, dass anionische Verbindungen ohne Additive effektiv und dauerhaft über eine Endgruppenmodifizierung in unpolares PE eingebaut werden können. Der anionische Farbstoff MO diente hier als Modellsystem für den stabilen Einbau von Verbindungen in PE über ionische Wechselwirkungen. Diese Einbaumethode könnte in Zukunft auch auf andere anionische Verbindungen wie z.B. Antibiotika in antibakteriellen Lebensmittelverpackungen oder nicht flüchtigen Weichmachern und Flammschutzmittel übertragen werden. 159/160] 

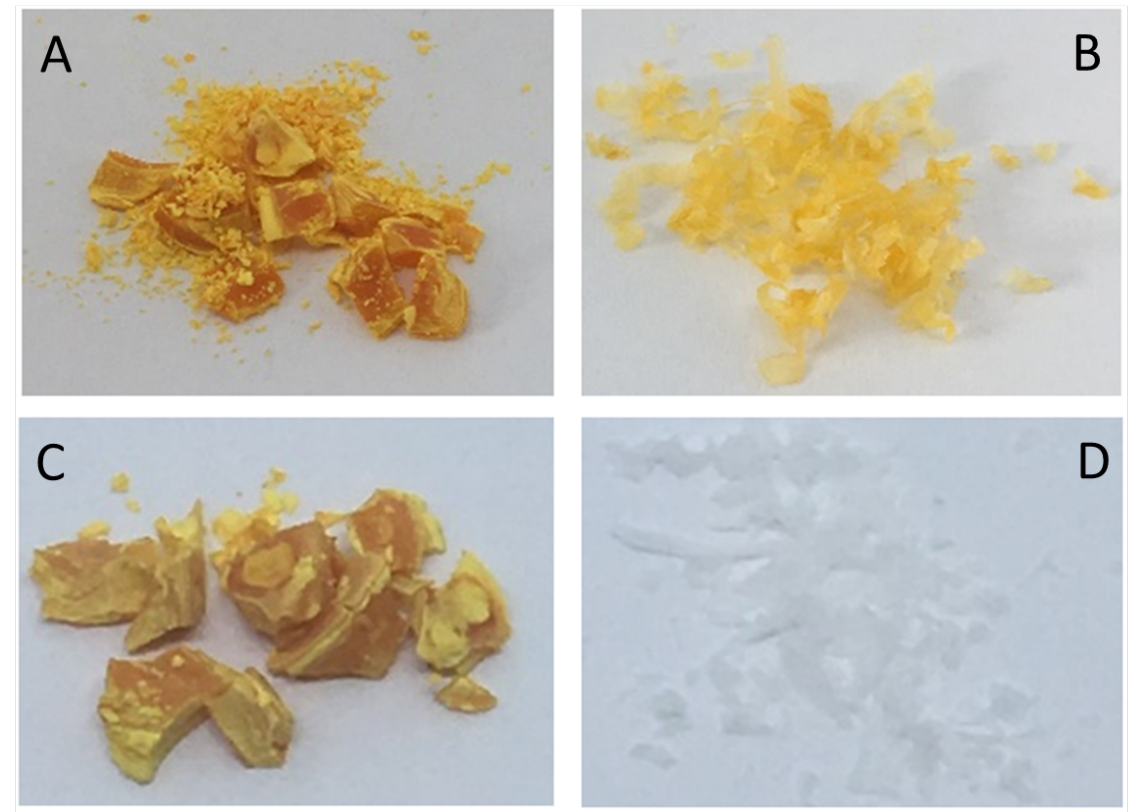

Abb. 3.6. Mit dem anionischen $M O$ beladenes $P E$ vor den Extraktionsexperimenten [oben: PE$\mathrm{N}^{+}-\mathrm{MO}^{-}(\mathrm{A})$ und $\left.\mathrm{MO} @ \mathrm{PE}(\mathrm{B})\right]$ und danach [unten: PE-N+ $\mathrm{N}^{+} \mathrm{MO}^{-}(\mathrm{C})$ und $\left.\mathrm{MO} @ \mathrm{PE}(\mathrm{D})\right]$.

\subsection{Verwendung von kationischem PE als Phasentransferkatalysator}

Des Weiteren wurde der Einsatz von PE-N ${ }^{+}$als PTK getestet. Dieser ist in der Literatur bereits durch PE mit kationischer Endgruppe, synthetisiert über die anionische Polymerisation oder durch Modifizierung von oxidiertem PE, untersucht worden. [104|105] In dieser Arbeit liegt der Fokus besonders auf dem direkten Vergleich des PE- $\mathrm{N}^{+}$mit etablierten, kommerziell erhältlichen PTKs. Dazu wurde die nucleophile Substitution von 1-Bromdecan mit Natriumcyanid im Zweiphasensystem Wasser / $n$-Octan als Modellreaktion bei $100-110{ }^{\circ} \mathrm{C}$ untersucht (Schema 3.3). Zum Vergleich wurden TBAB, Tetrabutylammoniumiodid (TBAI), Tetraphenylphosphoniumbromid (TPPB) und Tetraoctylammoniumbromid (TOAB) ebenfalls in der Modellreaktion als PTK eingesetzt (Schema 3.4). In der Modellreaktion wurden für die unterschiedlichen PTKs die Bedingungen wie Temperatur und Konzentrationen von $\mathrm{NaCN}$, Bromdecan sowie des PTKs gleich gehalten. Der PTK wurde immer in einer Konzentration von 2 mol\% bezogen auf Bromdecan zugegeben. Bei PE- $\mathrm{N}^{+}$wurde für die Berechnung der Konzentration der $M_{\mathrm{n}}$ aus der GPC verwendet. Allerdings wurde die Endgruppenfunktionali-

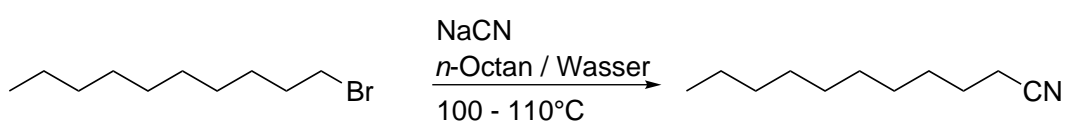

Schema 3.3. Die Modellreaktion zur Untersuchung der PTK-Eigenschaften von PE-N+ im Vergleich zu kommerziellen PTKs. 


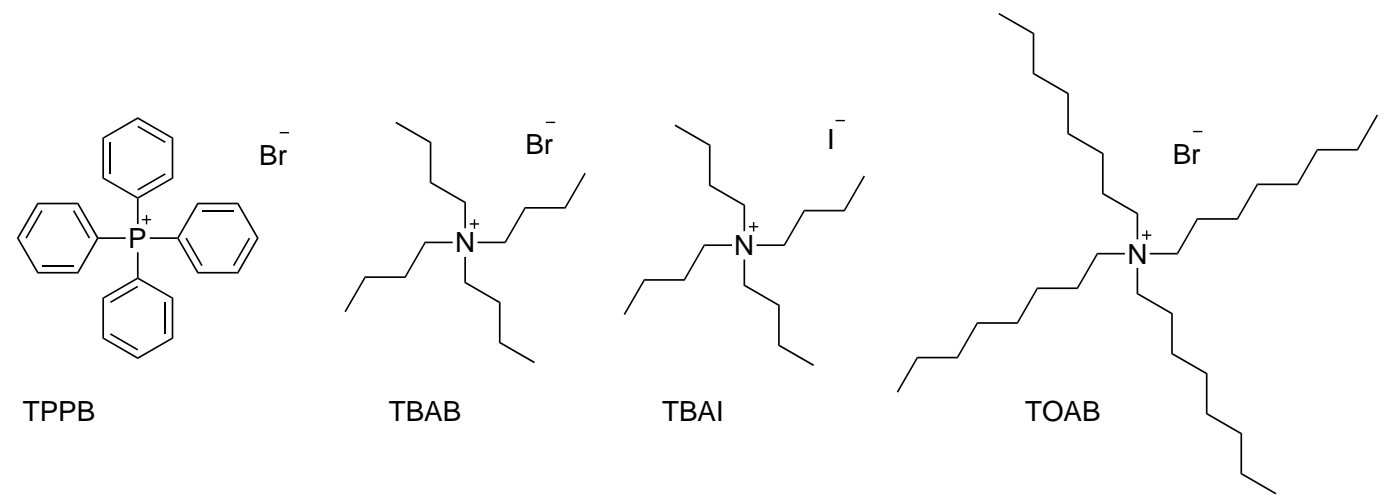

Schema 3.4. Strukturen der hier verwendeten, kommerziellen PTK. Die Struktur von PE-N ${ }^{+}$ wurde bereits in den Schemata 3.2 und 3.1 gezeigt.

tät von nur $83 \%$ nicht mit eingerechnet, sodass die Konzentration von PE- $\mathrm{N}^{+}$entsprechend niedriger als 2 mol\% ist. Zusätzlich wurde eine Blindprobe ohne Einsatz eines PTKs durchgeführt. Tabelle 3.2 zeigt die nach vier oder 18 Stunden mit den unterschiedlichen PTKs erzielten Umsätze von Bromdecan zu Cyanodecan. Die Umsätze wurden mittels ${ }^{1} \mathrm{H}-\mathrm{NMR}$ Spektroskopie und Vergleich der Signale der Methylengruppen $\mathrm{CH}_{2}-\mathrm{Br}$ und $\mathrm{CH}_{2}-\mathrm{CN}$ bei $\delta=3,38$ und 2,31 ppm bestimmt. Ein ausgewertetes Beispiel-NMR-Spektrum findet sich im Anhang A2.

Die Ergebnisse zeigen, dass innerhalb von 4 Stunden die Reaktion ohne PTK und mit TPPB nicht stattfindet, wohingegen PE-N ${ }^{+}$zu einem Umsatz $U$ von $20 \%$ führt. Aus diesem Grund wurden mit TBAB, TBAI und TOAB sterisch weniger anspruchsvolle PTKs mit aliphatischen statt mit polareren aromatischen Seitenketten verwendet. Die entspricht auch eher der Struktur des PE-N ${ }^{+}$. TBAB erzielt nach 18 Stunden weniger als $2 \%$ Umsatz des Bromdecans während PE- $\mathrm{N}^{+}$einen Umsatz von $80 \%$ in der gleichen Zeit erzielt. Es ist bekannt, dass das lodid-Gegenion in $\mathrm{PE}-\mathrm{N}^{+}$, manche nucleophile Substitutionen beschleunigen können, da es als gutes Nucleophil und Abgangsgruppe fungiert. Daher wurde TBAI ebenfalls als PTK getestet. Der erzielte Umsatz ist aber ähnlich zu dem des TBAB und somit kann das lodidgegenion bei PE- $\mathrm{N}^{+}$keine Rolle spielen. Eine weitere Ursache für die bes-

Tab. 3.2. Die erzielten Umsätze von Bromdecan mit den verschiedenen PTK ermittelt über ${ }^{1} \mathrm{H}$ NMR-Spektroskopie.

\begin{tabular}{ccc}
\hline PTK & $t / \mathrm{h}$ & $U / \%$ \\
\hline ohne PTK & 4,18 & 0 \\
TPPB & 4 & 0 \\
PE-N & 4 & 20 \\
TBAB & 18 & $<2$ \\
TBAI & 18 & $<2$ \\
TOAB & 18 & 91 \\
PE-N & 18 & 80 \\
\hline
\end{tabular}




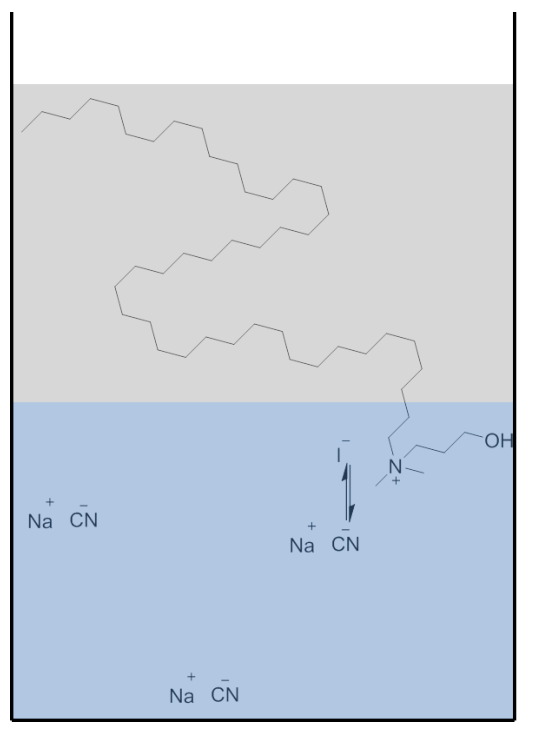

Schema 3.5. Postulierter Mechanismus für den Phasentransfer von Cyanidionen durch PE-N ${ }^{+}$ im Zweiphasensystem von Octan und Wasser. Der polare Kopf der PE-Kette kann zeitweise in die wässrige Phase hineinragen.

sere katalytische Aktivität des polymeren PTKs könnte sein hydrophoberer Charakter sein, da die organische Phase aus dem sehr unpolaren Octan besteht. TBAB, und damit auch das Cyanidion, hält sich hauptsächlich in der wässrigen Phase auf. PE- $\mathrm{N}^{+}$ist hingegen in der wässrigen Phase vollständig unlöslich, hält sich hauptsächlich in der organischen Phase auf. Dadurch hat auch das Gegenion eine höhere Aufenthaltswahrscheinlichkeit in der organischen Phase und die Reaktion wird effizienter katalysiert. Der Phasentransfer des Cyanids kann bei PE-N $\mathrm{N}^{+}$aber trotzdem stattfinden, da die hydrophile Endgruppe entsprechend des postulierten Mechanismus in Schema 3.5 in die wässrige Phase hineinragen kann. Ein hydrophoberer PTK führt demnach im verwendeten System zu einer Beschleunigung der Reaktion.

Tatsächlich konnte bei der Verwendung von TOAB, welche aufgrund seiner längeren Alkylketten wesentlich hydrophober ist als TBAB, der Umsatz auf $91 \%$ gesteigert werden. Dieser Wert ist etwas höher als der des $\mathrm{PE}-\mathrm{N}^{+}(80 \%)$. Allerdings muss berücksichtigt werden, dass nur $83 \%$ der PE-Ketten eine Ammoniumendgruppe tragen. Dadurch weist das hier hergestellte PE- $\mathrm{N}^{+}$in Oktan/Wasser eine höhere Aktivität als TPPB, TBAI und TBAB auf. Die PTK-Aktivität von PE-N ${ }^{+}$ist vergleichbar zu der des TOAB, dem besten hier getesteten kommerziellen PTK. Ein weiterer großer Vorteil von PE mit ionischer Endgruppe ist die Abtrennung des polymeren PTKs. PE- $\mathrm{N}^{+}$ist unterhalb von $80{ }^{\circ} \mathrm{C}$ unlöslich. Es kann daher leicht von dem Produkt der Reaktion durch Filtration abgetrennt werden. 104/105 Die Abtrennung und Aufarbeitung klassischer PTKs ist hingegen aufwendiger. Nach obiger Phasentransferreaktion wurde von dem abfiltrierten PE-N $\mathrm{N}^{+}$erneut ein ${ }^{1} \mathrm{H}-\mathrm{NMR}$-Spektrum aufgenommen. Dieses unterscheidet sich nicht von dem Spektrum vor der Reaktion, sodass das PE-N $\mathrm{N}^{+}$problemlos mehrfach als PTK verwendet werden kann.

In diesem Abschnitt konnte gezeigt werden, dass der PE-basierte PTK aufgrund sei- 
ner hohen Aktivität sowie seiner effizienten und einfachen Rückgewinnung den klassischen PTKs vor allem in sehr hydrophoben organischen Phasen überlegen ist.

\subsection{Weitere Funktionalisierung von PE-N+ und PE-I}

$\mathrm{PE}-\mathrm{N}^{+}$beherbergt an der Ammoniumendgruppe noch eine Hydroxylgruppe. Alle bisher bekannten PEs mit ionischen Endgruppen verfügen über keine weiteren funktionellen Gruppen. $97996-103]$ In diesem Abschnitt wird untersucht, inwiefern die vorhandene Hydroxylgruppe für weitere Reaktionen wie das Einbringen einer Methacrylat-, ATRP-Initiator- oder RAFTAgensfunktion genutzt werden kann. Alle Reaktionen wurden bei Temperaturen oberhalb von $80{ }^{\circ} \mathrm{C}$ durchgeführt, um die PE-Vorstufe in Lösung zu bringen.

\subsubsection{Weitere Funktionalisierungen mittels nucleophiler Substitution}

Ausgehend von PE-I wurde, analog zur Synthese von PE-N ${ }^{+} / \mathrm{PE}-\mathrm{N}$, versucht eine RAFTEndgruppe über eine nucleophile Substitution einzuführen. Dafür wurde PE-I direkt mit dem RAFT-Agens RAFT-Amin, das ein tertiäres Amin enthält, zu PE-N ${ }^{+}-R A F T$ umgesetzt (Schema 3.6. Die Synthese des RAFT-Amins wird in Abschnitt4.2 beschrieben. Dabei wurde die Reaktionszeit und -temperatur sowie das Lösungsmittel - Toluol und verschiedene ToluolDMF-Mischungen - variiert. In dieser Arbeit wird nur die Synthese mit den optimierten Reaktionsbedingungen mit einer Reaktionstemperatur von $90^{\circ} \mathrm{C}$, einer Reaktionszeit von zwei Tagen und einem Lösungsmittelgemsich Toluol:DMF von 9:1 diskutiert.

Abbildung 3.7 zeigt das MALDI-Massenspektrum des Reaktionsproduktes PE-N+RAFT. Die gemessenen $\mathrm{m} / \mathrm{z}$-Werte entsprechen der theoretischen Masse des gewünschten Reaktionsproduktes (siehe Innenbild Abb. 3.7). Das MALDI-Spektrum bestätigt die Entstehung des Produktes. Es kann aber nicht zur quantitativen Analyse verwendet werden, da mögliche Nebenprodukte keine Ammoniumgruppe enthalten und daher mittels MALDI-MS nicht detektiert werden können. Für die quantitative Analyse wurde daher die ${ }^{1} \mathrm{H}$-NMR-Spektroskopie verwendet. Das ${ }^{1} \mathrm{H}$-NMR-Spektrum des Produktes zeigt

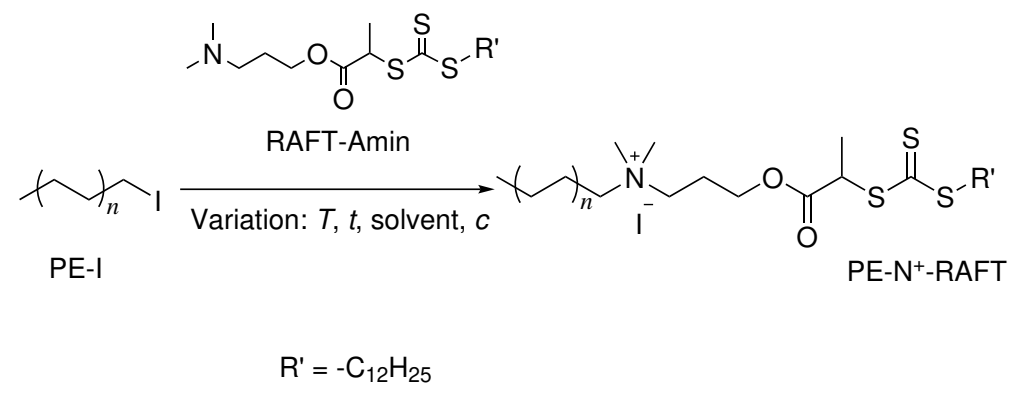

Schema 3.6. Reaktionen von PE-I mit tertiären Aminen. 


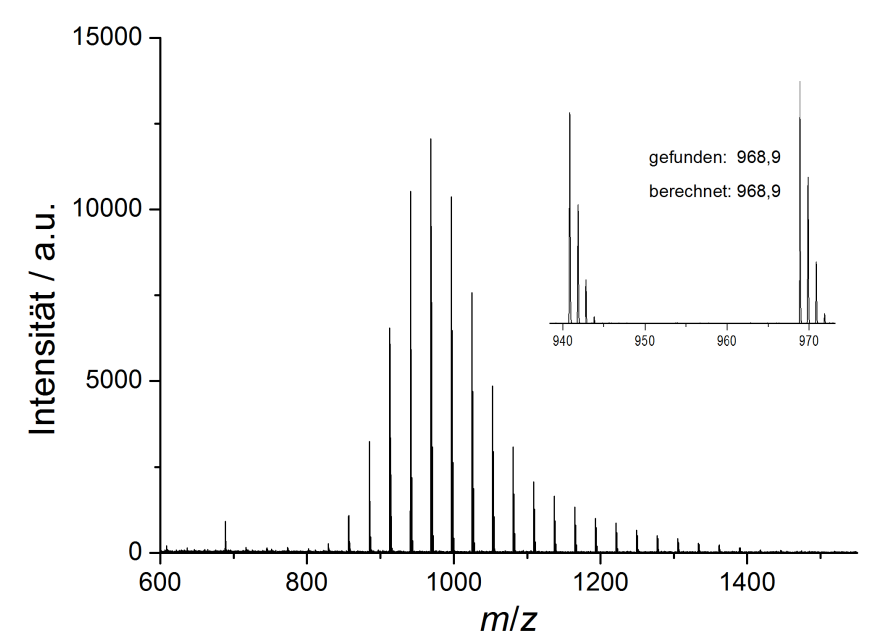

Abb. 3.7. MALDI-Massenspektrum von PE-N+ ${ }^{+}$RAFT synthetisiert entsprechend Schema 3.6

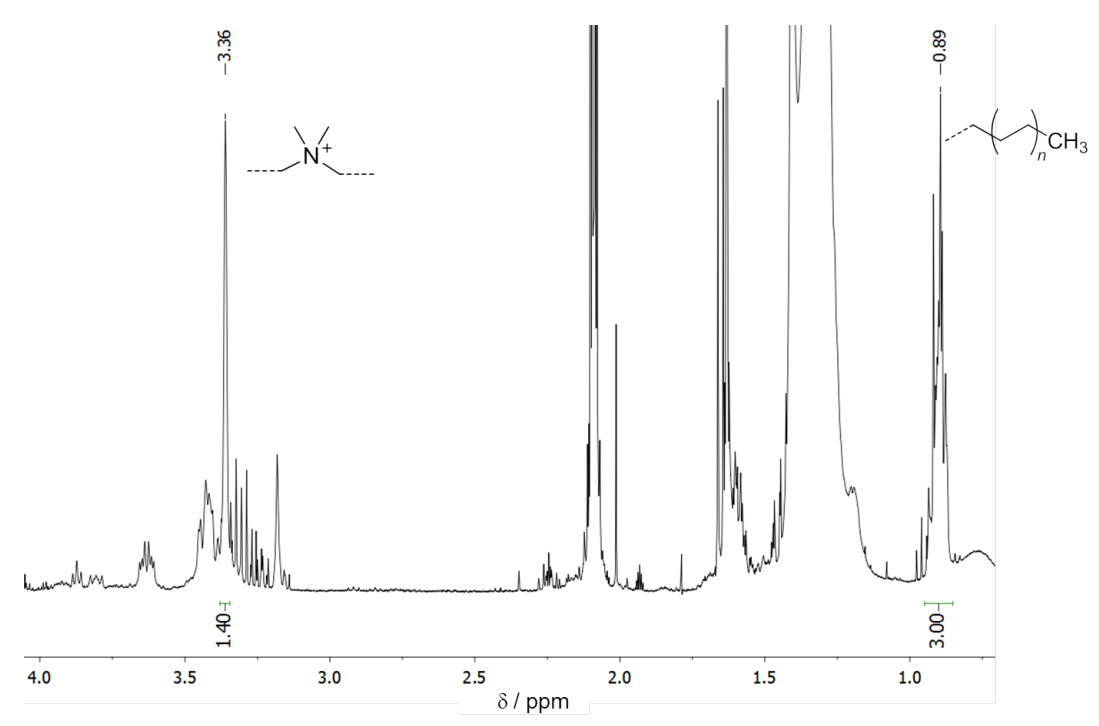

Abb. 3.8. ${ }^{1} \mathrm{H}-\mathrm{NMR}-\mathrm{Spektrum}$ von PE-N ${ }^{+}-\mathrm{RAFT}$ synthetisiert entsprechend Schema 3.6 .

jedoch anhand des Tripletts der endständigen Methylendgruppe bei $\delta=0,89$ ppm und des Singuletts der Methylgruppen am quartären Stickstoff bei $\delta=3,36 \mathrm{ppm}$, dass der Anteil an PE-N ${ }^{+}$-RAFT maximal 20 bis $25 \%$ im isolierten Produkt beträgt (Abb. 3.8). Das Edukt (PE-I) wurde aber vollständig verbraucht, da das Triplett der Methylengruppe neben dem lod verschwunden ist (vgl. dazu das NMR-Spektrum von PE-I, Abb. 3.2). Im NMR-Spektrum des Produktes sind noch weitere, kleinere Signale von etwaigen Nebenprodukten zu erkennen. Diese Nebenprodukte konnten allerdings nicht identifiziert werden. Aufgrund der Nebenprodukte und des nur geringen Anteils von PE-N+2-RAFT, eignet sich dieses Produkt nicht zur Blockcopolymerisation. 


\subsubsection{Weitere Funktionalisierungen durch Veresterung der Hydroxylgruppe}

\section{Veresterung mit einem RAFT-Agens}

Auch die direkte Veresterung der Hydroxylgruppe des PE-N ${ }^{+}$mit der Carboxylgruppe des RAFT-Agens RAFT-COOH, dessen Synthese in Abschnitt 4.2 besprochen wird, wurde untersucht (Schema 3.7). Für die Veresterung wurde die Carbonsäure entweder mittels Thionylchlorid oder mittels Dicyclohexylcarbodiimid (DCC) sowie 4-(Dimethylamino)-pyridin (DMAP) als Katalysator aktiviert. Letztere Aktivierung ist auch als Steglichveresterung bekannt. In beiden Fällen wurden die Reaktionsbedingungen wie die Temperatur, die Reaktionszeit, das Lösungsmittel und die Konzentration der Edukte variiert.

Die Umsetzung des RAFT-COOH zum entsprechenden Säurechlorid ergab ein tiefschwarzes Produkt. Die nachfolgendeS Reaktion dieses Säurechlorides mit PE- $\mathrm{N}^{+}$führte nicht zum gewünschten PE-N ${ }^{+}$-RAFT. Möglicherweise ist bei der Herstellung der Säurechlorids bereits das RAFT-Agens degradiert. Der Autor dieser Arbeit stellte auch in anderen Projekten fest, dass die Herstellung eines Säurechlorides aus einem RAFT-Agens Probleme verursacht. Daher wurde versucht das PE-N ${ }^{+}$mittels Steglichveresterung mit dem RAFT-COOH zu verestern. Das ${ }^{1} \mathrm{H}-\mathrm{NMR}$-Spektrum des Produktes aus dieser Steglichveresterung ist in Abbildung 3.9 gezeigt. Auch hier entstand nicht das gewünschte PE- $\mathrm{N}^{+}-\mathrm{RAFT}$. Das charakteristische Singulett des Eduktes und des zu erwartenden Produktes bei $\delta=3,4$ ppm fehlt. Es konnte auch kein MALDI-Spektrum des Produktes erhalten werden. Es wurde vermutet, dass eventuell die Ammoniumgruppe bei der Veresterung von PE- $\mathrm{N}^{+}$stört. Daher wurde PE- ${ }^{+}{ }_{6}$ synthetisiert, bei dem die Alkylgruppe zwischen Ammonium- und Hydroxylgruppe verlängert wurde. Die Synthese und Charakterisierung von $\mathrm{PE}-\mathrm{N}^{+}{ }_{6}$ wurde bereits in $\mathrm{Ab}$ schnitt 3.2 besprochen. Um einen Einfluss der Ammoniumgruppe komplett auszuschließen, wurden die Veresterungen auch mit PE-N durchgeführt. Allerdings lieferten beide Veresterungsreaktionen sowohl bei PE- $\mathrm{N}^{+}{ }_{6}$ als auch bei PE-N ähnliche Ergebnisse wie bei PE-N $\mathrm{N}^{+}$. Eine Veresterung war nicht möglich. Daraus folgt vermutlich, dass sich die Ammonium- oder Aminendgruppen des Eduktes im Laufe der Reaktion zersetzen, da auch die charakteristi-
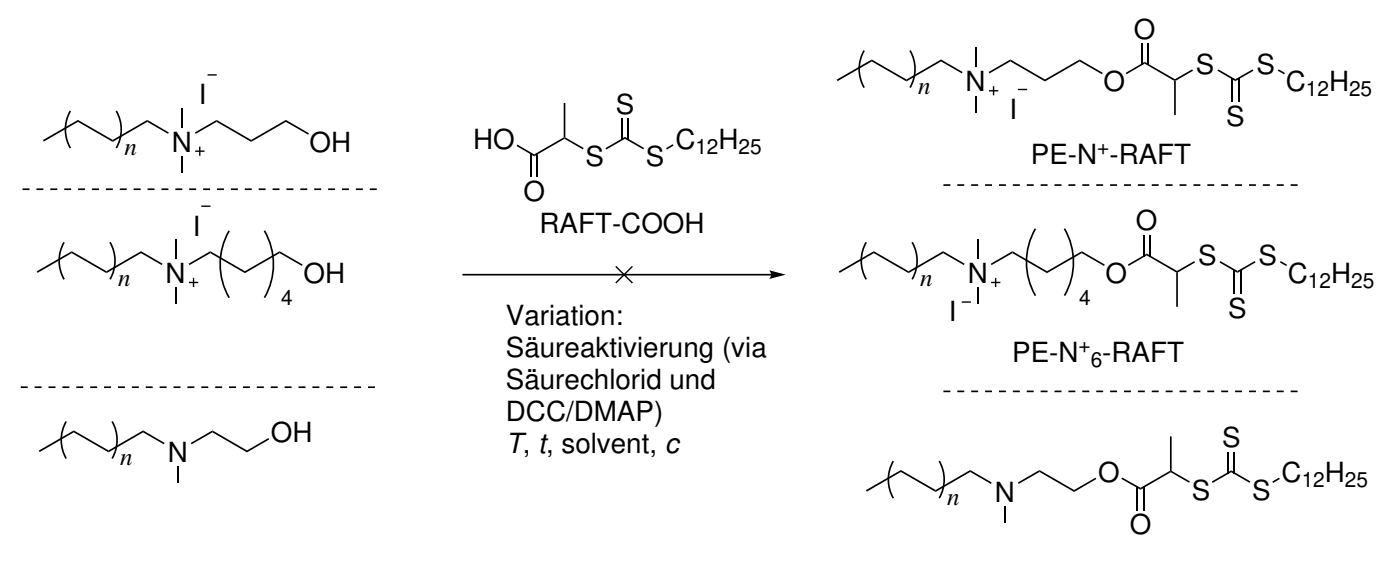

PE-N-RAFT

Schema 3.7. Reaktionen zur Veresterung von PE-N $\mathrm{N}^{+}, \mathrm{PE}-\mathrm{N}^{+}{ }_{6}$ und PE-N. 
schen Eduktsignale im NMR-Spektrum fehlen.

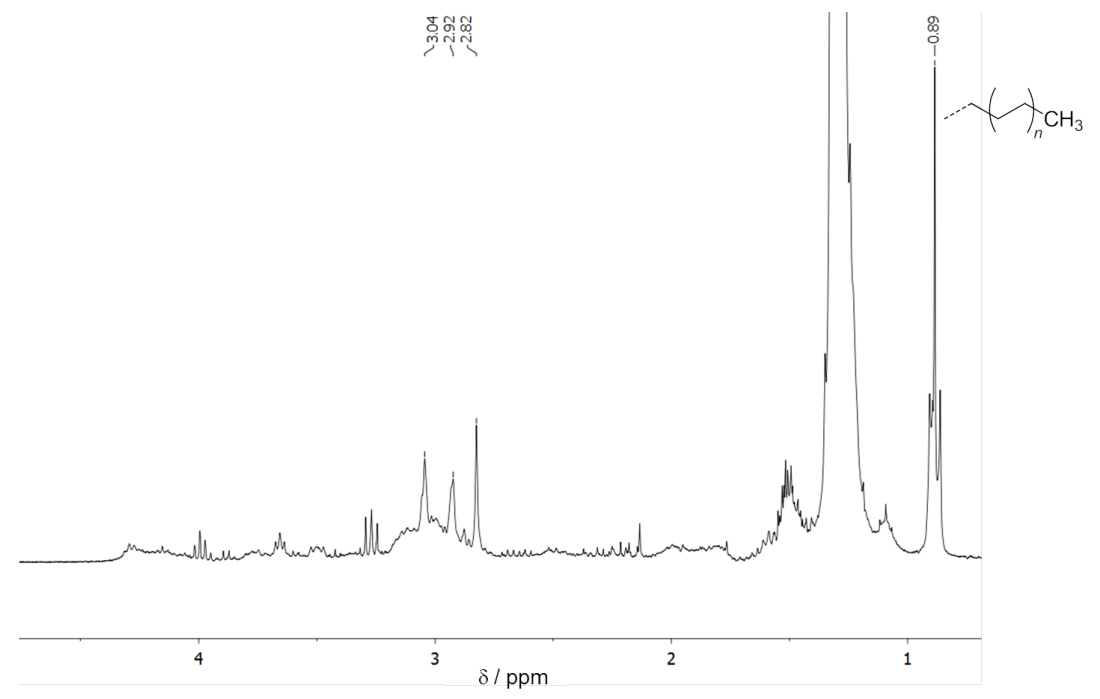

Abb. 3.9. ${ }^{1} \mathrm{H}-\mathrm{NMR}-\mathrm{Spektrum}$ des Produktes aus der Steglichveresterung von $\mathrm{PE}-\mathrm{N}^{+}$mit dem RAFT-Agens RAFT-COOH entsprechend Schema 3.7. Das NMR-Spektrum wurde in einer Mischung aus deuteriertem Benzol und Tetrachlorethan aufgenommen.

\section{Veresterung von PE- $\mathrm{N}^{+}$mittels Carbonsäurehalogeniden}

Die zuvor beschriebenen Modifizierungen von PE-N $\mathrm{N}^{+}$führten lediglich mit sehr geringen Ausbeuten zu dem gewünschten RAFT-modifizierten PE und wiesen zusätzlich Nebenreaktionen auf. Weitaus vielversprechender zeigten sich erste Experimente zur Einführung eines ATRP-Initiators bzw. einer Methacrylatfunktionalität.

Entsprechend Schema 3.8 wurde $\mathrm{PE}^{-N^{+}}$mit dem kommerziell erhältlichen 2Brompropionsäurebromid oder Methacrylsäurechlorid zu PE- $\mathrm{N}^{+}$-ATRP oder zu PE-N $\mathrm{N}^{+}-\mathrm{MA}$ verestert. Abbildung 3.10 zeigt die ${ }^{1} \mathrm{H}$-NMR-Spektren der isolierten Produkte und bestätigt die erfolgreiche Synthese. Bei PE-N+-ATRP (rotes Spektrum in Abb. 3.10) treten die charakteristischen Signale ,wie das Singulett der Methylgruppen am Stickstoff bei $\delta=3,4 \mathrm{ppm}$, das Quartett der $\mathrm{CH}$-Gruppe neben dem Ester bei $\delta=4,7$ ppm sowie das Dublett der Methylgruppe neben dem $\mathrm{CH}$ bei $\delta=1,8 \mathrm{ppm}$, auf. Die Bestimmung der Integrale lässt jedoch vermuten,

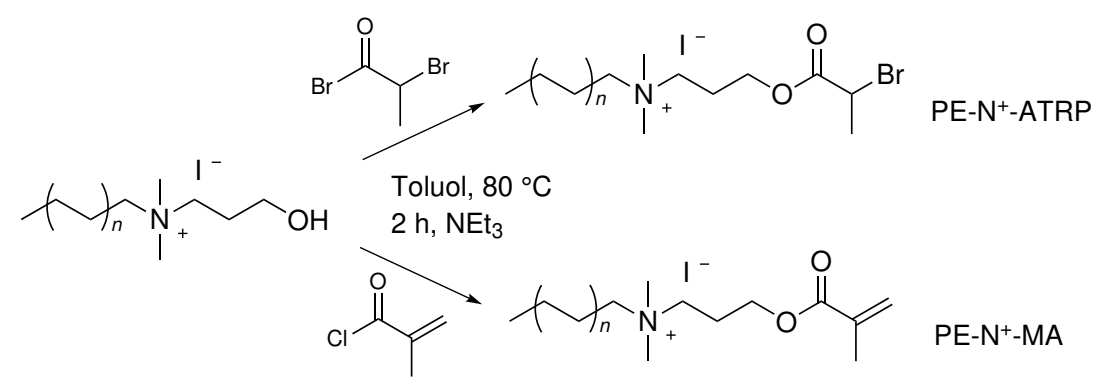

Schema 3.8. Erfolgreiche Modifikationen von PE- $\mathrm{N}^{+}$und PE-N ${ }_{6}{ }_{6}$. 


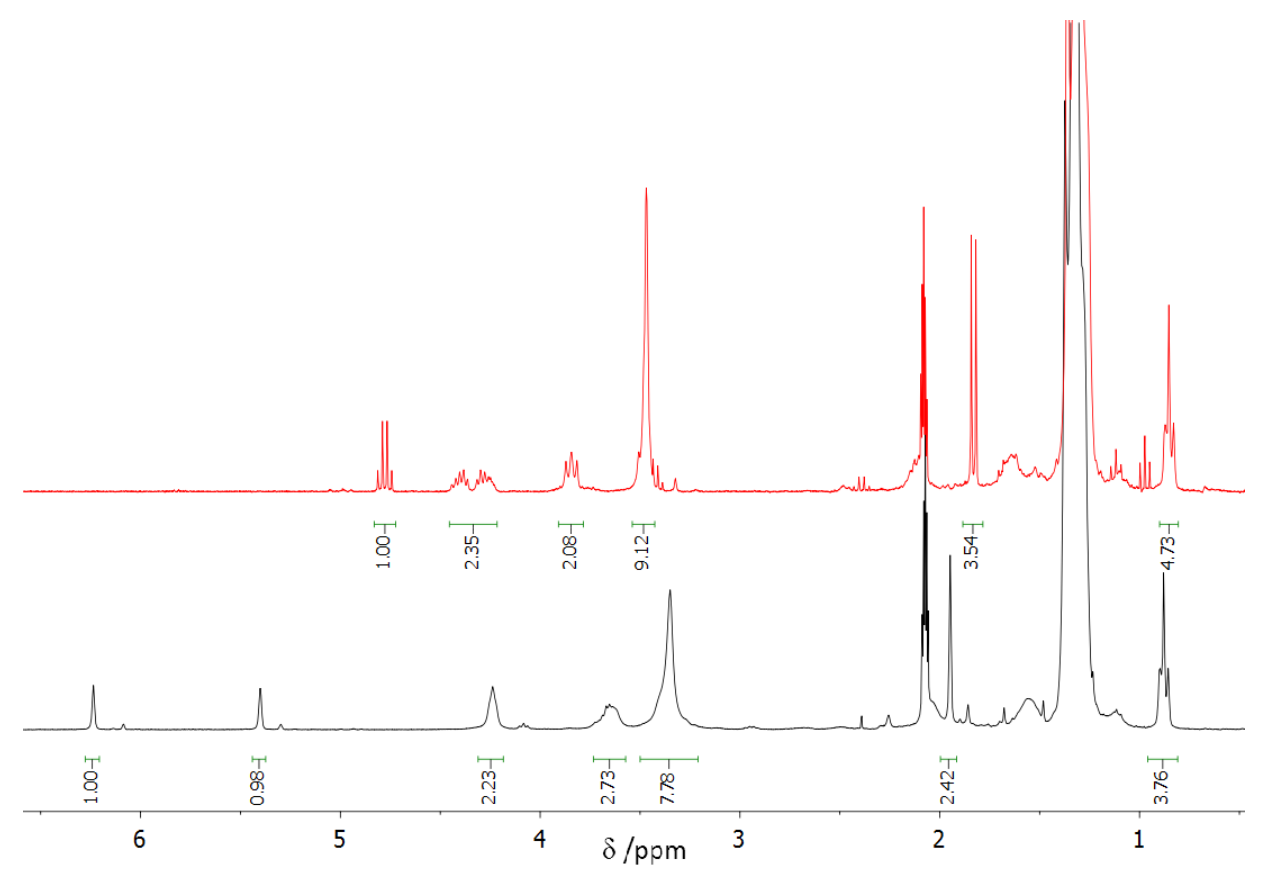

Abb. 3.10. ${ }^{1} \mathrm{H}-\mathrm{NMR}-\mathrm{Spektrum}$ des Produktes aus der Veresterung von PE-N ${ }^{+}$mit Methacrylsäurechlorid (schwarz) und 2-Bromopropionsäurebromid (rot).

dass das erhaltene Produkt nicht rein ist und es während der Reaktion zu Nebenprodukten kommt. Dieser Anteil an Verunreinigung ist aber im Vergleich zu den zuvor besprochenen Syntheserouten sehr gering. Ebenfalls war die Reaktion von PE- $\mathrm{N}^{+}$mit Methacrylsäurechlorid erfolgreich und das Produkt konnte mittels ${ }^{1} \mathrm{H}$-NMR-Spektroskopie bestätigt werden. Die Protonen der endständigen Doppelbindung bei $\delta=5,4$ und 6,2 ppm, der Methylgruppen am quartären Stickstoff bei $\delta=3,3 \mathrm{ppm}$ und der Methylgruppe an der Doppelbindung bei $\delta=1,9$ ppm können eindeutig zugewiesen werden. Jedoch zeigt der Vergleich der Integrale erneut einen geringen Anteil an Nebenprodukten.

Die MALDI-Massenspektren (Abb. 3.11A und B) bekräftigen die Untersuchung der NMRSpektroskopie. Der Abstand zwischen den einzelnen Ketten beträgt $28 \mathrm{~g} \mathrm{~mol}^{-1}$ und bestätigt, dass es sich um PE handelt. Die gefundenen Massen der einzelnen Polymerketten stimmen mit den berechneten überein (Abb. 3.11 und Tabelle 3.1). Das PE- $\mathrm{N}^{+}-$ATRP Spektrum weist, eventuell durch den nicht vollständigen Umsatz und den kleinen Anteil an Nebenprodukten, ein schlechteres S/R-Verhältnis auf. Alle Peaks zeigen ebenfalls das charakteristische Isotopenmuster des Bromatoms (Abb. 3.11 C). Das Spektrum des PE-N+-MA weist eine besseres S/R-Verhältnis auf, zeigt aber eine zweite, kleinere Verteilung mit ein Massendifferenz von +8 gegenüber der Hauptverteilung. Dieser kleineren Verteilung konnte jedoch keiner Endgruppenstruktur zugeordnet werden. 

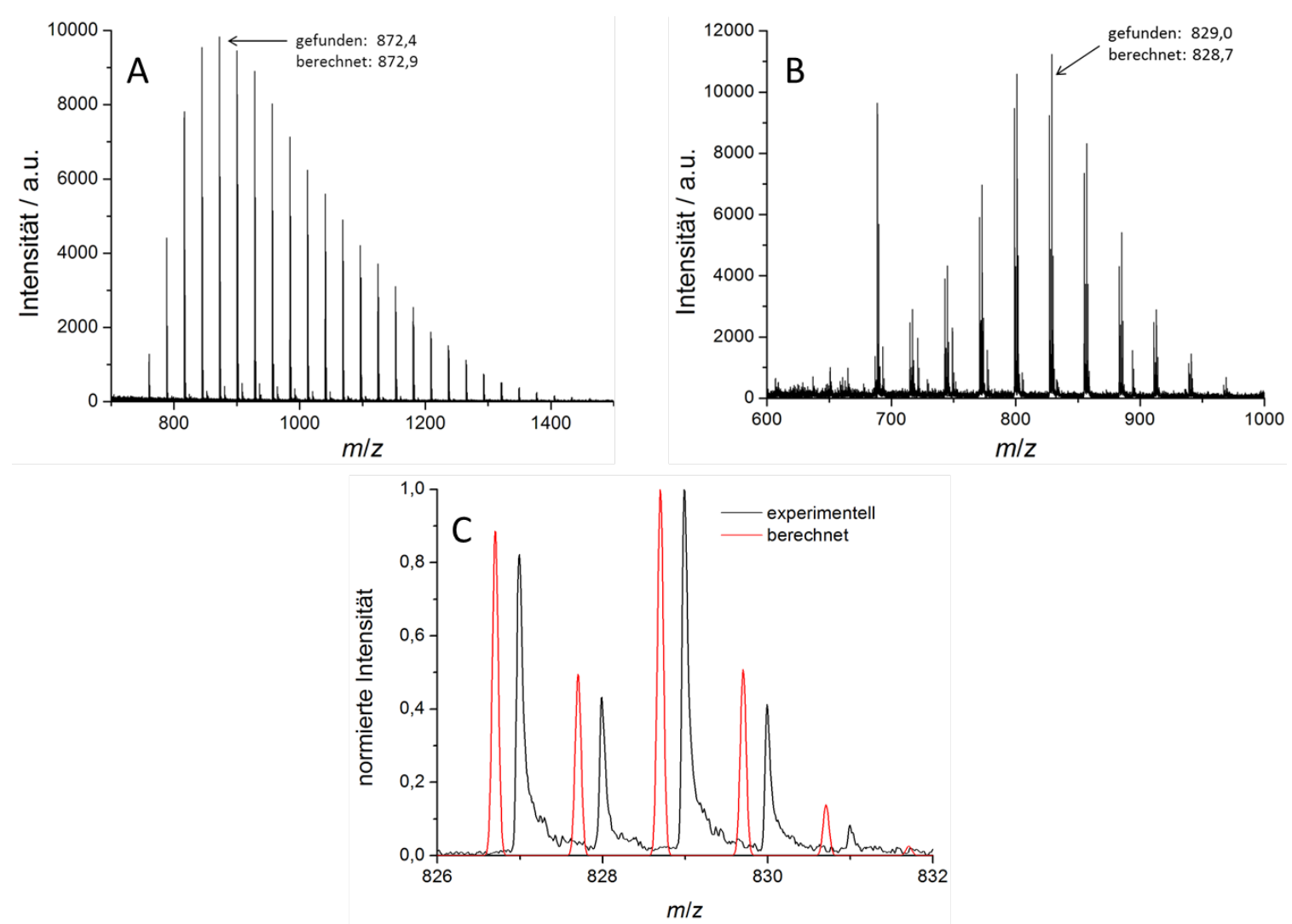

Abb. 3.11. MALDI-Massenspektren des Produktes aus der Veresterung von PE- $\mathrm{N}^{+}$mit Methacrylsäurechlorid (A) oder 2-Bromopropionsäurebromid (B) sowie der Vergleich des berechneten und experimentellen Isotopenmusters von PE- $\mathrm{N}^{+}-A T R P(C)$.

\subsection{Blockcopolymerisation von PE-N+2-ATRP mit Butylacrylat}

Das hergestellte PE-N+-ATRP ist ein ATRP-Makroinitiator und wird in diesem Abschnitt zur Synthese eines Blockcopolymers mittels ATRP eingesetzt. Als Monomer für den zweiten Block wurde $n$-Butylacrylat (BA) verwendet und die Polymerisation erfolgte mit $\mathrm{CuBr}$ und $N, N, N$, N",N"-Pentamethyldiethylentriamin (PMDETA) in Toluol:Anisol bei $100^{\circ} \mathrm{C}$ (Schema 3.9. . Nach der Polymerisation und der Entfernung des Lösungsmittels wurde ein Massenzuwachs beobachtet und ein klebriges Polymer erhalten. Als Blindprobe wurde statt PE- $\mathrm{N}^{+}-$ ATRP nur PE- $\mathrm{N}^{+}$verwendet. In diesem Fall ist also kein ATRP-Initiator vorhanden und es konnte nach der Polymerisation kein Massenzuwachs beobachtet werden. Es ist also weder ein Blockcopolymer noch Polybutylacrylat als Homopolymer entstanden.

Normalerweise wird der Kupferkomplex nach der Polymerisation chromatographisch über Aluminiumoxid abgetrennt. Diese Aufarbeitung war jedoch nicht möglich, da das erhaltene Bockcopolymer aus PE und Polybutylacrylat (PE- $b$-PBA) bei Raumtemperatur ähnlich unlöslich ist wie PE. Der Großteil des Kupferkomplexes konnte aber durch Waschen mit Methanol und verdünnter Salzsäure entfernt werden. Abbildung 3.12 zeigt die bimodale Molmassenverteilung von PE-b-PBA und die Molmassenverteilung des für das Blockcopolymer 


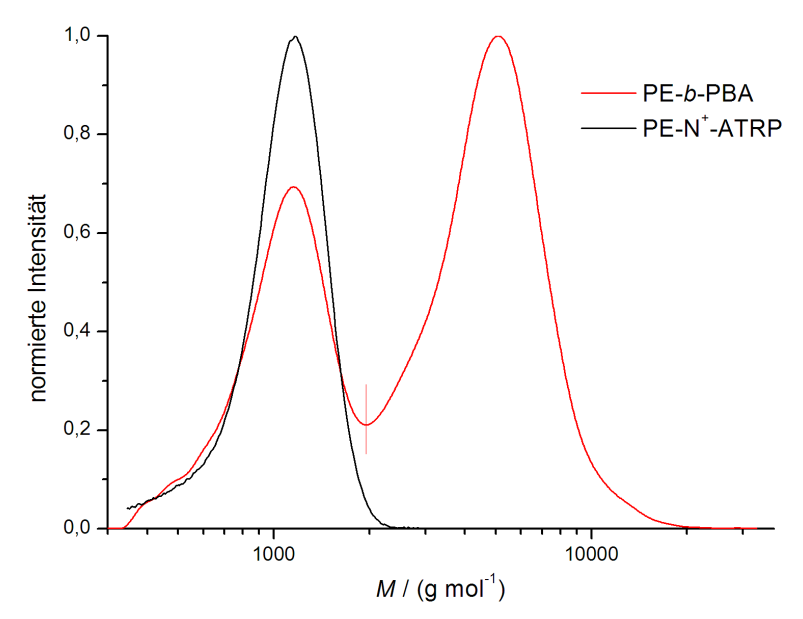

Abb. 3.12. GPC-Chromatogramm des Maktroinitiators PE- $N^{+}-A T R P$ und des PE- $b$-PBA Blockcopolymers (rot).

verwendeten ATRP Makroinitiators. Der Hauptpeak bei etwa $5000 \mathrm{~g} \mathrm{~mol}^{-1}$ resultiert aus dem entstandenen Blockcopolymer. Der Umsatz der Synthese von PE- $\mathrm{N}^{+}$-ATRP war nicht vollständig und es traten Nebenreaktionen auf. Der zweite Peak bei niedrigerer Molmasse gehört daher zu PE-Ketten, die nicht mit dem ATRP-Initiator funktionalisiert sind. Er stimmt mit der MMV des PE-N+-ATRPs überein. Zudem ist es möglich, dass die ATRP-Initiierung nicht bei allen Initiatoren funktionierte. Die zwei Peaks der bimodalen Verteilung überlappen zu einem kleinen Teil. Werden die Peaks dennoch bis zur roten Markierung in der Abbildung 3.12 ausgewertet, liegen die Dispersitäten beider Verteilungen zwischen 1,1 und 1,2, sodass die Polymerisation des Blockcopolymers kontrolliert ist. Mithilfe der MALDI-MS konnte kein Massenspektrum des Blockcopolymers aufgenommen werden. Auch der weiterhin enthaltene erste Block ergibt im MALDI-Massenspektrum kein Signal. Eventuell stören enthaltene Verunreinigungen - wie der Kupferkomplex, der nicht vollständig entfernt werden konnte bei der MALDI-MS.

In diesem Abschnitt wurde in einem ersten Experiment gezeigt, dass eine Blockcopolymerisation mit PE-N ${ }^{+}$-ATRP möglich ist. Bei der Blockcopolymerisation und der Aufreinigung des BCP ist noch weiterere Optimierung nötig.

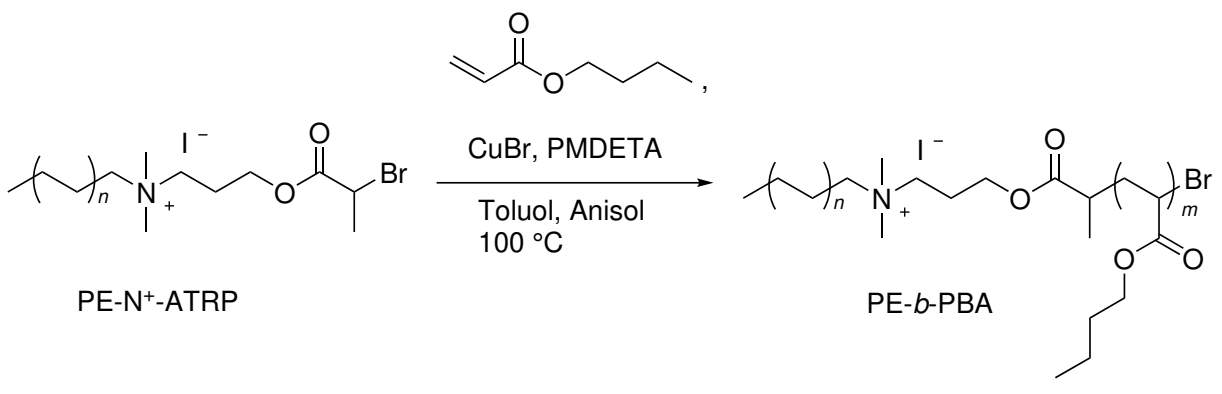

Schema 3.9. Synthese des PE-b-PBA Blockcopolymers über die ATRP. 


\subsection{Schlussfolgerung und Ausblick}

In diesem Kapitel wurde ein neuer Syntheseweg für den Einbau einer kationischen Endgruppe in Polyethylen beschrieben. Dazu wurde PE mittels der CCG Polymerisation hergestellt und durch eine effiziente Reaktion des intermediären metallorganischen PEs eine lodendgruppe erfolgreich eingeführt. Die erhaltene lodendgruppe wurde durch eine quantitative nucleophile Substitution in eine tertiäre Amin- oder Ammoniumgruppe überführt. Beide Endgruppen weisen ein großes Potential in der massenspektrometrischen Analyse von PE mittels MALDI- und ESI-MS auf. Es wurden Spektren mit hohen Signalintensitäten und exzellenten Signal-zu-Rausch-Verhältnissen erhalten.

Die Ammoniumendgruppe ermöglichte den Einbau eines polaren, anionischen Farbstoffes in Polyethylen. Durch die starken ionischen Wechselwirkungen zwischen dem Farbstoff und dem geladenen PE wies das eingefärbte Material eine außerordentliche Lösungsmittelresistenz auf. Der Einbau von anionischen Komponenten über ionische Bindungen könnte auch auf andere Additive wie z.B. Antibiotika, Flammschutzmittel und Weichmacher übertragen werden. Des Weiteren wurde die Verwendung PE- ${ }^{+}$als Phasentransferkatalysator in einer Modellreaktion untersucht. Dabei stellte sich heraus, dass PE- $\mathrm{N}^{+}$effektiver oder genauso effektiv ist wie bereits andere, wohl etablierte Phasentransferkatalysatoren. Darüber hinaus ist der kationische PE-PTK aufgrund seiner stark temperaturabhängigen Löslichkeit leicht von der Reaktion abzutrennen und zudem wiederverwendbar.

Über die vorhandene Hydroxylgruppe wurde PE-N+ durch die Reaktion mit Carbonsäurehalogeniden weiter modifiziert, wodurch ein geladenes PE-Makromonomer und ein geladener PE-ATRP-Initiator erhalten wurde. Beide Polymere konnten erfolgreich via ${ }^{1} \mathrm{H}$-NMRSpektroskopie und MALDI-MS nachgewiesen werden. Mit dem PE-ATRP-Initiator und Butylacrylat wurde zudem erfolgreich eine Blockcopolymerisation durchgeführt. Schlussendlich wurden so die vielfältigen Anwendungsmöglichkeiten von geladenem PE $\left(\mathrm{PE}-\mathrm{N}^{+}\right)$wie die massenspektrometrische Analyse, der Einbau ionischer Verbindungen, die Verwendung als PTK und die Möglichkeit zur weiteren Funktionalisierung einschließlich der Blockcopolymerisation demonstriert.

Zukünftig bedarf die Synthese des PE-ATRP-Initiators und die Blockcopolymerisation noch weiterer Optimierung. Es könnte beispielsweise versucht werden, den ATRP-Initiator mittels Katalyse durch $\mathrm{HfCl}_{4} \cdot 2 \mathrm{THF}$ an PE-N+ anzubringen. 161] Es sollte eine Möglichkeit gefunden werden, eventuelle Verunreinigung wie den Kupferkomplex zu entfernen, da diese möglicherweise in der MALDI-MS stören. Zukünftig könnte durch dynamische Lichtstreuung auch überprüft werden, ob das hergestellte Blockcopolymer PE- $b$-PBA in Lösung mizellare Strukturen ausbildet. Des Weiteren könnte mittels Rasterkraftmikroskopie untersucht werden, ob das Blockcopolymer eine Mikrophasenseparation ausbildet. Aufgrund der stark unterschiedlichen Strukturen von PE und Polybutylacrylat, könnte eine Mikrophasenseparation bereits bei geringen Molmassen erhalten werden. Somit könnte auch die Größe der Phasen bzw. Lamellen reduziert werden, was von großem Interesse für die Herstellung von Nanoschablonen in der mikroelektronischen Industrie ist. 162 


\section{Kationische Endgruppen in RAFT-Polymeren}

Die allgemeine Sensitivität des verwendeten ESI-Massenspektrometers ließ im Laufe dieses Projektes nach, was vermutlich auf ein Problem des Detektors zurückzuführen ist. Daher sind die zu späteren Zeitpunkten durchgeführten Messungen bezüglich der Signalintensität bzw. des S/R-Verhältnisses nicht mehr mit den ersten Messungen vergleichbar.

\subsection{Motivation}

Mit weichen lonisationverfahren wie der ESI- und MALDI-MS können auch Polymere massenspektrometrisch analysiert werden. ${ }^{[15-17]}$ Die Massenspektrometrie wird zur Bestimmung der Molmassenverteilung, der Endgruppen und der Zusammensetzung einzelner (Co)Polymerketten verwendet. 18/20/21/163] Dabei ist die MS wesentlich sensitiver als die GPC, die Infrarot- und die NMR-Spektroskopie. MALDI-Massenspektren weisen jedoch oft ein schlechtes S/R-Verhältnis auf, vor allem wenn eine gute Massenauflösung gefordert ist. Auch in der ESI-MS ist das S/R-Verhältnis für die Analyse von Spezies, die nur in Spuren vorkommen, oft noch zu gering. 164-166 Die ESI-MS beschränkt sich zudem meist auf Polymere geringer Molmassen, da längere Polymere nicht nur einfach sonder auch mehrfach geladene Ketten durch Anlagerungen von Metallkationen bilden können. Da Polymere selbst auch eine Molmassenverteilung aufweisen, entstehen durch die zusätzliche Ladungsverteilung der Ketten komplexe und schwer interpretierbare Massenspektren. 18]19]

Aufgrund der positiven Erfahrungen von ionischen Endgruppen in PE im vorherigen Kapitel ist es naheliegend, den Einfluss dieser Funktionalität in der MS auch anhand weiterer Polymersysteme zu untersuchen. Ziel dieses Projektes ist es daher, PS und PMA unterschiedlicher Molmassen sowohl mit als auch ohne kationische Endgruppen mittels RAFTPolymerisation herzustellen und erstmals systematisch massenspektrometrisch zu analysieren. Dazu wird eine Syntheseroute für neue RAFT-Agenzien mit ein oder zwei Ammoniumgruppen basierend auf Trithiocarbonaten entwickelt. Der Einfluss der eingeführten kationischen Endgruppe auf das S/R-Verhältnis im Vergleich zu den Polymerketten ohne kovalent gebundene ionische Gruppe soll untersucht werden. Es wird vermutet, dass die ionische Endgruppe das S/R-Verhältnis verbessert und so vor allem in der Spurenanalyse von großem Nutzen sein kann. Auch soll festgestellt werden, ob durch den Einbau einer oder zweier ionischer Endgruppen und die dadurch wegfallende Zugabe eines Hilfsalzes eine Reduzierung mehrfach geladener Spezies bzw. eine Kontrolle des Ladungszustandes möglich ist. Dadurch würde die Komplexität der Massenspektren deutlich verringert werden und die ESI-MS könnte auch bei Polymeren höherer Molmasse ihre Anwendung finden. 
Zudem sollen alle auftretenden Spezies der entwickelten RAFT-Polymere sowohl in der MALDI- als auch in der ESI-MS identifiziert werden. Diese vollständige Strukturaufklärung wurde nach besten Wissen des Autors noch nicht für Polymere mit Trithiocarbonatgruppen durchgeführt. Zudem soll der Einfluss verschiedener Mess- und Geräteparameter auf die Signale im Massenspektrum in der ESI-MS untersucht werden, um so die Messbedingungen optimieren zu können.

\subsection{Synthese und Charakterisierung der RAFT-Agenzien}

Der Einbau der kationischen Endgruppen erfolgt bei den RAFT-Polymeren über den Einbau der jeweiligen RAFT-Agenzien, da deren R- und Z-Gruppe die Endgruppen des Polymers bilden (siehe Abschnitt 2.1). Daher mussten zuerst RAFT-Agenzien mit ionischen Endgruppen synthetisiert werden. Das RAFT-Agens RAFT-COOH ist literaturbekannt und dient als Ausgangsmaterial für die Herstellung der ionischen RAFT-Agenzien sowie für die Synthese von RAFT-Polymeren ohne ionische Endgruppen. 167 Die Synthese von RAFT-COOH wurde im Gegensatz zur Literatur leicht modifiziert, um die Aufarbeitung zu erleichtern und die Ausbeute zu steigern. Die hier vorgestellte Synthese von RAFT-COOH ist in Schema 4.1 gezeigt und bedarf keine säulenchromatographische Aufreinigung des Produktes. Im nächsten Schritt wurde RAFT-COOH mit 3-Dimethylamino-1-propanol über eine SteglichVeresterung zum RAFT-Amin verestert. Die Ausbeute beträgt $56 \%$ und ist vergleichbar mit einer ähnlichen Veresterung in der Literatur. ${ }^{168}$ Die Synthese der ionischen RAFTAgenzien ist im Schema 4.2 gezeigt. Das RAFT-Amin wurde entweder mit Methyliodid oder mit 4-(Bromomethyl)benzyltriethylammoniumbromid mit nahezu quantitativer Ausbeute zu RAFT-N ${ }^{+}$oder RAFT-N ${ }^{2+}$ alkyliert. Die Produkte fielen dabei im gewählten Lösungsmittel bereits ohne Verunreinigungen aus. Zur leichteren Handhabung konnte RAFT-N ${ }^{+}$aus Aceton umkristallisiert werden. Das 4-(Bromomethyl)benzyltriethylammoniumbromid wurde entsprechend der Literatur ebenfalls mit nahezu quantitativer Ausbeute isoliert. ${ }^{153]}$ Die Struktur der neuen Verbindungen RAFT-Amin, RAFT-N ${ }^{+}$und RAFT-N ${ }^{2+}$ wurde mittels ${ }^{1} \mathrm{H}-,{ }^{13} \mathrm{C}$ - und 2D-NMR-Spektroskopie sowie Massenspektrometrie bestätigt (Siehe Abschnitt 6.3).

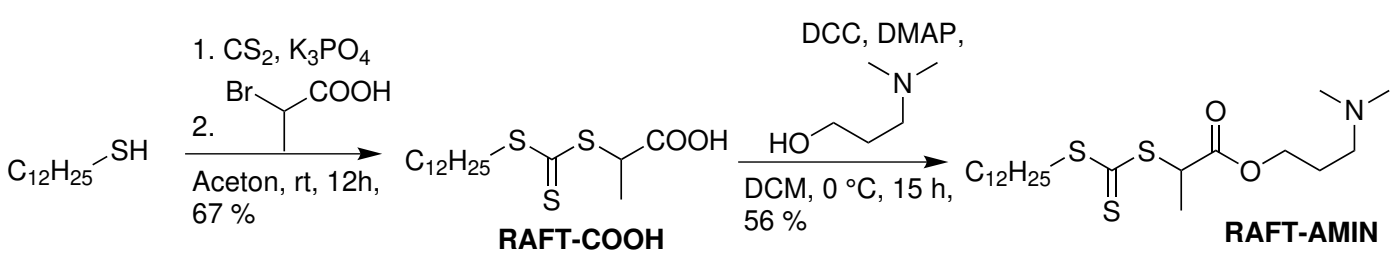

Schema 4.1. Synthese der nicht ionischen RAFT-Agenzien; DCM = Dichlormethan, $r t$ = Raumtemperatur. 


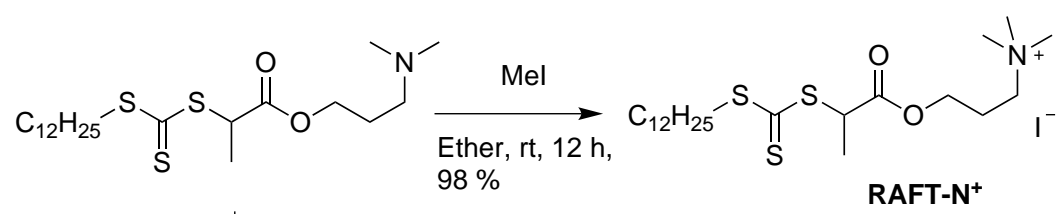<smiles>CCN(CC)Cc1ccc(CBr)cc1</smiles><smiles>CCSC(=S)SC(C)C(=O)OCCC[N+](C)(C)Cc1ccc(C[N+](CC)(CC)CC)cc1</smiles>

Schema 4.2. Synthese der ionischen RAFT-Agenzien.

\subsection{Synthese der RAFT-Polymere}

Zur besseren Übersichtlichkeit werden die Polymere entsprechend ihrer Endgruppe, Molmasse und Monomereinheit benannt. Ein Poly(Methylacrylat) (PMA), das mit dem RAFTAgens RAFT-COOH polymerisiert wurde und das eine Molmasse von $2000 \mathrm{~g} \mathrm{~mol}^{-1}$ hat, wird im Folgenden RAFT-PMA ${ }_{2 \mathrm{k}}-\mathrm{COOH}$ genannt. Eine analoge Nomenklatur wird für Polystyrol (PS) verwendet.

Mit den hergestellten RAFT-Agenzien wurden Polymere mit unterschiedlichen Molmassen hergestellt, um anschließend den Unterschied zwischen Polymeren mit und ohne ionische Endgruppen in der Massenspektrometrie untersuchen zu können. Dabei wurde Polystyrol als unpolares Polymer ohne Heteroatome und Poly(Methylacrylat) als polareres Polymer gewählt. Die Molmasse der Polymere wurde über das Verhältnis von Monomer zu RAFTAgens ([M]:[RAFT]) eingestellt, indem bei Styrol bis zu einem Umsatz von 20-30 \% und bei Methylacrylat bis nahezu vollem Umsatz polymerisiert wurde. Als Initiator (I) wurde immer Azobis(isobutyronitril) (AIBN) verwendet. Das Verhältnis von RAFT-Agens zum Initiator ([RAFT]:[I]) betrug in alle Fällen 10:1. Polymerisiert wurde bei 60 oder $70{ }^{\circ} \mathrm{C}$. Alle Polymerisationen wurden zum besseren Vergleich als Lösungspolymerisation durchgeführt, da die ionischen RAFT-Agenzien nicht im Monomer löslich sind.

Im Falle der Polymerisation mit ionischen RAFT-Agenzien wurde Dimethylformamid (DMF), sonst Butanon als Lösungsmittel verwendet. Schema 4.3 gibt einen Überblick über die verwendeten Reagenzien der durchgeführten Polymerisationen. PMA wurde durch Abdampfen des Lösungsmittels und des Monomers isoliert. Die geladenen PMA ließen sich nicht in Methanol $(\mathrm{MeOH})$ fällen, da sie vermutlich aufgrund der Ladung zur Ausbildung mizellarer Strukturen neigen, wofür auch die Schaumbildung der Lösung in $\mathrm{MeOH}$ spricht. Die Polystyrole wurden ebenfalls durch Abdampfen isoliert. 

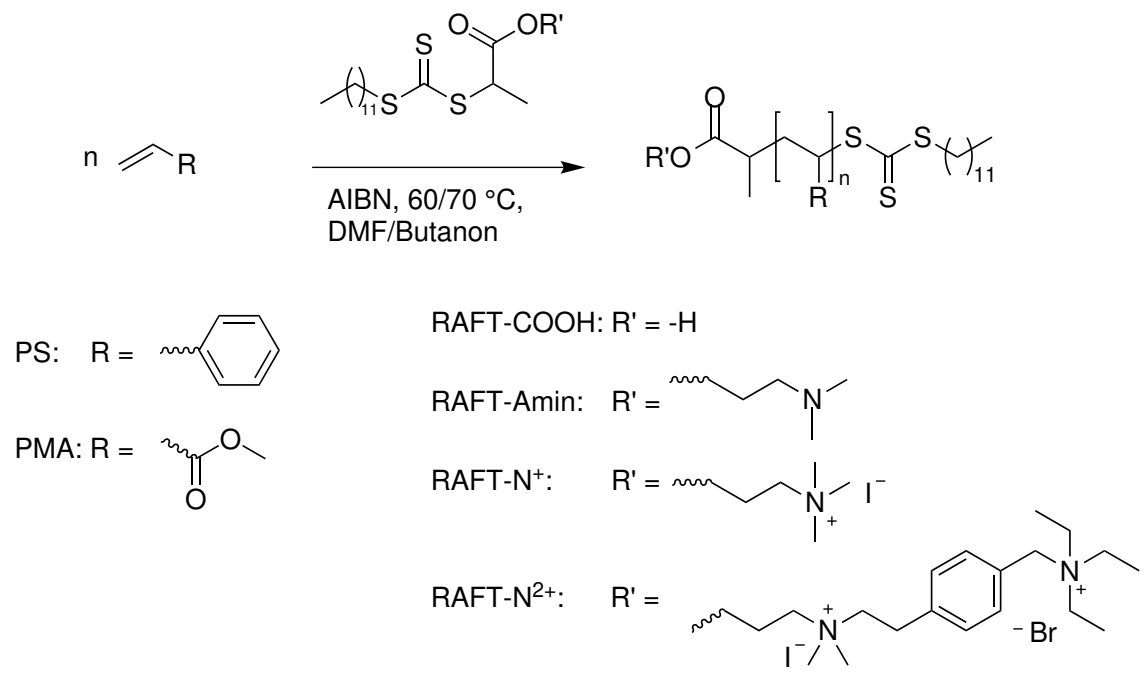

Schema 4.3. Synthese der ionischen und nicht-ionischen RAFT-Polymere.

Tab. 4.1. Daten der Polymerisationen mit nicht-ionischen RAFT-Agenzien. $T$ entspricht der Polymerisationstemperatur, $t$ der Polymerisationsdauer und $\mathrm{M}$ dem verwendeten Monomer. Butanon wurde als Lösungsmittel im Volumenverhältnis 1:1 zum Monomer verwendet.

\begin{tabular}{|c|c|c|c|c|c|c|c|}
\hline Probe & RAFT-Agens & [M]:[RAFT] & $t / \mathrm{h}$ & $T /{ }^{\circ} \mathrm{C}$ & $M_{\mathrm{n}}$ 的 & $E^{\natural}$ & $M_{\mathrm{n}, \text { theo }}$ \\
\hline RAFT-PMA ${ }_{2 k}-\mathrm{COOH}$ & RAFT-COOH & $25: 1$ & 16 & 60 & 2,0 & 1,1 & 2,0 \\
\hline RAFT-PMA $4 k-\mathrm{COOH}$ & RAFT-COOH & $50: 1$ & 5 & 60 & 3,8 & 1,1 & 4,2 \\
\hline RAFT-PMA ${ }_{7 k}-\mathrm{COOH}$ & $\mathrm{RAFT}-\mathrm{COOH}$ & 150:1 & 4,25 & 60 & 7,2 & 1,1 & - \\
\hline RAFT-PMA ${ }_{1 \mathrm{k}}-$ Amin & RAFT-Amin & $25: 1$ & 4,33 & 60 & 1,1 & 1,2 & 1,4 \\
\hline RAFT-PMA ${ }_{3 k}-A \min$ & RAFT-Amin & $50: 1$ & 8 & 60 & 3,4 & 1,1 & 3,4 \\
\hline RAFT-PMA $7 \mathrm{k}-\mathrm{Amin}$ & RAFT-Amin & 100:1 & 7,75 & 60 & 6,9 & 1,1 & - \\
\hline RAFT-PS $_{2 k}-\mathrm{COOH}$ & RAFT-COOH & $50: 1$ & 15 & 60 & 1,6 & 1,1 & 1,7 \\
\hline $\mathrm{RAFT}^{-P}{ }_{3 \mathrm{k}}-\mathrm{COOH}$ & $\mathrm{RAFT}-\mathrm{COOH}$ & $75: 1$ & 20 & 70 & 3,2 & 1,1 & 3,3 \\
\hline RAFT-PS ${ }_{2 k}-A \min$ & RAFT-Amin & $50: 1$ & 17 & 60 & 1,8 & 1,1 & 1,8 \\
\hline
\end{tabular}

${ }^{a}$ Bestimmt mittels GPC und der Verwendung der Mark-Houwink-Parameter von PMA/PS.

${ }^{b}$ In kg mol ${ }^{-1}$.

${ }^{c}$ Nach Gleichung 4.1 berechneter $M_{\mathrm{n}}$.

In Tabelle 4.1 sind die Daten der Polymerisationen mit den nicht-ionischen RAFT-Agenzien RAFT-COOH und RAFT-Amin zusammengefasst. RAFT-COOH und seine Ester, wie es RAFT-Amin ist, sind für die gute Kontrolle von Acrylaten und Styrol bekannt. 169/170] Dementsprechend liegt die Dispersität $\boxminus$ bei allen Polymeren zwischen 1,1 und 1,2 und die Molmassen stimmen mit den theoretischen Molmassen nach Gleichung 4.1 überein. In dieser Gleichung ist $U$ der Umsatz, $M_{\mathrm{M}}$ die Masse des Monomers, $M_{\text {RAFT }}$ die Masse des RAFT-Agens, $[M] /[R A F T]$ das anfängliche Verhältnis von Monomer zu RAFT-Agens.

$$
M_{\mathrm{n}}=M_{\mathrm{M}} \cdot U \cdot \frac{[M]}{[R A F T]}+M_{\mathrm{RAFT}}
$$


Tab. 4.2. Daten der Polymerisationen mit ionischen RAFT-Agenzien. DMF wurde als Lösungsmittel im Volumenverhätlnis 1:1 (8:5 bei RAFT-PMA $\left.{ }_{2 k}-\mathrm{N}^{2+}\right)$ zum Monomer verwendet.

\begin{tabular}{|c|c|c|c|c|c|c|c|}
\hline Probe & RAFT-Agens & $\mathrm{M}$ & [M]:[RAFT] & $t / \mathrm{h}$ & $T /{ }^{\circ} \mathrm{C}$ & $M_{\mathrm{n}} \mathrm{A}$ & $\Xi^{b}$ \\
\hline RAFT-PMA-N $^{+}$ & RAFT-N $^{+}$ & $M A$ & $15: 1$ & 10 & 60 & e & e \\
\hline RAFT-PMA $1 \mathrm{k}^{-\mathrm{N}^{+}}$ & - & MA & $25: 1$ & 4,33 & 60 & $1,1]^{c}$ & $1,2^{C}$ \\
\hline RAFT-PMA $3 \mathrm{~K}^{-}-\mathrm{N}^{+}$ & - & MA & $50: 1$ & 8 & 60 & $3,4^{\sqrt{c}}$ & $1,1 \mathrm{C}$ \\
\hline RAFT-PMA $_{7 \mathrm{k}}-\mathrm{N}^{+}$ & - & MA & $100: 1$ & 7,75 & 60 & $6,9 \mathbb{C}$ & $1,1 \mathrm{C}$ \\
\hline RAFT-PS ${ }_{2 k}-\mathrm{N}^{+}$ & - & Styrol & $50: 1$ & 17 & 60 & $1,8^{C}$ & $1,1 \mathrm{C}$ \\
\hline RAFT-PS $4 \mathrm{k}^{-\mathrm{N}^{+}}$ & RAFT-N $^{+}$ & Styrol & $150: 1$ & 20 & 70 & e & 回 \\
\hline RAFT-PS ${ }_{7 k}-\mathrm{N}^{+}$ & RAFT-N $^{+}$ & Styrol & $300: 1$ & 23 & 70 & e & e \\
\hline RAFT-PMA $2 k-\mathrm{N}^{2+}$ & RAFT-N ${ }^{2+}$ & MA & $25: 1$ & 4 & 60 & $2,39 p$ & $\theta$ \\
\hline RAFT-PMA ${ }_{1,6 \mathrm{k}}-\mathrm{N}^{2+}$ & RAFT-N $^{2+}$ & MA & $15: 1$ & 3 & 60 & 1,5 वैe & E \\
\hline
\end{tabular}

${ }^{a}$ In kg mol ${ }^{-1}$

${ }^{b}$ Bestimmt mittels GPC und der Verwendung der Mark-Houwink Paramter von PMA/PS.

${ }^{c}$ Methylierte Proben: Die $M_{\mathrm{n}}$-Werte beziehen sich auf die unmethylierten Polymere (Tabelle 4.1.

${ }^{d}$ Bestimmt über den Umsatz nach Gleichung 4.1

${ }^{e}$ Keine Molmassenbestimmung mittels GPC moglich aufgrund von Wechselwirkungen mit der Säule .

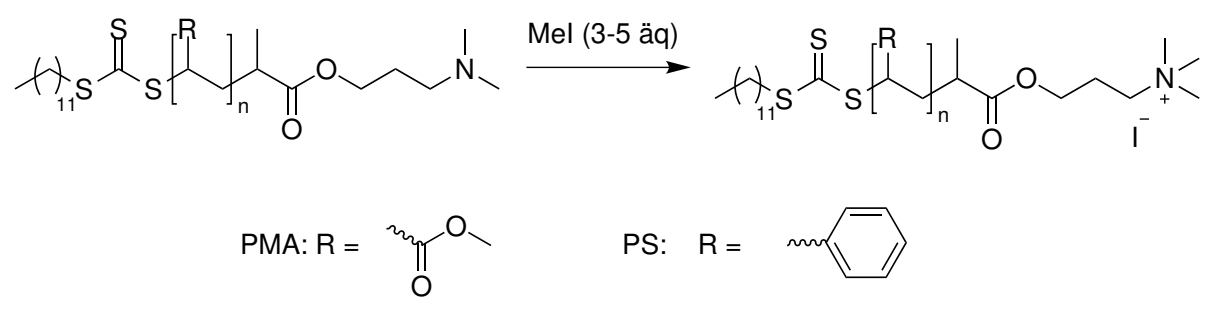

Schema 4.4. Methylierung der Dimethylaminoendgruppe nach der Polymerisation.

Tabelle 4.2 zeigt die Daten der Polymerisationen mit ionischen RAFT-Agenzien. Bei PMAPolymeren, die durch Polymerisation mit RAFT- $\mathrm{N}^{+}$hergestellt wurden (RAFT-PMA-N $\mathrm{N}^{+}$), war keine Bestimmung der MMV möglich. Im Elutionsdiagramm des Brechunsindexdetektors in Abbildung 4.1 A werden vor der Ausschlussgrenze kaum Analyten detektiert. Dafür entsteht ein sehr breiter Peak nach der Ausschlussgrenze der Säule ab ca. $38 \mathrm{ml}$. Es liegt die Vermutung nahe, dass das Polymer aufgrund der ionischen Gruppe mit dem Säulenmaterial wechselwirkt. Um diese Hypothese zu überprüfen wurden mehrere PMA (RAFT-PMA $1 k+3 k+7 k^{-}$ Amin) durch Polymerisation mit dem RAFT-Amin hergestellt und das tertiäre Amin in der Endgruppe entsprechend Schema 4.4 nach der Polymerisation methyliert. Anschließend wurden die, abgesehen von der Amin- und Ammoniumendgruppe, identischen Proben mit der GPC analysiert. Abbildung 4.1 B und $C$ zeigen die Elutionsdiagramme von RAFT-PMA $3 k^{-}$ Amin und der methylierten Probe RAFT-PMA $3 \mathrm{k}^{-} \mathrm{N}^{+}$. Das Polymer mit der nicht methylierten Endgruppe zeigt eine enge Molmassenverteilung, wie sie auch für die RAFT Polymerisationen mit RAFT-COOH üblich sind. Das Elutionschromatogramm der Probe RAFT-PMA $3 k^{-} \mathrm{N}^{+}$ hingegen ähnelt dem der Probe RAFT-PMA- $\mathrm{N}^{+}$, mit einem multimodalen Peak im Signal des UV-Detektors und einem Peak nach der Ausschlussgrenze. Es konnte also gezeigt werden, dass bereits eine einzige ionische Gruppe in der Polymerkette zu so starken Wech- 

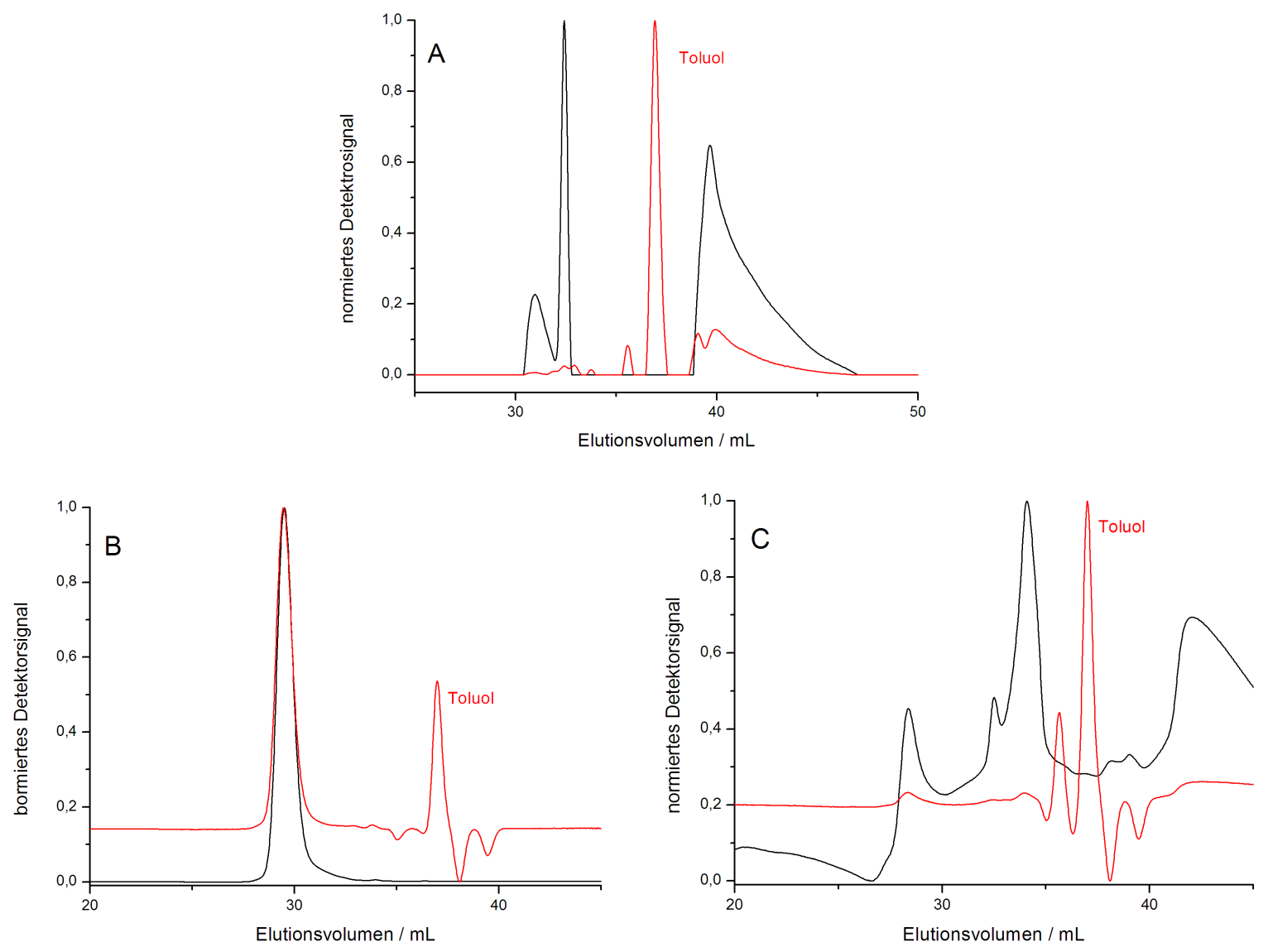

Abb. 4.1. GPC-Elutionschromatogramm von RAFT-PMA-N ${ }^{+}(A), R A F T-P M A_{3 k}-A m i n(B)$ und RAFT-PMA ${ }_{3 k}-\mathrm{N}^{+}(C)$. Die schwarzen Kurven zeigen das Signal des UV- und die roten Kurven das Signal des Brechungsindexdetektors.

selwirkungen mit der Säule der GPC führt, dass eine Auswertung der Molmassmassenverteilung mittels GPC nicht mehr sinnvoll ist. Abbildung 4.2 zeigt die NMR-Spektren von RAFT-PMA $11^{-}$-Amin und RAFT-PMA $1 \mathrm{k}^{-} \mathrm{N}^{+}$. Das Signal der zwei Methylgruppen am Stickstoff bei $\delta=2,12 \mathrm{ppm}$ von RAFT-PMA ${ }_{1 \mathrm{k}^{-}}$-Amin (blaues Spektrum) ist bei RAFT-PMA $1 \mathrm{k}^{-} \mathrm{N}^{+}$aufgrund der quantitativen Methylierung verschwunden. Stattdessen ist das Signal der Methylgruppen ins Tieffeld auf $\delta=3,08 \mathrm{ppm}$ verschoben worden. Die Reaktion ist bei den höher molekularen Polymeren RAFT-PMA $3 \mathrm{k} / 7 \mathrm{k}$-Amin ebenfalls quantitativ. Die Polystyrolproben mit ionischer Endgruppe (RAFT-PS $4 \mathrm{k} / 7 \mathrm{k}^{-} \mathrm{N}^{+}$) wechselwirken ebenfalls mit dem Säulenmaterial. Daher wurde für die Herstellung von RAFT-PS ${ }_{2 \mathrm{k}}-\mathrm{N}^{+}$das RAFT-Amin als RAFT-Agens eingesetzt, das Polymer mittels GPC charakterisiert und die Endgruppe anschließend analog zu den PMA-Proben nach der Polymerisation methyliert.

Die hier hergestellten Polymere RAFT-PMA $2 k+4 k+7 k-C O O H$ und RAFT-PS $2 k-C O O H$ ohne ionische Endgruppen weisen ähnliche Molmassen wie die Polymere mit ionischen Endgruppen RAFT-PMA $1 \mathrm{k}+3 \mathrm{k}+7 \mathrm{k}-\mathrm{N}^{+}$und RAFT-PS $2 \mathrm{k}^{-} \mathrm{N}^{+}$auf. Dadurch kann in den folgenden Abschnitte der Einfluss der ionischen Gruppen über einen großen Molmassenbereich und für das unpolare PS sowie das polarere PMA untersucht werden. 


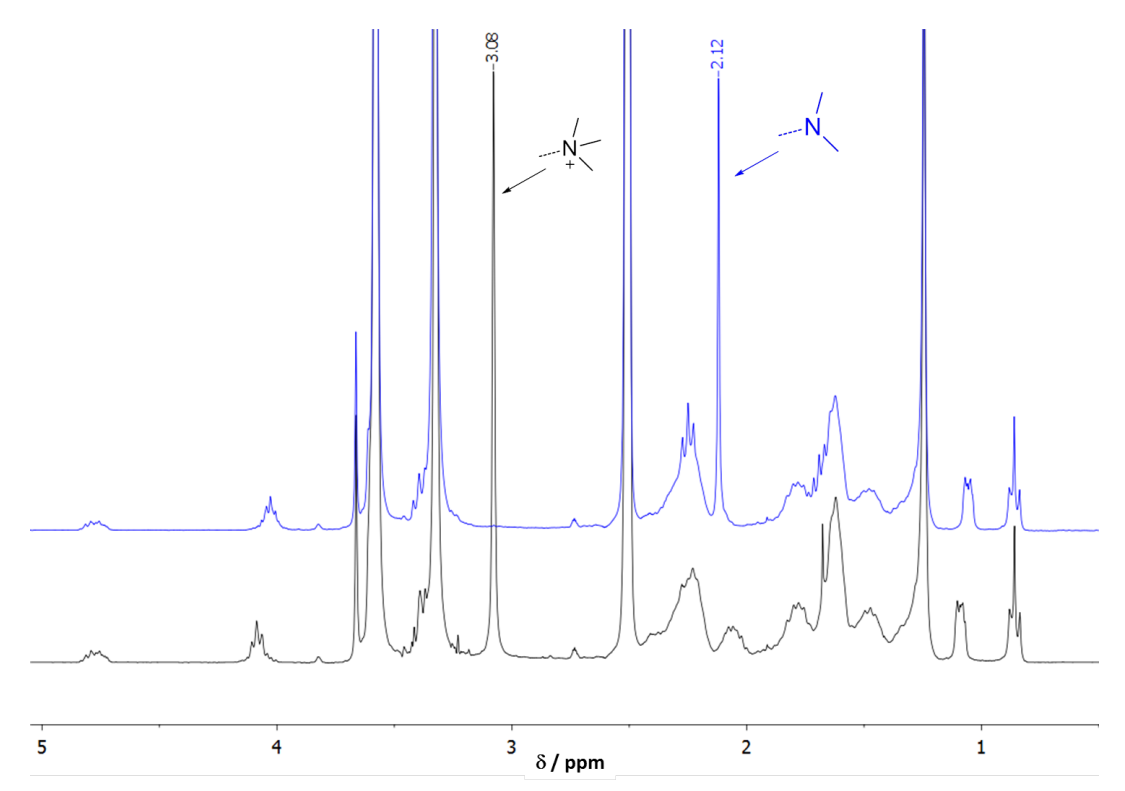

Abb. 4.2. NMR-Spektren von RAFT-PMA ${ }_{1 k}-A m i n$ (blau) und dem methylierten Polymer RAFT$\mathrm{PMA}_{1 \mathrm{k}}-\mathrm{N}^{+}$.

Entsprechend der Literatur und zu PE-N ${ }^{+}$aus Abschnitt 3.3 konnten die ionischen Endgruppen von RAFT-PS/PMA- $\mathrm{N}^{+}$auch durch die Phasentransfereigenschaften der RAFTPolymere nachgewiesen werden. ${ }^{153 / 154]}$ Dazu wurde eine wässrige Lösung von Methylorange (MO) mit Chloroform (Blindprobe) oder einer Lösung von RAFT-PMA $1 \mathrm{k}^{-\mathrm{N}^{+}}$in Chloroform versetzt. Die Chloroformphase ist in beiden Gefäßen zunächst farblos. Nach dem Schütteln verweilt das $\mathrm{MO}$ in der Blindprobe weiterhin in der wässrigen Phase, wohingegen der Farbstoff bei der RAFT-PMA $\mathrm{k}_{\mathrm{k}} \mathrm{N}^{+}$-Lösung vollständig in die organische Phase übergeht (Abbildung 4.3 A und B). Die Triebkraft für den Anionenaustausch von lodid (Gegenion im Polymer) und $\mathrm{MO}$ ist auch hier wieder die starke Solvatisierung des entstehenden $\mathrm{Nal}$ in der wässrigen Phase (vergleiche Schema 3.2 für PE- $\mathrm{N}^{+}$). Zudem ist anzunehmen, dass das MO

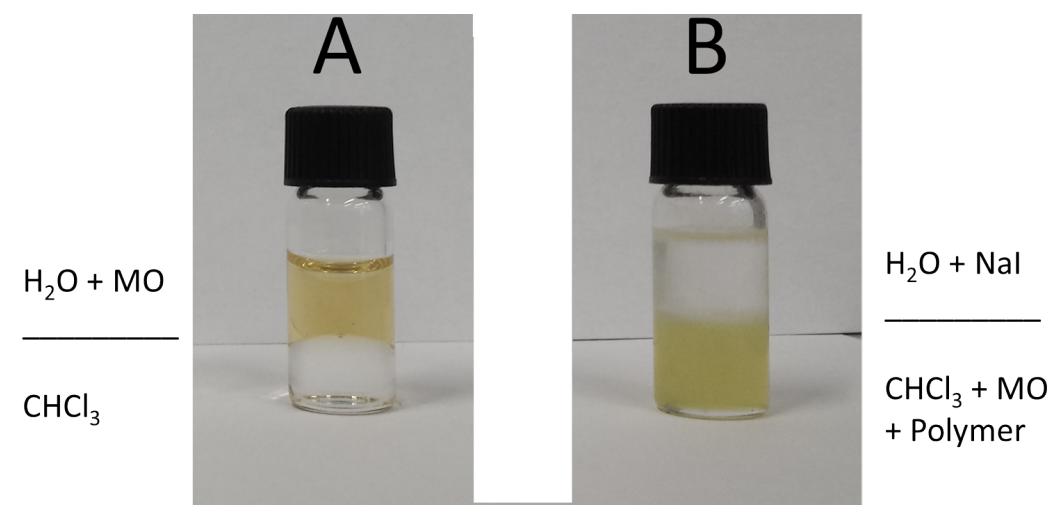

Abb. 4.3. Ein Zwei-Phasensystem nach Schütteln bestehend aus Chloroform und MO gelöst in Wasser (A) sowie ein Zwei-Phasensystem nach Schütteln bestehend aus RAFT-PMA ${ }_{1 k} \mathrm{~N}^{+}$und MO gelöst in Chloroform und Nalvgelöst in Wasser (B). 
aufgrund seiner organischen Struktur hydrophober als lodid ist und daher die organische Phase bevorzugt. Die Phasentransfereigenschaft der ionischen Endgruppe könnten, analog zu PE-N+ ${ }^{+}$, genutzt werden um neben Farbstoffen auch andere anionische Verbindungen wie nicht flüchtige Weichmacher oder Flammschutzmittel ins Polymer einzubauen. 159/160]

\subsection{ESI-MS Analyse der RAFT-Polymere}

Neben der Bestimmung des Einflusses diverser Geräteparameter in der ESI-MS lag der Fokus auf dem Vergleich von Polymeren mit und ohne ionischer Endgruppe. Dazu wurden die RAFT-Polymere aus den Tabellen 4.1 und 4.2 als 30 oder $50 \mu \mathrm{M}$ Lösung in Aceton ins Massenspektrometer injiziert. Die weiteren Messbedingungen können im Abschnitt 6.2 nachgelesen werden.

Um die Struktur der Endgruppen, den Polymerisationsgrad $n$ und das Adduktion der Peaks im Massenspektrum einfacher beschreiben zu können, wird die in Schema 4.5 A dargestellte Abkürzungsmethode verwendet. Schema $4.5 \mathrm{~B}$ zeigt die möglichen Abkürzungen für die Struktur der Endgruppen und Schema 4.5 C zeigt ein Beispiel für die Abkürzungsmethode samt der zugehörigen Polymerstruktur. Die Kürzel I und R entsprechen Polymerketten, deren Polymerisationen durch ein Initiatorfragment oder durch die R-Gruppe des RAFTAgens gestartet wurden. Im Gegensatz zur allgemeinen Nomenklatur der Z-Gruppe eines RAFT-Agens (Abbildung 2.1 A), beinhaltet $Z$ in dieser Notation die Z-Gruppe inklusive der Trithiocarbonatgruppe des RAFT-Agens. Endgruppen, in denen ein Schwefelatom oxidiert oder ein Schwefelatom durch ein Sauerstoffatom ausgetauscht wurde, werden OZ bzw. ZO benannt. Polymerketten, die durch Rekombination von zwei mittels R-Gruppen initiierten Ketten entstehen, erhalten das Kürzel RR. Besteht eine detektierte Spezies aus mehreren Polymerketten, so ist dies mit der ganzzahligen Zahl $\mathrm{X}$ vor dem Kürzel gekennzeichnet, sofern $X$ größer 1 ist. Wenn es um die allgemeine Beschreibung der Spezies geht, wird im Fließtext der Polymerisationsgrad nicht mit angegeben.

\subsubsection{Bestimmung der detektierten Spezies der Polymere}

Zunächst wurde untersucht, welche Spezies in den verschiedenen Polymeren mit und ohne ionische Endgruppe und in Abhängigkeit der Molmasse in der ESI-MS auftreten. Mit Spezies sind hierbei unterschiedliche Endgruppen und unterschiedliche Ladungszustände gemeint, wie in Schema 4.5 gezeigt.

In der Literatur gibt es mehrere detaillierte ESI-MS Untersuchungen zu den Endgruppen und Spezies von RAFT-Polymeren, die mit Hilfe von Dithiobenzoaten hergestellt wurden.164-166] Jedoch gibt es noch keine entsprechend detaillierten Untersuchungen für RAFT-Polymere, die Trithiocarbonaten enthalten, obwohl RAFT-Agenzenien mit Trithiocarbonatgruppen die Dithiobenzoate weitgehend verdrängt haben. Zwar gibt es vereinzelte ESI-Massenspektren dieser Polymere in der Literatur, aber diese weisen oft ein schlechtes Signal-zu-Rausch-Verhältnis auf und eine vollständige Analyse der Spektren fehlt. [171] In den nachfolgenden Massenspektren und deren Ausschnitten wird für eine verbesserte 
Übersichtlichkeit immer nur ein Signal einer Spezies beschriftet. Signale einer Spezies, die nicht zugeordnet werden konnten, werden im Massenspektrum mit „unbekannt“ gekennzeichnet.

A

Nomenklatur der Spezies in der MS

$\begin{aligned} \mathrm{q} & =\text { Anzahl Nettoladungen } \\ n & =\text { Polymerisationsgrad } \\ \mathrm{Ad} & =\text { Adduktspezies } \\ \mathrm{X} & =\text { detektiertes Signal besteht aus } \\ & \text { X Polymerketten; wird nur verwendet, } \\ & \text { wenn } \mathrm{X}>1\end{aligned}$

B

Kürzel Struktur des Polymers

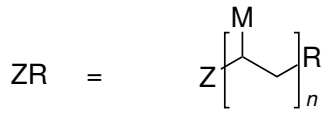

$\mathrm{zI}=\mathrm{z}$

$\mathrm{HR}=\mathrm{H}]_{n}^{\mathrm{M}}$

$\mathrm{OZR}=\mathrm{OZ}]_{n}^{\mathrm{M}}$

ZOR $=$ ZOY $]_{n}^{M}$

$\mathrm{RR}=\mathrm{R} \overbrace{n}^{\mathrm{M}}$

HOOR $=$ HOOH $]_{n}^{M}$

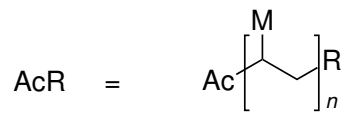

$\mathrm{UR}=\mathrm{R} \mathrm{H}_{\mathrm{n}-2}^{\mathrm{M} / \mathrm{M}}$

\section{C}

$\underline{\text { Beispiel }}$

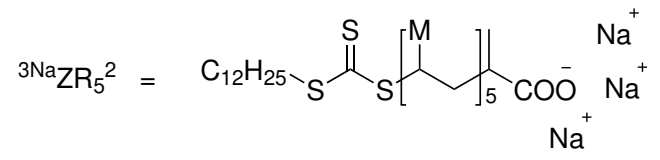

$$
\begin{aligned}
& \text { Abkürzungen }
\end{aligned}
$$

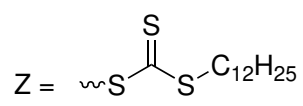

$$
\begin{aligned}
& \mathrm{OZ}=w \overbrace{S^{-}}^{S=O}
\end{aligned}
$$

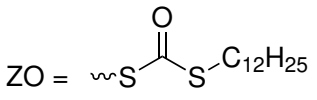

$$
\begin{aligned}
& M=m \text { oder mils } \\
& I=m-\mathrm{CN} \\
& A c=\text { m } \\
& \mathrm{H}=\sim \mathrm{H} \\
& \mathrm{HOO}=m \mathrm{O}^{-\mathrm{O}_{-}}
\end{aligned}
$$<smiles>[R]=C[C@H](C)C(=O)O</smiles>

oder<smiles>CC(C)C(=O)OCCC[N+](C)(C)C</smiles>

mit $\mathrm{A}=\mathrm{H}$ oder $\mathrm{CH}_{3}$

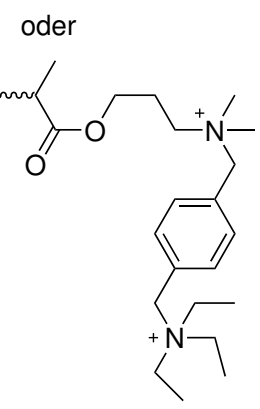

Schema 4.5. Nomenklatur für die verwendeten Abkürzungen der in der Massenspektrometrie detektierten Spezies (A), die Endgruppenstruktur der verschiedenen Kürzel (B) und ein Beispielkürzel samt Struktur $(C)$. 


\section{Speziesanalyse in Poly(Methylacrylat)}

Bei RAFT-PMA ${ }_{2 \mathrm{k}}-\mathrm{COOH}$ und RAFT-PMA $4 \mathrm{k}-\mathrm{COOH}$ werden die auftretenden Spezies detailliert anhand der Spektren untersucht. Abbildung $4.4 \mathrm{~A}$ zeigt das ESI-Massenspektrum einer acetonischen Lösung von RAFT-PMA ${ }_{2 k}-\mathrm{COOH}$ mit 4 äq Nal. Ohne Vergrößerung sind bereits die vier markierten Verteilungen ersichtlich. Es treten sowohl einfach geladene, als auch zweifach geladenen Spezies auf. In beiden Fällen und bei allen anderen hier untersuchten Polymeren bildeten die reversibel deaktivierten Polymerketten aus dem RAFTMechanismus die dominierende Spezies ${ }^{N a Z R}$. Die Abstände innerhalb der einfach geladenen Ketten betragen dabei $86 \mathrm{~m} / z$ und die der zweifach geladenen $43 \mathrm{~m} / \mathrm{z}$, was jeweils einer Monomereinheit entspricht. Zudem werden auch Ketten detektiert, in denen die Z-Gruppe inklusive der Trithiocarbonatgruppe durch ein $\mathrm{H}$-Atom ersetzt wurde (Spezies ${ }^{1,2 \mathrm{Na}} \mathrm{HR}^{1,2}$ ). Die HR-Spezies kann durch Disproportionierung der wachsenden Ketten entstehen. Da die Disproportionierung aber zur gleichen Anzahl von ungesättigten und $\mathrm{H}$-terminierten Kettenenden führt und ungesättigte Ketten auch in den Vergrößerungen des Massenspektrums 4.4 B und C) nicht gefunden werden, ist eine Entstehung der HR Spezies durch Fragmentation im ESI-Prozess wahrscheinlicher.
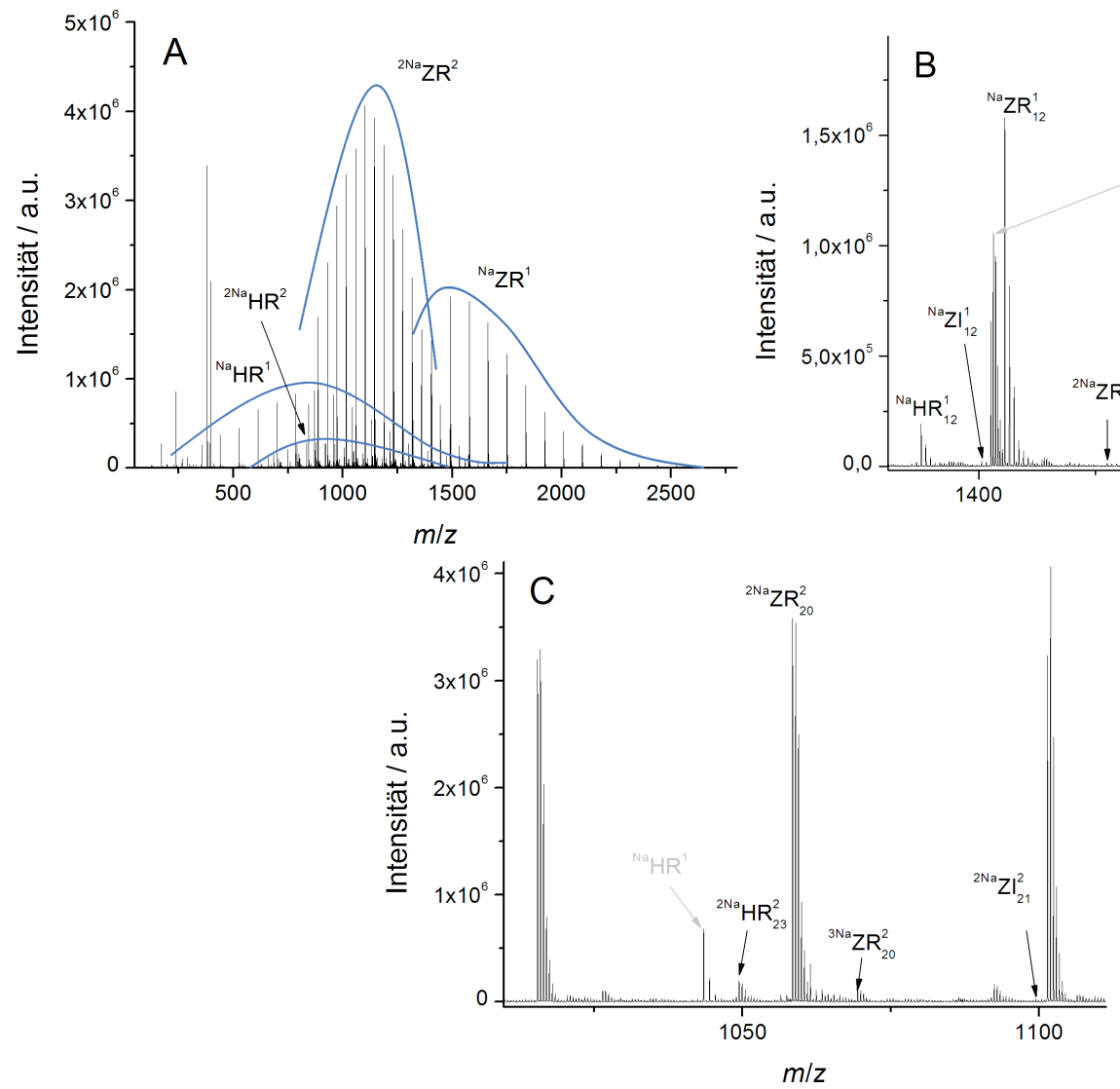

$m / z$

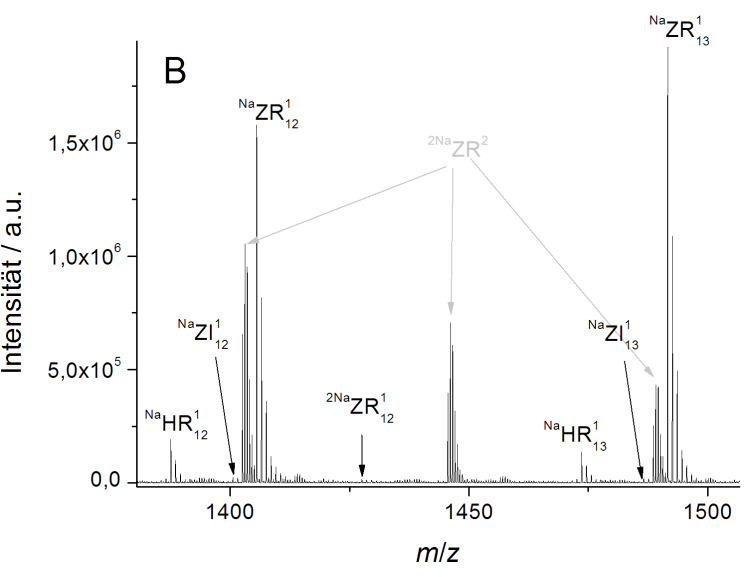

Abb. 4.4. Das ESI-Massenspektrum (A) von RAFT-PMA $A_{2 k}-\mathrm{COOH}$ gemessen in Aceton mit 4 äq Nal. Die Massenspektren B und C zeigen jeweils einen Ausschnitt des Massenspektrums A. 
Die Fragmentierung würde auch die Verschiebung der Ketten der HR zu kleineren $\mathrm{m} / \mathrm{z}$ gegenüber der ZR Spezies erklären. Aufgrund der kürzeren Ketten ist das Signal der einfach geladenen Spezies höher als das der zweifach geladenen, da weniger Bindungsstellen für ein zweites Natriumion zur Verfügung stehen. Die HR Spezies wurde bereits in der MALDIMS Analyse von Polyacrylsäure synthetisiert mit Trithiocarbonaten gefunden. 172[173]

Die Vergrößerung des Massenspektrums zeigt die Detektion weiterer PMA-Spezies. Abbildung 4.4 B zeigt einen Ausschnitt von 1380 bis $1500 \mathrm{~m} / \mathrm{z}$, der zur Analyse der einfach geladenen Spezies dient. Es tritt eine weitere einfach geladenen Spezies ${ }^{2 N a Z R^{1}}$ auf, bei der die endständige Carboxylgruppe als Natriumsalz vorliegt. Neben den einfach geladenen Spezies der Endgruppenstruktur HR und ZR werden auch Ketten detektiert, die durch ein Fragment des AIBN-Initiators initiiert wurden (Spezies ZI). Deren Intensität ist jedoch gering $(\mathrm{S} / \mathrm{R} \simeq 4)$.

Abbildung 4.4 C zeigt einen Ausschnitt mit den zweifach geladenen Spezies. Es werden die gleichen Endgruppen detektiert wie im Falle der einfach geladenen Spezies mit dem Unterschied, dass ein weiteres Natriumkation an die Polymerkette gebunden ist $\left({ }^{2 \mathrm{Na}} \mathrm{ZR}^{2}\right.$, ${ }^{2 N a} \mathrm{HR}^{2},{ }^{3 \mathrm{Na}} \mathrm{ZR}^{2}$ und ${ }^{2 \mathrm{Na}} \mathrm{ZI}^{2}$ ). Die Tabelle 4.3 beinhaltet eine Übersicht von allen gefundenen Spezies und zeigt die Summenformeln sowie die berechneten und experimentell ermittelten $\mathrm{m} / \mathrm{z}$-Werte für die in Abbildung 4.4 B und $\mathrm{C}$ markierten Monoisotopenpeaks. Die Abweichungen der experimentellen von den berechneten Werten ist geringer als $5 \mathrm{ppm}$, wodurch die korrekte Zuordnung der Signale unterstützt wird.

Tab. 4.3. Summenformeln, berechnete und experimentelle $m / z$-Werte der Monoisotopenpeaks der detektierten Spezies im ESI-Massenspektrum von RAFT-PMA ${ }_{2 k}-\mathrm{COOH}$ (Abb. 4.4). R entspricht $-\mathrm{CH}\left(\mathrm{CH}_{3}\right) \mathrm{COOH}$.

\begin{tabular}{|c|c|c|c|c|}
\hline Spezies & Summenformel & $\begin{array}{c}m / z \\
\text { berechnet }\end{array}$ & $\begin{array}{l}m / z \\
\text { gefunden }\end{array}$ & $\Delta^{a} / \mathrm{ppm}$ \\
\hline $\mathrm{Na}_{Z} \mathrm{R}_{13}$ & $\mathrm{C}_{68} \mathrm{H}_{108} \mathrm{O}_{28} \mathrm{~S}_{3} \mathrm{Na}^{+}$ & 1491,6081 & 1491,6024 & 4 \\
\hline $\mathrm{NaHR}^{1}{ }_{16}^{13}$ & $\mathrm{C}_{67} \mathrm{H}_{102} \mathrm{O}_{34} \mathrm{Na}^{+}$ & 1473,6145 & 1473,6198 & 4 \\
\hline${ }^{2 N a} Z{ }^{1}{ }_{12}$ & $\mathrm{C}_{64} \mathrm{H}_{101} \mathrm{O}_{26} \mathrm{~S}_{3} \mathrm{Na}_{2}^{+}$ & 1427,5533 & 1427,5566 & 2 \\
\hline $\mathrm{Na}_{\mathrm{ZI}}{ }_{13}{ }_{13}$ & $\mathrm{C}_{69} \mathrm{H}_{109} \mathrm{NO}_{26} \mathrm{~S}_{3} \mathrm{Na}^{+}$ & 1486,6292 & 1486,6337 & 3 \\
\hline $2 \mathrm{Na}_{Z} \mathrm{R}^{2}{ }_{20}$ & $\mathrm{C}_{96} \mathrm{H}_{150} \mathrm{O}_{42} \mathrm{~S}_{3} \mathrm{Na}_{2}{ }^{2+}$ & 1058,9291 & 1058,9285 & 0,6 \\
\hline${ }^{2 N a} \mathrm{HR}^{2}{ }_{23}$ & $\mathrm{C}_{95} \mathrm{H}_{144} \mathrm{O}_{48} \mathrm{Na}_{2}^{2+}$ & 1049,4306 & 1049,4336 & 3 \\
\hline $3 \mathrm{Na} \mathrm{ZR}^{2}{ }_{20}$ & $\mathrm{C}_{96} \mathrm{H}_{149} \mathrm{O}_{42} \mathrm{~S}_{3} \mathrm{Na}_{3}{ }^{2+}$ & 1069,4184 & 1069,4222 & 4 \\
\hline $2 \mathrm{Na}_{\mathrm{ZI}}^{2}{ }_{21}^{20}$ & $\mathrm{C}_{101} \mathrm{H}_{157} \mathrm{NO}_{42} \mathrm{~S}_{3} \mathrm{Na}_{2}{ }^{2+}$ & 1099,4580 & 1099,4623 & 4 \\
\hline
\end{tabular}

${ }^{a}$ Abweichungen der experimentell ermittelten von den berechneten Werten.

Um zu überprüfen, ob bei Polymeren mit höherer Molmasse durch Fragmentierung weitere Endgruppen auftreten, wurde auch das Polymer RAFT-PMA $4 \mathrm{k}-\mathrm{COOH}$ analysiert. Die Identifikation der detektierten Spezies im Massenspektrum von RAFT-PMA $4 \mathrm{k}^{-} \mathrm{COOH}$ in $\mathrm{Ab}$ bildung 4.5 ist schwieriger, da die Polymerketten aufgrund des höheren Polymerisationsgrades mehr Bindungsstellen für Natriumionen aufweisen. Daher werden mehr Ladungszustände (bis zu vierfach geladene Ketten) in der ESI-MS detektiert werden (Abb. 4.5 D). 
Um die Anzahl der Ladungen pro Kette zu reduzieren und die Interpretation des Spektrums zu erleichtern, wurde der Nal-Gehalt in der Polymerlösung auf zwei Äquivalente reduziert. Dadurch tritt auch die Spezies ${ }^{2 N a} Z_{R}{ }^{1}$ nicht mehr auf. Die absoluten Intensitäten der Peakmaxima sind bei gleicher Konzentration etwa halb so hoch wie bei RAFT-PMA ${ }_{2 k}-\mathrm{COOH}$ und das S/R-Verhältnis sinkt ebenfalls um $75 \%$. Ursache für die niedrigere Sensitivität des Massenspektrometers ist die geringere Transporteffizienz und das geringere Ansprechverhalten des Detektors für große Moleküle. 7017185

Es werden zu meist die bereits von RAFT-PMA ${ }_{2 k}-\mathrm{COOH}$ bekannten Spezies ZR, HR und ZI mit unterschiedlichen Ladungszuständen detektiert. Die ZI Spezies tritt nur im kleineren

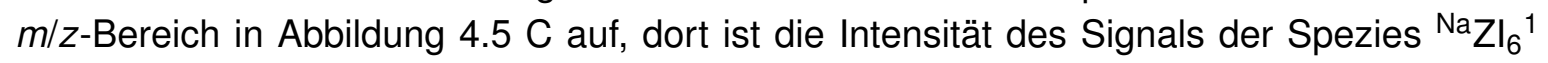
aber ähnlich intensiv wie das des ${ }^{\mathrm{Na}} \mathrm{ZR}_{6}{ }^{1}$. Das verstärkte Auftreten der ZI Spezies bei kürzeren Kettenlängen lässt sich dadurch erklären, dass durch den permanenten Zerfall des Initiators während der RAFT-Polymerisation ständig neue Ketten gestartet werden. Dadurch gibt es vor allem nach längeren Polymerisationzeiten viele kurzkettige Spezies ZI. Die durch die R-Gruppe des RAFT-Agens gestarteten Ketten wachsen hingegen schon seit Beginn der Polymerisation und sind dementsprechend am Ende der Polymerisation länger.
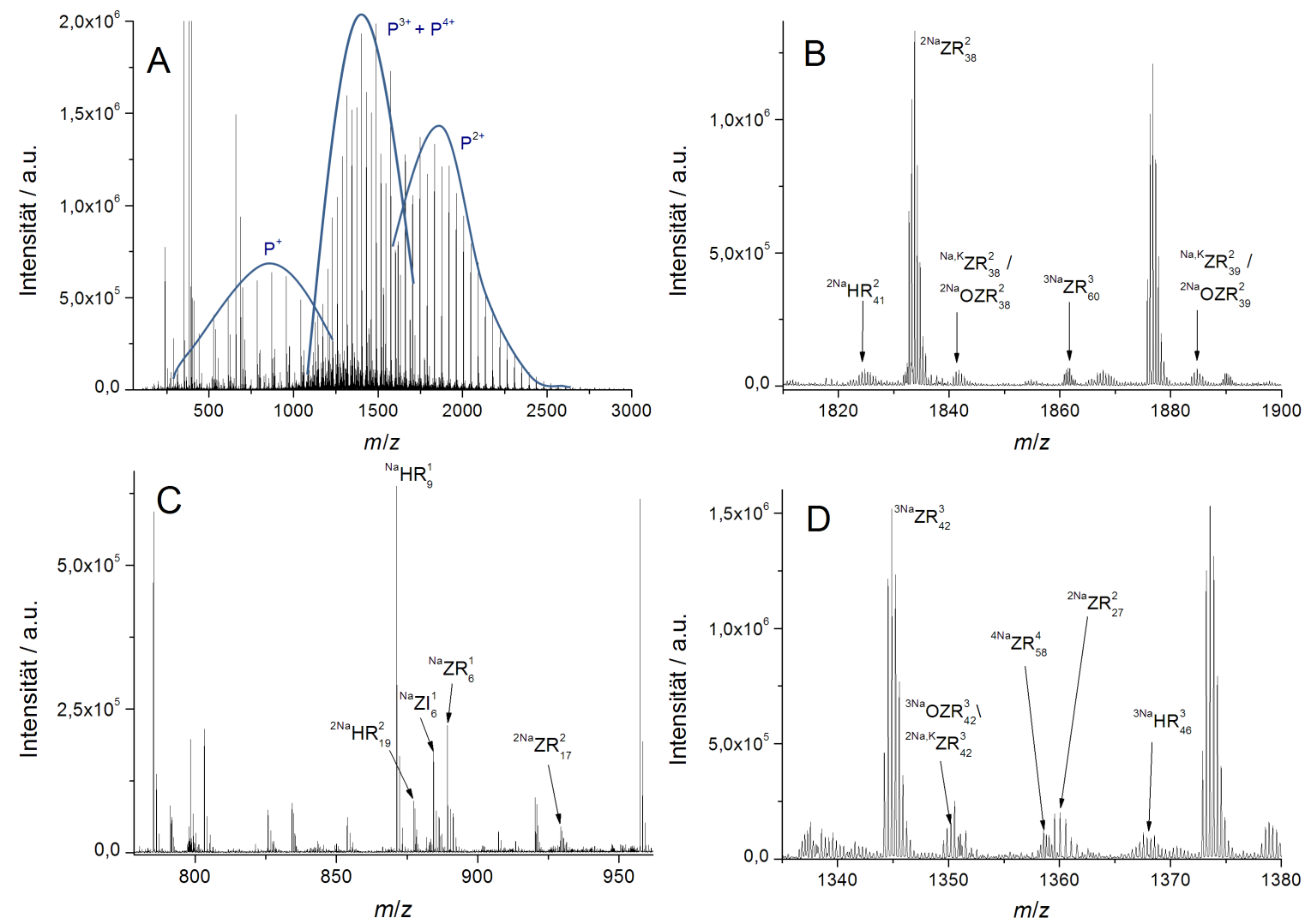

Abb. 4.5. Das ESI-Massenspektrum (A) von RAFT-PMA ${ }_{4 k}-\mathrm{COOH}$ gemessen in Aceton mit 2 äq $\mathrm{Nal}$. In den markierten Bereichen treten ein-, zwei- sowie drei- und vierfach geladene $\left(\mathrm{P}^{+}, \mathrm{P}^{2+}\right.$ etc.) Spezies auf. Die Massenspektren B bis D zeigen jeweils einen Ausschnitt des Massenspektrums. 
Zusätzlich wird eine zweifach geladene Spezies mit einer Verschiebung von $8 \mathrm{~m} / \mathrm{z}$ $\left(16 \mathrm{~g} \mathrm{~mol}^{-1}\right)$ gegenüber der ${ }^{2 \mathrm{Na} Z \mathrm{Z}^{2}}$ Spezies und eine dreifach geladene Spezies mit einer Verschiebung von 5,33 $\mathrm{m} / \mathrm{z}\left(16 \mathrm{~g} \mathrm{~mol}^{-1}\right)$ gegenüber der ${ }^{3 \mathrm{Na}} \mathrm{ZR}^{3}$ detektiert. Diese Signale können sowohl durch lonisierung von einem bzw. zwei Natriumionen und einem Kaliumion $\left.{ }^{(2) \mathrm{Na}, \mathrm{K}} \mathrm{ZR}^{2}\right)$, als auch durch die Oxidation des Schwefels von der C=S-Doppelbindung zu $\mathrm{C}=\mathrm{S}=\mathrm{O}\left({ }^{\left.2(3) \mathrm{Na} \mathrm{OZR}^{2}\right)}\right.$ entstehen. Die Oxidation des Schwefels in der ESI-MS wurde bereits für Dithioester beschrieben. ${ }^{164-166}$ Die Abweichungen der Signale sind, wie in Tabelle 4.4 gezeigt, geringer für die $\mathrm{m} / \mathrm{z}$-Werte der OZR Spezies. Auch für die nachfolgenden Polymere, in denen dieses Signal auftritt, sind die Abweichungen für die OZR Spezies geringer. Zudem werden am verwendeten Massenspektrometer keine Kaliumsalze verwendet. Die Tabelle 4.4 zeigt, dass die Abweichungen der experimentellen zu den theoretisch berechneten $\mathrm{m} / \mathrm{z}$-Werten mit maximal $7 \mathrm{ppm}$ ebenfalls sehr gering sind.

Tab. 4.4. Summenformeln, berechnete und experimentelle $m / z$-Werte des häufigsten Isotopenpeaks der detektierten Spezies im ESI-Massenspektrum von RAFT-PMA 4 - $-\mathrm{COOH}$ (Abb. 4.5). $\mathrm{R}$ entspricht $-\mathrm{CH}\left(\mathrm{CH}_{3}\right) \mathrm{COOH}$.

\begin{tabular}{|c|c|c|c|c|}
\hline Spezies & Summenformel & $\begin{array}{c}m / z \\
\text { berechnet }\end{array}$ & $\begin{array}{c}m / z \\
\text { gefunden }\end{array}$ & $\Delta^{\sqrt[a]{a} / p p m}$ \\
\hline $\mathrm{NaZR}_{6}{ }_{6}$ & $\mathrm{C}_{40} \mathrm{H}_{66} \mathrm{O}_{14} \mathrm{~S}_{3} \mathrm{Na}^{+}$ & 889,3507 & 889,3483 & -3 \\
\hline${ }^{2 N a} Z^{2}{ }^{2}{ }_{17}$ & $\mathrm{C}_{84} \mathrm{H}_{132} \mathrm{O}_{36} \mathrm{~S}_{3} \mathrm{Na}_{2}{ }^{2+}$ & 927,3722 & 927,3720 & $-0,2$ \\
\hline $\mathrm{Na}_{\mathrm{ZI}}{ }_{6}$ & $\mathrm{C}_{41} \mathrm{H}_{67} \mathrm{NO}_{12} \mathrm{~S}_{3} \mathrm{Na}^{+}$ & 884,3718 & 884,3699 & -2 \\
\hline $\mathrm{NaHR}_{9}^{1}$ & $\mathrm{C}_{39} \mathrm{H}_{60} \mathrm{O}_{20} \mathrm{Na}^{+}$ & 871,3570 & 871,3602 & 4 \\
\hline${ }^{2 \mathrm{Na}} \mathrm{HR}^{2}{ }_{19}$ & $\mathrm{C}_{79} \mathrm{H}_{120} \mathrm{O}_{40} \mathrm{Na}_{2}{ }^{2+}$ & 877,3570 & 877,3617 & 5 \\
\hline $\mathrm{Na}, \mathrm{K}_{\mathrm{ZR}^{2}{ }_{38}}$ & $\mathrm{C}_{169} \mathrm{H}_{260} \mathrm{O}_{78} \mathrm{~S}_{3} \mathrm{KNa}^{2+}$ & 1841,7487 & 1841,7610 & 7 \\
\hline${ }^{2 \mathrm{Na}} \mathrm{OZR}^{2}{ }_{38}$ & $\mathrm{C}_{169} \mathrm{H}_{260} \mathrm{O}_{79} \mathrm{~S}_{3} \mathrm{Na}_{2}{ }^{2+}$ & 1841,7592 & 1841,7610 & 1 \\
\hline${ }^{3} \mathrm{Na}_{Z^{2} \mathrm{R}_{60}^{3}}$ & $\mathrm{C}_{256} \mathrm{H}_{390} \mathrm{O}_{122} \mathrm{~S}_{3} \mathrm{Na}_{3}{ }^{3+}$ & 1861,4418 & 1861,4461 & -2 \\
\hline${ }^{3 \mathrm{Na}} \mathrm{HR}^{3}{ }_{46}$ & $\mathrm{C}_{187} \mathrm{H}_{282} \mathrm{O}_{94} \mathrm{Na}_{3}{ }^{3+}$ & 1367,5677 & 1367,5621 & 4 \\
\hline${ }^{4 N a} Z^{4}{ }_{58}$ & $\mathrm{C}_{248} \mathrm{H}_{378} \mathrm{O}_{118} \mathrm{~S}_{3} \mathrm{Na}_{4}{ }^{4+}$ & 1358,5594 & 1358,5541 & 4 \\
\hline
\end{tabular}

${ }^{a}$ Abweichungen der experimentell ermittelten von den berechneten Werten.

Die Massenspektren der weiteren auf ihre Spezies hin untersuchten PMA-Polymere und die zugehörigen Tabellen mit den Abweichungen der experimentell gefundenen $\mathrm{m} / \mathrm{z}$-Werte von den theoretisch berechneten Werten finden sich im Anhang und sind für die Polymere in Tabelle 4.5 aufgelistet. Tabelle 4.5 gibt zudem eine Übersicht, welche Spezies für das jeweilige Polymer gefunden wurden. Im Folgenden wird nur noch auf Unterschiede der Polymere gegenüber RAFT-PMA $2 \mathrm{k}-\mathrm{COOH}$ und RAFT-PMA $4 \mathrm{k}-\mathrm{COOH}$ eingegangen.

Bei RAFT-PMA ${ }_{1 k}$-Amin wurde statt eines Natriumsalzes Trifluoressigsäure zugegeben, um die endständige Aminogruppe zu protonieren. Das Massenspektrum in Abbildung A3 im Anhang zeigt, dass gegenüber RAFT-PMA ${ }_{2 \mathrm{k}}-\mathrm{COOH}$ der Anteil an zweifach geladenen Ketten aufgrund des niedrigeren Polymerisationsgrades wesentlich geringer ist. Die zweifach geladenen Ketten werden durch ein Proton und ein Natriumion ionisiert. Die einfach geladenen Spezies sind bevorzugt über die Protonierung ionisiert, teils aber auch durch Adduktbildung mit dem omnipräsenten Natriumionen. Dadurch, dass die Ionisation durch $\mathrm{H}^{+}$ 
oder $\mathrm{Na}^{+}$möglich ist und durch die Entstehung von vier unbekannten Spezies, wird das Spektrum komplexer. Die Möglichkeit der Protonierung führt auch nicht zu einem besseren S/R-Verhältnis, weshalb eine terminale Aminogruppe für die Analyse von PMA in der ESI-MS nachteilig ist.

Die Polymere RAFT-PMA $A_{1 k^{-}}-\mathrm{N}^{+}$und RAFT-PMA $\mathrm{A}_{3 \mathrm{k}}-\mathrm{N}^{+}$benötigen zur lonisierung weder ein Salz noch eine Säure, da die endständige Ammoniumgruppe bereits eine Ladung trägt. Mehrfach geladene Ketten entstehen jedoch auch durch Adduktbildung mit Natriumionen wie in Tabelle 4.5 und in den Massenspektren im Anhang A4 und A5 gezeigt. Bei RAFT$\mathrm{PMA}_{3 \mathrm{k}}-\mathrm{N}^{+}$tritt eine neue Spezies ${ }^{\mathrm{Na} Z R O^{2}}$ auf, bei der ein Schwefelatom der Trithiocarbonatgruppe durch ein Sauerstoffatom ersetzt wurde. Dieser Schwefel-Sauerstoff-Austausch entsteht unter anderem durch die Oxidierung der Trithiocarbonatgruppe mit Peroxiden und wurde bisher nur bei der ESI-MS von Polymeren mit Dithiobenzoaten beobachtet. 164-166] Die Tabelle B2 im Anhang zeigt anhand der einfach geladenen ZR Spezies, dass mit steigender Kettenlänge die Abweichungen zwischen den berechneten und experimentellen $\mathrm{m} / \mathrm{z}$-Werten zunimmt. Dies ist darauf zurückzuführen, dass das Massenspektrometer nur bis $2000 \mathrm{~m} / \mathrm{z}$ kalibriert wurde.

Das Polymer RAFT-PMA ${ }_{2 k}-\mathrm{N}^{2+}$ besitzt eine zweifach geladene Endgruppe und dementsprechend werden nahezu ausschließlich zweifach geladenen Ketten der Spezies $Z^{2}$ und $\mathrm{HR}^{2}$ detektiert. Im höheren $\mathrm{m} / \mathrm{z}$-Bereich werden auch Spuren zweier einfach geladener Spezies detektiert (Abb. A6 B im Anhang). Um eine einfach geladene Spezies zu erhalten, muss die Ladung eines Ammoniums durch ein Gegenion neutralisiert werden. Mit Hilfe der in der Endgruppe enthaltenen Gegenionen lodid und Bromid (siehe Schema 4.2 und 4.3) sowie weiterer Anionen (Hydroxid, Nitrat, Acetat, Chlorid etc.) konnte jedoch keine Struktur gefunden werden, die mit den $\mathrm{m} / \mathrm{z}$-Werten der beiden einfach geladenen Spezies in Abbildung A6 B übereinstimmt.

Tab. 4.5. Gefundene Spezies der weiteren untersuchten PMA und Anmerkungen zu den Messbedingungen. Die Spalte „Zusatzmaterial“ verweist auf die Massenspektren der Polymere und auf die Tabellen mit den $\mathrm{m} / \mathrm{z}$-Werten, die sich im Anhang befinden.

\begin{tabular}{|c|c|c|c|}
\hline Polymer & Messbedingungen & Spezies & $\begin{array}{l}\text { Zusatz- } \\
\text { material }\end{array}$ \\
\hline RAFT-PMA ${ }_{1 k}-A \min$ & Säurezugabe & $\begin{array}{c}\mathrm{H}_{\mathrm{ZR}}{ }^{1},{ }^{\mathrm{H}} \mathrm{HR}^{1},{ }^{\mathrm{Na}} \mathrm{HR}^{1},{ }^{\mathrm{Na}} \mathrm{ZR}^{1}, \\
\mathrm{H}, \mathrm{Na}^{2} \mathrm{R}^{2} ; 4 \text { unbekannte } \\
\text { einfach geladene }\end{array}$ & $\begin{array}{c}\text { Abb. } \mathrm{A3}, \\
\text { Tab. B1 }\end{array}$ \\
\hline RAFT-PMA ${ }_{1 \mathrm{k}}-\mathrm{N}^{+}$ & $\begin{array}{l}\text { keine Zugabe von } \\
\mathrm{Nal} / \text { Säure }\end{array}$ & 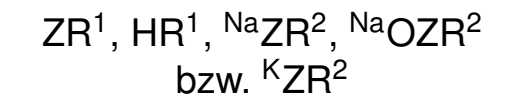 & $\begin{array}{l}\text { Abb. } \overline{\mathrm{A} 5}, \\
\text { Tab. B3 }\end{array}$ \\
\hline RAFT-PMA $3 \mathrm{k}^{-} \mathrm{N}^{+}$ & $\begin{array}{l}\text { keine Zugabe von } \\
\text { Nal/Säure }\end{array}$ & 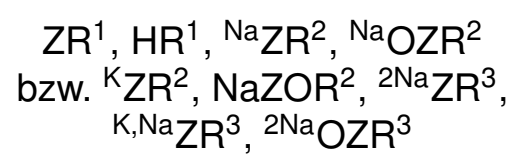 & $\begin{array}{r}\text { Abb. } \overline{\mathrm{A} 4}, \\
\text { Tab. } \mathrm{B2}\end{array}$ \\
\hline RAFT-PMA $2 k^{-} \mathrm{N}^{2+}$ & $\begin{array}{c}\text { keine Zugabe von } \\
\text { Nal/Säure }\end{array}$ & $\mathrm{ZR}^{2}, \mathrm{HR}^{2}$ & $\begin{array}{l}\text { Abb. } \overline{\mathrm{A} 6}, \\
\text { Tab. } \overline{\mathrm{B} 4}\end{array}$ \\
\hline
\end{tabular}




\section{Speziesanalyse in Polystyrol}

Die Tabelle 4.6 gibt einen Überblick über die gefundenen Spezies der vier mittels ESI-MS untersuchten Polystyrole. In diesem Abschnitt wird nicht mehr auf bereits von den PMA bekannte Spezies eingegangen, es sei denn es gibt signifikante Unterschiede. Die Polymere RAFT-PS ${ }_{3 \mathrm{k}}-\mathrm{COOH}$ und RAFT-PS ${ }_{2 \mathrm{k}}$-Amin wurden mit einer Kapillarspannung von $4,5 \mathrm{kV}$ statt $3,0 \mathrm{kV}$ gemessen. Die Messung ließ sich zu einem späteren Zeitpunkt nicht mehr mit einer Spannung von 3,0 kV wiederholen, da die Sensitivität des Spektrometers mit der Zeit stark nachließ. Ursache dafür ist vermutlich ein Problem mit dem Detektor. In Abschnitt 4.4.3 wird gezeigt, dass die erhöhte Kapillarspannung zu mehr Fragmentierung und zu einem schlechteren S/R-Verhältnis führen kann. Daher sind die Spektren dieser beiden Polymere nicht direkt mit den anderen Polymerspektren zu vergleichen.

Tab. 4.6. Gefundene Spezies der weiteren untersuchten PS und Anmerkungen zu den Messbedingungen. Die Spalte „Zusatzmaterial“ verweist auf die Massenspektren der Polymere und auf die Tabellen mit den $\mathrm{m} / \mathrm{z}$-Werten, die sich im Anhang befinden.

\begin{tabular}{|c|c|c|c|}
\hline Polymer & Messbedingungen & Spezies & $\begin{array}{l}\text { Zusatz- } \\
\text { material }\end{array}$ \\
\hline RAFT-PS ${ }_{2 k}-\mathrm{COOH}$ & Nal Zugabe & 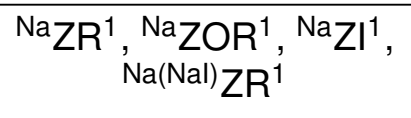 & $\begin{array}{l}\text { Abb. } 4.6 \\
\text { Tab. B5 }\end{array}$ \\
\hline 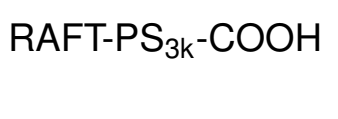 & $\begin{array}{c}\text { ", 4,5 kV } \\
\text { Kapillarspannung }\end{array}$ & $\begin{array}{l}\mathrm{Na}_{\mathrm{ZR}}{ }^{1},{ }^{\mathrm{Na}} \mathrm{HR}^{1}{ }^{1} \mathrm{Na}_{\mathrm{Z}}{ }^{1}, \\
\mathrm{Na}(\mathrm{Nal}) \mathrm{ZR}^{1}, \mathrm{Na}(\mathrm{Nal})_{2} \mathrm{ZR}^{1}\end{array}$ & $\begin{array}{c}\text { Abb. A77 } \\
\text { Tab. B6 }\end{array}$ \\
\hline RAFT-PS ${ }_{2 k}-A \min$ & $\begin{array}{c}\text { Säurezugabe, 4,5 kV } \\
\text { Kapillarspannung }\end{array}$ & $\begin{array}{l}{ }^{H} Z^{1}, 5 \text { unbekannte } \\
\text { einfach geladene }\end{array}$ & $\begin{array}{l}\text { Abb. } 4.7 \\
\text { Tab. B7 }\end{array}$ \\
\hline RAFT-PS $2 k-N^{+}$ & $\begin{array}{c}\text { keine Zugabe von } \\
\text { Nal/Säure }\end{array}$ & $\begin{array}{c}\mathrm{ZR}^{1}, \mathrm{HR}^{1}, \mathrm{RR}^{2}, 2 \mathrm{ZR} \mathrm{R}^{2}, \\
3 \mathrm{ZR}^{3}\end{array}$ & $\begin{array}{r}\text { Abb. } \overline{4.8} \\
\text { Tab. } \overline{\text { BB7 }}\end{array}$ \\
\hline
\end{tabular}

Bei den Polystyrolen RAFT-PS ${ }_{2 k}-\mathrm{COOH}$ und RAFT-PS ${ }_{3 k}-\mathrm{COOH}$ ohne Amin- bzw. Ammoniumgruppe wurde zur lonisation ebenfalls Nal verwendet. Das Natriumion wechselwirkt zwar wesentlich schwächer mit den $\pi$-Elektronen der Phenylgruppen als Übergangsmetalle wie Silber und Kupfer, aber diese Übergangsmetalle führen aufgrund ihrer hohen Affinität zu Schwefel auch zur Abspaltung der Trithiocarbonatgruppe. 174-176

Die Massenspektren von RAFT-PS ${ }_{2 k}-\mathrm{COOH}$ und RAFT-PS ${ }_{3 \mathrm{k}}-\mathrm{COOH}$ in Abbildung $4.6 \mathrm{bzw}$. A7 (Anhang) und Tabelle 4.6 zeigen, dass keine zweifach geladenen Polymerketten auftreten. Das kann an der geringeren Affinität von Polystyrol zur Komplexierung von Natrium liegen. Eventuell kann die Abstoßung zwischen zwei Natriumkationen nicht durch die schwache Interaktion mit $\pi$-Elektronen kompensiert werden. Stattdessen entstehen aus dem Überschuss an Nal Cluster der allgemeinen Form $\mathrm{Na}(\mathrm{Nal})^{+} \mathrm{x}$. Zudem werden auch Polystyrolketten des reversibel desaktivierten Spezies ZR durch Adduktbildung mit $\mathrm{Na}(\mathrm{Nal})$ oder $\mathrm{Na}(\mathrm{Nal})_{2}$ ionisiert. Diese lonisierung der Polystyrolketten durch $\mathrm{Na}(\mathrm{Nal})$-Cluster wurde auch in der Literatur gefunden. ${ }^{177]}$ Bei RAFT-PS ${ }_{3 k}-\mathrm{COOH}$ wird noch eine unbekannte 

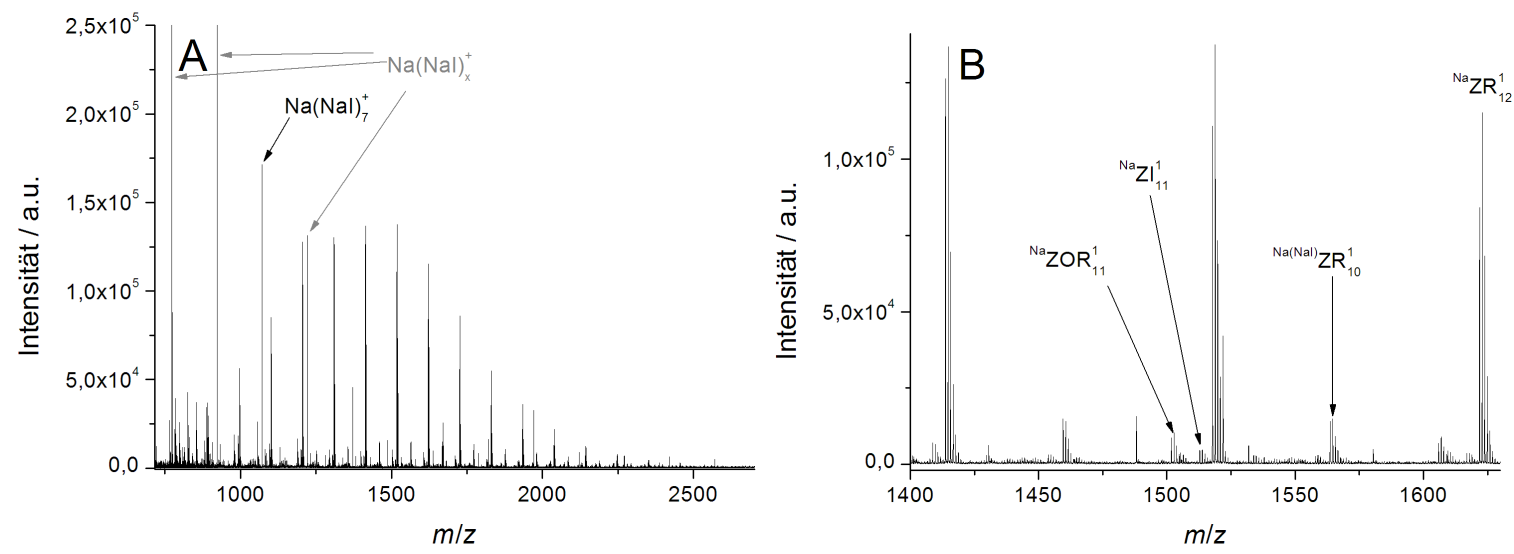

Abb. 4.6. Das vollständige ESI-Massenspektrum (A) und ein Ausschnitt (B) des Spektrums von RAFT-PS ${ }_{2 k}-\mathrm{COOH}$ gemessen in Aceton mit 10 äq Nal.

Spezies detektiert, was aber auf die höhere Kapillarspannung zurückzuführen sein könnte.

Die Lösung von RAFT-PS ${ }_{2 k}$-Amin wurde analog zu der Lösung von RAFT-PMA $11_{1}-$ Amin mit Trifluoressigsäure versetzt, um das Polymer durch Protonierung zu ionisieren. Bei dieser PS-Probe konnte nur die ${ }^{\mathrm{H}}$ ZR Spezies identifiziert werden (Abb. 4.7). Im Gegensatz zu RAFT-PMA ${ }_{1 k}$-Amin treten keine Polymerketten auf, die mit Natrium ionisiert wurden. Zudem werden noch fünf weitere Spezies detektiert, deren Struktur nicht identifiziert werden konnte. Diese könnten durch die höhere Kapillarspannung entstanden sein, jedoch traten unbekannte Spezies auch bei RAFT-PMA ${ }_{1 k}$-Amin auf. Die Einführung einer tertiären Aminendgruppe liefert bei Polystyrol analog zu PMA keinen Vorteil, da unbekannte Spezies entstehen und das Spektrum entsprechend komplexer wird. Das S/R-Verhältnis steigt bei RAFT-PS $2 \mathrm{k}-$ Amin gegenüber RAFT-PS ${ }_{2 k}-\mathrm{COOH}$ nicht an, sodass die Protonierung der Endgruppe bei der Sensitivität ebenfalls keinen entscheidenden Vorteil bringt.
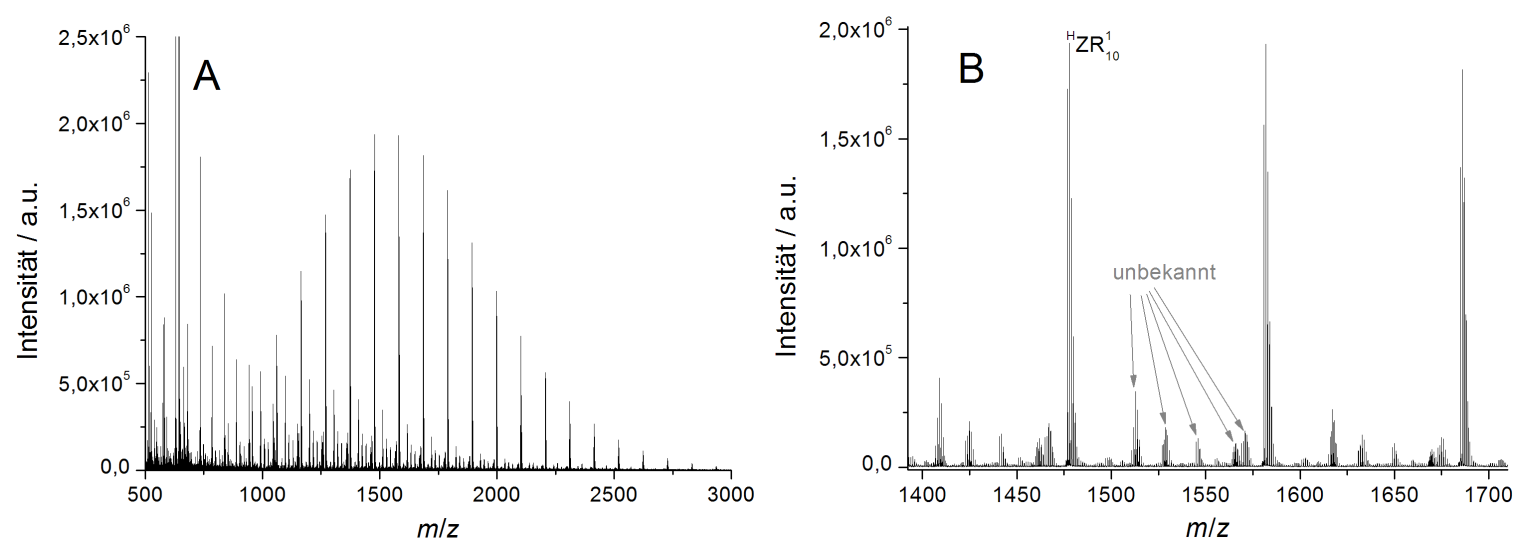

Abb. 4.7. Das komplette ESI-Massenspektrum (A) und ein Ausschnitt (B) des Spektrums von RAFT-PS ${ }_{2 \mathrm{k}}$-Amin gemessen in Aceton mit 0,1 wt\% Trifluoressigsäure. 

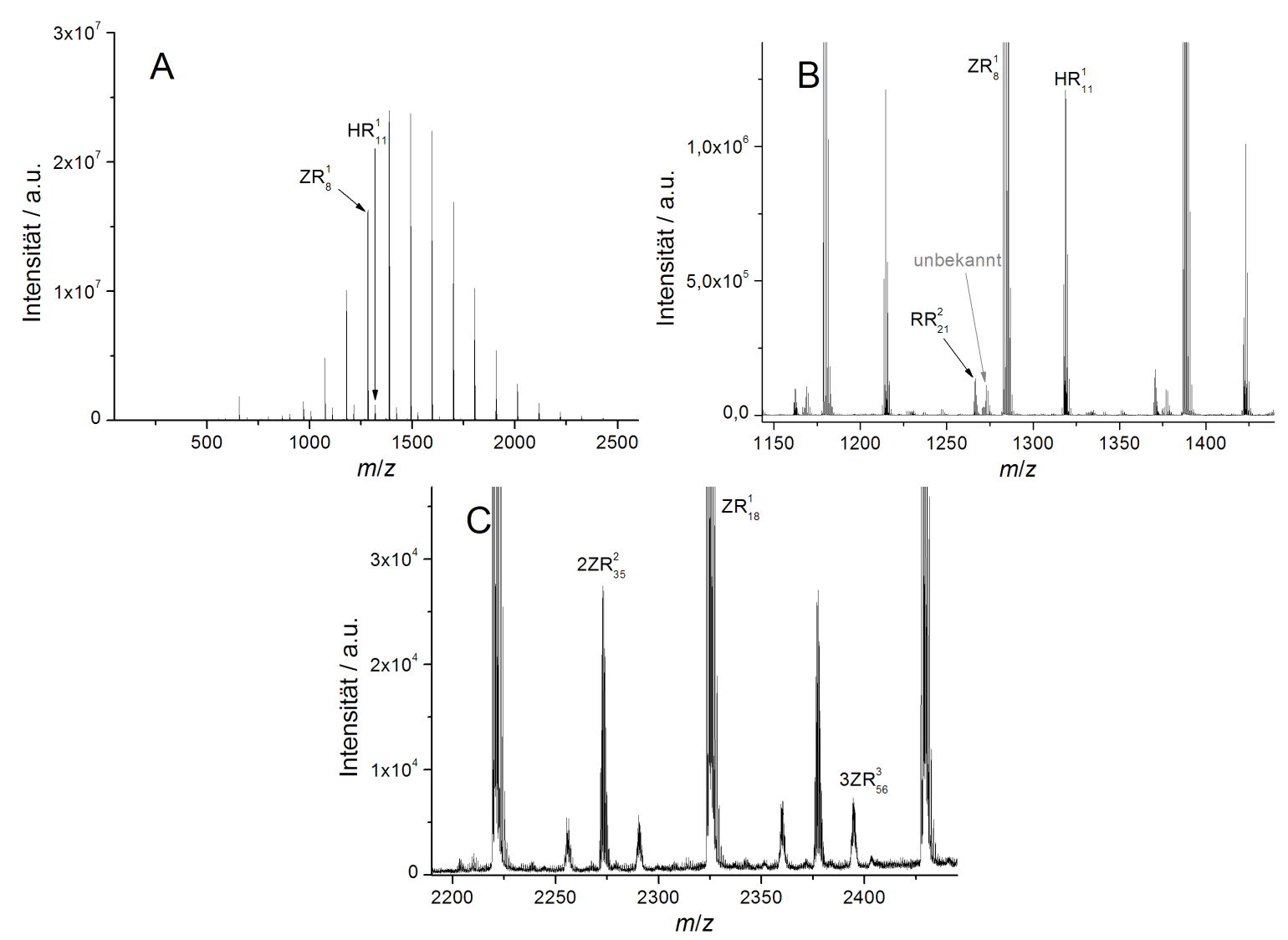

Abb. 4.8. Das komplette ESI-Massenspektrum (A) und Ausschnitte (B, C) des Massenspektrums von RAFT-PS ${ }_{2 k}-\mathrm{N}^{+}$gemessen in Aceton.

RAFT-PS $\mathrm{k}_{\mathrm{k}}-\mathrm{N}^{+}$hat eine kationische Endgruppe und benötigt kein Metallsalz oder Säure für die lonisierung der Polymerketten in der ESI-MS. Aufgrund der ionischen Endgruppe und des damit verbundenen exzellenten S/R-Verhältnisses von etwa 9000 können bei RAFT$\mathrm{PS}_{2 \mathrm{k}}-\mathrm{N}^{+}$auch Spezies detektiert werden, die nur in Spuren auftreten. So zeigt der Ausschnitt des Massenspektrums in Abbildung 4.8 B neben einer unbekannten einfach geladenen Spezies auch das durch Rekombination entstehende zweifach geladenen Terminierungsprodukt $\mathrm{RR}^{2+}$. Dieses konnte in den Polystyrolen ohne ionische Endgruppe nicht nachgewiesen werden. Da kein Salz zugegeben wurde und die Affinität von Natriumionen für PS gering ist, konnte die Spezies ZI ohne ionische R-Gruppe nicht detektiert werden. Abbildung $4.8 \mathrm{C}$ zeigt einen weiteren Ausschnitt des Massenspektrum bei höheren $\mathrm{m} / \mathrm{z}$. In diesem Bereich werden zwei- und dreifach geladene Spezies nachgewiesen, die nicht nur Natriumaddukte entstehen. Stattdessen sind diese Signale auf "Cluster" zweier oder dreier Polymerketten der Spezies $Z^{+}$, also $2 Z R^{2+}$ bzw. 3ZR ${ }^{3+}$, zurückzuführen. Möglich ist, dass die zwei oder drei Polymerketten über $\pi-\pi$ Wechselwirkungen der Phenylringe „Polymercluster“ bilden.

Fazit

In diesem Abschnitt wurde gezeigt, dass die mittels ESI-MS detektierten Endgruppen zumeist unabhängig vom Polymer und der Molmassen sind. Mit steigender Molmassen neh- 
men bei PMA jedoch die Anzahl der Ladungen zu, wohingegen bei PS nur einfach geladenen Spezies auftreten. PS-Ketten werden auch durch Addukte von $\mathrm{Na}(\mathrm{Nal})$ und $\mathrm{Na}(\mathrm{Nal})_{2}$ ionisiert. Zudem wurde gezeigt, dass eine ionische Endgruppe das S/R-Verhältnis erhöht und daher zur Analyse von Spezies verwendet werden kann, die nur in Spuren auftreten. 


\subsubsection{Einfluss der Natriumkonzentration}

Bei Lösungen der RAFT-Polymere RAFT-PS ${ }_{3 k}-\mathrm{COOH}$ und RAFT-PMA 4 k- $\mathrm{COOH}$ ohne ionische Endgruppe wurde der Einfluss der Nal-Konzentrationen auf das S/R-Verhältnis, die Anzahl der Ladungen und die auftretenden Spezies untersucht.

Die Abbildungen 4.9 A-D zeigen die aufgenommenen Massenspektren von RAFT-PS ${ }_{3 k^{-}}$ $\mathrm{COOH}$ mit 2 bis 20 Äquivalenten Nal. Die Intensitäten der Signale der PS-Ketten im Massenspektrum nehmen mit Zunahme der Nal-Konzentration bis 10 Äquivalente zu (Abb. 4.9 A-C). Tabelle 4.7 zeigt zudem, dass auch das S/R-Verhältnis in diesem Bereich mit der Nal-Konzentration zunimmt, jedoch weniger stark, da das Rauschen stärker ansteigt als die Peakintensitäten. Bei Erhöhung der Nal-Konzentration auf 15 und 20 Äquivalente sinkt sowohl die Intensität am Peakmaximum als auch das S/R-Verhältnis wieder. Ursache dafür sind womöglich die ab 10 Äquivalente $\mathrm{Nal}$ auftretenden $\mathrm{Na}(\mathrm{Nal}){ }_{\mathrm{x}}{ }^{+}-$Cluster (Abbildungen 4.9 $C$ und D). Je höher die Konzentration von Nal, desto wahrscheinlicher wird die Clusterbildung von $\mathrm{Na}^{+}$mit Nal gegenüber der Adduktbildung mit den Polymerketten.
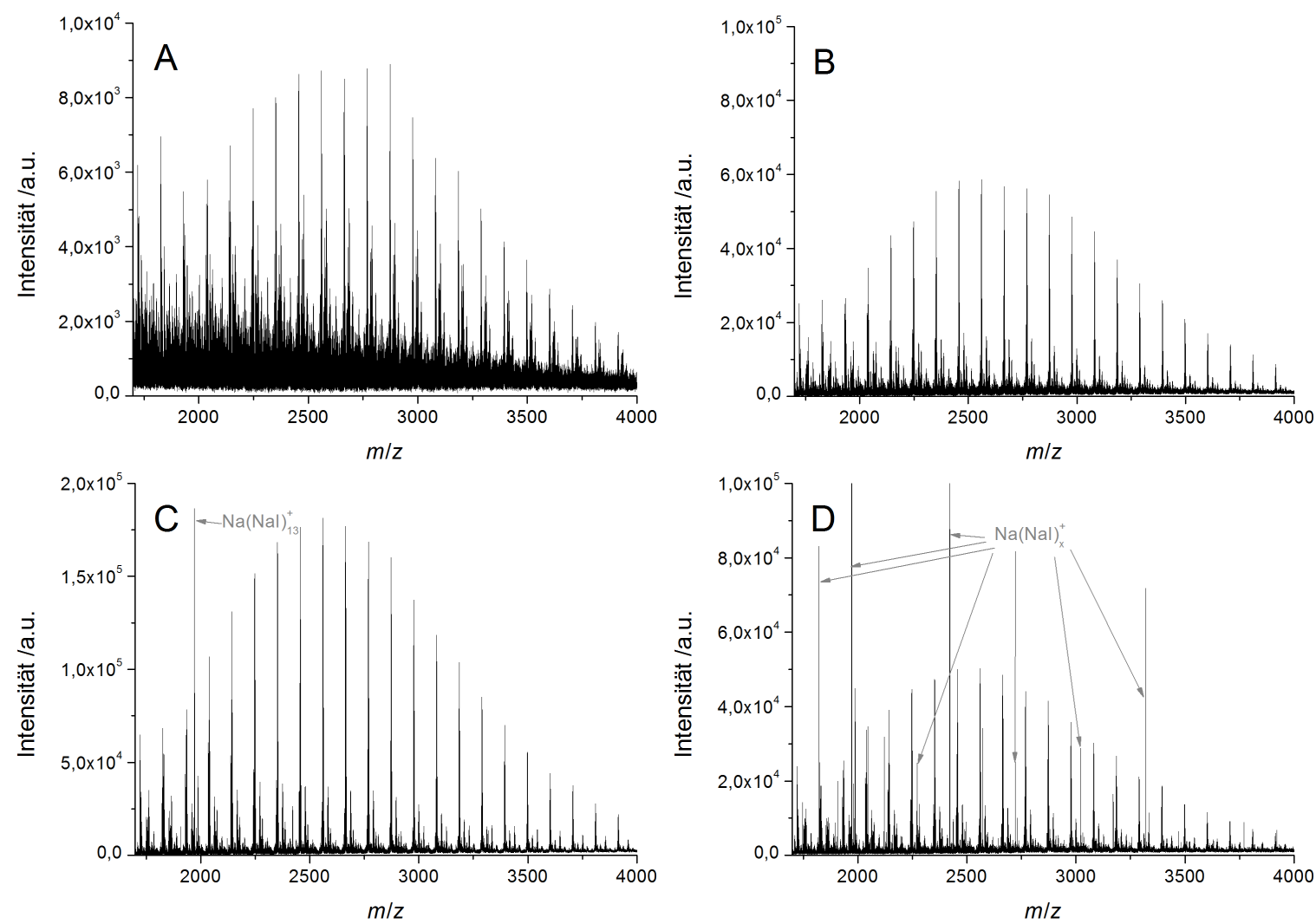

Abb. 4.9. Die ESI-Massenspektren von RAFT-PS ${ }_{3 k}-\mathrm{COOH}$ gemessen in Aceton mit 2 (A), 5 (B), 10 (C) und 20 (D) Äquivalenten an Nal in der Lösung. Die Spektren wurden mit einer Kapillarspannung von $4,5 \mathrm{kV}$ aufgenommen. 

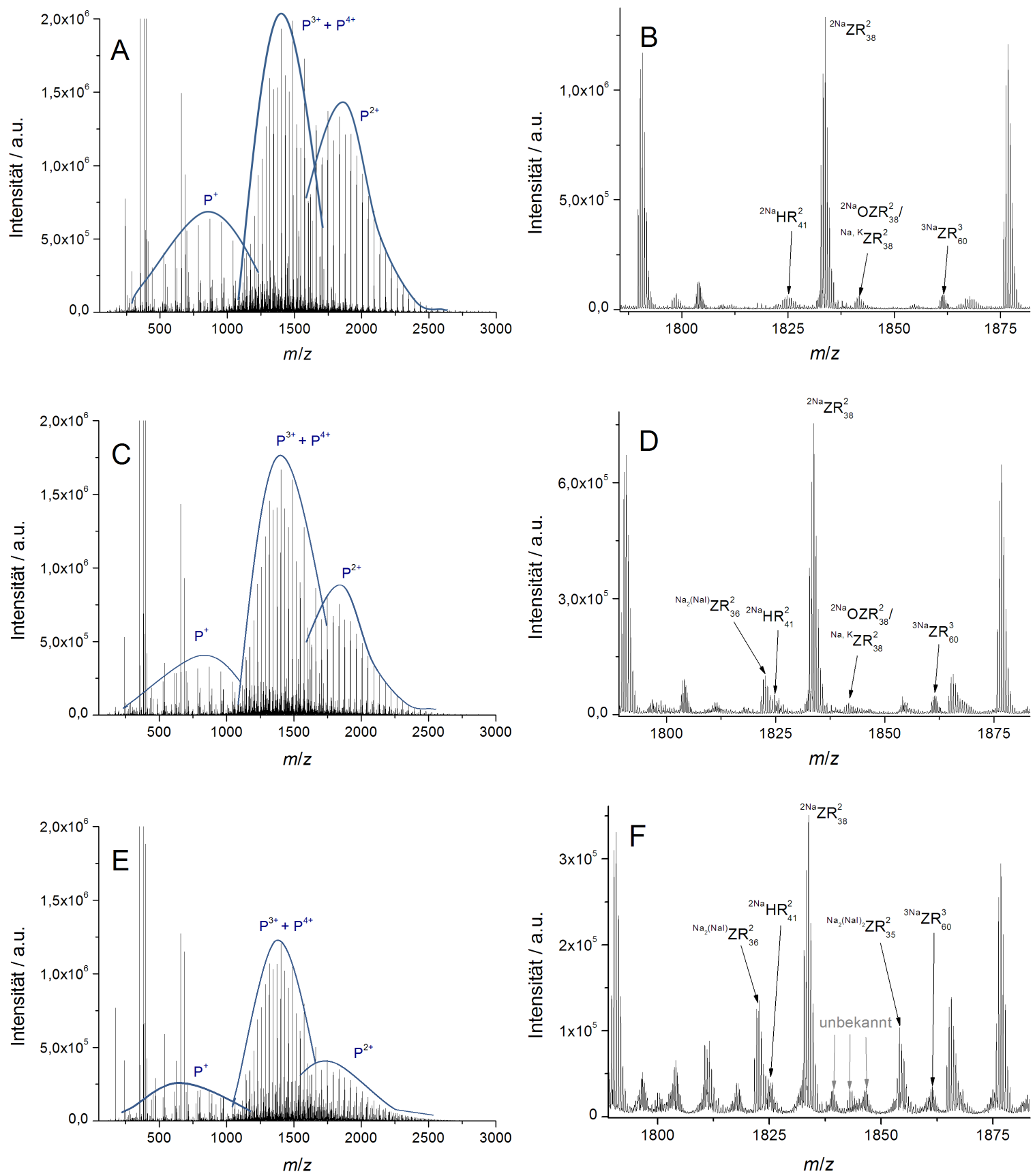

Abb. 4.10. Die ESI-Massenspektren von RAFT-PMA 4 - $-\mathrm{COOH}$ gemessen in Aceton mit 2 (A, B), $5(\mathrm{C}, \mathrm{D})$ und $10(\mathrm{E}, \mathrm{F})$ äquivalenten an $\mathrm{Nal}$ in der Lösung. Die Abbildungen in der rechten Spalte zeigen jeweils einen Ausschnitt der Abbildungen aus der linken Spalte.

Auch bei RAFT-PMA $4 \mathrm{k}-\mathrm{COOH}$ wurde der Einfluss der Nal-Konzentration untersucht. Die Abbildungen $4.10 \mathrm{~A}, \mathrm{C}$ und $\mathrm{E}$ zeigen die Massenspektren aufgenommen mit 2, 5 und 10 Äquivalenten Nal. Im Gegensatz zu der PS-Probe sinken mit zunehmender NalKonzentration sowohl die absoluten Signalintensitäten als auch der relative Anteil der einfach und zweifach geladenen Spezies leicht gegenüber den drei- und vierfach geladenen 
Tab. 4.7. S/R-Verhältnis und die absoluten Signalintensitäten von RAFT-PS ${ }_{3 k}-\mathrm{COOH}$ und RAFT$\mathrm{PMA}_{4 \mathrm{k}}-\mathrm{COOH}$ am Peakmaximum $\left(M_{\mathrm{p}}\right)$ in Abhängigkeit von der Nal-Konzentration (vergleiche Abb. 4.9 und 4.10.

\begin{tabular}{cccccc}
\hline Polymer & Nal-Äquivalente & 2 & 5 & 10 & 20 \\
\hline $\mathrm{PS}_{3 \mathrm{k}}$ & S/R-Verhältnis & 8 & 24 & 53 & 22 \\
& Intensität / (1000 a.u.) & 9 & 59 & 180 & 50 \\
\hline $\mathrm{PMA}_{4 \mathrm{k}}$ & S/R-Verhältnis & 87 & 62 & 45 & - \\
& Intensität / (1000 a.u.) & 1900 & 1700 & 1200 & - \\
\hline
\end{tabular}

Spezies. Zudem sinkt auch das S/R-Verhältnis mit zunehmender Nal-Konzentration. Anders als bei RAFT-PS ${ }_{3 k}-\mathrm{COOH}$ treten bei der PMA-Probe mit 10 Äquivalenten auch keinerlei $\mathrm{Na}(\mathrm{Nal})_{\mathrm{x}}{ }^{+}$-Cluster auf. Eine mögliche Erklärung für diese Beobachtung ist, dass PMA eine höhere Affinität zu Natriumionen hat. Die Zugabe von 2 Äquivalenten Nal reicht demnach aus, um die meisten Ketten durch Natriumadduktbildung zu ionisieren. Die weitere Zugabe von Nal erhöht lediglich den Anteil an dreifach und vierfach geladenen Ketten und erschwert so die Interpretation des Spektrums.

Durch die erhöhte Natriumkonzentration werden bei PMA nicht nur mehr drei- und vierfach

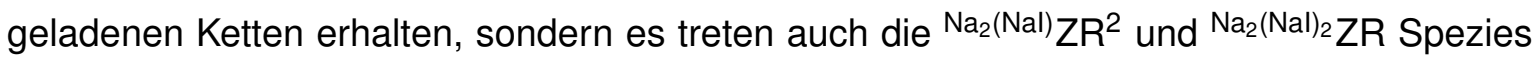
auf (Abb. 4.10 D bzw. F und Tabelle B8). Diese sind durch zwei Natriumionen ionisiert und zusätzlich sind ein bzw. zwei Nal an der Kette angelagert. Ähnliche $\mathrm{Na}(\mathrm{Nal})$-Polymeraddukte wurden bereits bei RAFT-PS ${ }_{2 \mathrm{k} / 3 \mathrm{k}}-\mathrm{COOH}$ im Abschnitt 4.4.1 detektiert. Bei der Verwendung von 10 Äquivalenten Nal treten zudem noch drei unbekannte Spezies auf (Abb. 4.10 F).

Zusammenfassend ist festzustellen, dass mit Erhöhung der Nal-Konzentration die Signalintensitäten bei PMA abnehmen und bei PS erst steigen und an einer bestimmten Konzentration wieder abnehmen. Aufgrund der $\mathrm{Na}(\mathrm{Nal})$-Cluster und -Polymer-Clusteraddukte muss die Konzentration von Nal für jede Probe angepasst werden. 


\subsubsection{Einfluss der Kapillarspannung}

Anhand von RAFT-PMA $1 \mathrm{k}^{-} \mathrm{N}^{+}$wird der Einfluss der Kapillarspannung auf mögliche Fragmentierungsprodukte untersucht. Die Abbildungen 4.11 A-D zeigen die Massenspektren des Polymers bei der Verwendung einer Kapillarspannung von 2,5 bis $5 \mathrm{kV}$. Bei der Erhöhung der Spannung von 2,5 auf $3 \mathrm{kV}$ steigt die Intensität der Signale und das S/R-Verhältnis an (Tabelle 4.8), da eine höhere Spannung auch eine höhere Stromstärke und somit mehr freie Ladungsträger erzeugt. Bei diesen beiden niedrigen Spannungen treten nur die aus Abschnitt 4.4.1 bekannten Spezies auf.

Wird die Spannung weiter erhöht, nehmen die Signalintensitäten und das S/R-Verhältnis ab. Die HR-Spezies tritt zwar bereits bei 2,5 und $3 \mathrm{kV}$ auf, allerdings bei geringeren $\mathrm{m} / \mathrm{z}$ Werten und sie ist weniger häufig als bei der 4 und $5 \mathrm{kV}$ Messung. Von 4 auf $5 \mathrm{kV}$ nimmt der Anteil der HR gegenüber der ZR Spezies nochmals zu. Dies und die Differenz der Peakmaxima der ZR und HR Spezies legt nahe, dass bei höherer Kapillarspannung die RAFT-Gruppe vom Polymer abgespalten wird. Reaktionen von Analyten und Lösungsmittel an der Kapillare sind durchaus bereits in der Literatur bekannt. Die Oxidation von Thiolen zu Disulfiden an der Kapillarelektrode beispielsweise in der Literatur beschrieben. ${ }^{178}$ Dabei findet die
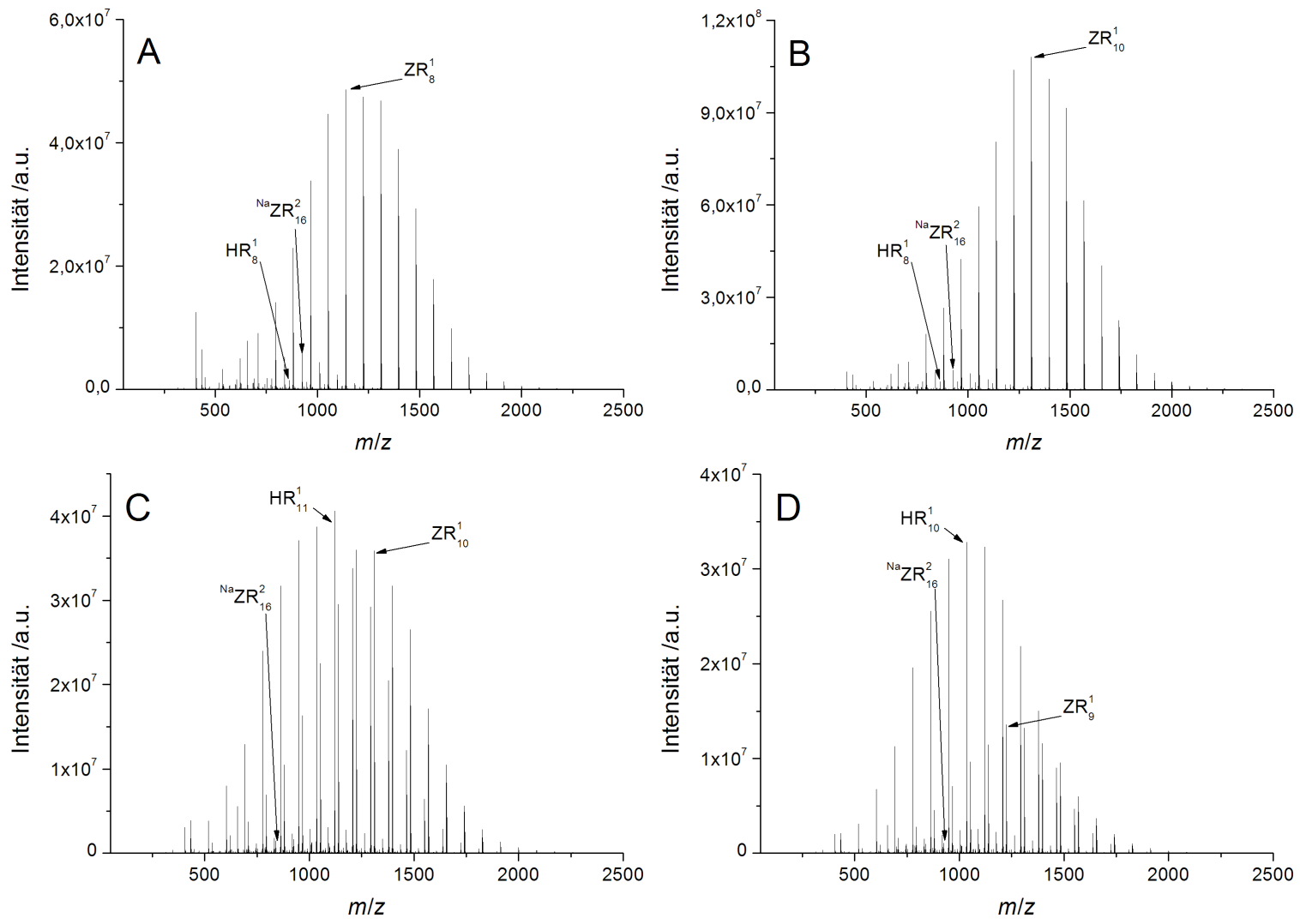

Abb. 4.11. Das komplette ESI-Massenspektrum von RAFT-PMA ${ }_{1 k}-\mathrm{N}^{+}$bei Verwendung einer Kapillarspannung von 2,5 (A), 3 (B), 4 (C) und 5 kV (D) gemessen in Aceton. Die markierten Peaks entsprechen den Peakmaximum $M_{\mathrm{p}}$ der jeweiligen Spezies. 


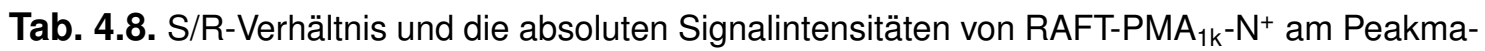
ximum $\left(M_{\mathrm{p}}\right)$ in Abhängigkeit von der Kapillarspannung.

\begin{tabular}{cllll}
\hline Kapillarspannung / kV & 2,5 & 3 & 4 & 5 \\
\hline S/R-Verhältnis & 3000 & 10000 & 1500 & 1300 \\
Signalintensität / $\left(10^{5}\right.$ a.u. $)$ & 490 & 1100 & 400 & 330 \\
\hline
\end{tabular}

Oxidation vor allem bei höheren Strömen und entsprechend des Ohmschen Gesetztes bei höheren Spannungen statt. [157/179|180] Oxidationsprodukte der Dithiobenzoate wurden auch ohne den Einsatz von Oxidationsmitteln in der ESI-MS-Analyse nachgewiesen und die Oxidationsprodukte der Trithiocarbonate (Spezies OZR und ZOR) konnten in Abschnitt 4.4.1 detektiert werden. 164-166

Die Vergrößerungen der Massenspektren bei einer Kapillarspannung von 3 und $5 \mathrm{kV}$ sind in Abbildung 4.12 $A$ und $B$ gezeigt. Neben der vermehrten Bildung von HR treten aufgrund der höheren Kapillarspannung noch weitere neue Spezies auf, von denen zwei Spezies allerdings keine Struktur zugeordnet werden konnte. Es werden auch Polymerketten detektiert, die statt durch die RAFT-Gruppe durch ein Hydroperoxid oder ein Aceton terminiert sind (HOOR oder AcR Spezies). Die geringen Abweichungen der gefundenen $\mathrm{m} / \mathrm{z}$-Werte gegenüber den berechneten in der Tabelle $\mathrm{B} 9 \mathrm{im}$ Anhang bestätigen die gefundenen Strukturen HOOR und AcR. Das Auftreten der vermehrten HR und das Auftreten dieser beiden Spezies könnte durch eine homolytische Spaltung der C-S-Bindung zwischen Polymerkette und RAFT-Gruppe erklärt werden (Schema 4.6). Das entstehende polymere Radikal kann ein Wasserstoffatom von einem H-Donor abstrahieren und so die Spezies HR bilden. Fungiert Aceton als $\mathrm{H}$-Donor, so entsteht dabei ein Acetonradikal. Die Bildung eines Acetonradikals durch Wasserstoffabstraktion eines anderen Radikals ist literaturbekannt. 181-183] Ein Acetonradikal kann auch mit einem polymeren Radikal rekombinieren und so die AcR Spezies bilden. Die hydroperoxidterminierte Spezies HOOR entsteht durch die Reaktion eines polymeren Radikals mit Sauerstoff und anschließender H-Abstraktion.
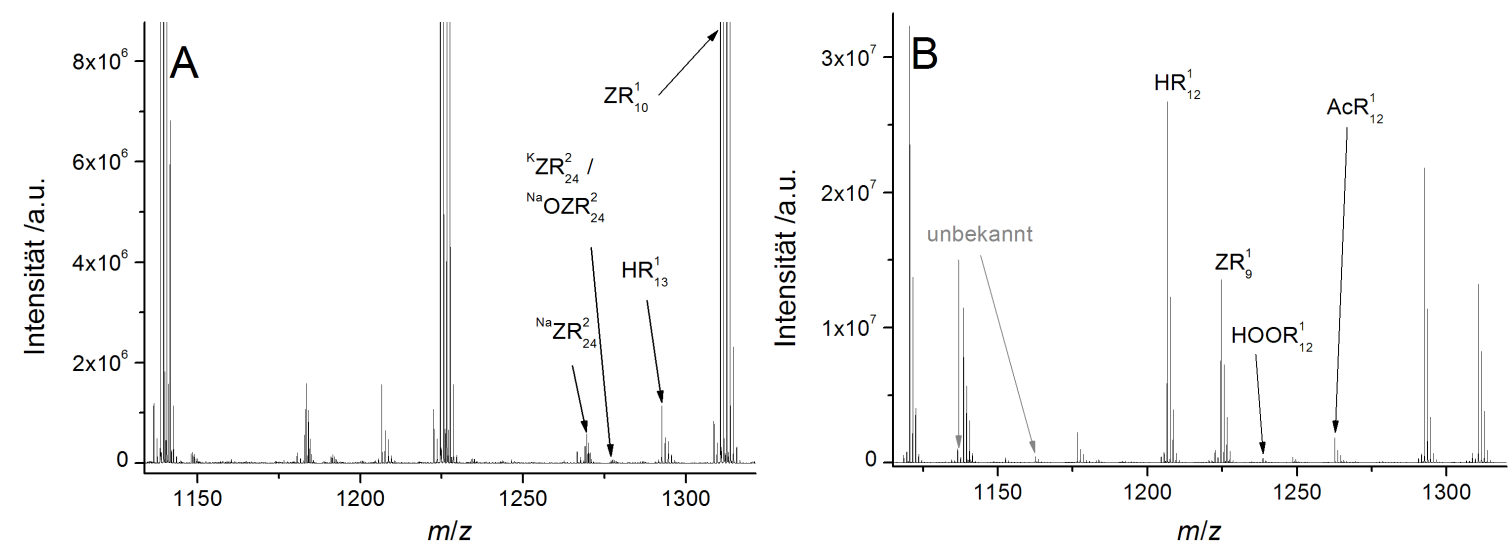

Abb. 4.12. Ausschnitte des ESI-Massenspektrums von RAFT-PMA $\mathrm{Ak}^{-} \mathrm{N}^{+}$bei Verwendung einer Kapillarspannung von $3 \mathrm{kV}(\mathrm{A})$ und $5 \mathrm{kV}(\mathrm{B})$ gemessen in Aceton. 
Zur Überprüfung dieser Hypothese wurde die Probe RAFT-PMA $\mathrm{k}^{-\mathrm{N}^{+}}$nochmals in einem Lösungsmittelgemisch aus $\mathrm{MeOH}$ und DCM sowie mit einer Kapillarspannung von $3 \mathrm{kV}$ und $5 \mathrm{kV}$ gemessen. Die Abbildungen 4.13 A und B zeigen Ausschnitte der Messung im Gemisch aus $\mathrm{MeOH}$ und DCM bei einer Kapillarspannung von $3 \mathrm{kV}$ sowie $5 \mathrm{kV}$. Diese Messung wurde zu einem späteren Zeitpunkt durchgeführt, als die Detektorsensitivität bereits wesentlich geringer war. Daher sind die Signalintensitäten geringer als in Abbildung 4.11. Gegenüber der Messung in Aceton (Abb. 4.12) fällt auf, dass bei $3 \mathrm{kV}$ die OZR Spezies nicht auftritt. Bei $5 \mathrm{kV}$ wird zudem im Gemisch aus MeOH und DCM weder die HOOR noch die AcR Spezies detektiert, was den möglichen Mechanismus für deren Bildung in Aceton nach Schema 4.6 unterstützt. Das Lösungsmittelgemisch von $\mathrm{MeOH}$ und DCM produziert sowohl bei $3 \mathrm{kV}$, als auch bei $5 \mathrm{kV}$ weniger Endgruppen (Spezies) als die Messungen in Aceton und der Anteil der HR Spezies im Massenspektrum sinkt ebenfalls. In zukünftigen Messungen ist daher das Lösungsmittelgemisch aus $\mathrm{MeOH}$ und DCM dem Aceton als Lösungsmittel vorzuziehen

Es konnte gezeigt werden, dass die Kapillarspannung gerade bei labilen Gruppen wie Trithiocarbonaten zur Fragmentierung führen kann. Daher muss die Kapillarspannung für ein möglichst hohes S/R-Verhältnis und möglichst wenig Fragmentierung optimiert werden. Eine Spannung von $3 \mathrm{kV}$ hat sich hier als das Optimum herausgestellt.

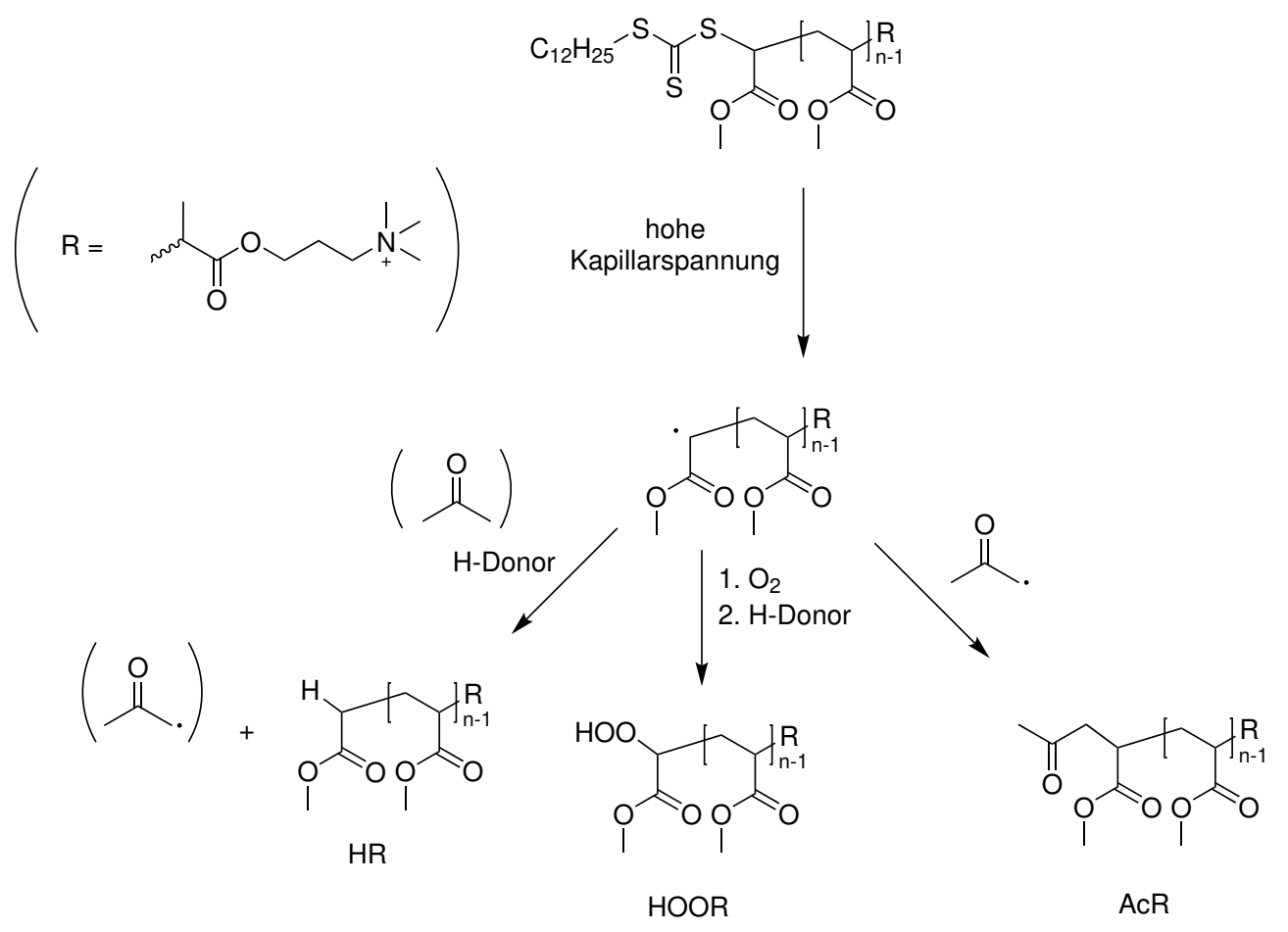

Schema 4.6. Möglicher Mechanismus, der die Entstehung der Spezies HR, HOOR und AcR erklärt. 

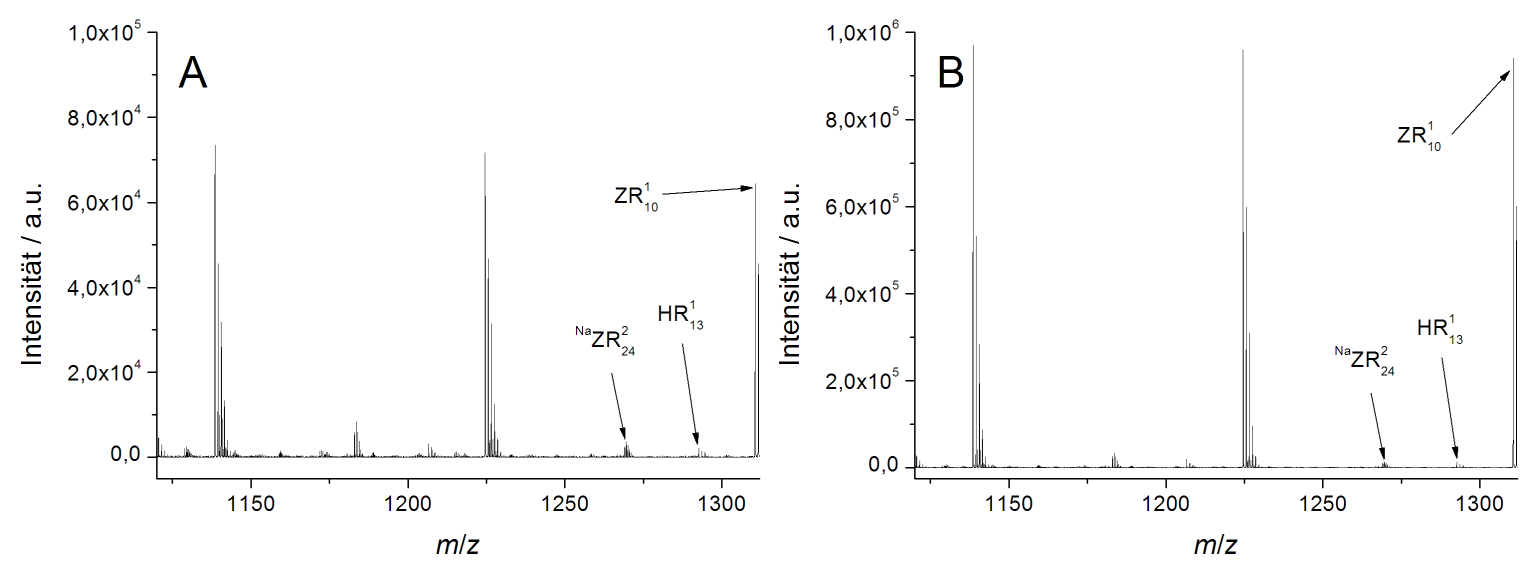

Abb. 4.13. Ausschnitte des ESI-Massenspektrums von RAFT-PMA $A_{1 k}-N^{+}$bei Verwendung einer Kapillarspannung von $3 \mathrm{kV}(\mathrm{A})$ und $5 \mathrm{kV}(\mathrm{B})$ gemessen in MeOH:DCM (1:1). 


\subsubsection{Einfluss der Flussrate}

Der Einfluss der Flussrate der Polymerlösung auf die ESI-Massenspektren wurde für Polymere mit terminaler Ammoniumgruppe anhand von RAFT-PS ${ }_{2 k}-\mathrm{N}^{+}$und für Polymere ohne terminale Ammoniumgruppe anhand von RAFT-PMA ${ }_{2 k}-\mathrm{COOH}$ untersucht. Standardmäßig wurde eine Flussrate von $300 \mu \mathrm{L} \mathrm{h}^{-1}$ verwendet.

Abbildung 4.14 zeigt die ESI-Massenspektren von RAFT-PMA ${ }_{2 \mathrm{k}}-\mathrm{COOH}$ bei einer Flussrate von 300 (A) 600 (B) und 1200 (C) $\mu \mathrm{L} \mathrm{h}^{-1}$. Es ist zu erkennen, dass mit steigender Flussrate der relative Anteil der doppelt geladenen Ketten gegenüber den einfach geladenen Ketten abnimmt. Die Verwendung einer höheren Flussrate kann daher die Interpretation eines Massenspektrums erleichtern Eine mögliche Erklärung dafür ist, dass der Strom nahezu unabhängig von der Flussrate ist. ${ }^{[15 / 184}$ Dadurch ist die Anzahl von Ladungsträger (Natriumionen) ebenfalls nahezu unabhängig von der Flussrate, aber es stehen pro Zeit mehr Polymerketten zur Verfügung. Somit werden bei höherer Flussrate bevorzugt einfach geladene Ketten gebildet.

Tabelle 4.9 zeigt, dass bei RAFT-PMA ${ }_{2 \mathrm{k}}-\mathrm{COOH}$ sowohl die Intensitäten an $M_{\mathrm{p}}$ als auch das S/R-Verhältnis mit zunehmender Flussrate steigt. Massenspektrometer werden als massenflussempfindliche Detektoren beschrieben, in denen die Signalintensitäten proportional
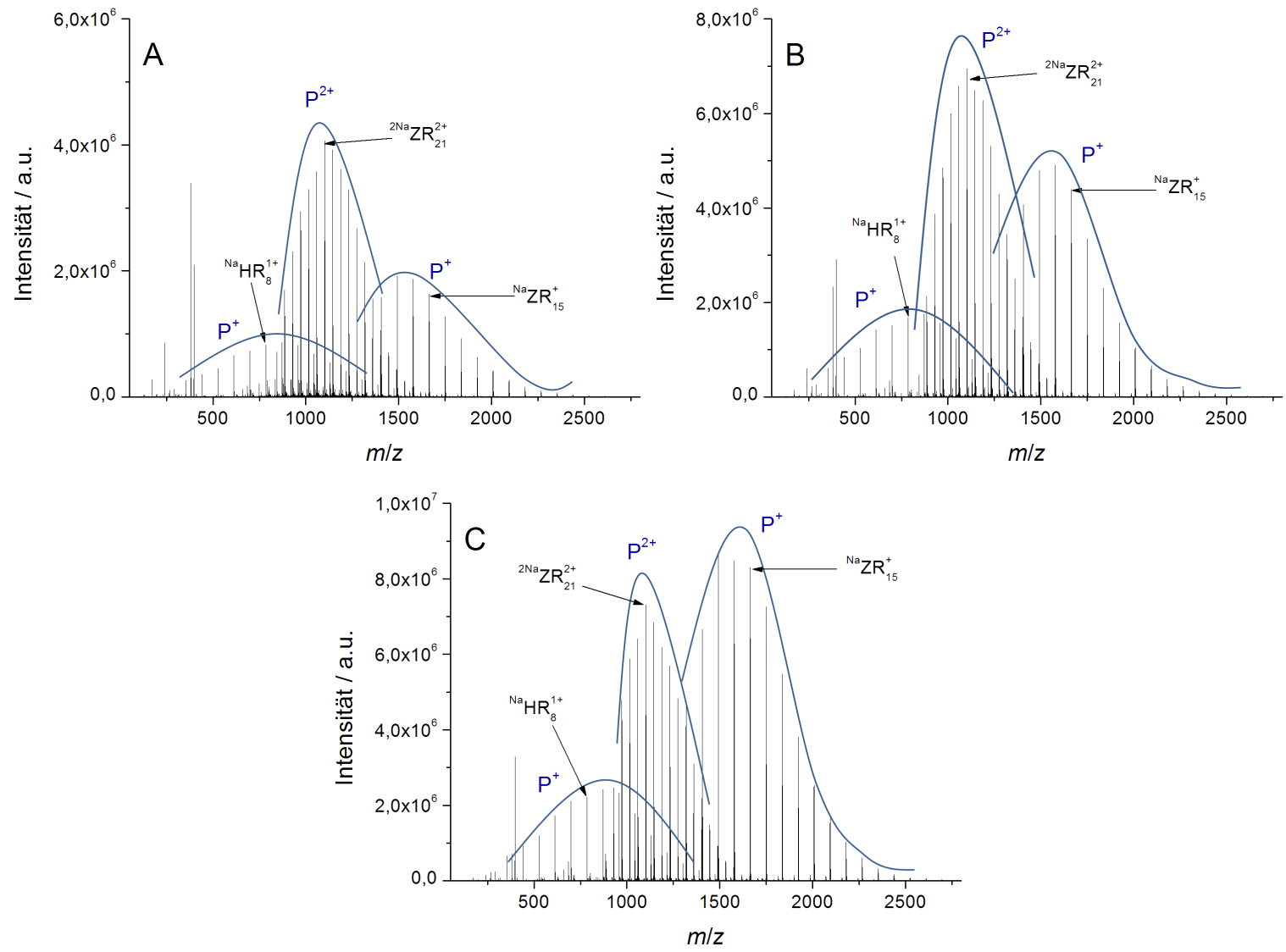

Abb. 4.14. Die ESI-Massenspektren von RAFT-PMA ${ }_{2 k}-\mathrm{COOH}$ bei einer Flussrate von 300 (A) 600 (B) und 1200 (C) $\mu \mathrm{L} \mathrm{h}^{-1}$. 
zur Menge des Analyten ist, die pro Zeit auf den Detektor trifft. ${ }^{185[186]}$ Mit zunehmender Flussrate steigt auch die Menge des Analyten pro Zeit.

Auch bei RAFT-PS ${ }_{2 k}-\mathrm{N}^{+}$steigt mit zunehmender Flussrate das S/R-Verhältnis und die Intensität der Peaks an (Abbildung 4.15 A und B sowie Tabelle 4.9). Des Weiteren zeigen die Abbildungen 4.15 A und $B$ sowie $C$ und $D$, dass der Anteil der detektierten HR und der unbekannten Spezies mit steigender Flussrate abnimmt. Eine mögliche Erklärung für diese Beobachtung wäre, dass diese Spezies durch Reaktionen während des ESI-Prozesses entstehen und dass durch die höhere Flussrate die Zeit in der Kapillare für diese Reaktion verkürzt wird.
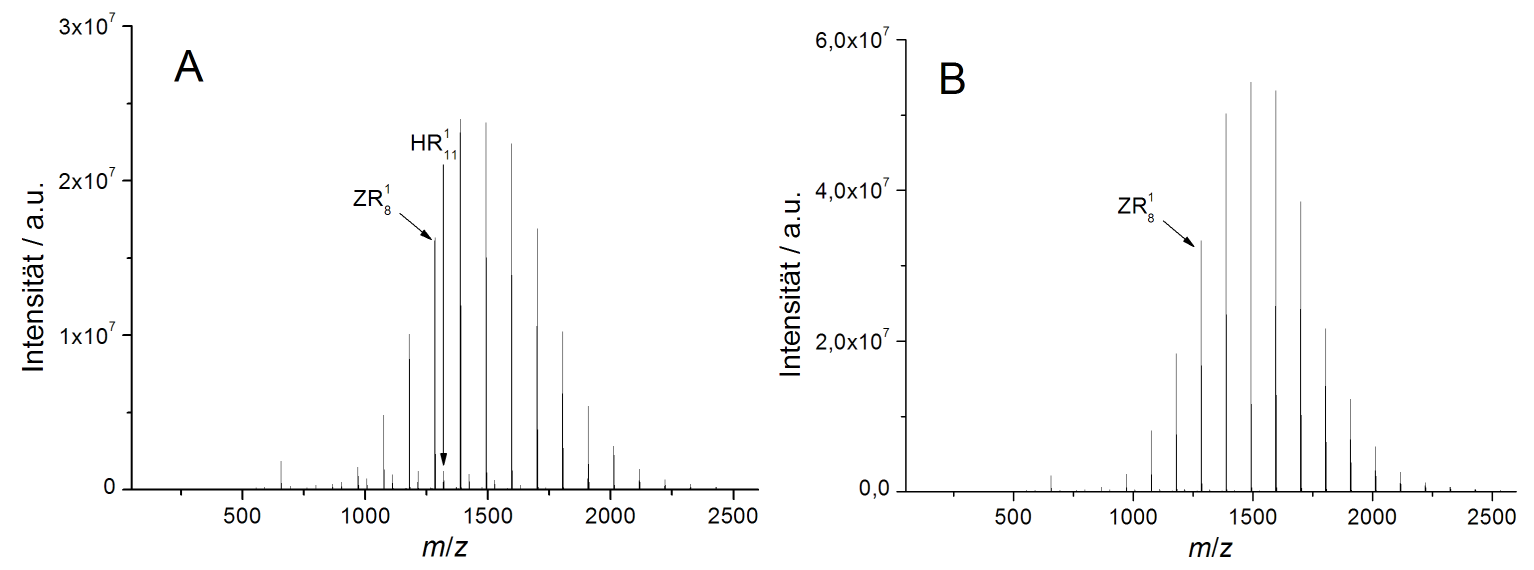

Abb. 4.15. Die kompletten ESI-Massenspektren und Ausschnitte der Spektren von RAFT-PS $2 \mathrm{k}^{-}$ $\mathrm{N}^{+}$bei einer Flussrate von $300(A)$ und $600 \mu \mathrm{L} \mathrm{h}^{-1}(\mathrm{~B})$.

Tab. 4.9. $S / R$-Verhältnis und die absoluten Signalintensitäten von RAFT-PMA $A_{2 k}-C O O H$ und RAFT-PS ${ }_{2 \mathrm{k}}-\mathrm{N}^{+}$am Peakmaximum $\left(M_{\mathrm{p}}\right)$ in Abhängigkeit von der Flussrate.

\begin{tabular}{c|cccc}
\hline Polymer & Flussrate $/ \mu \mathrm{L} \mathrm{h}^{-1}$ & 300 & 600 & 1200 \\
\hline RAFT-PMA $_{2 \mathrm{k}}-\mathrm{COOH}$ & S/R-Verhältnis & 270 & 990 & 1200 \\
& Signalintensität / $\left(10^{5}\right.$ a.u.) & 40 & 69 & 87 \\
\hline RAFT-PS $_{2 \mathrm{k}}-\mathrm{N}^{+}$ & S/R-Verhältnis & 9600 & 36000 & \\
& Signalintensität / $\left(10^{6}\right.$ a.u.) & 24 & 54 & \\
\hline
\end{tabular}




\subsubsection{Einfluss der einfach und zweifach geladenen ionischen Endgruppe}

Der Einfluss der ionischen Endgruppe auf die Spektrenqualität und Sensitivität wurde anhand des direkten Vergleichs von PMA und PS mit und ohne ionische Endgruppe durchgeführt. Die Abbildungen 4.16 A und $B$ zeigen die Massenspektren von RAFT-PMA ${ }_{2 k}-\mathrm{COOH}$ und RAFT-PMA ${ }_{1 k}-\mathrm{N}^{+}$. Bei RAFT-PMA $2 \mathrm{k}-\mathrm{COOH}$ wurden nur zwei Äquivalente Nal zugegeben, da eine höhere Nal-Konzentration das S/R-Verhältnis sowie die Peakintensitäten erniedrigt und vermehrt zu zweifach geladenen Ketten führt (vergleiche Abschnitt 4.4.2).

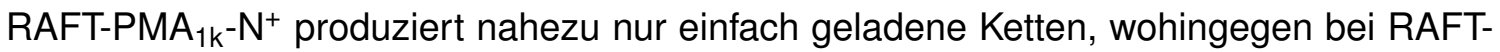
$\mathrm{PMA}_{2 \mathrm{k}}-\mathrm{COOH}$ die Intensität der zweifach geladenen Ketten höher ist als die der einfach geladenen. Der Grund dafür ist, dass zum einen die Molmasse von RAFT-PMA $2 \mathrm{k}-\mathrm{COOH}$ etwas höher als die von RAFT-PMA ${ }_{1 k^{-}} \mathrm{N}^{+}$ist, weshalb zweifach geladenen Ketten aufgrund der höheren Zahl an Bindungsstellen für Natrium bei dieser Probe wahrscheinlicher sind. Zum anderen wurde bei bei RAFT-PMA $1 \mathrm{k}^{-\mathrm{N}^{+}}$kein weiteres Natriumsalz zugegeben, d.h. die Konzentration an Natrium ist geringer. Ein weiterer großer Vorteil der ionischen Endgruppe ist, dass bei RAFT-PMA $1 \mathrm{k}^{-} \mathrm{N}^{+}$die Peakintensitäten und das $\mathrm{S} / \mathrm{R}$-Verhältnis um einen Faktor von 25 gegenüber dem durch Natriumaddukte ionisierten RAFT-PMA $2 \mathrm{k}-\mathrm{COOH}$ erhöht sind (Tabelle 4.10). Dies ist gerade in der Spurenanalyse der Polymere von Vorteil, aber auch bei der Identifikation von Spezies bzw. Endgruppen die nur in geringem Maße auftreten (vergleiche Abschnitt 4.4.1).

PS neigt auch nicht zur Bildung von zweifach geladenen Ketten, weshalb 10 Äquivalente von Nal RAFT-PS ${ }_{2 \mathrm{k}}-\mathrm{COOH}$ zur lonisierung zugegeben wurden (siehe Abschnitt 4.4.2). In den Massenspektren der beiden PS-Proben (Abb. 4.16 $C$ und $D$ ) ist ebenfalls ein deutlicher Unterschied zu sehen. Bei RAFT-PS ${ }_{2 k}-\mathrm{COOH}$ ist das S/R-Verhältnis gering und Hintergrundsignale sind deutlich zu erkennen oder gar intensiver als die Polymersignale. Das S/R-Verhältnis und die Peakintensitäten sind bei der PS-Probe mit der ionischen Endgruppe um etwa den Faktor 140 bzw. 170 höher, obwohl die Molmasse der Probe mit der ionischen Endgruppe sogar etwas größer ist als bei der Probe RAFT-PS ${ }_{2 k}-\mathrm{COOH}$ (siehe Tabelle 4.1 und 4.2). Der Unterschied zwischen Polymer mit ionischer Endgruppe und dem mit Natrium ionisierten Polymer ohne ionische Endgruppe ist bei PS größer als bei PMA,

Tab. 4.10. S/R-Verhältnis und die absoluten Signalintensitäten von PMA- und PS-Proben mit und ohne ionische Endgruppe am Peakmaximum $\left(M_{\mathrm{p}}\right)$.

\begin{tabular}{|c|c|c|}
\hline Polymer & S/R-Verhältnis & Signalintensitäten / (10 $10^{5}$ a.u. $)$ \\
\hline RAFT-PMA ${ }_{2 \mathrm{k}}-\mathrm{COOH}$ & 400 & 40 \\
\hline RAFT-PMA $1 \mathrm{k}^{-\mathrm{N}^{+}}$ & 10000 & 1100 \\
\hline 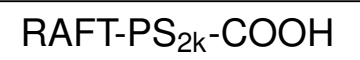 & 70 & 1,4 \\
\hline RAFT-PS ${ }_{2 \mathrm{k}}-\mathrm{N}^{+}$ & 9600 & 240 \\
\hline
\end{tabular}



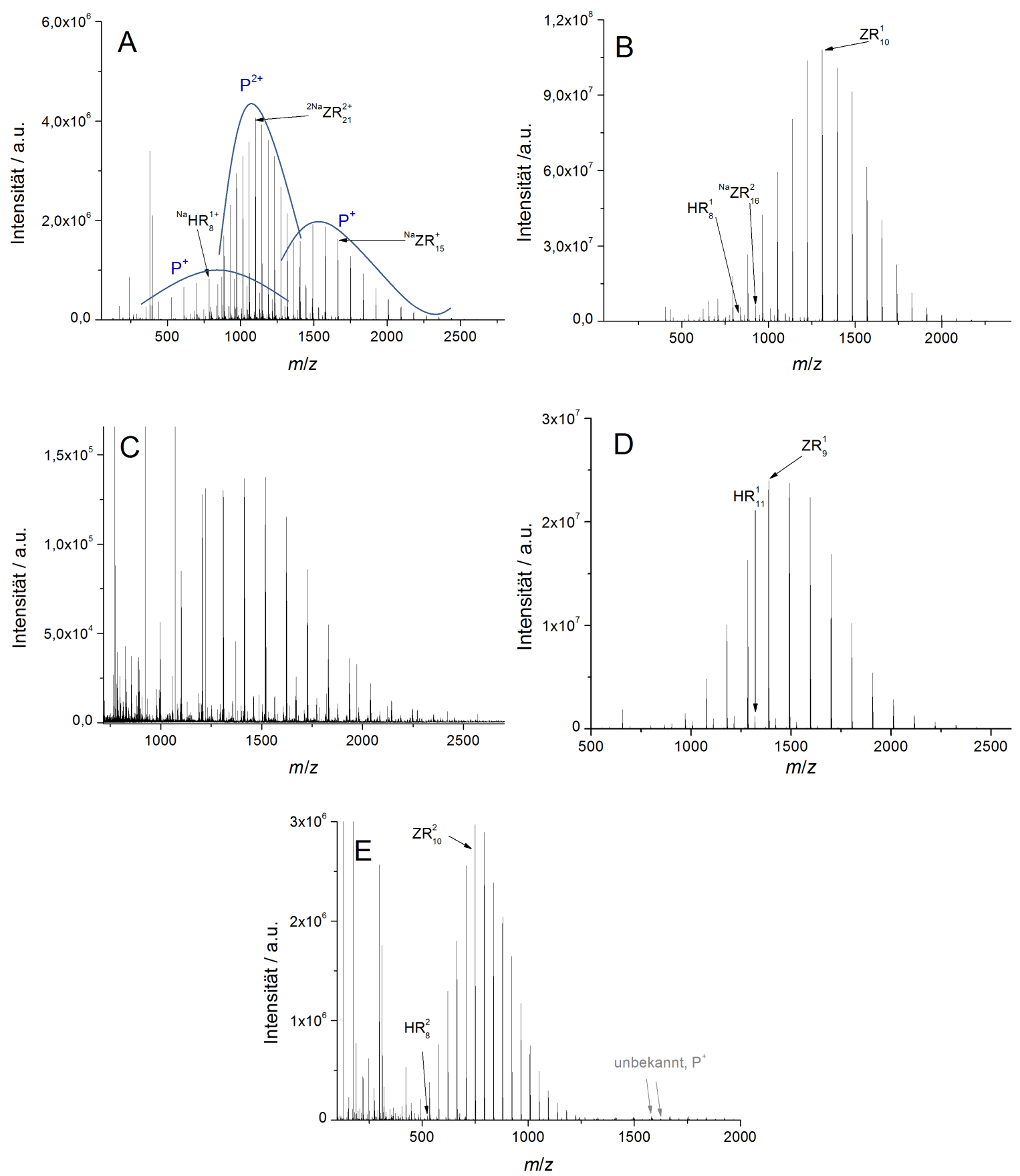

Abb. 4.16. Das ESI-Massenspektrum von RAFT-PMA $A_{2 k}-\mathrm{COOH}$ mit zwei Äquivalenten Nal (A), RAFT-PMA $1 \mathrm{k}^{-} \mathrm{N}^{+}(\mathrm{B}), \mathrm{RAFT}-\mathrm{PS}_{2 \mathrm{k}}-\mathrm{COOH}$ mit 10 Äquivalenten $\mathrm{Nal}(\mathrm{C})$, RAFT-PS ${ }_{1,5 \mathrm{k}}-\mathrm{N}^{+}$(D) und RAFT-PMA $2 \mathrm{k}^{-} \mathrm{N}^{2+}(\mathrm{E})$. Bei allen Messungen betrug die Polymerkonzentration $50 \mu \mathrm{M}$, die Flussrate $300 \mu \mathrm{L} \mathrm{h}^{-1}$ und die Kapillarspannung $3 \mathrm{kV}$. 
da die Bindung von Natrium an PMA stärker ist als an PS. Der Vergleich von RAFT-PS $2 \mathrm{k}^{-\mathrm{N}^{+}}$ mit dem mittels Adduktbildung durch Silber oder Kupfer ionisierten RAFT-PS ${ }_{2 k}-\mathrm{COOH}$ wurde nicht durchgeführt, da diese Übergangsmetalle zur Abspaltung der Trithiocarbonatgruppe führen. $174-176$

Von Interesse ist nicht nur die Erhöhung der Sensitivität, sondern auch den Ladungszustand gezielt zu kontrollieren. Abbildung 4.16 E zeigt, dass dies bei RAFT-PMA $1,5 \mathrm{k}-\mathrm{N}^{2+}$ möglich ist. Durch die zwei Ammoniumgruppen in der Endgruppe entstehen nahezu ausschließlich zweifach geladene Polymerketten, obwohl bei RAFT-PMA ${ }_{2 k}-\mathrm{COOH}$ - sogar mit einem etwas höheren Polymerisationsgrad - noch viele einfach geladene Ketten auftreten (Abb.4.16A). Erst bei höheren $\mathrm{m} / \mathrm{z}$-Werten werden Spuren von unbekannten, einfach geladenen Polymerketten detektiert. 


\subsubsection{Einfluss der Stoßenergie auf mehrfach geladenen Polymerketten in der ESI-MS}

Das verwendete ESI-Massenspektrometer ist mit einer Kollisionszelle ausgestattet. In dieser wird den lonen durch Stöße Energie zugeführt, wobei die Stoßenergie über die Spannung eingestellt werden kann. Die Stoßenergie kann zur Fragmentierung, aber auch zum Entclustern verwendet werden. Die Stoßenergie beeinflusst die Massenspektren in ähnlicher Weise wie die cone voltage von ESI-Massenspektrometern älterer Bauweise. 187-190 In diesem Abschnitt wird der Einfluss der Stoßenergie in der ESI-Massenspektrometrie von mehrfach geladenen Polymeren mit und ohne ionische Endgruppen untersucht.

\section{Einfluss der Stoßenergie auf Polymerketten ohne ionische Endgruppe}

Abbildungen 4.17 A und $B$ zeigen die ESI-Massenspektren von RAFT-PMA ${ }_{2 k}-\mathrm{COOH}$ ohne Stoßenergie $(A)$ und mit einer Stoßenergie von $50 \mathrm{~V}(\mathrm{~B})$. Mit zehn Äquivalenten wurde eine höhere Konzentration von Nal gegenüber Abschnitt 4.4.1 (Abb. 4.4) verwendet, weshalb ohne Stoßenergie auch via Na-Nal-Cluster ionisierte Spezies auftreten. Bereits bei einer Stoßenergie von $50 \mathrm{~V}$ sind die PMA-Ketten vollständig entclustert und es treten nur noch mittels Natriumaddukte ionisierte Ketten auf. Zusätzlich wird der relative Anteil an einfach geladenen Ketten durch die Stoßenergie und das Entclustern erhöht. Jedoch wird vermehrt die Spezies ${ }^{2 N a Z R^{1}}$ detektiert, bei der ein Natrium an die deprotonierte endständige Carboxylgruppe gebunden ist. Zudem entstehen unterhalb von $1000 \mathrm{~m} / \mathrm{z}$ auch einfach geladene Fragmente unbekannter Struktur und die Signale werden leicht zu höheren $\mathrm{m} / \mathrm{z}$-Werten verschoben.

Die Abbildungen 4.17 C und D zeigen den Einfluss des Stoßenergie auf RAFT-PMA $4 \mathrm{k}^{-}$ $\mathrm{COOH}$ in der ESI-MS. Bei einer Stoßenergie von $70 \mathrm{~V}$ steigt der Anteil von zweifach geladenen gegenüber den dreifach geladenen Ketten. Allerdings treten aber auch viele einfach geladene Kettenfragmente unbekannter Struktur auf. Wie bereits erwähnt kann die Zuführung von Stoßenergie auch zur Fragmentatierung der lonen führen, da durch die Stöße ein Teil der kinetischen Energie in innere Energie umgewandelt wird. 189-192] Eine weitere Erhöhung der Stoßenergie führte lediglich zu einer starken Zunahme der Fragmentionen und einer Abnahme der zweifach und dreifach geladenen Ketten.

Auch bei RAFT-PMA ${ }_{7 k}-\mathrm{COOH}$ wurde der Einfluss der Stoßenergie einmal ohne Zugabe von Nal und einmal mit zehn Äquivalenten Nal untersucht. Die Massenspektren sind in den Abbildungen A8 $A$ bis $D$ im Anhang gezeigt. Auch bei diesem Polymer tritt nur eine leichte Erniedrigung des mittleren Ladungszustandes mit zunehmender Stoßenergie auf und es entstehen vermehrt einfach geladene, unbekannte Fragmente. 

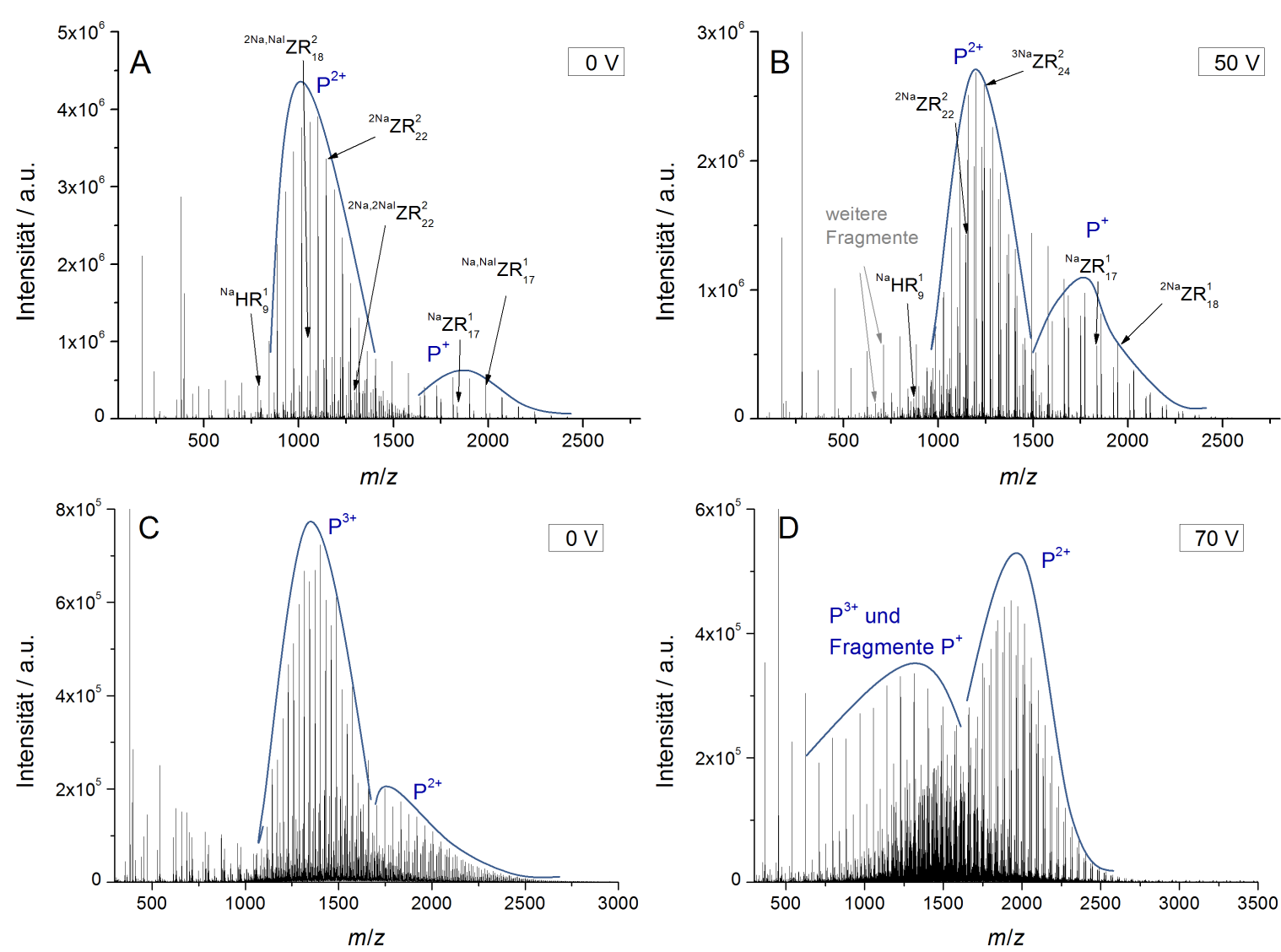

Abb. 4.17. Das ESI-Massenspektrum einer $50 \mu \mathrm{M}$ Lösung von RAFT-PMA $A_{2 k}-\mathrm{COOH}(\mathrm{A}, \mathrm{B})$ und RAFT-PMA $4 \mathrm{k}-\mathrm{COOH}(\mathrm{C}, \mathrm{D})$ mit 10 Äquivalenten $\mathrm{Nal}$ und einer Stoßenergie von $0 \mathrm{~V}(\mathrm{~A}, \mathrm{C}), 50 \mathrm{~V}$ $(B)$ und $70 \vee(D)$. In den markierten Bereichen treten einfach, zweifach und dreifach geladene Spezies $\left(\mathrm{P}^{+}, \mathrm{P}^{2+}\right.$ und $\left.\mathrm{P}^{3+}\right)$ auf.

Einfluss der Stoßenergie auf Polymerketten mit ionischer Endgruppe

Bei den PMA Proben mit ionischer Endgruppe wurde ebenfalls der Einfluss der Stoßenergie untersucht. Da im Massenspektrum von RAFT-PMA ${ }_{1 k^{-}} \mathrm{N}^{+}$keine bzw. kaum zweifach geladene Ketten entstehen, wurden nur RAFT-PMA ${ }_{3 k}-\mathrm{N}^{+}$und RAFT-PMA $7 \mathrm{k}^{-} \mathrm{N}^{+}$untersucht.

Die Abbildungen 4.18 A-D zeigen die ESI-Massenspektren von RAFT$\mathrm{PMA}_{3 \mathrm{k}}-\mathrm{N}^{+}$mit einer Stoßenergie von 0 bis $100 \mathrm{~V}$. Ohne die Zuführung von Stoßenergie treten einfach bis dreifach geladene Ketten auf (siehe auch Abschnitt 4.4.1. Bei den einfach geladenen Ketten im niedrigen $m / z$-Bereich handelt es sich entweder um Fragmente längerer Ketten oder um unverhältnismäßig häufig auftretende Ketten aufgrund von Massendiskriminierungseffekte. Die Erhöhung der Stoßenergie auf $50 \mathrm{~V}$ führt dazu, dass teilweise ein an der Polymerkette angelagertes Natriumion durch Stöße entfernt wird. Es treten nur noch einfach und zweifach geladene Spezies auf, wobei der Anteil der einfach geladenen Spezies gegenüber der zweifach geladenen zunimmt. Ab $70 \mathrm{~V}$ tritt die einfach geladene HR Spezies mit geringer Intensität auch oberhalb von $2500 \mathrm{~m} / \mathrm{z}$ auf. Bei einer Stoßenergie von $100 \mathrm{~V}$ ist die einfach geladene ZR Spezies dominierend und der Anteil an Fragmenten der HR Spezies ist immer 

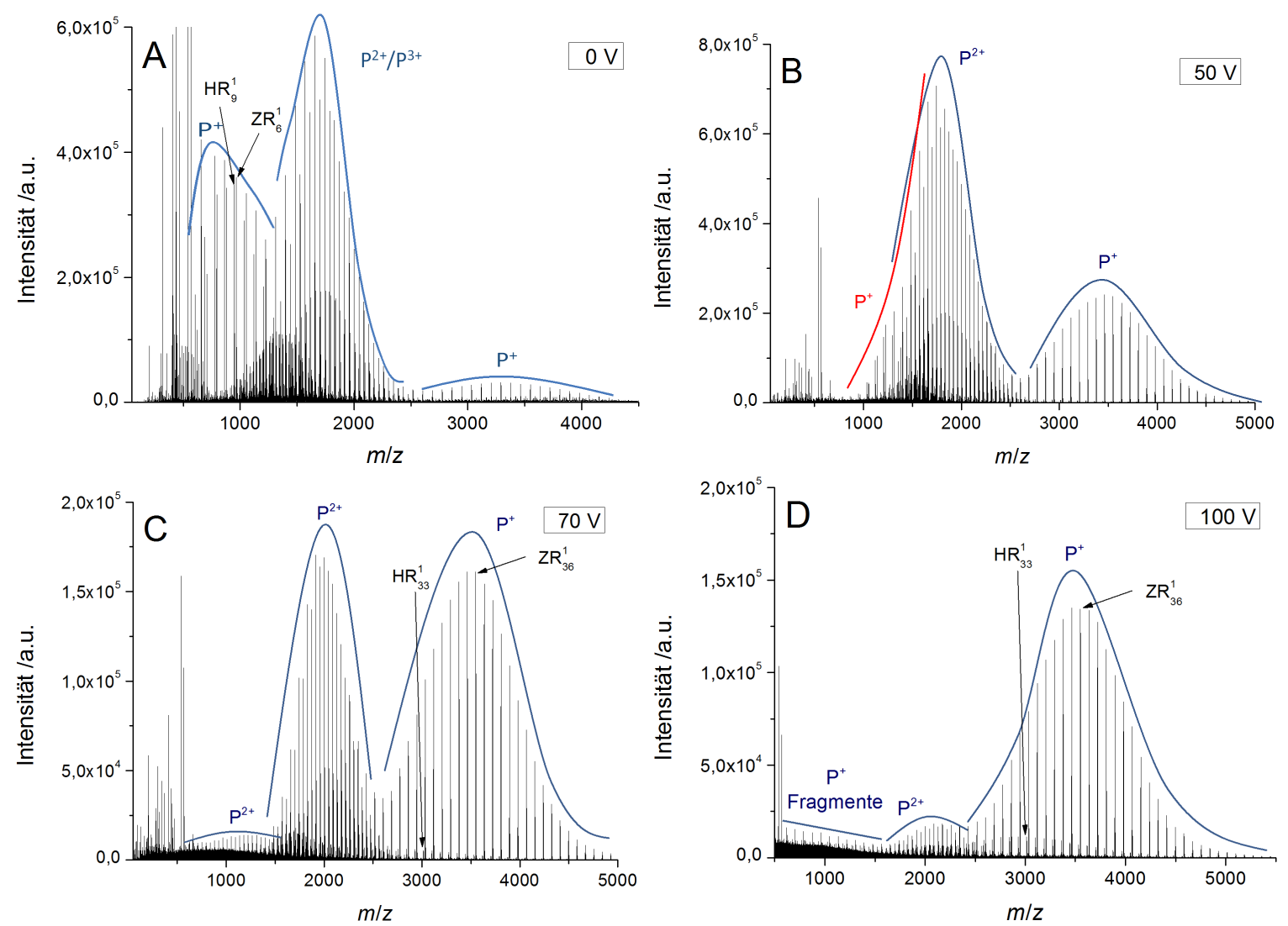

Abb. 4.18. Das ESI-Massenspektrum einer $50 \mu \mathrm{M}$ Lösung von RAFT-PMA $A_{3 k}-\mathrm{N}^{+}$gemessen in Aceton mit einer Stoßenergie von $0 \mathrm{~V}(\mathrm{~A}), 50 \mathrm{~V}(\mathrm{~B}), 70 \mathrm{~V}(\mathrm{C})$ und $100 \mathrm{~V}(\mathrm{D})$.

noch gering. Auch die Intensität der zweifach geladenen Ketten und einfach geladenen Fragmenten ist gering, sodass die Interpretation des Spektrums durch die Entfernung von Natriumionen mittels der Stoßenergie stark vereinfacht wird und die Molmassenverteilung der ZR Spezies leicht ausgewertet werden kann.

In den Abbildung 4.19 A bis $\mathrm{C}$ sind die Massenspektren der Probe RAFT-PMA $7 \mathrm{k}^{-} \mathrm{N}^{+}$mit einer Stoßenergie von 0, 50 und $120 \mathrm{~V}$ gezeigt. Zwischen einer Spannung von 0 und $50 \mathrm{~V}$ ist kaum ein Unterschied im Massenspektrum zu beobachten. In beiden Fällen werden einbis fünffach geladene Ketten detektiert. Bei $120 \mathrm{~V}$ werden an den Polymerketten angelagerte Natriumionen teilweise entfernt, wodurch nur noch ein- bis dreifach geladene Ketten auftreten, wobei die zweifach geladenen Spezies die häufigsten sind. Auch wenn die Anzahl von Natriumionen nicht vollständig auf eine je Polymerkette reduziert werden kann, wird durch die Stoßenergie die Komplexität des Spektrum erniedrigt. Dadurch können anhand der zweifach geladenen Ketten auch leicht die Molmassenverteilung und die Endgruppen analysiert werden. Abbildung 4.19 D zeigt einen Ausschnitt des Massenspektrums von RAFT-PMA ${ }_{7 k}-\mathrm{N}^{+}$mit einer höheren Konzentration in Aceton. Es werden bis $8000 \mathrm{~m} / \mathrm{z}$ einfach geladene HR und ZR Spezies erhalten, deren Isotopenmuster vom verwendeten ESI-TOF Synapt Massenspektrometer noch aufgelöst werden. Da das Massenspektrometer nur bis 

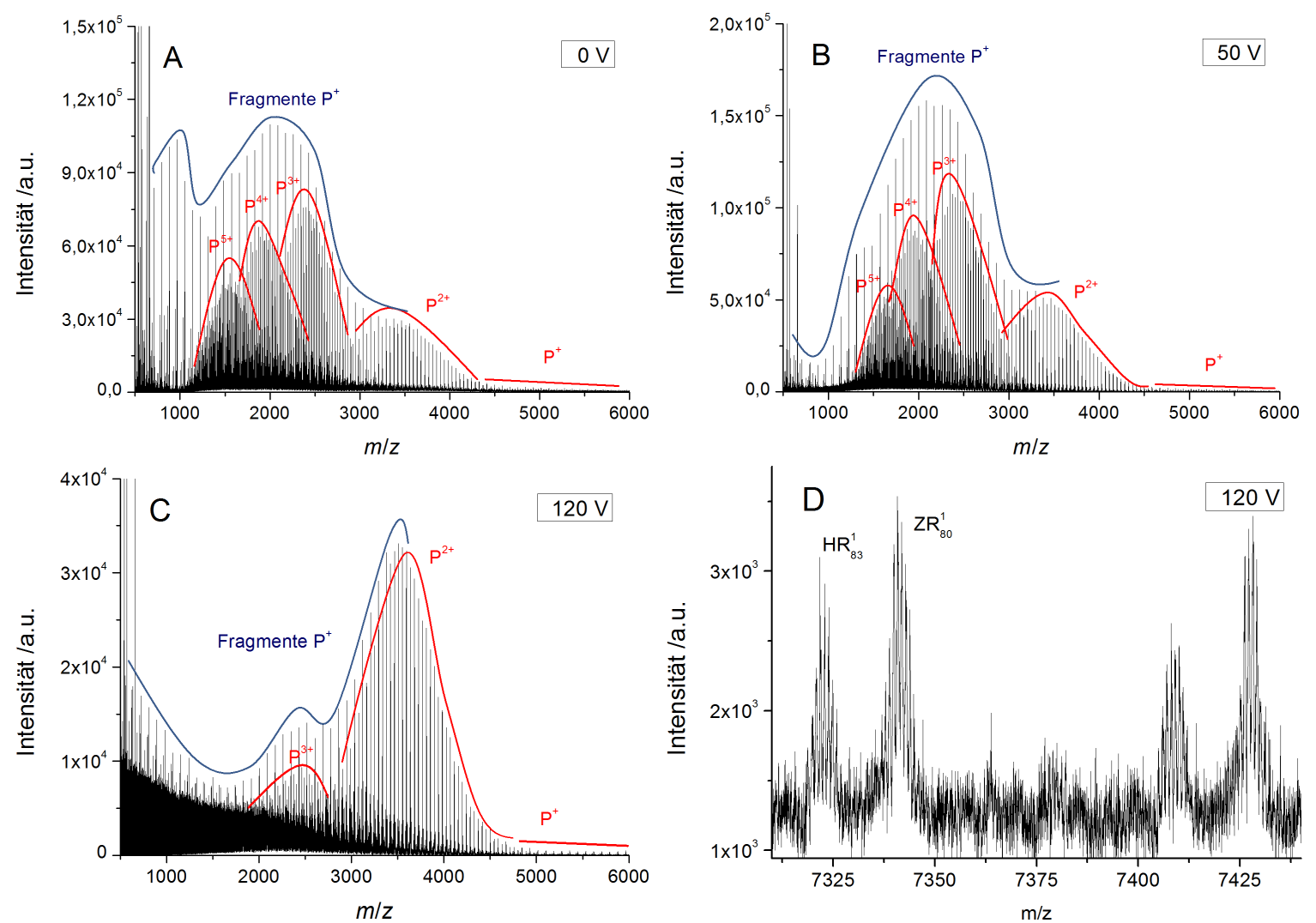

Abb. 4.19. Das ESI-Massenspektrum einer $50 \mu \mathrm{M}(\mathrm{A}-\mathrm{C})$ und $300 \mu \mathrm{M}$ (D) Lösung von RAFT$\mathrm{PMA}_{7 \mathrm{k}}-\mathrm{N}^{+}$gemessen in Aceton mit einer Stoßenergie von $0 \mathrm{~V}(\mathrm{~A}), 50 \mathrm{~V}(\mathrm{~B})$ und $120 \mathrm{~V}(\mathrm{C}, \mathrm{D})$.

$2000 \mathrm{~m} / \mathrm{z}$ kalibriert wurde, steigt die Differenz zwischen den experimentell ermittelten und den berechneten $\mathrm{m} / \mathrm{z}$-Werten mit steigendem $\mathrm{m} / \mathrm{z}$ an (siehe Tabelle B10 im Anhang).

Zusammenfassend können durch die zugefügte Stoßenergie die Anzahl von Natriumionen je Polymerkette reduziert und die Polymerketten entclustert werden. Dabei ist die Reduktion von Natriumionen effektiver bei Polymeren mit ionischer Endgruppe. Bei Polymeren ohne ionische Endgruppe entstehen durch die Zufügung von Stoßenergie zudem unbekannte Fragmente. 


\subsection{MALDI-MS Analyse der RAFT-Polymere}

Die RAFT-Polymere wurden auch mittels MALDI-MS untersucht. Dabei lag der Fokus auf dem Vergleich der Spektrenqualität der Polymere mit und ohne ionische Endgruppe. Die Polymere wurden in Aceton oder THF gelöst und mit der Lösung der Matrix und gegebenenfalls des Salzes zur lonisierung (bei Polymeren ohne ionische Endgruppe) in THF gemischt. Bei Polymeren mit tertiärer Aminendgruppe wurde eine geringe Menge Essigsäure der Mischung aus Polymer- und Matrixlösung direkt zugesetzt. Als Matrix wurde in allen Fällen DCTB verwendet, da sie die besten Spektren lieferte. Die Lösungen wurden anschließend auf die Probenplatte gespottet. Zur Analyse der Probenspots wurde der Reflektronmodus verwendet, um eine möglichst hohe Massenauflösung zu erhalten. Bei der Spektrenaufnahme wurde generell festgestellt, dass die Reproduzierbarkeit in der MALDI-MS wesentlich geringer war als in der ESI-MS. Die Spektren unterscheiden sich von Aufnahme zu Aufnahme nicht nur in den Intensitäten und dem S/R-Verhältnis, sondern auch in der Molmassenverteilung und in dem Peakmaximum. Für die Benennung der verschiedenen Spezies wird die bereits aus Abschnitt 4.4 bekannte Nomenklatur verwendet.

\subsubsection{Analyse der Polymethylacrylate}

\section{PMA ohne ionische Endgruppe}

Abbildungen 4.20 A bis $D$ zeigen die MALDI-Massenspektren von RAFT-PMA $2 k^{-}$ $\mathrm{COOH}$ und RAFT-PMA $4 \mathrm{k}-\mathrm{COOH}$ aufgenommen mit einer optimierten (links) und höheren (rechts) Laserenergie. In allen Fällen werden die einfach geladene reversibel deaktivierte Spezies ${ }^{N a} Z^{1}{ }^{1}$ und die analoge Spezies ${ }^{2 N a}{ }^{2} R^{1}$, bei der ein Proton der terminalen Carboxylendgruppe durch ein Natriumion ersetzt ist, detektiert. Eine Mehrfachladung der Polymere tritt in der MALDI-MS erst bei sehr hohen Molmassen (meistens erst über $100000 \mathrm{~g} \mathrm{~mol}^{-1}$ ) auf. $68 \mathrm{~lm}$ niedrigeren $\mathrm{m} / \mathrm{z}$ Bereich von RAFT-PMA $A_{2 k}-\mathrm{COOH}$ treten zudem unbekannte Fragmente mit $\Delta=-5$ bis -6 $\mathrm{m} / \mathrm{z}$ zu den berechneten Werten der beiden zuvor genannten Spezies auf. Aufgrund der geringen S/R-Verhältnisse (Tabelle 4.11) können ebenfalls zu erwartende Spezies wie z.B. ZI nicht nachgewiesen werden. Im Massenspektrum von RAFT-PMA ${ }_{2 k}-\mathrm{COOH}$ mit optimierter Laserenergie (Abb. 4.20 A) zeigt das Innenbild, dass das Isotopenmuster zumindest teilweise aufgelöst werden kann. Bei Verwendung einer höheren Laserenergie (Abb. 4.20 B) geht die Isotopenauflösung aufgrund der verbreiterten kinetischen Engergieverteilung der lonen verloren, was dann trotz verzögerter lonenextraktion zu einer Verbreiterung der Peaks und einer Abnahme der Massenauflösung führt. ${ }^{3970179}$ Bei RAFT-PMA 4 k-COOH kann das Isotopenmuster weder bei Verwendung einer niedrigen noch bei einer höheren Laserenergie aufgelöst werden. Bei beiden Polymeren führt die Verwendung einer höheren Laserenergie zu intensiveren Signalen, jedoch steigt auch dann das Rauschen an. Daher nimmt das S/R-Verhältnis mit zunehmender Laserenergie nicht zu. 

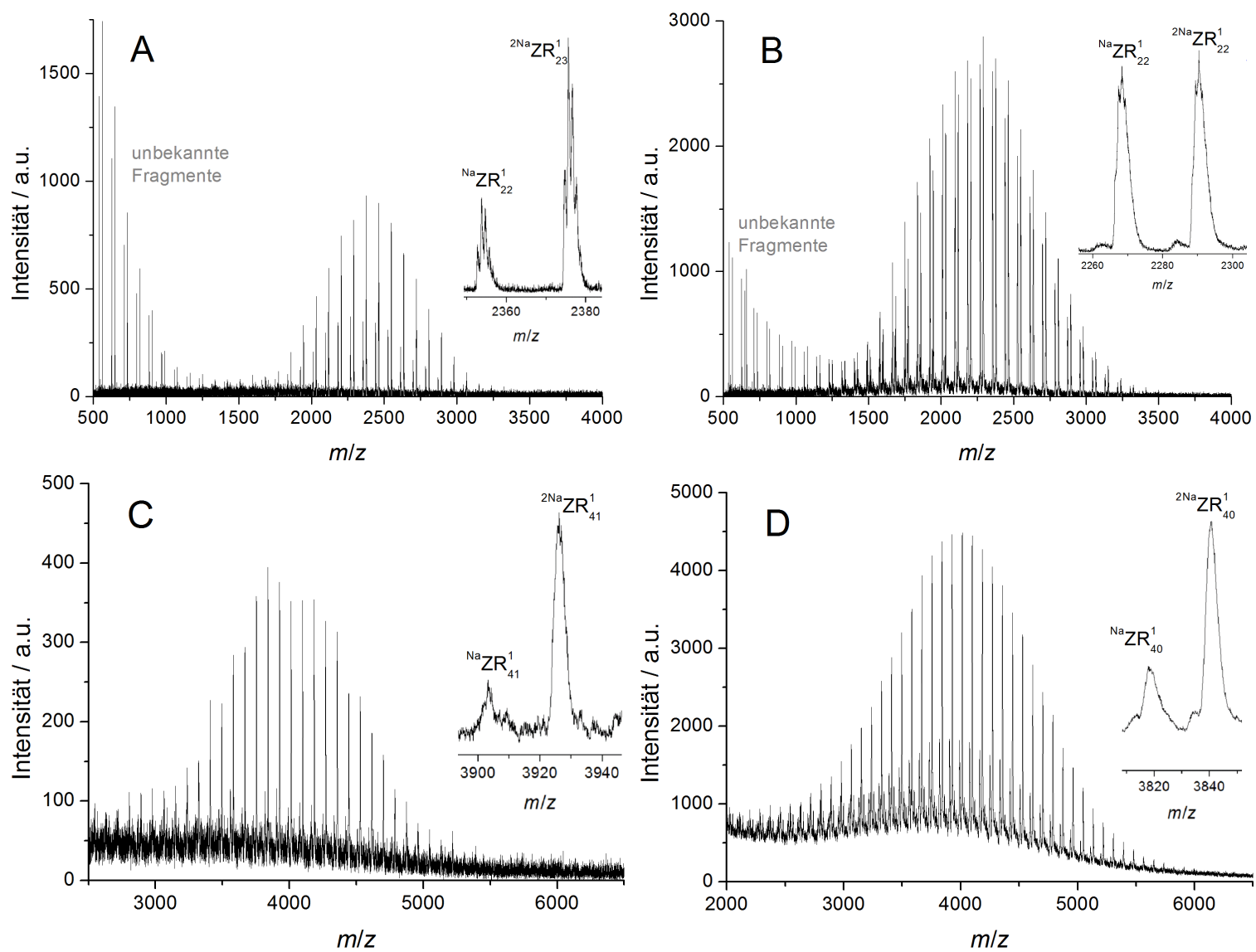

Abb. 4.20. Das MALDI-Massenspektrum von RAFT-PMA $A_{2 k}-C O O H(A, B)$ und RAFT-PMA $A_{4 k}-$ $\mathrm{COOH}(C, D)$ im Reflektronmodus. Die Spektren wurden mit optimierter Laserenergie $(A, C)$ und etwas höherer Laserenergie (B, D) aufgenommen. Die Innenbilder zeigen einen vergrößerten Ausschnitt des Spektrums.

Tab. 4.11. S/R-Verhältnisse, Auflösung des Isotopenmusters und die absoluten Signalintensitäten in Abhängigkeit von der Laserenergie am Peakmaximum $\left(M_{\mathrm{p}}\right)$ der mit dem RAFT-Agens RAFT-COOH hergestellten PMA.

\begin{tabular}{|c|c|c|c|c|}
\hline Polymer & Laserenergie & S/R-Verhältnis $\left(M_{\mathrm{p}}\right)$ & Intensität $\left(M_{\mathrm{p}}\right)$ / a.u. & Isotopenmuster \\
\hline $\mathrm{PMA}_{2 \mathrm{k}}$ & niedrig $^{a}$ & 33 & 930 & teilaufgelöst \\
\hline $\mathrm{PMA}_{2 \mathrm{k}}$ & höher気 & 32 & 2900 & nicht aufgelöst \\
\hline $\mathrm{PMA}_{4 \mathrm{k}}$ & 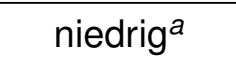 & 8 & 390 & nicht aufgelöst \\
\hline $\mathrm{PMA}_{4 \mathrm{k}}$ & höher国 & $5-6$ & 4500 & nicht aufgelöst \\
\hline $\mathrm{PMA}_{7 \mathrm{k}}$ & niedrig $^{b}$ & 13 & 4100 & nicht aufgelöst \\
\hline $\mathrm{PMA}_{7 \mathrm{k}}$ & höher & 5 & 500 & nicht aufgelöst \\
\hline
\end{tabular}

${ }^{a}$ Im Reflektronmodus gemessen.

${ }^{b} \mathrm{Im}$ linearen Modus gemessen. 
Das Spektrum RAFT-PMA ${ }_{7 \mathrm{k}}-\mathrm{COOH}$ in Abbildung 4.21A weist ebenfalls ein schlechtes S/R-Verhältnis auf. Mit steigender Molmasse wird es schwieriger, eine befriedigende Peakintensitäten zu erhalten, da Massendiskriminierungseffekte (siehe Abschnitt 2.3.3) eine gröBere Rolle spielen und höher molekulare Proben schlechter desorbieren. ${ }^{707185}$ Der verwendete Reflektronmodus reduziert zusätzlich das Signal der lonen. Für Polymere mit Molmassen oberhalb von $6000 \mathrm{~g} \mathrm{~mol}^{-1}$ wird mit dem verwendeten MALDI-Massenspektromter das Isotopenmuster auch unter optimierten Bedingungen nicht mehr aufgelöst. Daher kann für die Verbesserung der Sensitivität auch der lineare Modus verwendet werden. Das Massenspektrum in Abbildung 4.21 $\mathrm{C}$ wurde von RAFT-PMA ${ }_{7 \mathrm{k}}-\mathrm{COOH}$ im linearen Modus aufgenommen. Obwohl die Laserenergie geringer ist (35\% statt $60 \%$ im Reflektronmodus) sind die Peakintensitäten und das S/R-Verhältnis höher. Im Folgenden wird aber auch bei Polymeren mit höheren Molmassen ausschließlich der Reflektronmodus verwendet, um eine bessere Vergleichbarkeit der Messungen zu gewährleisten.

Tabelle B11 im Anhang zeigt die berechneten und gefundenen $m / z$-Werte sowie deren

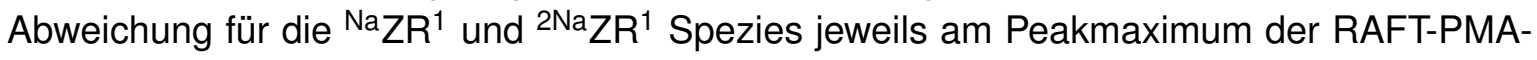
$\mathrm{COOH}$-Polymere. Die Massengenauigkeit ist geringer als im ESI-Massenspektrometer, selbst wenn die Kalibrierung direkt vor der Messung der Probe stattgefunden hat. Mit zunehmender Molmasse steigen die Abweichungen zwischen theoretischen und experimentellen Massen weiter an, da die externe Kalibrierung des MALDI-Massenspektrometers nur bis $2000 \mathrm{~m} / \mathrm{z}$ durchgeführt wurde.

Die MALDI-Massenspektren der Polymere mit protonierter tertiärer Aminendgruppe sind in den Abbildungen 4.22 A bis $\mathrm{C}$ dargestellt. Bei RAFT-PMA ${ }_{1 \mathrm{k}}$-Amin wird die Spezies ${ }^{\mathrm{H}} \mathrm{ZR}^{1}$ mit protonierter Endgruppe detektiert. Analog zu RAFT-PMA ${ }_{2 k}-\mathrm{COOH}$ entstehen im niedrigen $m / z$-Bereich ebenfalls unbekannte Fragmente mit $\Delta=-5$ bis $-6 \mathrm{~m} / \mathrm{z}_{\mathrm{zur}} \mathrm{H}_{\mathrm{ZR}}{ }^{1}$ Spezies. Bei RAFT-PMA ${ }_{3 k}-$ Amin und RAFT-PMA ${ }_{7 k}-$ Amin werden die Ketten entweder über Protonierung oder auch durch Natriumionen ionisiert (Abb. 4.22 B und $C$ ), obwohl kein Natriumsalz zugegeben wurde. Mit zunehmender Kettenlänge wird eine lonisierung mittels Natrium
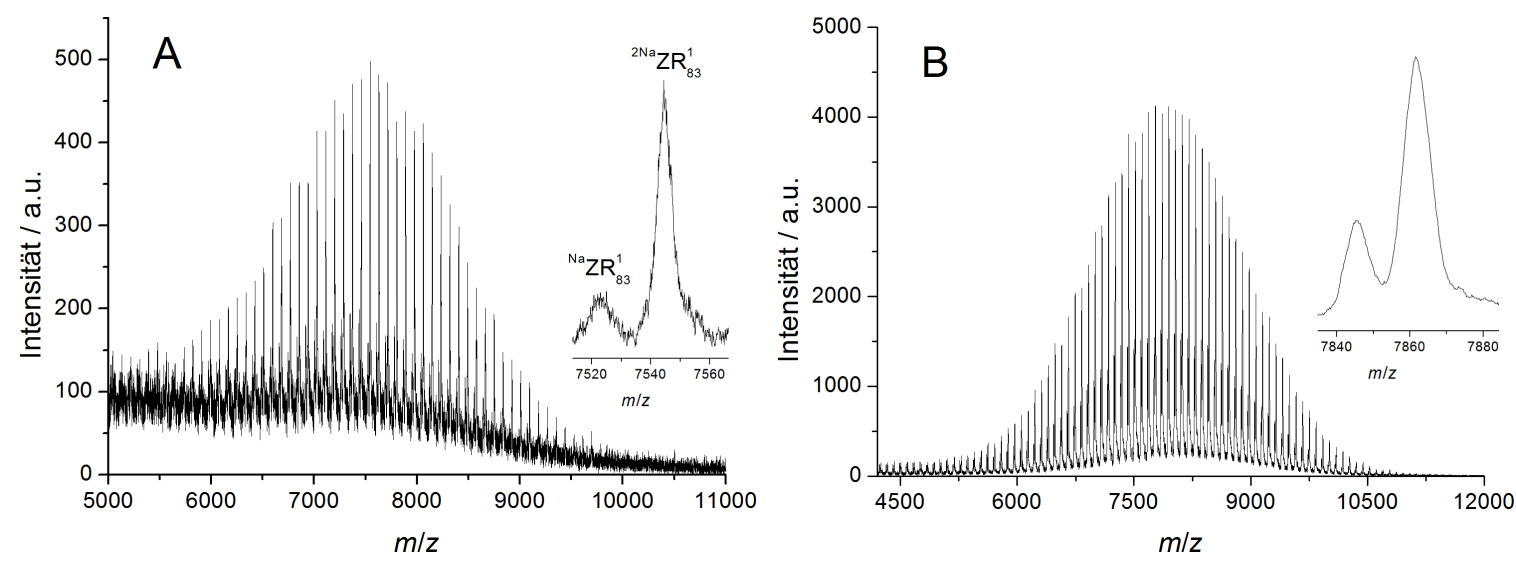

Abb. 4.21. Das MALDI-Massenspektrum von RAFT-PMA $A_{7 k}-\mathrm{COOH}$ im Reflektronmodus $(A)$ und im linearen Modus (B) mit DCTB als Matrix. Die Innenbilder zeigen einen vergrößerten Ausschnitt des Spektrums. 
wahrscheinlicher, da mehr Bindungsstellen für Natrium vorhanden sind, wohingegen es weiterhin nur eine basische Endgruppe gibt.

Analog zu RAFT-PMA-COOH sinkt mit zunehmender Kettenlänge der Polymere das S/RVerhältnis (Tabelle 4.12). Die Probe RAFT-PMA ${ }_{7 k}-$ Amin wurde im linearen Modus gemessen, um ein ausreichendes Signal zu erhalten. Im Vergleich zu RAFT-PMA ${ }_{3 k}-A m i n$ steigt daher das S/R-Verhältnis wieder an. Eine protonierte basische Endgruppe in PMA liefert gegenüber Natriumaddukten, wie auch bereits in der ESI-MS, keinen Vorteil in der Sensitivität oder im S/R-Verhältnis. Tabelle B12 zeigt die berechneten und gefundenen $\mathrm{m} / \mathrm{z}$-Werte sowie deren Abweichung für die ${ }^{H} Z^{1}{ }^{1}$ und ${ }^{N a} Z^{1}$ Spezies jeweils am Peakmaximum der RAFT-PMA-Amin-Polymere.
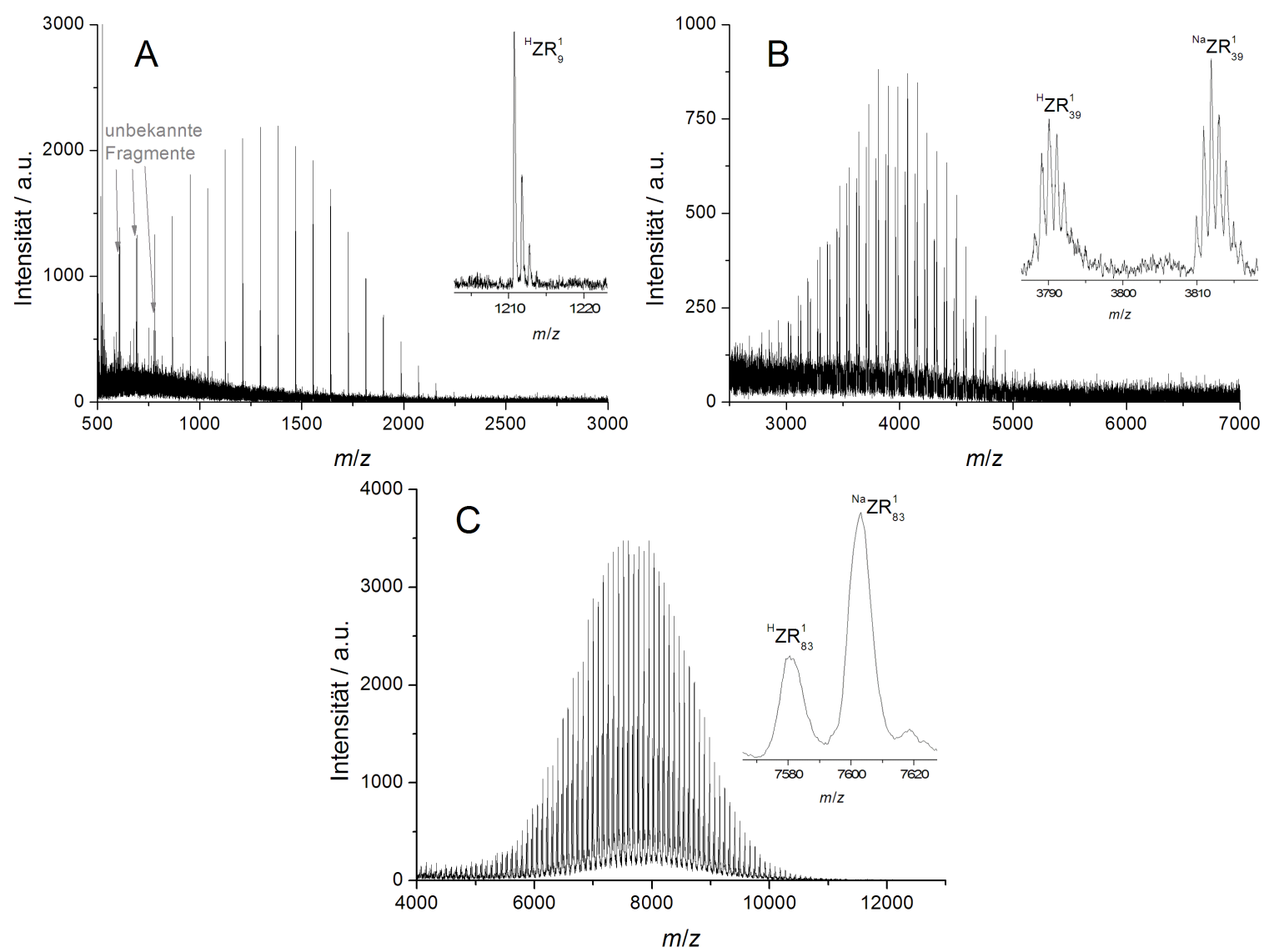

Abb. 4.22. Das MALDI-Massenspektrum von RAFT-PMA ${ }_{1 k}-A m i n(A ; i m$ Reflektronmodus), RAFT-PMA ${ }_{3 k}$-Amin (B; im Reflektronmodus) und RAFT-PMA $A_{7 k}$-Amin ( $C$; im linearen Modus) mit DCTB als Matrix. Die Innenbilder zeigen, ob eine Isotopenauflösung vorhanden ist oder nicht. 
Tab. 4.12. S/R-Verhältnisse, die absoluten Signalintensitäten und Auflösung des Isotopenmusters am Peakmaximum $\left(M_{\mathrm{p}}\right)$ der mit dem RAFT-Agens RAFT-Amin hergestellten PMA.

\begin{tabular}{lccc}
\hline Polymer & S/R-Verhältnis $\left(M_{\mathrm{p}}\right)$ & Intensität $\left(M_{\mathrm{p}}\right) /$ a.u. & Isotopenmuster \\
\hline $\mathrm{PMA}_{1 \mathrm{k}}$ & 22 & 2200 & aufgelöst \\
$\mathrm{PMA}_{3 \mathrm{k}}$ & 9 & 880 & teilaufgelöst \\
$\mathrm{PMA}_{7 \mathrm{k}}{ }^{\natural}$ & 17 & 3500 & nicht aufgelöst \\
\hline
\end{tabular}

${ }^{a}$ Gemessen im linearen Modus.

\section{PMA mit ionischer Endgruppe}

Die Abbildungen $4.23 \mathrm{~A}, \mathrm{C}$ und $\mathrm{E}$ zeigen die MALDI-Massenspektren von RAFT-PMA ${ }_{1 \mathrm{k}^{-}}$

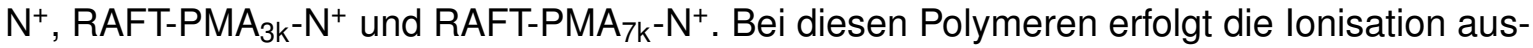
schließlich über die geladene Endgruppe und es werden keine Addukte gebildet. Hauptsächlich werden die reversibel deaktivierte Polymerketten der Spezies ZR ${ }^{1}$ detektiert. Zu einem geringen Anteil treten auch Ketten der Spezies $H R^{1}$ auf, bei denen die Z-Gruppe inklusive der Trithiocarbonatgruppe durch ein $\mathrm{H}$-Atom ersetzt worden ist. Die HR ${ }^{1}$ Spezies wurde bei den Polymeren ohne ionische Endgruppe in der MALDI-MS nicht gefunden, da bei diesen Polymeren das S/R-Verhältnis wesentlich kleiner ist. Möglich wäre, dass diese Abspaltung photochemisch stattfindet. Die Trithiocarbonatgruppe absorbiert Licht der Wellenlänge des verwendeten Lasers (355 nm) und die photoinduzierte homolytische Spaltung der C-S-Bindung zwischen der Polymerkette und der Trithiocarbonatgruppe ist literaturbekannt. 193/194 Das polymere Radikal kann dann beispielsweise von einem Matrixmolekül oder einem anderen $\mathrm{H}$-Donor ein Wasserstoffatom abstrahieren, wodurch dann die HR Spezies entsteht. Die Signale der HR Spezies sind gegenüber der ZR Spezies jedoch stark zu geringeren $\mathrm{m} / \mathrm{z}$-Werten verschoben, sodass auch eine Fragmentierung innerhalb der Repetiereinheiten der Polymerkette eine mögliche Erklärung für diese Spezies sein könnte. Tabelle B13 im Anhang zeigt die berechneten und experimentell ermittelten $\mathrm{m} / \mathrm{z}$-Werte sowie deren Abweichung für die $\mathrm{ZR}^{1}$ und $\mathrm{HR}^{1}$ Spezies jeweils am Peakmaximum der RAFT-PMA$\mathrm{N}^{+}$-Polymere.

Als nächstes werden die Ergebnisse der MALDI-MS von den RAFT-PMA-COOH- und RAFT-PMA-N ${ }^{+}$-Polymeren miteinander verglichen. Die Spektrenqualität der RAFT-PMAAmin-Polymere ist ähnlich zu denen der RAFT-PMA-COOH-Polymere und daher werden deren Spektren hier nicht nochmal dargestellt. In den Abbildungen 4.23 A bis E sind die Massenspektren der RAFT-PMA-COOH- (rechts) und RAFT-PMA-N+-Polymere (links) mit ähnlicher Molmasse nebeneinander dargestellt. Die PMA mit ionischer Endgruppe weisen alle ein wesentlich niedrigeres Rauschniveau und höhere Signalintensitäten auf als die jeweiligen Pendants der durch Natriumadduktbildung ionisierten RAFT-PMA-COOH-Polymere. Dadurch sind die S/R-Verhältnisse, wie anhand der Tabellen 4.11 und 4.13 ersichtlich ist, bei den drei RAFT-PMA-N+-Polymeren um den Faktor 40, 75 und 140 höher als bei den 
RAFT-PMA-COOH-Polymeren. Vor allem bei den Polymeren mit höherer Molmasse konnte somit die Sensitivität maßgeblich verbessert werden. Dies ist in der Spurenanalyse oder in der Analyse von Spezies, die nur zu einem geringen Anteil vorhanden sind, von großem Vorteil.
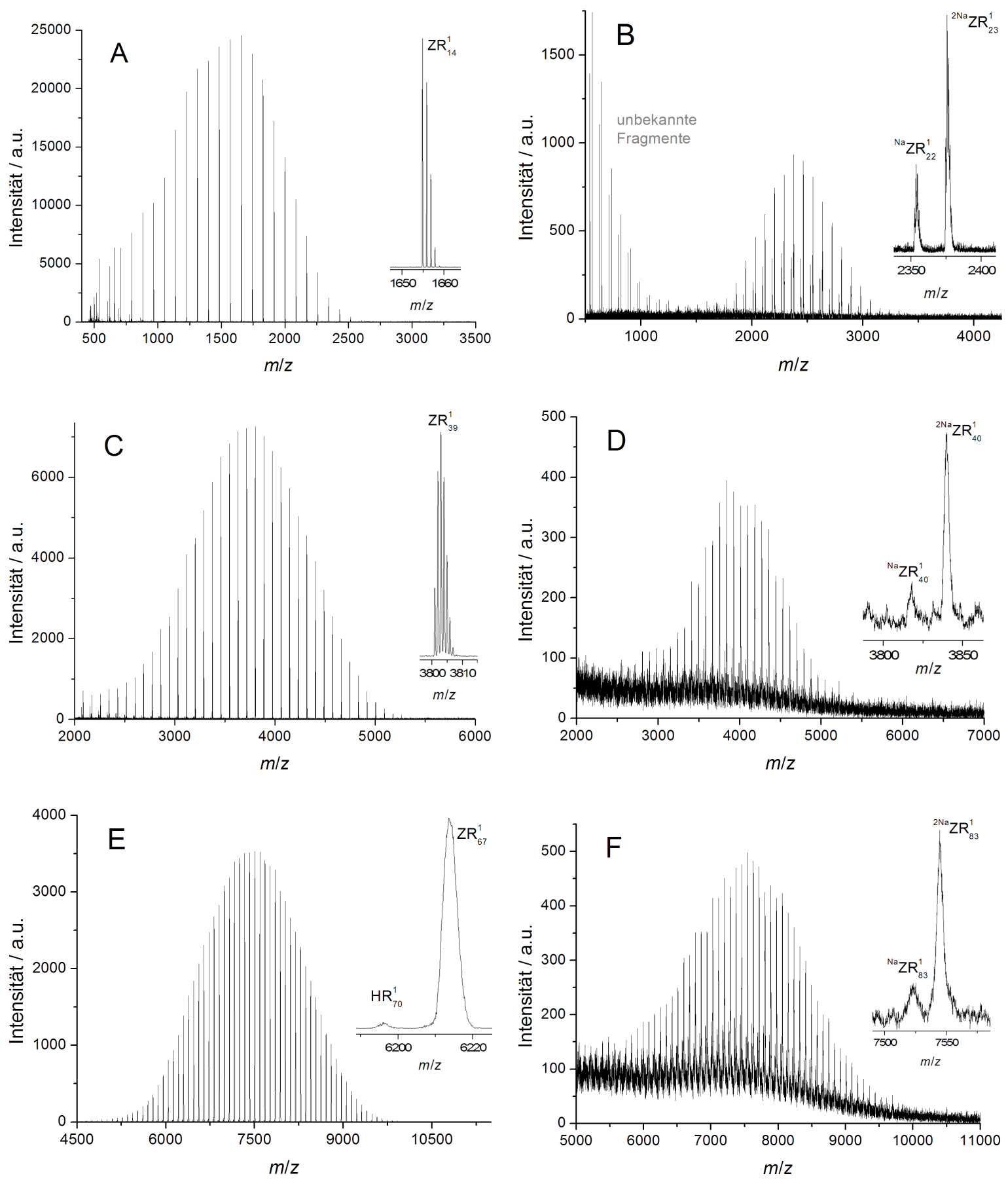

Abb. 4.23. Die MALDI-Massenspektren der Polymere RAFT-PMA $A_{1 k}, 3 k, 7 k^{-} \mathrm{N}^{+}(A, C, E)$ und RAFT-

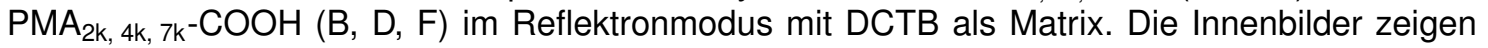
jeweils einen vergrößerten Ausschnitt des Spektrums. 
Tab. 4.13. S/R-Verhältnisse, die absoluten Signalintensitäten und Auflösung des Isotopenmusters am Peakmaximum $\left(M_{\mathrm{p}}\right)$ der mit dem RAFT-Agens RAFT-N ${ }^{+}$hergestellten PMA wurden.

\begin{tabular}{cccc}
\hline Polymer & S/R-Verhältnis $\left(M_{\mathrm{p}}\right)$ & Intensität $\left(M_{\mathrm{p}}\right) /$ a.u. & Isotopenmuster \\
\hline $\mathrm{PMA}_{1 \mathrm{k}}$ & 1300 & 25000 & aufgelöst \\
$\mathrm{PMA}_{3 \mathrm{k}}$ & 600 & 7300 & aufgelöst \\
$\mathrm{PMA}_{7 \mathrm{k}}$ & 700 & 3500 & nicht aufgelöst \\
\hline
\end{tabular}

Ein weiterer Punkt zur Beurteilung der Spektrenqualität ist die erhaltene Massenauflösung $R$. Die in den Spektren erhaltenen Massenauflösungen der RAFT-PMA-COOH, RAFT-PMAAmin- und RAFT-PMA-N ${ }^{+}$-Polymere sind in Tabelle 4.14 gezeigt. Die Massenauflösung wurde dabei anhand der Halbwertsbreite des Peaks am Maximum (englisch full width at half maximum, FWHM) bestimmt (Gleichung 4.2, wie es für TOF-Analysatoren üblich ist. ${ }^{\text {[39] }}$

$$
R=\frac{m}{\Delta m}=\frac{m}{\mathrm{FWHM}}
$$

$R$ ist bei den RAFT-PMA-N ${ }^{+}$-Polymeren mit niedriger und mittlerer Molmasse um den Faktor 1,5 bis 10 höher als bei den den PMA mit terminaler Amin bzw. Carboxylgruppe. Die ist auch qualitativ in den Innenbildern der Abbildungen 4.23 A bis $\mathrm{F}$ zu erkennen. So sind in Abb. 4.23 $A$ die Isotopenpeaks wesentlich schmaler als in Abb. 4.23 $B$ und bei RAFT-PMA $3 k^{-} \mathrm{N}^{+}$sind die Isotopenpeaks vollständig voneinander getrennt, wohingegen sie bei RAFT-PMA $3 \mathrm{k}-\mathrm{Amin}_{\mathrm{n}}$ (Abb. 4.22 B) nur teilweise voneinander getrennt und bei RAFT-PMA $\mathrm{k}_{\mathrm{k}}-\mathrm{COOH}$ (Abb. $4.23 \mathrm{~B}$ ) gar nicht voneinander getrennt sind. Wie bereits in Abschnitt 2.3.2 erwähnt, ist $R$ in der MALDI-MS im Gegensatz zur ESI-MS stark abhängig von der Homogenität der Probe und der Laserenergie. ${ }^{39179180}$ Bei den RAFT-PMA-N+2-Polymeren werden niedrigere Laserenergien benötigt, um intensivere Signale zu erhalten. Daraus resultiert dann auch die höhere Massenauflösung. Bei den drei Polymeren mit hoher Molmasse (ca. $7 \mathrm{~kg} \mathrm{~mol}^{-1}$ ) kann auch bei dem Polymer mit ionischer Endgruppe das Isotopenmuster nicht mehr aufgelöst werden. Die Massenauflösungen von RAFT-PMA $7 \mathrm{k}^{-\mathrm{N}^{+}}$und RAFT-PMA $\mathrm{Ak}_{\mathrm{k}}-$ Amin betragen in etwa 1400 und die von RAFT-PMA $A_{7 k}-\mathrm{COOH}$ ist mit 900 etwas geringer, da letztere Probe im linearen Modus gemessen wurde.

Tab. 4.14. Die erhaltenen Massenauflösungen $R$ am Peakmaximum für verschiedene Polymere im Reflektronmodus. Die Massenauflösung wurde anhand der Gleichung 4.2 bestimmt.

\begin{tabular}{cc|cc|cc}
\hline Polymer & $R$ & Polymer & $R$ & Polymer & $R$ \\
\hline RAFT-PMA $_{1 \mathrm{k}}-\mathrm{N}^{+}$ & 19000 & RAFT-PMA $_{2 \mathrm{k}}-\mathrm{COOH}$ & 3600 & RAFT-PMA $_{1 \mathrm{k}}-$ Amin & 4800 \\
RAFT-PMA $_{3 \mathrm{k}}-\mathrm{N}^{+}$ & 12000 & RAFT-PMA $_{4 \mathrm{k}}-\mathrm{COOH}$ & 1200 & RAFT-PMA $_{3 \mathrm{k}}-\mathrm{Amin}_{1}$ & 8100 \\
RAFT-PMA $_{7 \mathrm{k}}-\mathrm{N}^{+}$ & 1400 & RAFT-PMA $_{7 \mathrm{k}}-\mathrm{COOH}$ & 1400 & PMA $_{7 \mathrm{k}}-$ Amin $^{\text {R }}$ & 900 \\
\hline
\end{tabular}

${ }^{a}$ Gemessen im linearen Modus. 
In der MALDI-MS werden in der Regel nur einfach geladene Ketten erhalten (siehe Abschnitt 2.3.2). Erst bei sehr hohen Molmassen werden zweifach geladene Ketten detektiert. 68 Die Probe RAFT-PMA ${ }_{2 k}-\mathrm{N}^{2+}$ wurde in der MALDI-MS analysiert, um festzustellen was in der MALDI-MS geschieht, wenn die Polymerkette bereits zwei permanente Ladungen besitzt. In Abbildung 4.24 ist das MALDI-Massenspektrum von RAFT-PMA $2 \mathrm{k}-\mathrm{N}^{2+}$ gezeigt. Obwohl die Kette durch zwei Ammoniumgruppen zweifach positiv geladen ist, werden nur einfach geladene Ketten erzeugt. Eine der zwei detektierten einfach geladenen Verteilungen kann der ' $\mathrm{ZR}^{+}$Spezies zugeordnet werden. In diesem Fall ist einer der beiden Ammoniumgruppen der Polymerkette durch ein lodidion neutralisiert. lodid ist neben Bromid eines der beiden Gegegenionen im verwendeten zweifach geladenen RAFT-Agens. Der zweiten einfach geladenen Spezies konnte keine Struktur zugeordnet werden.

Eine mögliche Erklärung für die Detektion von nur einfach geladenen Polymerketten könnte das in Abschnitt 2.3.2 erklärte lucky survivor Modell liefern. Im Falle von mehrfach geladenen Polymerketten ist die Rekombination von Kationen und Anionen in der Gasphase wesentlicher schneller. ${ }^{39169}$ Der Vorteil, den die einfach geladene Kette bei den RAFT-PMA$\mathrm{N}^{+}$-Polymere in Bezug auf die Peakintensitäten und das S/R-Verhältnis bringt, ist bei RAFT$\mathrm{PMA}_{2 \mathrm{k}}-\mathrm{N}^{2+}$ nicht mehr gegeben. Das S/R-Verhältnis in Abbildung 4.24 beträgt am Peakmaximum nur 13. Zudem war es in keinem Spektrum möglich das Isotopenmuster aufzulösen, obwohl die Molmasse des Polymers noch recht gering ist.

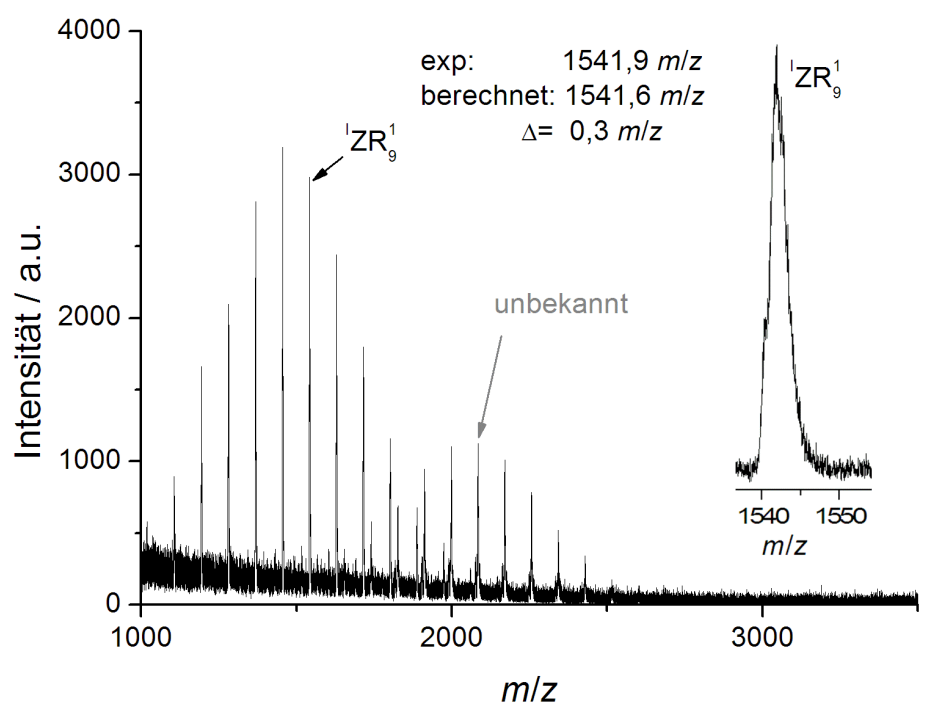

Abb. 4.24. Das MALDI-Massenspektrum von RAFT-PMA ${ }_{2 k}-\mathrm{N}^{2+}$ im Reflektronmodus mit DCTB als Matrix. Das Innenbild zeigt einen vergrößerten Ausschnitt des Spektrums. 


\subsubsection{Analyse der Polystyrole}

Die Abbildungen 4.25 A bis $C$ zeigen die MALDI-Massenspektren der Polymere RAFT-PS ${ }_{2 k^{-}}$

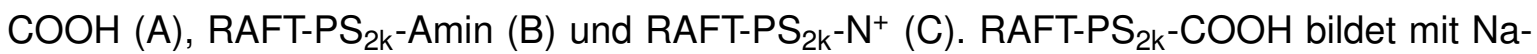
trium keine stabilen Addukte in der MALDI-MS, weshalb mit Zugabe von Nal kein Massenspektrum des Polymers aufgenommen werden konnte. Daher wurde Silbertrifluoressigsäure zugegeben, um die Ketten durch Komplexbildung mit Silberionen zu ionisieren. In der Literatur wurde gezeigt, dass Silber und andere Übergangsmetalle die Dithiobenzoatgruppe der RAFT-Polymere abspaltet und zu vinylterminierten Polymerketten führt. 174-176 Auch die Z-Gruppe inklusive der Trithiocarbonatgruppe wird bei RAFT-PS ${ }_{2 k}-\mathrm{COOH}$ durch Silber abgespalten. Diese Abspaltung und die Entstehung der vinylterminierten Polymerketten der Spezies UR kann anhand des in der Literatur vorgeschlagenen und in Schema 4.7 dargestellten Mechanismus erklärt werden. ${ }^{[174}$ Die Komplexierung des Silberions durch die Trithiocarbonatgruppe führt zur E1 Eliminierung. Die berechneten und experimentell bestimmten $\mathrm{m} / \mathrm{z}$-Werte finden sich im Anhang in Tabelle B14.
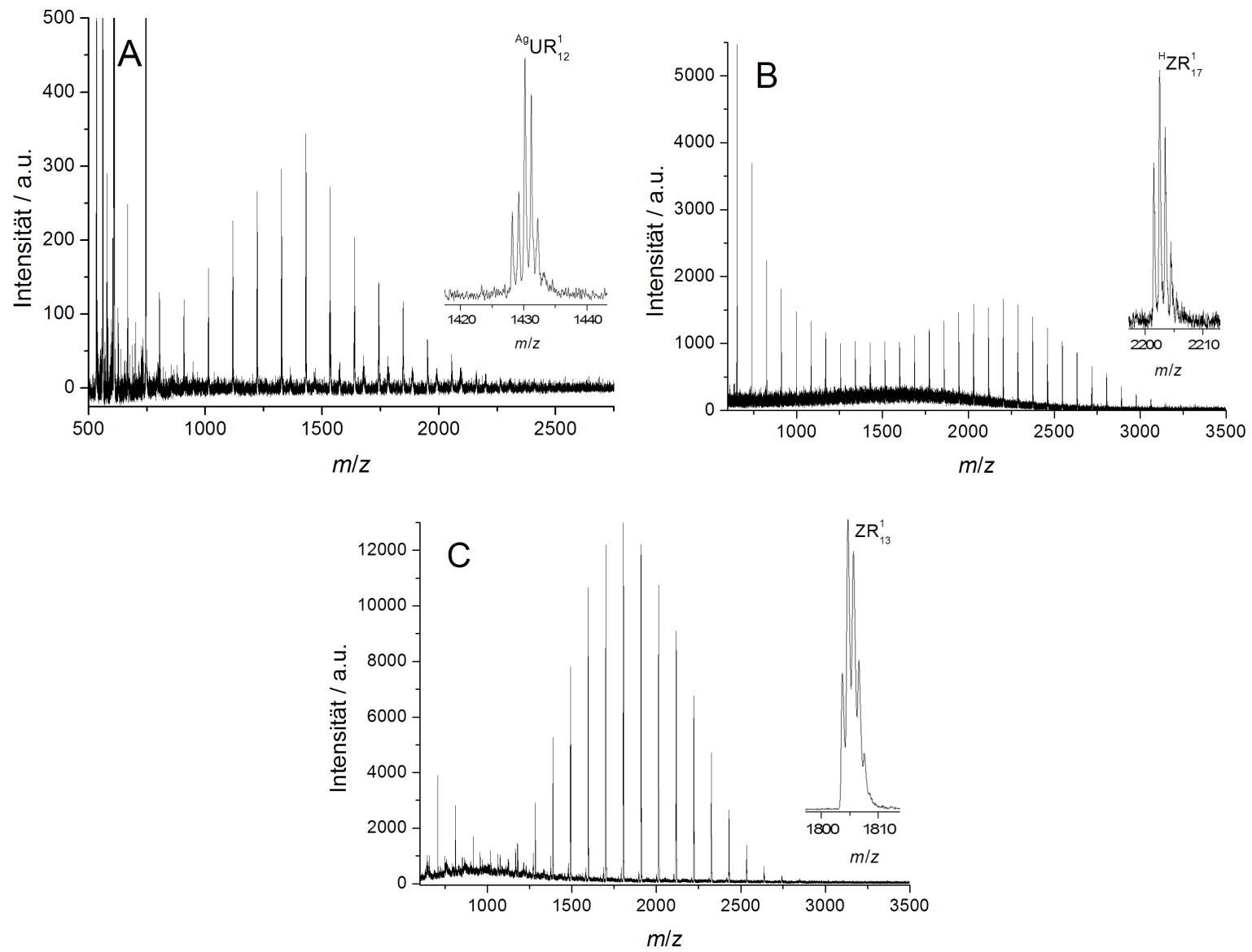

Abb. 4.25. Das MALDI-Massenspektrum von RAFT-PS ${ }_{2 k}-C O O H(A), R A F T-P S_{2 k}-A m i n(B)$ und RAFT-PS $2 k^{-} \mathrm{N}^{+}(C)$ im Reflektronmodus mit DCTB als Matrix. Die Innenbilder zeigen jeweils einen vergrößerten Ausschnitt des Spektrums. 


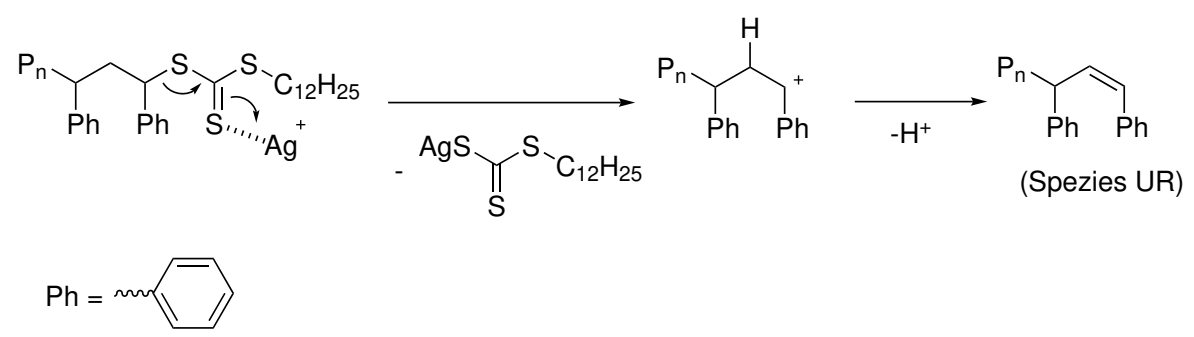

Schema 4.7. Möglicher E1 Mechanismus, der die Abspaltung der Trithiocarbonatgruppe und die Entstehung der Spezies UR erklärt.

Bei RAFT-PS ${ }_{2 k}$-Amin erfolgt die Ionisation ausschließlich über die Protonierung der Aminoendgruppe. Gegenüber der lonisation von RAFT-PS ${ }_{2 k}-\mathrm{COOH}$ mit Silberaddukten können so weiterhin Informationen über die Z-Gruppe erhalten werden. Es ist unklar, weshalb die Signale unterhalb von $\mathrm{m} / \mathrm{z}$ so intensiv sind. Diese treten bei RAFT-PS ${ }_{2 \mathrm{k}}-\mathrm{N}^{+}$, welches die gleiche MMV aufweist und sich nur durch die methylierte Ammoniumgruppe unterscheidet, nicht auf. Analog zu den PMA mit ionischer Endgruppe erfolgt bei RAFT-PS $\mathrm{Sk}_{2}-\mathrm{N}^{+}$die lonisation ausschließlich über die ionische Endgruppe und es werden keine Addukte gebildet.

Während bei PMA die ionische Endgruppe in der MALDI-MS zu wesentlich höheren Signalintensitäten und zu einem wesentlich höheren S/R-Verhältnis führt und es dadurch auch einfacher ist Spektren mit höherer Massenauflösung aufzunehmen, tritt dieser Effekt bei PS nicht auf. Wie die Abbildungen 4.25 A bis $\mathrm{C}$ zeigen, sind bei allen drei PS die Isotopenpeaks nicht vollständig voneinander getrennt. Wie Anhand von Tabelle 4.15 ersichtlich ist, ist bei dem PS mit ionischer Endgruppe die Erhöhung des S/R-Verhältnis gegenüber den anderen PS nur geringfügig.

Tab. 4.15. S/R-Verhältnis und die absoluten Signalintensitäten am Peakmaximum $\left(M_{\mathrm{p}}\right)$ der drei mittels MALDI-MS untersuchten Polystyrole.

\begin{tabular}{|c|c|c|}
\hline Polymer & Intensität $\left(M_{\mathrm{p}}\right) /$ a.u. & S/R-Verhältnis $\left(M_{\mathrm{p}}\right)$ \\
\hline 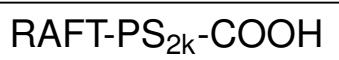 & 350 & 31 \\
\hline RAFT-PS $_{1 \mathrm{k}}-\mathrm{Amin}^{\mathrm{a}}$ & 5500 & 8,5 \\
\hline RAFT-PS $_{1 \mathrm{k}}-\mathrm{Amin}^{\bar{b}}$ & 1700 & 23 \\
\hline RAFT-PS ${ }_{2 k}-N^{+}$ & 13000 & 62 \\
\hline
\end{tabular}

${ }^{a} \mathrm{Am}$ Peak bei $653 \mathrm{~m} / \mathrm{z}$.

${ }^{b}$ Am Peak bei $2202 \mathrm{~m} / \mathrm{z}$. 


\subsection{Schlussfolgerung und Ausblick}

In diesem Kapitel konnten grundlegende Erkenntnisse über die massenspektrometrische Analyse von Polymeren gewonnen werden. Nach bestem Wissen des Autors wurden erstmalig Polymere mit und ohne ionische Endgruppen massenspektrometrisch untersucht und miteinander verglichen. Dazu wurde zunächst mit überwiegend neuen und literaturunbekannten RAFT-Agenzien PS und PMA mit variierenden Molmassen erfolgreich hergestellt, die wahlweise eine Carboxyl-, eine tertiäre Amino-, eine Ammonium- oder zwei Ammoniumgruppen in der Endgruppe enthalten.

Es stellte sich heraus, dass Polymere mit ionischer Endgruppe zur lonisation in der Massenspektrometrie weder eine Salz- noch eine Säurezugabe benötigen. Die ionische Endgruppe erhöhte die Signalintensität sowie das S/R-Verhältnis und die Sensitivität sowohl in der ESI- als auch in der MALDI-MS maßgeblich um einen Faktor von bis zu 140 gegenüber Polymeren ohne ionische Endgruppen. Lediglich in der MALDI-MS-Analyse von PS konnte bei Verwendung einer ionischen Gruppe das S/R-Verhältnis nur geringfügig erhöht werden. Die eingeführte ionische Gruppe könnte daher zukünftig von großem Wert in der Spurenanalytik von Polymeren oder im Nachweis von spurenhaltiger Spezies mittels MS sein. Vor allem in Verbindung mit der im ESI-Spektrometer einstellbaren Stoßenergie konnte durch die ionische Endgruppe auch bei PMA die Anzahl von Ladungen und die Ladungsverteilung reduziert werden. Dadurch wird die Interpretation der Massenspektren vereinfacht und die Anwendung der ESI-MS auf Polymere mit höherem Polymerisationsgrad erweitert. PS erzeugte in der ESI-MS auch bei Molmassen bis $4500 \mathrm{~g} \mathrm{~mol}^{-1}$ nur einfach geladene Ketten. Dies könnte an der geringeren Affinität von Polystyrol zur Komplexierung von Natrium liegen. Es wurde auch gezeigt, dass einer Kontrolle des Ladungszustandes in der ESI-MS zudem auch mit Polymeren möglich ist, die zwei Ammoniumgruppen enthalten. Die zwei kovalent eingebauten ionischen Gruppen erlauben die gezielte Bildung von zweifach geladenen Spezies in der ESI-MS, auch bei Polymerketten mit sehr kleinen Polymerisationsgraden. Dieses System könnte für Untersuchungen der Polymere mit der lonenmobilitäts-Massenspektrometrie von Interesse sein. In der MALDI-MS entstanden jedoch durch Neutralisation einer der beiden Ammoniumgruppen nur einfach geladenen Spezies.

Es konnten fast alle auftretenden Signale in der ESI- und MALDI-MS einer Spezies zugeordnet werden. Dabei traten in der MALDI-MS weniger Spezies auf, was u.a. am Auftreten von nur einfach geladenen Ketten und weniger Fragmentierungs-/Oxidationreaktionen liegen kann. Polymere mit protonierter tertiärer Aminoendgruppe ergaben in der ESI-MS jedoch sowohl bei PS als auch bei PMA mehrere unbekannte Spezies. Ab der Verwendung von fünf Äquivalenten Nal entstanden zudem in der PS- und PMA-ESI-MS-Analyse $\mathrm{Na}(\mathrm{Nal})_{x}$-Cluster und durch $\mathrm{Na}(\mathrm{Nal})_{x}$-Addukte ionisierte Polymerketten, wodurch eine Vielzahl neuer Signale im Massenspektrum auftraten. Daher muss die Konzentration an Nal für das jeweilige Polymer optimiert werden. Es konnte gezeigt werden, dass eine erhöhte Flussrate auch die Anzahl an mehrfach geladenen Ketten reduzieren und das S/R-Verhältnis erhöhen kann. Die Kapillarspannung ist ebenfalls ein wichtiger Geräteparameter, der optimiert werden muss, um hohe S/R-Verhältnisse zu erhalten. Jedoch führt eine erhöhte Kapillar- 
spannung ab $4 \mathrm{kV}$ zur Fragmentierung der labilen Trithiocarbonatgruppe. Für die Analyse von PS ist eine Adduktbildung in der MALDI-MS nur mit Silberionen möglich, welche jedoch die Trithiocarbonatgruppe abspalten. Daher ist für PS die vollständige Endgruppenbestimmung inklusive der Z-Gruppe nur von Polymeren möglich, die eine terminale Amino- oder Ammoniumendgruppe tragen.

In zukünftigen Studien könnte die Ammoniumgruppe auch in die Z-Gruppe der RAFT-Agenzien eingebaut werden. Dadurch ließen sich mithilfe des verbesserten S/RVerhältnisses explizit quasi lebende Polymerketten untersuchen, die die Z-Gruppe enthalten. Die intensiven und oft störenden Signale der HR Spezies würden dann nicht mehr detektiert. Zudem könnten auf Dithiobenzoaten basierende RAFT-Agenzien mit ionischen Gruppen hilfreich sein, die Crossterminierungsprodukte des intermediate radical termination Modells eindeutig und außerhalb von monomerfreien Modellsystemen nachzuweisen. 195-197] 


\section{Mechanische Eigenschaften reversibel photovernetzbarer Blockcopolymere}

\subsection{Motivation}

Die durch Mikrophasenseparation der Blockcopolymere (BCP) erhaltenen Nanostrukturen sind von großem Interesse und finden Anwendung in der Lithographie, als Nanostempel sowie als optische Schaltsensoren und Datenspeicher. ${ }^{[108-112}$ Darüber hinaus vereinen Blockcopolymere die Eigenschaften der jeweiligen Homopolymere, sodass die mechanischen Eigenschaften über das Verhältnis der Molmassen beider Blöcke präzise eingestellt werden können. So können aus Blockcopolymeren thermoplastische Elastomere hergestellt werden, die bei der Einsatztemperatur über den harten Block des Blockcopolymers physikalisch vernetzt sind. ${ }^{[5+7]}$ Klassische Elastomere entstehen durch irreversible weitmaschige chemische Vernetzung des bei der Anwendungstemperatur weichen Polymers. ${ }^{10[123]}$ Dies führt dazu, dass eine Wiedergewinnung des ursprünglichen Materials durch Recycling nicht möglich ist. Die chemische Vernetzung wird aber nicht nur zur Herstellung von Elastomeren eingesetzt, sie erhöht auch die Steifigkeit, die Härte sowie die thermische und chemische Stabilität des Werkstoffes. ${ }^{9110]}$ Eine reversible Vernetzung wäre daher ideal, um Elastomere und Duroplasten herzustellen, die nach ihrer Verwendung recycelt werden können.

Ziel dieses Projektes ist die Herstellung eines recycelbaren reversibel photovernetzbaren Blockcopolymers, dass aus einer weichen und einer harten Phase besteht. Dafür sollen mittels RAFT-Polymerisation Blockcopolymere aus Acrylaten und Methacrylaten hergestellt werden, die Mikrophasenseparation aufweisen und bei denen sich bei Raumtemperatur jeweils ein Block über- bzw. unterhalb der Glasübergangstemperatur befindet. Zudem soll ein Photovernetzer entweder in den weichen oder in den harten Block eingebaut werden, der über photochemische Cycloaddition mit einem weiteren Photovernetzer in der Polymerkette dimerisieren kann,. Der Einfluss der Photovernetzung auf die mechanischen Eigenschaften soll dabei zunächst für statistische Copolymere, einem vereinfachten System, anhand makroskopischer Probenkörper und der Verwendung des Zugversuches gezeigt werden. Die auf Cylcoadditionen basierende Photovernetzung in bulk und die damit verbundenen Änderungen der mechanischen Eigenschaften ist nach bestem Wissen des Autors in der Literatur noch nicht in bulk untersucht worden. Anschließend soll der Einfluss der selektiven Vernetzung innerhalb der weichen und innerhalb der harten Phase ebenfalls anhand des Zugversuches ermittelt werden. Auch eine solche Untersuchung ist nach bestem Wissen des Autors in der Literatur, auch anhand anderer Vernetzersysteme, noch nicht bekannt. Zudem soll die Reversibilität der Photovernetzung in dünnen Filmen mittels UV/VIS-Spektroskopie und in makroskopischen Probenkörpern anhand von Zugversuchen und Extraktionsversuchen bestimmt werden. 


\subsection{Verwendung von Cumarin als Photovernetzer}

\subsubsection{Synthese des Cumarinmonomers}

Zur Synthese des Cumarinmonomers wurde in einem kommerziell erhältlichen Derivat des Cumarins eine Methacrylateinheit eingeführt. Die Synthese des 7-(2-Methacryloyloxythoxy)4-methylcumarin (CMA) ist in Schema 5.1 dargestellt und wurde entsprechend der Literatur durchgeführt. [198] Zur Erhöhung der Flexibilität im späteren Polymer wurde der Abstand zwischen dem Cumarin und der Mathacrylsäurefunktionalität durch eine Ethylbrücke verlängert. Dafür wurde das Phenolderivat 7-Hydroxy-4-methylcumarin unter basischen Bedingungen mit 2-Chlorethanol zu 7-(2-Hydroxyethoxy)-4-methylcumarin umgesetzt. Im zweiten Schritt wurde die Monomerfunktionalität für die radikalische Polymerisation durch Veresterung von 7-(2-Hydroxyethoxy)-4-methylcumarin mit Methacylsäurechlorid eingeführt und so CMA erhalten. Die Struktur von CMA konnte durch NMR-Spektroskopie bestätigt werden. Die erhaltenen Spektren stimmen mit denen der Literatur überein. ${ }^{198}$

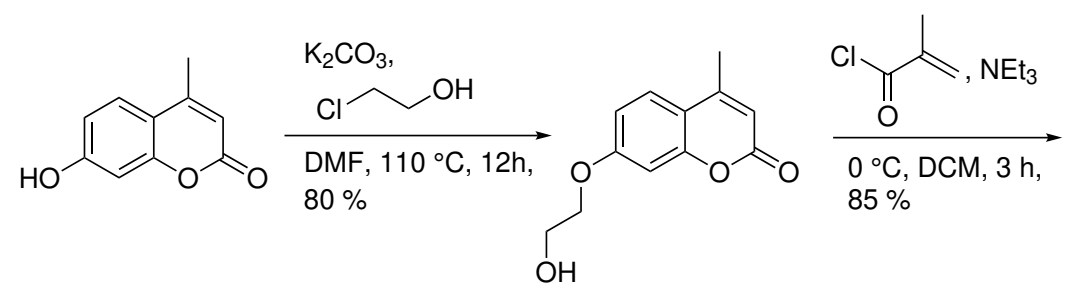

Schema 5.1. Synthese des Cumarinmonomers CMA.<smiles>C=C(C)C(=O)OCCOc1ccc2c(C)cc(=O)oc2c1</smiles>

CMA

\subsubsection{Synthese der photovernetzbaren cumarinhaltigen Polymere}

In diesem Abschnitt wird die Synthese der photovernetzbaren Blockcopolymere (BCP) auf Basis von CMA dargestellt. Dazu wird zunächst ein Block mittels RAFT-Polymerisation und unter Verwendung von 4-Cyano-4-[(dodecylsulfanylthiocarbonyl)sulfanyl]pentansäure (CDSPA, Abb. 5.2) als RAFT-Agens hergestellt. CDSPA ist besonders für die Polymerisation von Methacrylaten geeignet. ${ }^{3[169]}$ Es wird ein Makro-RAFT-Agens erhalten, welches anschließend mit einem weiteren Monomer zum Blockcopolymer umgesetzt wird (siehe auch Abb. 2.1).

Das BCP soll aus einem weichen Block mit einer Glasübergangstemperatur $T_{\mathrm{g}}$ unter $0{ }^{\circ} \mathrm{C}$ sowie einem harten Block mit einer Glasübergangstemperatur von über $45^{\circ} \mathrm{C}$ bestehen.

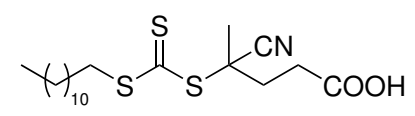

Schema 5.2. Struktur des verwendeten RAFT-Agens CDSPA. 
Dies gewährleistet die Zuordnung der einzelnen Phasen der Mikrophasenseparation in der Rasterkraftmikroskopie (engl. atomic force microscopy, AFM).

\section{Synthese der Makro-RAFT-Agenzien Poly(CMA-co-MMA)}

Die Synthese von CMA Copolymeren mit Methylmethacrylat (MMA) mittels RAFTPolymerisation wurde bereits ausführlich in der Literatur beschrieben. ${ }^{[199]}$ Dort wurde auch gezeigt, dass 5 mol\% CMA im harten Block ausreichend für eine Photovernetzung sind.

Aufbauend auf diesen Ergebnissen wurden verschiedene Makro-RAFT-Agenzien (Tabelle 5.1) hergestellt. Die RAFT-Polymerisationen einer Mischung aus 5 mol\% CMA und $95 \mathrm{~mol} \%$ MMA erfolgte unter Verwendung von AIBN als Initiator in Toluol. Die Makro-RAFT-Agenzien unterschiedlicher Molmassen wurden durch Variation des Verhältnisses Monomer (M) zu RAFT-Agens hergestellt.

Tab. 5.1. Erhaltene Molmassen und Dispersitäten der CMA/MMA-Copolymere mit 5 mol\% CMA. Die Gesamtmonomerkonzentration betrug 40 wt\% in Toluol.

\begin{tabular}{lcccc}
\hline Probe & M:RAFT:I & $M_{\mathrm{n}} /\left(\mathrm{g} \mathrm{mol}^{-1}\right)$ & $\oplus$ & $\mathrm{F}_{\mathrm{CMA}}{ }^{\natural} / \%$ \\
\hline P(MMM-co-CMA $)_{6 \mathrm{k}}$ & $50: 1: 0,1$ & 6,5 & 1,1 & 4,2 \\
P(MMM-co-CMA $)_{7 \mathrm{k}}$ & $50: 1: 0,1$ & 7,3 & 1,2 & 5,2 \\
P(MMM-co-CMA $)_{9 \mathrm{k}}$ & $100: 1: 0,2$ & 8,7 & 1,2 & 6,1 \\
\hline
\end{tabular}

${ }^{a}$ Molarer Anteil an CMA im Polymer bestimmt über ${ }^{1} \mathrm{H}-\mathrm{NMR}$-Spektroskopie.

Der Anteil an CMA im Polymer wurde über ${ }^{1} \mathrm{H}-\mathrm{NMR}$-Spektroskopie bestimmt. Dazu wurden bei MMA das Integral der Methylgruppe aus $-\mathrm{O}-\mathrm{CH}_{3}$ bei $\delta=3,61 \mathrm{ppm}$ und bei CMA das Integral der beiden Methylengruppen aus - $\mathrm{O}-\mathrm{CH}_{2}-\mathrm{CH}_{2}-\mathrm{O}$ - bei $\delta=4,19-4,43$ ppm verglichen. Die mittlere Molmassen $M_{\mathrm{n}}$ und die Dispersitäten $\Theta$ wurden mittels GPC anhand von PMMA-Kalibrierstandards bestimmt. Alle hergestellten Copolymere weisen eine geringe Dispersität und eine monomodale Molmassenverteilung (MMV) auf.

\section{Synthese der Poly(CMA-co-MMA)-b-Poly(BA) Blockcopolymere}

In der Literatur wurde bereits festgestellt, dass das BCP Poly(CMA-co-MMA)-b-Poly(BA) gut phasensepariert, vor allem wenn der Polybutylacrylat(PBA)-Block den längeren Block bildet. ${ }^{199}$ Des Weiteren erfüllt dieses System die Bedingung, dass das BCP einen harten [Poly(CMA-co-MMA)] und weichen (PBA) Block besitzt. Der ODT des BCPs soll bei einer Temperatur stattfinden, welche oberhalb der Temperatur bei der getempert wird und unterhalb von $200{ }^{\circ} \mathrm{C}$ liegt um mögliche Zersetzungs- oder Nebenreaktionen zu vermeiden. Dafür wurden Blockcopolymere mit möglichst kleiner Molmasse hergestellt, die dennoch eine MPS aufzeigen. Es wurden gezielt Polymere hergestellt, bei denen eine zylindrische Morphologie zu erwarten ist, da es anhand der zylindrischen Morphologie einfacher ist, den ODT zu beobachten. 


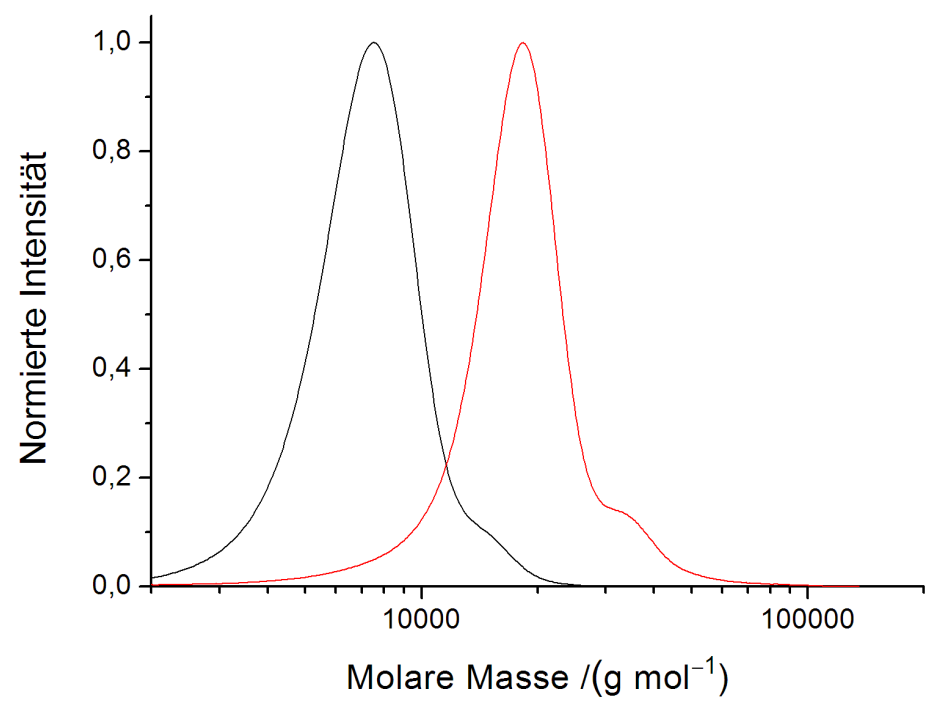

Abb. 5.1. Molmassenverteilungen des Makro-RAFT-Agens $P(M M A-c o-C M A)_{6 k}$ (schwarz) und des daraus hergestellten Blockcopolymers $\mathrm{P}(\mathrm{MMA}-\mathrm{co}-\mathrm{CMA})_{6 \mathrm{k}}-\mathrm{PBA}_{10 \mathrm{k}}$ (rot).

Ausgehend von den drei zuvor hergestellten Makro-RAFT-Agenzien wurden vier verschiedene Blockcopolymere mit unterschiedlich langen PBA-Blöcken hergestellt (Tabelle 5.2). Dazu wurde das Makro-RAFT-Agens und AIBN in einem Gemisch aus Butylacrylat und Toluol gelöst. Die Länge des PBA-Blockes konnte dabei durch die Variation der Polymerisationszeit oder des Verhältnisses von Monomer zu RAFT-Agens gesteuert werden. Der Anteil an BA und CMA wurde mittels ${ }^{1} \mathrm{H}-\mathrm{NMR}$-Spektroskopie bestimmt. Dazu wurden für MMA und CMA die Integrale der im vorherigen Abschnitt beschriebenen Signale verwendet. Für BA wurde das Integral der Methylengruppe aus $-\mathrm{O}-\mathrm{CH}_{2}$ - bei $\delta=4,04 \mathrm{ppm}$ verwendet. Die mittlere Molmasse $M_{\mathrm{n}}$ und die Dispersität $\emptyset$ wurde mittels GPC anhand von PMMA-Kalibrierstandards bestimmt. Die Dispersitäten steigen gegenüber der Dispersitäten der Makro-RAFT-Agenzien an, da der Anteil an Terminierungsprodukten zunimmt.

Abbildung 5.1 A und B zeigen beispielhaft die mittels GPC erhaltene MMV des Polymers $\mathrm{P}(\mathrm{MMM}-\mathrm{Co}-\mathrm{CMA})_{6 \mathrm{k}}-\mathrm{PBA}_{10 \mathrm{k}}$ sowie des Makro-RAFT-Agens, das zur Herstellung dieses $B C P$ verwendet wurde. Die Zunahme der Molmasse des Blockcopolymers gegenüber dem Makro-RAFT-Agens ist durch die Verschiebung des Kurvenmaximums zu erkennen (Abb. 5.1), was eine erfolgreiche Blockcopolymerisation bestätigt. Die Schulter bei höheren Molmassen entsteht vermutlich durch Terminierungsprodukte, deren Entstehung durch längere Polymerisationszeiten begünstigt werden. 
Tab. 5.2. Reaktionsbedingungen, Molmassen und Dispersitäten der Poly(MMA-co-CMA)- $b$ Poly(BA) Blockcopolymere.

\begin{tabular}{|c|c|c|c|c|c|c|}
\hline Probe & M:RAFT:I & $t / \mathrm{h}$ & $\begin{array}{c}M_{\mathrm{n}} \\
/\left(\mathrm{g} \mathrm{mol}^{-1}\right)\end{array}$ & $\theta$ & $\begin{array}{c}\mathrm{F}_{\mathrm{CMA}}{ }^{a} \\
/ \%\end{array}$ & $\begin{array}{c}\mathrm{F}_{\mathrm{BA}}{ }^{\mathrm{a}} \\
\%\end{array}$ \\
\hline $\mathrm{P}(\mathrm{MMA}-\mathrm{co}-\mathrm{CMA})_{6 \mathrm{k}}-\mathrm{PBA}_{10 \mathrm{k}}$ & $150: 1: 0,1$ & 1 & 16 & 1,2 & 2,3 & 59 \\
\hline$P(M M A-c o-C M A)_{7 k}-P A_{12 k}$ & $150: 1: 0,1$ & 22 & 20 & 1,3 & 2,0 & 64 \\
\hline $\mathrm{P}(\mathrm{MMA}-\mathrm{co}-\mathrm{CMA})_{9 \mathrm{k}}-\mathrm{PBA}_{15 \mathrm{k}}$ & $130: 1: 0,2$ & 20 & 24 & 1,3 & 2,1 & 66 \\
\hline$P(M M A-c o-C M A)_{9 k}-P B A_{16 k}$ & $150: 1: 0,2$ & 20 & 26 & 1,3 & 2,0 & 68 \\
\hline
\end{tabular}

${ }^{a}$ Anteil im Polymer bestimmt über ${ }^{1} \mathrm{H}-\mathrm{NMR}$-Spektroskopie.

\subsubsection{Bestimmung der Morphologie der Mikrophasenseparation}

Für die Photovernetzung der Blockcopolymere ist die Ausbildung einer Mikrophasenseparation essentiell, da der Einfluss der Vernetzung in der weichen und harten Phase untersucht werden soll. Um zu überprüfen, ob die BCP mikrophasenseparieren, wurden verdünnte Lösungen der Polymere auf Glasplättchen mittels spin coating hergestellt. Die Proben wurden anschließend im Ofen oberhalb der Glasübergangstemperatur beider Blöcke getempert, um eine ausreichende Beweglichkeit der Polymere und die Ausbildung der thermodynamischen Gleichgewichtsmorphologie zu gewährleisten. Die Rasterkraftmikroskopie (engl. atomic force microscopy, AFM) ermöglicht die Darstellung einer Oberflächentopographie und wird auch zur Untersuchung der MPS von Blockcopolymeren verwendet. 200-203]

Abbildung 5.2 zeigt die mittels AFM bei Raumtemperatur erhaltenen Höhenbilder der Blockcopolymere aus Tabelle 5.2. Bei P(MMA-co-CMA) 6k $^{-} \mathrm{PBA}_{10 \mathrm{k}}$ (Abb. 5.2 A), dem Polymer mit dem geringsten Polymerisationsgrad, entsteht eine nahezu planare Oberfläche ohne Struktur. In diesem Fall ist das Produkt aus Polymerisationsgrad und Flory-HugginsParamter so klein, dass beide Blöcke noch miteinander mischbar sind. Bei P(MMA-co$C M A)_{7 k}-P A_{12 k}$ wurde der Polymerisationsgrad leicht erhöht und es entsteht eine zylindrische Morphologie (Abb. 5.2 B). Das System befindet sich in der weak segregation region bzw. kurz oberhalb dieser, die Blöcke beginnen sich zu entmischen, aber es entsteht noch keine geordnete Struktur. Im Fall von P(MMA-co-CMA $)_{9 k}-P B_{16 k}$ und P(MMA-co-CMA $)_{9 k^{-}}$ $\mathrm{PBA}_{15 \mathrm{k}}$ (Abb. 5.2 C und D) wurde der Polymerisationsgrad weiter erhöht und es entsteht eine geordnete und zylindrische Morphologie, deren Höhenbild einem Fingerabdruck ähnelt. Dabei liegen die Zylinder parallel zur Oberfläche. Diese „Fingerabdruckstruktur“ könnte ebenfalls durch senkrecht zur Oberfläche liegenden Lamellen erzeugt werden. In der Literatur ist jedoch beschrieben, dass die hier untersuchten Blockcopolymere im Falle einer lamellaren MPS eine Insel-Loch-Morphologie mit Lamellen, die parallel zur Oberfläche liegen, ausbilden. Die dunklen Phasen können dem weichen PBA-Block zuzuordnen werden. 1992203 


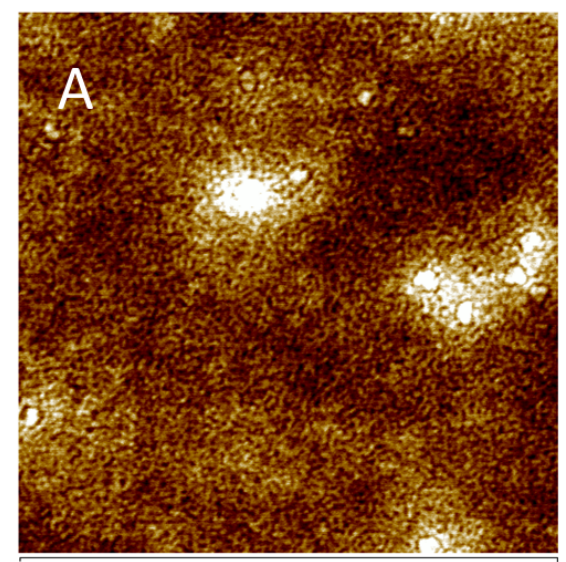

0,0

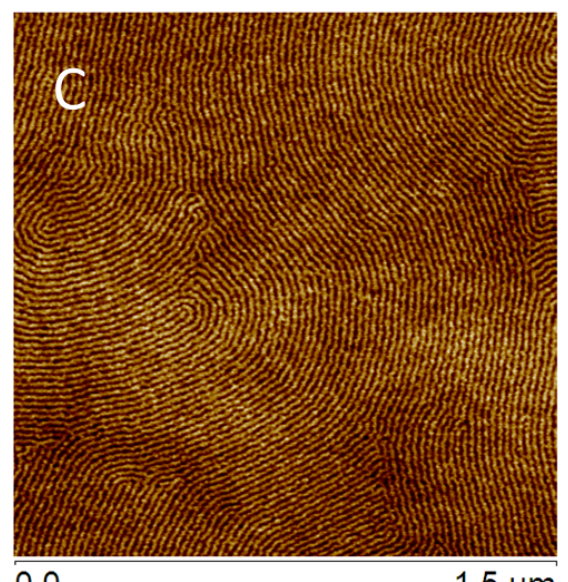

0,0
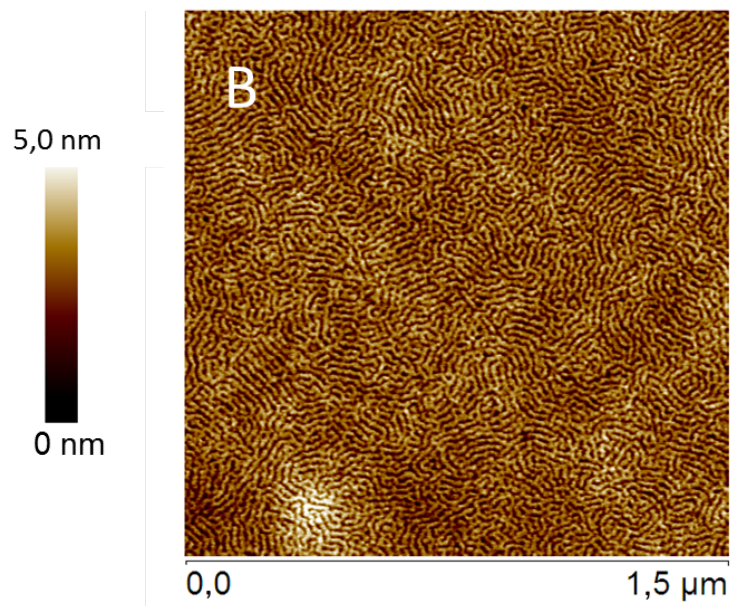

$9,4 \mathrm{~nm}$

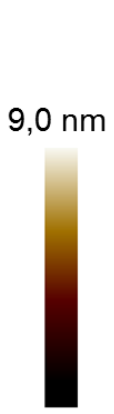

$0 \mathrm{~nm}$

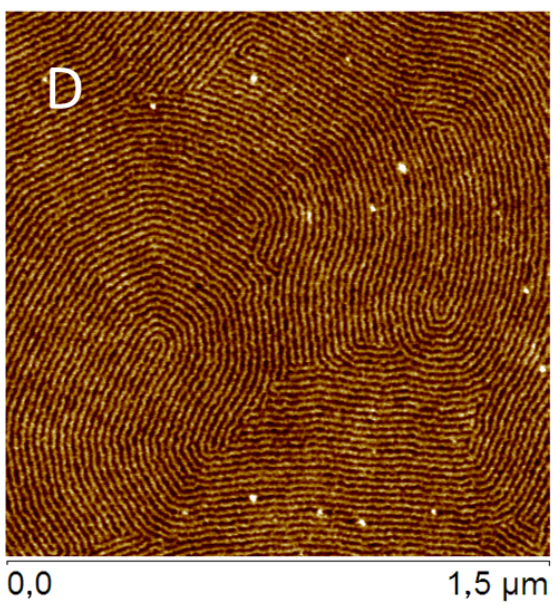

$9,0 \mathrm{~nm}$

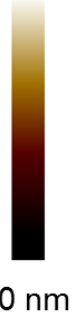

Abb. 5.2. Mittels AFM aufgenommene Höhenbilder der Blockcopolymere $P(M M A-c o-C M A)_{6 k}$ $\mathrm{PBA}_{10 \mathrm{k}}(\mathrm{A}), \mathrm{P}(\mathrm{MMA}-\mathrm{co}-\mathrm{CMA})_{7 \mathrm{k}}-\mathrm{PBA}_{12 \mathrm{k}}(\mathrm{B}), \mathrm{P}(\mathrm{MMA}-\mathrm{co}-\mathrm{CMA})_{9 \mathrm{k}}-\mathrm{PBA}_{15 \mathrm{k}}$ (C) und $\mathrm{P}(\mathrm{MMM}-\mathrm{Co}$ $\mathrm{CMA})_{9 \mathrm{k}}-\mathrm{PB}_{17 \mathrm{k}}(\mathrm{D})$. Die Auflösung aller Bilder beträgt $512 \times 512$ Pixel. 


\subsubsection{Untersuchung des Ordnungs-Unordnungs-Übergangs}

In diesem Abschnitt wird der Ordnung-Unordnung-Übergang (ODT) anhand der Probe $\mathrm{P}(\mathrm{MMA}-\mathrm{co}-\mathrm{CMA})_{9 k}-\mathrm{PBA}_{15 \mathrm{k}}$ untersucht, da dies die Probe mit der geringsten Molmasse ist, die eine geordnete zylindrische Morphologie aufweist. Je höher die Molmasse bzw. der Polymerisationsgrad, umso höher ist auch die Temperatur bei der der ODT auftritt. Der ODT soll aber bei einer möglichst niedrigen Temperatur oberhalb der zum Tempern benötigten Temperatur stattfinden.

Abbildung 5.3 A zeigt ein mittels AFM erhaltenes Höhenbild von P(MMA-co-CMA) ${ }_{9 k^{-}}$ $\mathrm{PBA}_{15 \mathrm{k}}$ mit einer zylindrischen Morphologie. Im Innenbild ist die spectral density Fourier Transformation des Höhenbilds gezeigt. Neben dem Maximum in der Mitte (hell) gibt es ein weiteres, ringförmiges Maximum. Dies bestätigt, dass eine geordnete Struktur ohne Vorzugsrichtung vorhanden ist. Abbildung 5.3 B zeigt das Höhenbild der Probe, nachdem sie

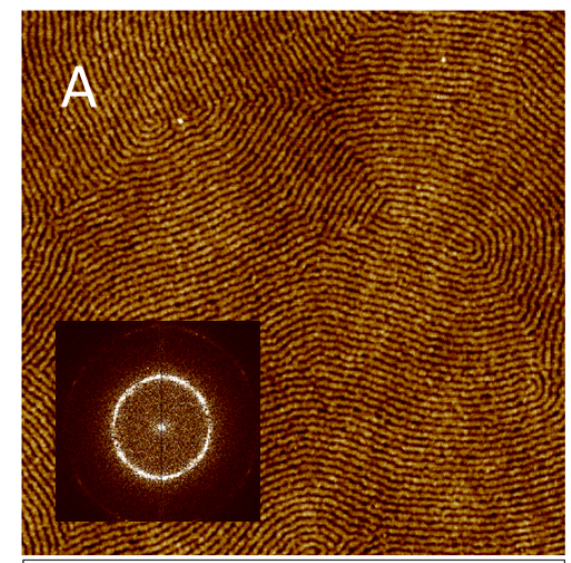

0,0

$1,5 \mu \mathrm{m}$
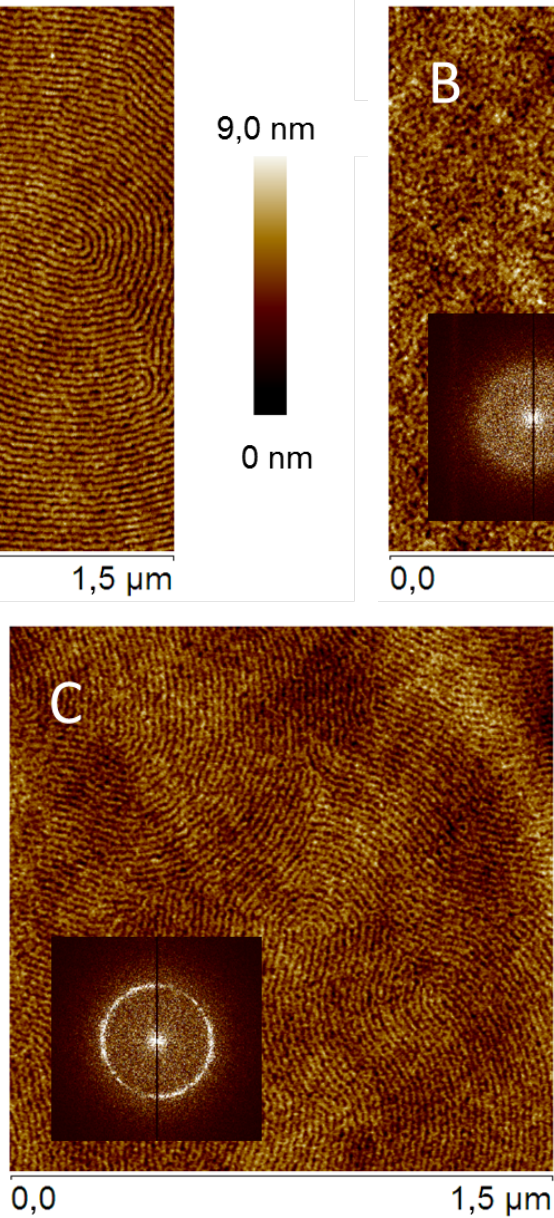

$6,3 \mathrm{~nm}$

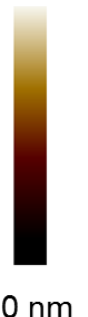

Abb. 5.3. Mittels AFM aufgenommene Höhenbilder des Blockcopolymers $P(M M A-c o-C M A)_{9 k^{-}}$ $\mathrm{PBA}_{15 \mathrm{k}}$ nach Tempern bei $140{ }^{\circ} \mathrm{C}(\mathrm{A})$ und $195{ }^{\circ} \mathrm{C}(\mathrm{B})$. Die Probe in Abb. C wurde nach dem Tempern bei $140^{\circ} \mathrm{C}$ bei Raumtemperatur photovernetzt und dann auf $195^{\circ} \mathrm{C}$ erwärmt. Alle Messungen fanden bei Raumtemperatur statt. Die Innenbilder zeigen die spectral density Fourier Transformation der jeweiligen Höhenbilder. Auflösungen: 512 x 512 Pixel. 
bei $195^{\circ} \mathrm{C}$ getempert und schnell auf Raumtemperatur abgekühlt wurde. Die zylindrische Struktur ist nicht mehr vorhanden und auch die spectral density Fourier Transformation weist kein zweites Maximum mehr auf. Daraus lässt sich schließen, dass ein ODT stattgefunden hat. Der ODT ist ein reversibler Übergang, dass heißt nach Erhitzen auf $195^{\circ} \mathrm{C}$ sollte sich die zylindrische Struktur wieder durch Tempern bei $140^{\circ} \mathrm{C}$ ausbilden. Im Falle der hier untersuchten Probe war dieser Vorgang jedoch nicht zu erkennen. Möglicherweise treten bei $195^{\circ} \mathrm{C}$ Nebenreaktionen auf, die das Polymer beschädigen oder verändern. Eine Untersuchung der Probe mittels thermogravimetrische Analyse (TGA) ergab, dass bis $250{ }^{\circ} \mathrm{C}$ nur ein Massenverlsut von etwa $1 \%$ auftritt (Abb. 5.4). Eine Degradierung oder Depolymerisation des Polymers findet demnach bei $195^{\circ}$ nicht statt. In Abbildung 5.3 C wurde die Probe nach dem Tempern bei $140^{\circ} \mathrm{C}$ bei Raumtemperatur photochemisch für drei Stunden vernetzt. Anschließend wurde die vernetzte Probe erneut bei $195^{\circ} \mathrm{C}$ getempert und schnell auf Raumtemperatur abgekühlt. Die zylindrische Struktur bleibt erhalten, was auch die spectral density Fourier Transformation im Innenbild bestätigt. Die Probe konnte somit erfolgreich vernetzt werden und die MPS eingefroren werden.

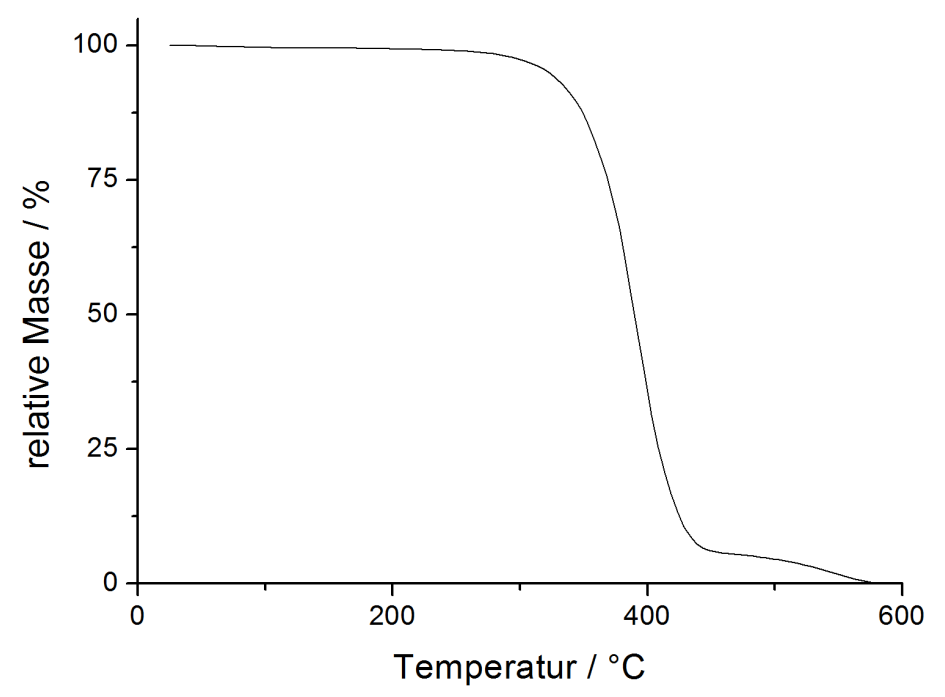

Abb. 5.4. TGA-Kurve des Polymers $P(M M A-c o-C M A)_{9 k}-P_{B A} A_{15 k}$ gemessen in Luft und mit einer Heizrate von $10 \mathrm{~K} \mathrm{~min}^{-1}$.

\subsubsection{Untersuchung der reversiblen Photovernetzung in dünnen Filmen}

Die reversible Photovernetzung der Cumarin-Polymere wird anhand der Probe P(MMA-co$\mathrm{CMA})_{7 \mathrm{k}}$ untersucht. Dazu wurde ein dünner Film der Polymerlösung durch drop casting auf einem Quarzglasplättchen hergestellt und mittels UV/VIS-Spektroskopie untersucht.

Abbildung 5.5 A zeigt ein UV/VIS-Spektrum des hergestellten Polymerfilms. Während der Bestrahlung mit $h \nu>305 \mathrm{~nm}$ sinkt die Absorption (schwarzer Pfeil) der Bande, da die Probe vernetzt und das Cumarindimer nicht mehr in dem untersuchten Wellenlängenbereich absorbiert. Die Vernetzung benötigt relativ lange Bestrahlzeiten, da die Bestrahlung bei Raumtemperatur und unterhalb des $T_{\mathrm{g}}$ des Polymers stattfindet. Nach vier Stunden Vernetzung 
wurde die Probe mittels Bestrahlung mit $h \nu=254 \mathrm{~nm}$ entnetzt, wodurch die Absorption wieder leicht ansteigt (roter Pfeil). Die Entnetzung der Probe ist jedoch wesentlich ineffizienter als die Vernetzung. Möglicherweise führt die lange Bestrahldauer während der Vernetzung zu Nebenreakionen. Daher wurde ein zweiter Film für die Vernetzung nur für 3 Minuten bestrahlt bzw. vernetzt und dann für 40 Sekunden mit Bestrahlung der kürzeren Wellenlänge entnetzt. Die Ver- und Entnetzung des Polymers wurde über mehrere Zyklen durchgeführt und ist in Abbildung $5.5 \mathrm{~B}$ dargestellt. Dabei bedeutet eine sinkende Absorption, dass die Probe mittels $h \nu>305 \mathrm{~nm}$ vernetzt wurde, und eine steigende Absorption, dass die Probe mittels $h \nu=254 \mathrm{~nm}$ entnetzt wurde. Mit der kürzeren Bestrahlzeit für die Vernetzung nimmt die Effizienz der Entnetzung zu. Allerdings wird selbst im ersten Zyklus weniger als die Hälfte der Vernetzungsstellen wieder entnetzt und mit zunehmender Anzahl an Zyklen sinkt die Effizienz der Entnetzung weiter. Der CMA Photovernetzer ist aufgrund seiner ineffizienten Entnetzung nicht geeignet, die reversible Vernetzung in makroskopischen Probenkörpern zu untersuchen.
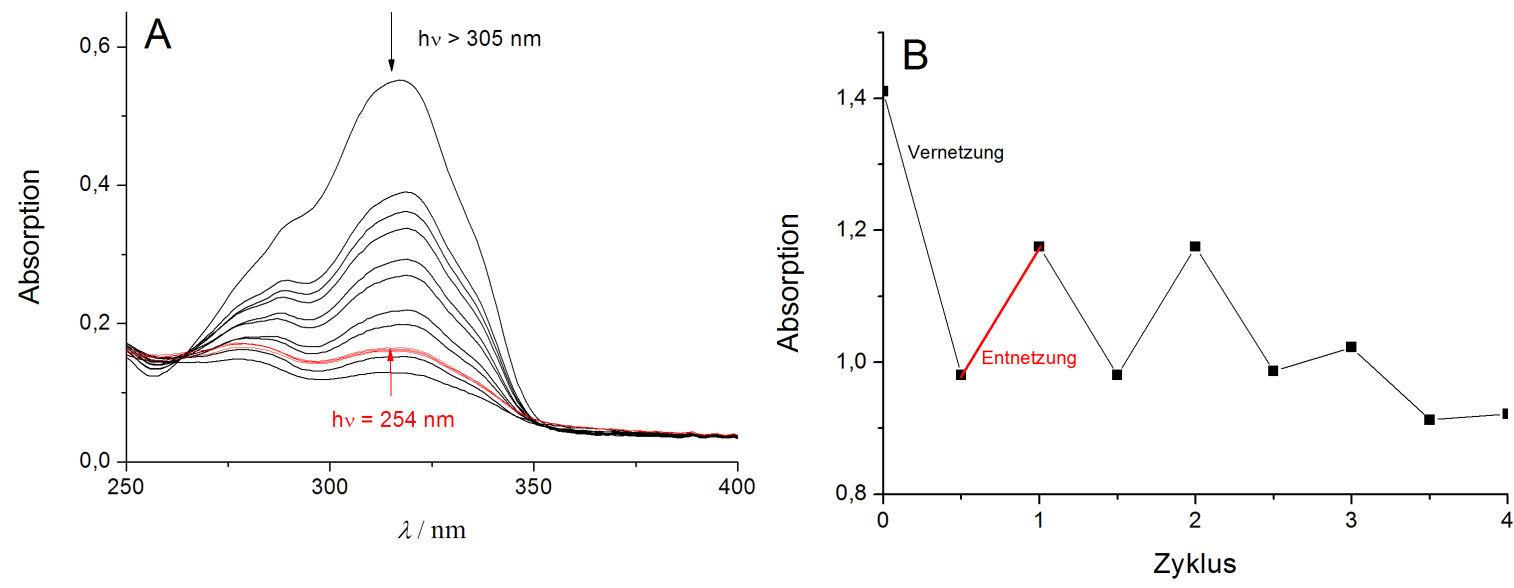

Abb. 5.5. A: UV/VIS-Spektrum des Cumarin enthaltenden Polymers $P(M M M-c o-C M A)_{7 k}$. Der schwarze Pfeil gibt die Entwicklung der Bande mit Bestrahlung mit $h \nu>305 \mathrm{~nm}$ an. Der rote Pfeil gibt die Entwicklung der Bande mit der niedrigsten Absorption bei Bestrahlung mit $h \nu=254 \mathrm{~nm}$ an. B: Entwicklung der Absorption am Maximum bei $319 \mathrm{~nm}$ über mehrere Zyklen der Ver- und Entnetzung. 


\subsection{Verwendung von Anthracen als Photovernetzer}

Da sich herausstellte, dass das CMA als Photovernetzer ungeeignet ist, wurde ein neuer Photovernetzer auf Basis des Anthracens verwendet. Bei Verwendung des Anthracens können die photochemisch erzeugten Vernetzungsstellen auch thermisch wieder aufgelöst werden (siehe auch Abschnitt 2.6. [141] Die ist laut Literatur effektiver als die photochemische Entnetzung. $137|138| 141 \mid 145]$ Zudem ist die photochemische Entnetzung bei dickeren Proben zusätzlich dadurch eingeschränkt, dass die benötigte kurzwellige Strahlung auch durch viele weitere Gruppen im Polymer absorbiert wird. Diese Einschränkung entfällt ebenfalls bei der thermischen Entnetzung des Anthracenphotovernetzers.

\subsubsection{Synthese des Anthracenmonomers}

Basierend auf Anthracen wurde 9-Anthracenylmethylmethacrylat (ANMA) als weiterer Photovernetzer entsprechend Schema 5.3 und der Literatur hergestellt. 204] Dazu wurde 9Hydroxymethylanthracen mit Methacrylsäurechlorid mit einer Ausbeute von $90 \%$ zu ANMA verestert. Die Struktur und ANMA konnte durch NMR-Spektroskopie bestätigt werden. Die erhaltenen Spektren stimmen mit denen der Literatur überein. 204

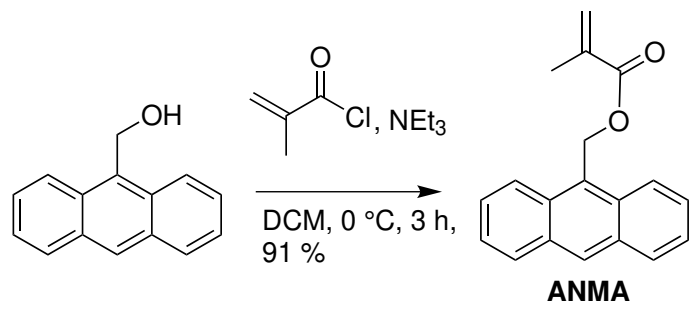

Schema 5.3. Synthese des Anthracenmonomers ANMA.

\subsubsection{Synthese der photovernetzbaren anthracenhaltigen Polymere}

Bei den anthracenhaltigen Polymeren wurde sowohl für den weichen als auch für den harten Block ein Methacrylatmonomer gewählt, da Anthracen die Polymerisation von Acrylaten inhibiert. 139[142] Um den Einfluss der Photovernetzung im weichen und harten Block zu untersuchen, wurde das ANMA jeweils in die entsprechenden Blöcken eingebaut. Als Monomer für den weichen Block wurde Laurylmethacrylate (LMA) mit einem $T_{\mathrm{g}}$ von etwa -48 bis $-65^{\circ} \mathrm{C}$ verwendet. 205/206 Als Monomer für den harten Block wurde Butylmethacrylat (BMA) mit einem $T_{\mathrm{g}}$ von etwa 35 bis $50{ }^{\circ} \mathrm{C}$ verwendet. 207/208] Die Glasübergangstemperatur von Poly(Butylmethacrylate) (PBMA) liegt nur knapp über Raumtemperatur und benötigt daher ein Comonomer zur Erhöhung des $T_{\mathrm{g}}$. Das Comonomer ist entweder der Photovernetzer ANMA oder MMA, wenn ANMA in dem weichen Block eingebaut wurde. 


\section{Synthese der statistischen Copolymere und Makro-RAFT-Agenzien}

Es wurden statistische Copolymere von ANMA mit den Monomeren des weichen Blocks (LMA) und des harten Blocks (BMA) hergestellt, um den Einfluss der reversiblen Photovernetzung auf die mechanischen Eigenschaften dieser statistischen Copolymere zu untersuchen. Im weichen Block wurde zudem der Anteil von ANMA variiert, um auch den Einfluss der ANMA-Konzentration während der Photovernetzung bestimmen zu können.

Tab. 5.3. Erhaltene Molmassen, Dispersitäten und Zusammensetzungen der statistischen Copolymere aus ANMA und LMA bzw. BMA.

\begin{tabular}{|c|c|c|c|c|c|}
\hline Probe & $\begin{array}{c}M_{\mathrm{n}} \mathrm{a}^{-1} \\
/\left(\mathrm{g} \mathrm{mol}^{-1}\right)\end{array}$ & $\begin{array}{l}M_{\mathrm{n}, \mathrm{th}}{ }^{b} \\
/\left(\mathrm{g} \mathrm{mol}^{-1}\right)\end{array}$ & 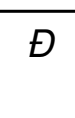 & $\begin{array}{c}\mathrm{f}_{\text {ANMA }}{ }^{C} \\
/ \%\end{array}$ & $\begin{array}{c}\mathrm{F}_{\text {ANMA }}{ }^{a} \\
/ \%\end{array}$ \\
\hline $\mathrm{P}\left(\mathrm{LMA}_{0,85}-\mathrm{Co}-\mathrm{ANMA}_{0,15}\right)$ & 49 & 62 & 1,1 & 16 & 15 \\
\hline $\mathrm{P}\left(\mathrm{LMA}_{0,92}-\mathrm{Co}-\mathrm{ANMA}_{0,08}\right)$ & 48 & 69 & 1,2 & 9,1 & 8 \\
\hline $\mathrm{P}\left(\mathrm{LMA}_{0,95}-\mathrm{Co}-\mathrm{ANMA}_{0,05}\right)$ & 52 & 72 & 1,2 & 4,8 & 5 \\
\hline $\mathrm{P}\left(\mathrm{BMA}_{0,9}-\right.$ co-ANMA $\left.\mathrm{A}_{0,1}\right)$ & 36 & 36 & 1,2 & 10 & 10 \\
\hline
\end{tabular}

\footnotetext{
a'Bestimmt mit PMMA-Kalibrierstandards.

${ }^{b}$ bestimmt über den Umsatz und Gleichung 4.1

${ }^{c}$ Anteil ANMA im Polymeransatz.

${ }^{d}$ Anteil ANMA im Polymer bestimmt über ${ }^{1} \mathrm{H}$-NMR-Spektroskopie.
}

Die statistischen Copolymere aus LMA bzw. BMA und ANMA werden im Folgenden entsprechend ihrer Zusammensetzung statt der Molmasse benannt. Dabei bezeichnet das tiefgestellte $x$ den molaren Anteil des jeweiligen Blocks.

Durch Variation der Konzentration an ANMA im Polymerisationsansatz konnten statistische Copolymere mit unterschiedlichen Anteilen an ANMA im Polymer erhalten werden (Tabelle 5.3). Dabei entsprechen die eingesetzten Konzentrationen von ANMA in etwa dem Anteil des eingebauten ANMA im resultierenden Polymer. Die experimentellen Molmassen sind geringer als die anhand von Gleichung 4.1 bestimmten theoretischen Molmassen. Dies ist auf die Verwendung einer PMMA-Kalibrierung der GPC zurückzuführen, sodass die experimentell bestimmten Molmassen lediglich als Anhaltspunkt für die ungefähre Molmassen dient. Bei dem statistischen Copolymer aus BMA und ANMA stimmt die experimentell bestimmte Molmasse gut mit der theoretisch berechnete Molmasse überein. Alle Polymere weisen eine monomodale MMV mit niedriger Dispersität zwischen 1,1 und 1,2 auf.

Zur Bestimmung des ANMA-Anteils wurde bei LMA bzw. BMA das Integral der Methylengruppe aus $-\mathrm{O}-\mathrm{CH}_{2}$ - bei $\delta=3,90 \mathrm{ppm}$ sowie bei $\delta=6,02 \mathrm{ppm}$ für ANMA verwendet. Bei ANMA können alternativ auch die Signale der aromatischen Protonen verwendet werden. Beispielhaft ist ein ${ }^{1} \mathrm{H}-\mathrm{NMR}$-Spektrum von $\mathrm{P}\left(\mathrm{LMA}_{0,83}-\mathrm{Co}^{\left.-\mathrm{ANMA}_{0,17}\right)}\right.$ mit der Zuordnung der für die Bestimmung der Zusammensetzung wichtigen Signale in Abbildung 5.6 gezeigt.

Des Weiteren wurden Makro-RAFT-Agenzien bestehend aus LMA, LMA und ANMA sowie BMA und ANMA hergestellt (Tabelle 5.4). Diese werden im Folgenden zur Synthese von Blockcopolymeren verwendet. Die Diskussion der Synthesen der Makro-RAFT-Agenzien ist analog zu den Synthesen der zuvor besprochenen statistischen Copolymere aus Tabelle 5.3 . 


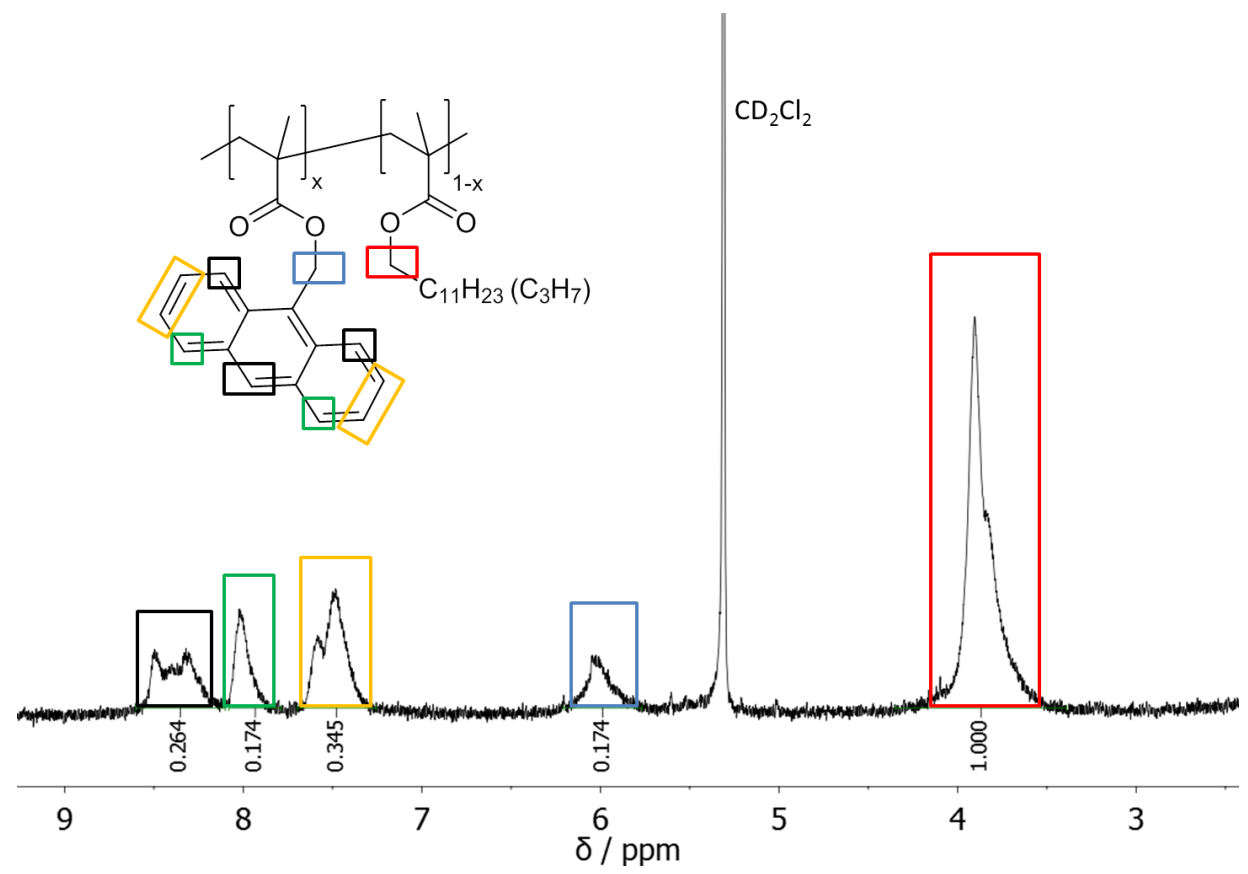

Abb. 5.6. ${ }^{1} \mathrm{H}-\mathrm{NMR}-\mathrm{Spektrum}$ von $\mathrm{P}\left(\mathrm{LMA}_{0,85}-\mathrm{Co}-\mathrm{ANMA}_{0,15}\right)$. Für die Bestimmung der Zusammensetzung wurde das rot und blau markierte Signal verwendet. Statt dem blau markierten Signal können aber auch die aromatischen Signale verwendet werden.

Tab. 5.4. Reaktionsbedingungen, Molmassen, Dispersitäten und Zusammensetzungen der Makro-RAFT-Agenzien. Alle Polymerisationen wurden bei $60^{\circ} \mathrm{C}$ durchgeführt.

\begin{tabular}{|c|c|c|c|c|c|}
\hline Probe & Monomer & $t / \mathrm{h}$ & $\begin{array}{l}M_{\mathrm{n}}{ }^{a} \\
/\left(\mathrm{g} \mathrm{mol}^{-1}\right)\end{array}$ & 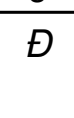 & $\begin{array}{c}\mathrm{F}_{\text {ANMA/MMA }} \\
\%\end{array}$ \\
\hline $\mathrm{P}\left(\mathrm{BMA}-\mathrm{co}-\mathrm{MMA}_{10 \mathrm{k}}\right)$ & $\begin{array}{l}20 \% \text { BMA, } \\
80 \% \text { MMA }\end{array}$ & 13,5 & 10 & 1,2 & $20^{b}$ \\
\hline $\mathrm{PLMA}_{21 \mathrm{k}}$ & $100 \%$ LMA & 14 & 21 & 1,2 & - \\
\hline$P\left(\right.$ LMA-co-ANMA $\left.{ }_{21 \mathrm{k}}\right)$ & $\begin{array}{l}90 \% \text { LMA, } \\
10 \% \text { ANMA }\end{array}$ & 14,5 & 21 & 1,2 & $12^{c}$ \\
\hline $\mathrm{P}(\text { LMA-co-ANMA })_{52 \mathrm{k}}$ & $\begin{array}{l}95 \% \text { LMA, } \\
5 \% \text { ANMA }\end{array}$ & 17 & 60 & 1,2 & $4,6 \mathrm{C}$ \\
\hline
\end{tabular}

${ }^{a}$ Bestimmt mittels PMMA-Kalibrierung.

${ }^{b}$ Im Polymer eingebauter Anteil an MMA.

${ }^{c}$ Im Polymer eingebauter Anteil an ANMA. 


\section{Synthese der ANMA enthaltenden Poly(LMA)-b-Poly(BMA) Blockcopolymere}

Ausgehend von den Makro-RAFT-Agenzien aus Tabelle 5.4 wurden Poly(LMA)-b-Poly(BMA) Blockcopolymere hergestellt. ANMA ist entweder in dem BMA, LMA oder in beiden Blöcken eingebaut. Die Polymerisationen wurden bei $60^{\circ} \mathrm{C}$ durchgeführt.

In Tabelle 5.5 sind die erhaltenen Blockcopolymere mit den unterschiedlichen Reaktionsbedingungen und den resultierenden Molmassen zusammengefasst. Mittels des Verhältnisses von Monomer zu RAFT-Agens und variierender Polymerisationszeiten konnten erfolgreich Blockcopolymere mit unterschiedlichen Molmassen hergestellt werden. Es wurden geringe Dispersitäten zwischen 1,1 und 1,2 erhalten. Lediglich die Dispersität von P(LMAco-ANMA) ${ }_{79 k}$ )-PBMA ${ }_{21 k}$ ist etwas höher. Die Molmassen nehmen gegenüber den ersten Block zu, wie Abbildung 5.7 anhand von P(BMA-co-MMA) ${ }_{10 \mathrm{k}}-\mathrm{P}(\mathrm{LMA}-\text { co-ANMA })_{19 \mathrm{k}}$ beispielhaft zeigt.

Tabelle 5.6 zeigt die Zusammensetzungen der Polymerisationslösungen und den daraus resultierenden Anteilen der Comonomere in den Bockcopolymeren. Für P(BMA-coMMA $)_{10 \mathrm{k}}-\mathrm{P}(\text { LMA-Co-ANMA) })_{19 \mathrm{k}}$ konnte der Anteil an MMA und ANMA mittels ${ }^{1} \mathrm{H}-\mathrm{NMR}$ Spektroskopie direkt aus den jeweiligen Integralen bestimmt werden. Hierzu dienten die in Abbildung 5.8 markierten Signale. Das Signal bei $\delta=3,90 \mathrm{ppm}$ setzt sich aus den Protonen des BMA und LMA aus - $\mathrm{O}-\mathrm{CH}_{2}$ zusammen. Aus dem berechneten Anteil des MMA und dem bekannten Verhältnis von MMA zu BMA (siehe Tabelle 5.4 konnte der Anteil an BMA und folglich auch LMA berechnet werden. Analog konnte bei P(LMA-co-ANMA) $49 k$ )$\mathrm{PBMA}_{21 \mathrm{k}}$ aus dem bekannten Verhältnis von LMA zu ANMA im ersten Block auch der Anteil von LMA und BMA im Gesamtpolymer bestimmt werden.

Für die weiteren Blockcopolymere konnten lediglich der Anteil des ANMA im Blockcopolymer mittels ${ }^{1} \mathrm{H}$-NMR-Spektroskopie ermittelt werden. Daraus und unter Verwendung des Massenzuwachses aus den GP-Chromatogrammen konnten die Anteile an BMA und LMA im Gesamtpolymer abgeschätzt werden. Bei den Polymeren die aus dem selben MakroRAFT-Agens hergestellt wurden, konnte über die Polymerisationszeit der Anteil an BMA kontrolliert werden, was mit der Zunahme der Molmasse aus Tabelle 5.5 übereinstimmt.

Tab. 5.5. Reaktionsbedingungen, Molmassen und Dispersitäten der ANMA enthaltenden Poly(LMA)- $b$-Poly(BMA) Blockcopolymere. Alle Polymerisationen wurden bei $60^{\circ} \mathrm{C}$ durchgeführt.

\begin{tabular}{|c|c|c|c|c|}
\hline Probe & M:RAFT:I & $t / \mathrm{h}$ & $\begin{array}{c}M_{\mathrm{r}}{ }^{a} \\
/\left(\mathrm{g} \mathrm{mol}^{-1}\right)\end{array}$ & 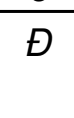 \\
\hline $\mathrm{P}(\mathrm{BMA}-\text { co-MMA })_{10 k}-\mathrm{P}(\text { LMA-co-ANMA })_{19 \mathrm{k}}$ & $90: 1: 0,1$ & 14 & 29 & 1,1 \\
\hline $\mathrm{PLMA}_{21 \mathrm{k}}-\mathrm{P}(\mathrm{BMA}-\mathrm{Co}-\mathrm{ANMA})_{11 \mathrm{k}}$ & $180: 1: 0,1$ & 14 & 32 & 1,1 \\
\hline $\mathrm{PLMA}_{21 \mathrm{k}}-\mathrm{P}(\mathrm{BMA}-\mathrm{co}-\mathrm{ANMA})_{17 \mathrm{k}}$ & $180: 1: 0,1$ & 18 & 38 & 1,1 \\
\hline $\mathrm{PLMA}_{21 \mathrm{k}}-\mathrm{P}(\mathrm{BMA}-\mathrm{Co}-\mathrm{ANMA})_{21 \mathrm{k}}$ & $180: 1: 0,1$ & 22 & 42 & 1,2 \\
\hline $\mathrm{P}(\text { LMA-co-ANMA })_{21 \mathrm{k}}-\mathrm{P}(\mathrm{BMA}-\mathrm{co}-\mathrm{ANMA})_{10 \mathrm{k}}$ & $180: 1: 0,1$ & 14 & 31 & 1,2 \\
\hline $\mathrm{P}(\text { LMA-co-ANMA })_{21 \mathrm{k}}-\mathrm{P}(\mathrm{BMA}-\mathrm{co}-\mathrm{ANMA})_{27 \mathrm{k}}$ & $220: 1: 0,1$ & 24 & 48 & 1,2 \\
\hline $\left.\mathrm{P}(\text { LMA-co-ANMA })_{49 \mathrm{k}}\right)-\mathrm{PBMA}_{21 \mathrm{k}}$ & $150: 1: 0,2$ & 15 & 70 & 1,4 \\
\hline
\end{tabular}

${ }^{a}$ Bestimmt mittels PMMA-Kalibrierung. 


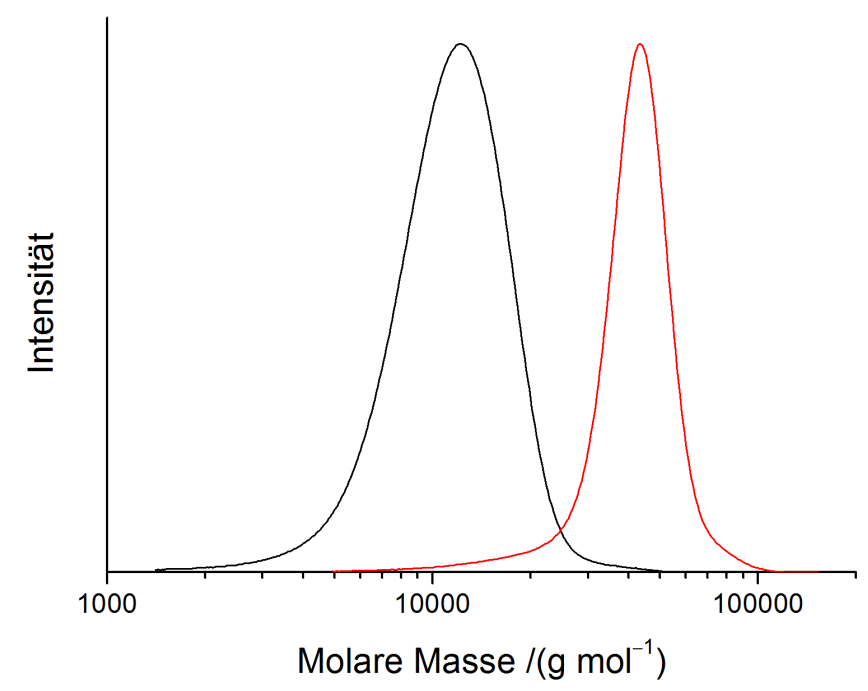

Abb. 5.7. Die durch GPC bestimmte Molmassenverteilung des $P(B M A-c o-M M A)_{10 k}-P(L M A-c o-$ ANMA) $_{19 k}$ (rot) sowie des Makro-RAFT-Agens (erster Block, schwarz), das zur Herstellung des Blockcopolymers verwendet wurde.

Tab. 5.6. Die berechneten Zusammensetzungen der ANMA enthaltenden Poly(LMA)- $b$ Poly(BMA) Blockcopolymere.

\begin{tabular}{|c|c|c|c|c|}
\hline Probe & Monomera & $\begin{array}{c}\mathrm{F}_{\text {ANMA }} \\
\%\end{array}$ & $\begin{array}{c}\mathrm{F}_{\mathrm{LMA}} \\
\%\end{array}$ & $\begin{array}{c}\mathrm{F}_{\mathrm{BMA}} \\
\%\end{array}$ \\
\hline $\mathrm{P}(\mathrm{BMA}-\mathrm{co}-\mathrm{MMA})_{10 \mathrm{k}}-\mathrm{P}(\text { LMA-co-ANMA })_{19 \mathrm{k}}$ & $\begin{array}{l}95 \% \text { LMA, } \\
5 \% \text { ANMA }\end{array}$ & 3,4 & 44,6 & $41,5^{b}$ \\
\hline $\mathrm{PLMA}_{21 \mathrm{k}}-\mathrm{P}(\mathrm{BMA}-\mathrm{co}-\mathrm{ANMA})_{11 \mathrm{k}}$ & $\begin{array}{l}90 \% \text { BMA, } \\
9 \% \text { ANMA }\end{array}$ & 2,3 & 48 & 50 \\
\hline $\mathrm{PLMA}_{21 \mathrm{k}}-\mathrm{P}(\mathrm{BMA}-\mathrm{Co}-\mathrm{ANMA})_{17 \mathrm{k}}$ & $\begin{array}{l}90 \% \text { BMA, } \\
9 \% \text { ANMA }\end{array}$ & - & 36 & 62 \\
\hline $\mathrm{PLMA}_{21 \mathrm{k}}-\mathrm{P}(\mathrm{BMA}-\mathrm{co}-\mathrm{ANMA})_{21 \mathrm{k}}$ & $\begin{array}{l}90 \% \text { BMA, } \\
9 \% \text { ANMA }\end{array}$ & 2,8 & 31 & 66 \\
\hline $\mathrm{P}(\text { LMA-co-ANMA })_{21 \mathrm{k}}-\mathrm{P}(\mathrm{BMA}-\text { co-ANMA })_{10 \mathrm{k}}$ & $\begin{array}{l}90 \% \text { BMA, } \\
10 \% \text { ANMA }\end{array}$ & 9,1 & 47 & 44 \\
\hline $\mathrm{P}(\text { LMA-co-ANMA })_{21 \mathrm{k}}-\mathrm{P}(\mathrm{BMA}-\text { co-ANMA })_{27 \mathrm{k}}$ & $\begin{array}{l}90 \% \text { BMA, } \\
10 \% \text { ANMA }\end{array}$ & 8,7 & 41 & 50 \\
\hline $\left.\mathrm{P}(\mathrm{LMA}-\mathrm{co}-\mathrm{ANMA})_{49 \mathrm{k}}\right)-\mathrm{PBMA}_{21 \mathrm{k}}$ & BMA & 8 & 45 & 47 \\
\hline
\end{tabular}

${ }^{a}$ Eingesetzte Monomere im Reaktionsansatz des zweiten Blocks.

${ }^{b}$ Dieses Polymer enthält noch $10,5 \%$ an MMA im BMA-Block. 


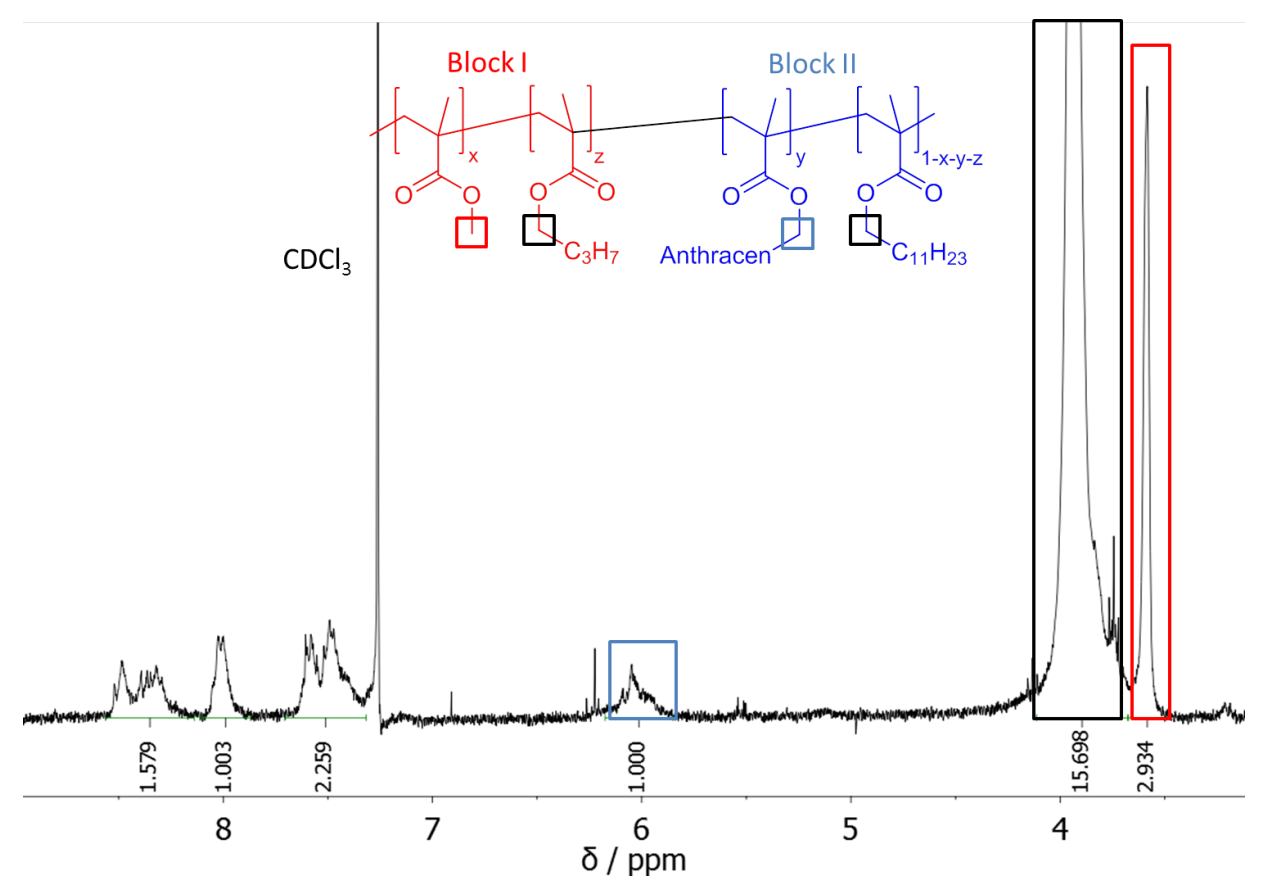

Abb. 5.8. ${ }^{1} \mathrm{H}-\mathrm{NMR}-\mathrm{Spektrum}$ von $\mathrm{P}(\mathrm{BMA}-\mathrm{co}-\mathrm{MMA})_{10 \mathrm{k}} \mathrm{P}(\mathrm{LMA}-\mathrm{co}-\mathrm{ANMA})_{19 \mathrm{k}}$. Für die Bestimmung der Zusammensetzung wurden die markierte Signale und die Veränderung des Verhältnisses des schwarz markierten zum rot markierten Signal vom Makro-RAFT-Agens gegenüber dem BCP verwendet.

\subsubsection{Untersuchung der Blockcopolymere mittels Rasterkraftmikroskopie}

\section{Bestimmung der Morphologie der MPS der ANMA enthaltenden Blockcopolymere}

Wie bereits erwähnt, ist die Ausbildung einer Mikrophasenseparation essentiell für dieses Projekt. Daher wurden analog zu den CMA-haltigen BCP im Abschnitt 5.2.3 dünne Filme der ANMA-haltigen BCP mittels AFM untersucht. Alle gezeigten Aufnahmen wurden mit einer Auflösung von $512 \times 512$ Pixel aufgenommen.

Abbildung 5.9 A zeigt das Höhenbild der Probe P(LMA-co-ANMA $\left.)_{49 k}\right)-P$ BMA $_{21 k}$ mit der höchsten Molmasse, die den Photovernetzer im weichen Block enthält.. Die Probe ist mikrophasensepariert, jedoch ist eine strikte Zuordnung der Morphologie nur schwer möglich. Die Linien können durch liegende Zylinder oder stehende Lamellen erzeugt werden. Die annähernd runden Strukturen sind vermutlich stehende Zylinder oder Sphären. Daher liegt vermutlich eine gemischte Morphologie aus liegenden und stehenden Zylindern, aus stehenden Lamellen und stehenden Zylindern oder aus liegenden Zylindern und Sphären vor. Ob stehende oder liegende Lamellen bzw. Zylinder entstehen, ist im wesentlichen von den Oberflächenspannungen der beiden Blöcke und der Substratoberfläche sowie der Wechselwirkungen an der Film-Luft-Grenzfläche abhängig. [209-211] Der Domänenabstand der Zylinder bzw. Lamellen ist wesentlich größer als der der Proben $\mathrm{P}(\mathrm{BMA}-\mathrm{co}-\mathrm{MMA})_{10 \mathrm{k}} \mathrm{P}(\mathrm{LMA}-$ co-ANMA $)_{19 k}$ und PLMA $21 k-P(B M A-c o-A N M A)_{21 k}$ in Abbildung 5.10, Dies könnte auf die Anwesenheit von Homopolymer des ersten oder zweiten Blockes zurückzuführen sein, wel- 
ches zur Quellung der Phasen führt. P(LMA-co-ANMA) $\left.{ }_{52 k}\right)-P_{B M A}{ }_{13 k}$ besitzt eine höhere Molmasse und eine höhere Dispersität von 1,4 als die anderen Polymere, da es vermutlich mehr Homopolymer aufgrund vermehrter Terminierungsreaktionen enthält.

In Abbildung 5.9 B wurde der selbe Ausschnitt unter Verwendung des PeakForceQuantitative Nanomechanical Mapping-Modus (PFQNM) aufgenommen. Mit diesem Modus werden an jedem Datenpunkt die mechanischen Eigenschaften, im speziellen der DMT(Derjaguin-Müller-Toporov)-Modul, der Oberfläche gemessen. 2121213 Besitzen die beiden Blöcke unterschiedliche Steifigkeiten so können die Phasen in den AFM-Aufnahmen den einzelnen Blöcken zugeordnet werden. ${ }^{[199 \mid 214]}$ Es stellte sich heraus, dass die DMT-ModulBestimmung mittels des verwendeten AFM nicht möglich ist. Trotz Kalibrierung nach Herstellerangaben entstehen zufällige und nicht sinnvolle DMT-Modulwerte. Daher wird der DMTModul in einer willkürlichen (engl. arbitrary, Arb) Einheit angegeben. Anhand des Vergleichs der Aufnahme des DMT-Oberflächenprofils und des Höhenprofils aus Abb. 5.9 kann festgestellt werden, dass die niedrigere Phase (dunklerer Bereich) im Höhenbild dem weicheren LMA-Block entspricht. Diese Zuordnung des weichen und harten Blockes ist bei den nachfolgenden Höhenprofilaufnahmen der anderen Blockcopolymere identisch und wurde hier aufgrund des größeren Domänenabstandes beispielhaft anhand der Probe P(LMA-coANMA) $)_{52 k}$ )-PBMA $13 k$ gezeigt.

Abbildung 5.10 A zeigt die topographische AFM-Aufnahmen der Probe P(BMA-co$M M A)_{10 k}-P\left(\right.$ LMA-Co-ANMA) ${ }_{19 k}$. Es wird eine Oberfläche mit einer Fingerabdruck-ähnlichen Struktur erhalten, die der aus Abschnitt 5.2.3 gleicht. Hierbei handelt es sich entweder um eine zylindrische Morphologie mit liegenden Zylindern oder um eine lamellare Morphologie mit stehenden Lamellen.

Die Probe $P L M A_{21 k}-P(B M A-c o-A N M A)_{11 k}$ hat eine ähnliche Molmasse und ein ähnliches Verhältnis des harten zum weichen Block wie $\mathrm{P}(\mathrm{BMA}-\text { co-MMA })_{10 \mathrm{k}}-\mathrm{P}(\text { LMA-co-ANMA })_{19 \mathrm{k}}$ (Abb. 5.10 A). Trotzdem zeigt Abbildung $5.10 \mathrm{~B}$, dass in dieser Probe die Blöcke nicht phasensepariert sind. Der fehlende Anteil an MMA im harten Block und der Einbau des
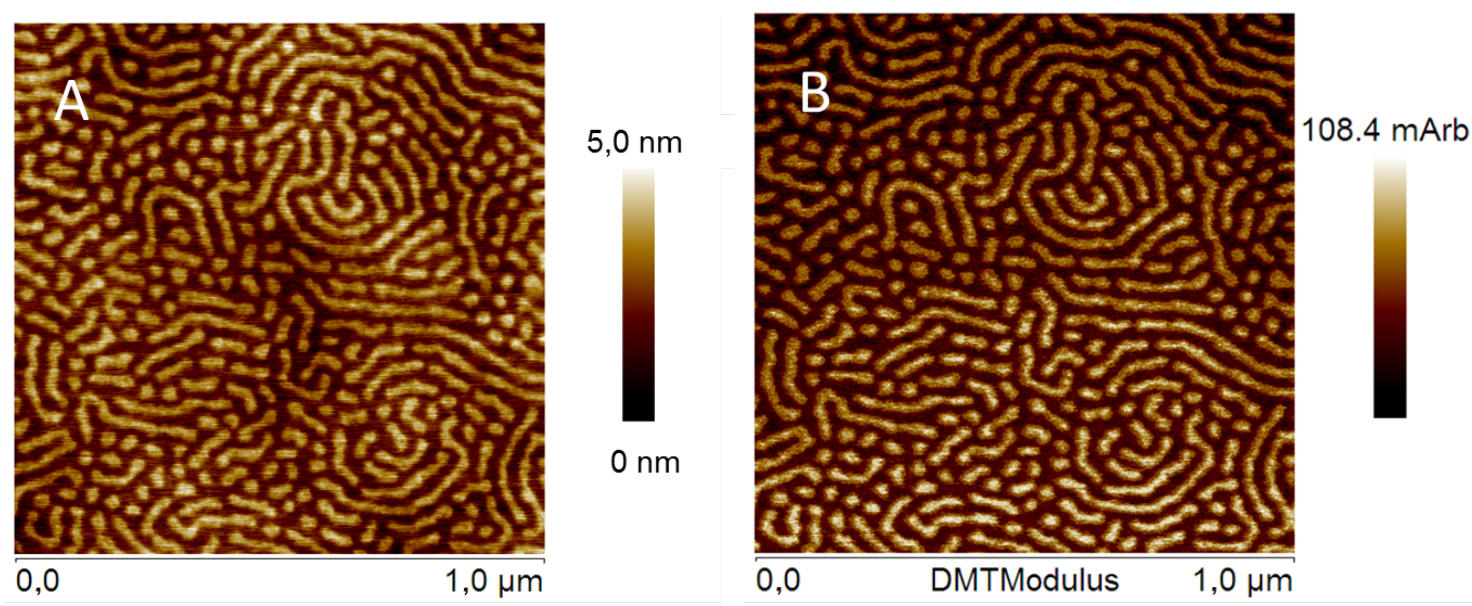

Abb. 5.9. Mittels AFM aufgenommenes Höhen- (A) und DMT-Modulprofile (B) der Oberfläche des Blockcopolymers $\left.\mathrm{P}(\mathrm{LMA}-\mathrm{co}-\mathrm{ANMA})_{52 \mathrm{k}}\right)-\mathrm{PBMA}_{13 \mathrm{k}}$. 

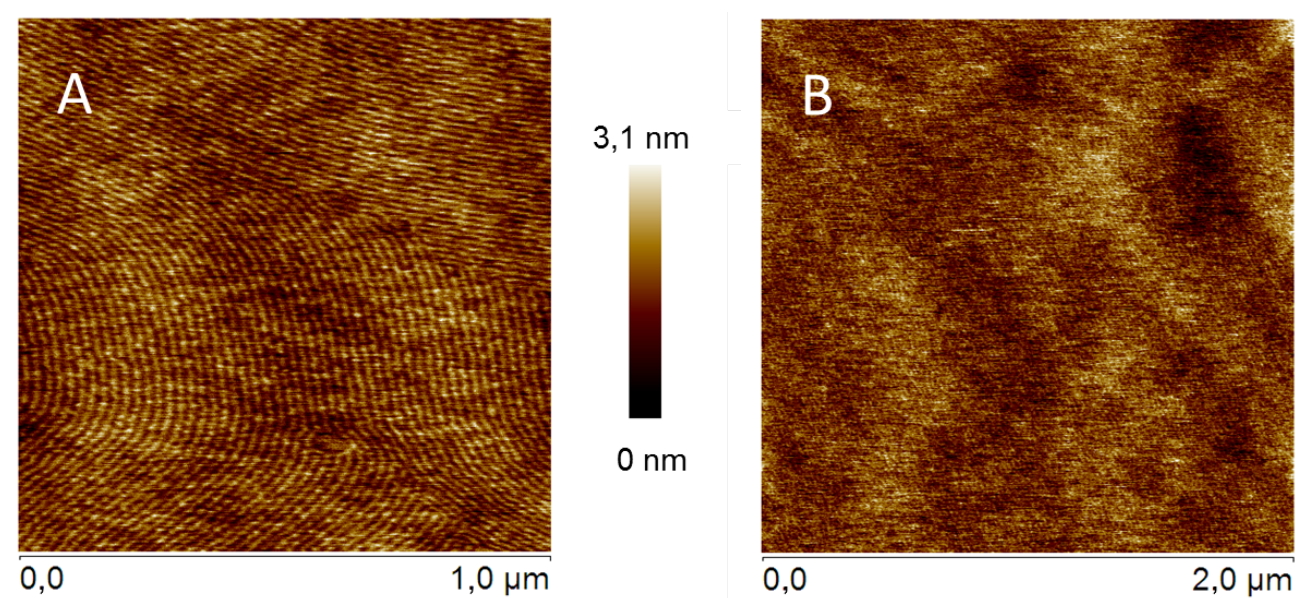

$2,7 \mathrm{~nm}$

0,0

$1,0 \mu \mathrm{m}$

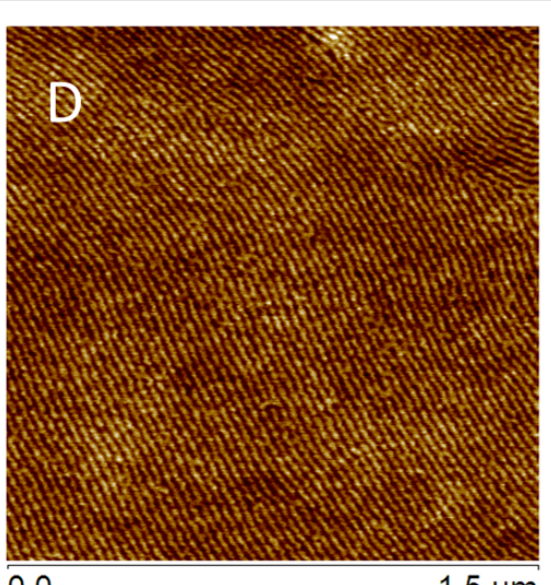

$6,0 \mathrm{~nm}$

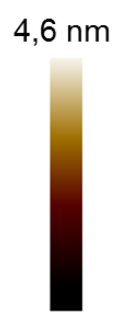

$0 \mathrm{~nm}$

0,0

$1,5 \mu \mathrm{m}$

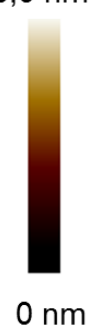

Abb. 5.10. Topographische AFM-Aufnahmen der Proben $P(B M A-c o-M M A)_{10 k}-P(L M A-c o-$ ANMA) $)_{19 k}$ (A), PLMA ${ }_{21 k}-P(B M A-c o-A N M A)_{11 k}$ (B), PLMA $2_{21 k}-P(B M A-c o-A N M A)_{17 k} \quad(C)$ und $\mathrm{PLMA}_{21 \mathrm{k}}-\mathrm{P}(\mathrm{BMA}-\mathrm{CO}-\mathrm{ANMA})_{21 \mathrm{k}}(\mathrm{D})$.

ANMA im harten statt weichen Block bei PLMA $21 \mathrm{k}-\mathrm{P}(\mathrm{BMA}-\mathrm{co}-\mathrm{ANMA})_{11 \mathrm{k}}$ hat demzufolge eine großen Einfluss auf den $\chi$-Parameter des BCP, sodass beide Blöcke bei gleichem Polymerisationsgrad mischbar sind. Daher wurde sukzessive die Molmasse des harten Blockes durch weitere Polymerisation erhöht. Steigt die Molmasse des BMA-Blocks auf $17 \mathrm{~kg} \mathrm{~mol}^{-1}$, so entsteht eine zylindrische Morphologie (Abb. 5.10 C). Jedoch befindet sich das System in der weak segregation region bzw. kurz oberhalb dieser. Die Blöcke beginnen sich zu entmischen, aber es entsteht noch keine geordnete Struktur. Nach weitere Verlängerung des BMA-Blocks auf $21 \mathrm{~kg} \mathrm{~mol}^{-1}$ entsteht eine geordnete zylindrische Morphologie (Abb. 5.10 D), die analog zu der der Probe $P(B M A-c o-M M A)_{10 k}-P(\text { LMA-co-ANMA })_{19 k}($ Abb. 5.10 A) ist.

Bei $P(\text { LMA-co-ANMA) })_{21 k}-P(B M A-c o-A N M A)_{10 k}$ wurde ebenfalls keine MPS erhalten (Abb. A9 A im Anhang), obwohl die Molmasse und das Verhältnis des harten zum weichen Block ist ähnlich zu der der Probe P(BMA-co-MMA) ${ }_{10 k}-\mathrm{P}\left(\right.$ LMA-co-ANMA) ${ }_{19 k}($ Abb. $5.10 \mathrm{~A})$. Der fehlende Anteil an MMA im harten Block und der Einbau des ANMA in beiden Blöcken hat auch hier einen großen Einfluss auf den $\chi$-Parameter des BCP. Auch durch Verlängerung 
des BMA-Blockes auf $27 \mathrm{~kg} \mathrm{~mol}^{-1}$ konnte keine MPS erhalten werden (Abb. A9 B).

\section{Untersuchung des Ordnungs-Unordnungs-Übergangs}

Nachdem bei dem Cumarin-BCP-System im Abschnitt 5.2.4 der ODT erst bei über $190{ }^{\circ} \mathrm{C}$ stattfindet, wurde der ODT auch in den Anthracen-BCP-Systemen anhand der Probe $\mathrm{P}$ (BMA-co-MMA) ${ }_{10 \mathrm{k}}-\mathrm{P}(\mathrm{LMA}-\mathrm{co}-\mathrm{ANMA})_{19 \mathrm{k}}$ untersucht.

Abbildungen 5.11 $A$ und $B$ zeigen die AFM-Aufnahmen dieses Blockcopolymers nach Tempern bei $120^{\circ} \mathrm{C}$ (A) bzw. $150{ }^{\circ} \mathrm{C}$ (B) und schnellen Abkühlen auf Raumtemperatur. Bei $120^{\circ} \mathrm{C}$ tritt die bereits im vorherigen Abschnitt beschriebene geordnete Struktur auf, was durch das zweite Maximum der spectral density Fourier Transformation im Innenbild bestätigt wird. Nach Tempern bei $150{ }^{\circ} \mathrm{C}$ geht die geordnete Struktur verloren, was auch anhand des fehlenden zweiten Maximums in der spectral density Fourier Transformation verdeutlicht wird. Nach erneutem Tempern bei $120^{\circ} \mathrm{C}$ sollte sich wieder die MPS ausbilden.

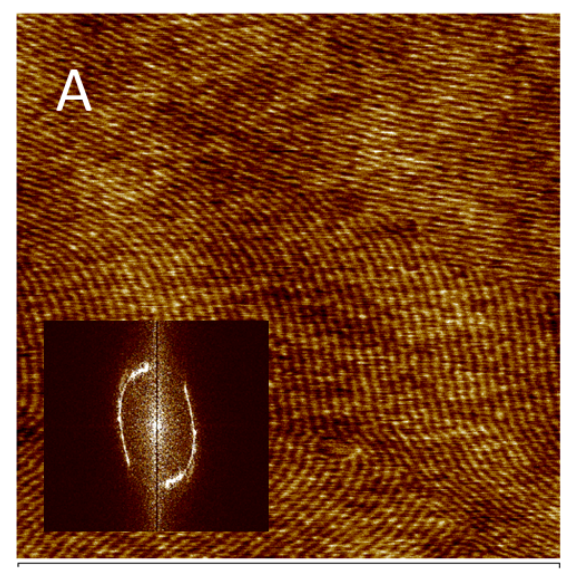

0,0

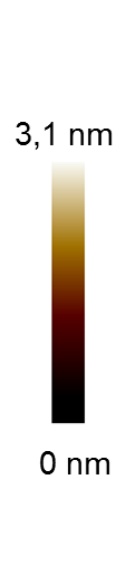

$1,0 \mu \mathrm{m}$
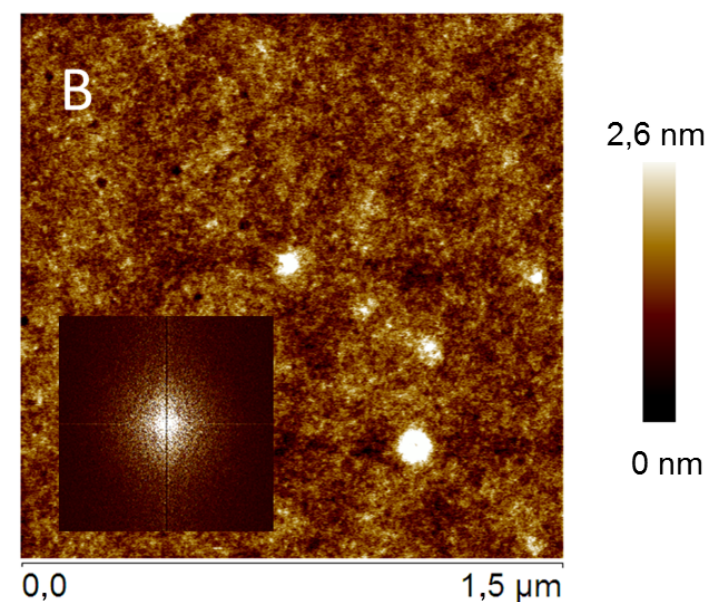

$0 \mathrm{~nm}$

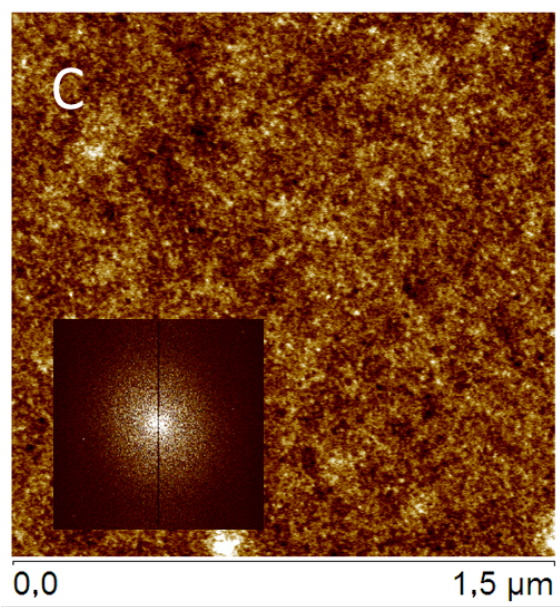

$2,7 \mathrm{~nm}$

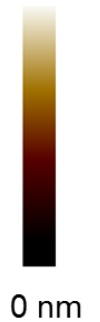

Abb. 5.11. Topographische AFM-Aufnahmen des BCP $P(B M A-c o-M M A)_{10 k}-P(L M A-c o-$ ANMA) ${ }_{19 k}$ nach Tempern bei $120^{\circ} \mathrm{C}(\mathrm{A})$ und $150^{\circ} \mathrm{C}(\mathrm{B})$ sowie $120^{\circ} \mathrm{C}$ nach vorherigem Tempern bei $150^{\circ} \mathrm{C}$. Alle Messungen fanden bei Raumtemperatur statt. Die Innenbilder zeigen die spectral density Fourier Transformation der jeweiligen Höhenbilder. 
Abbildung 5.11 zeigt jedoch, dass sich die MPS nicht wiederherstellt. Der ODT ist auch hier nicht reversibel.

Sowohl in dem Cumarin-BCP als auch in dem Anthracen-BCP konnte kein reversibler ODT gefunden werden. Möglich ist, dass oberhalb des ODT bei $150^{\circ} \mathrm{C}$ bzw. $195{ }^{\circ} \mathrm{C} \mathrm{Ne}$ benreaktionen stattfinden, die die Polymerstruktur verändern. Eine andere Erklärung könnte sein, dass AFM nicht die geeignete Untersuchungsmethode ist, da nur die oberste Polymerschicht untersucht wird und diese vor allem Anfällig für Beschädigungen und Nebenreaktion ist.

\subsubsection{Untersuchung der reversiblen Photovernetzung in dünnen Filmen}

Die reversible Photovernetzung wird auch bei den Anthracen-BCP mittels UV/VISSpektroskopie untersucht. Dabei wird analog zu der bereits in Abschnitt 5.2.5 beschriebenen Vorgehensweise verfahren. Die photochemische Vernetzung wurde in einem Glaskolben unter Argonatmosphäre durchgeführt, um die Photooxidation der Anthracengruppen zu vermeiden. 215-217] Ebenso wurden die Proben zur Entnetzung unter Argonatmosphäre thermisch behandelt.

Abbildung 5.12 A zeigt ein UV/VIS-Spektrum eines dünnen Films der Probe $\mathrm{P}\left(\mathrm{LMA}_{0,95}-\mathrm{CO}\right.$ $\mathrm{ANMA}_{0,05}$ ) mit 5 mol\% ANMA. Während der Photovernetzung nimmt die Absorption des Anthracenchromophore ab, da das Anthracendimer nach der Vernetzung nicht mehr im untersuchten Wellenlängenbereich absorbiert. Die Probe wurde insgesamt 13 Minuten bestrahlt. Die Photovernetzung ist wesentlich schneller als im Falle des Cumarin-Polymers (Abbildung 5.5), da bei $\mathrm{P}\left(\mathrm{LMA}_{0,95}-\mathrm{Co}-\mathrm{ANMA}_{0,05}\right)$ das ANMA in einem Polymer mit einem $T_{\mathrm{g}}$ unterhalb der Raumtemperatur eingebaut wurde. Da normales Glas statt Quarzglas verwendet wurde, ist im Spektrum der Wellenlängenbereich aufgrund Rauschens durch die Absorption des Glasplättchens unterhalb von $325 \mathrm{~nm}$ abgeschnitten. Abbildung 5.12 B zeigt die Entwick-
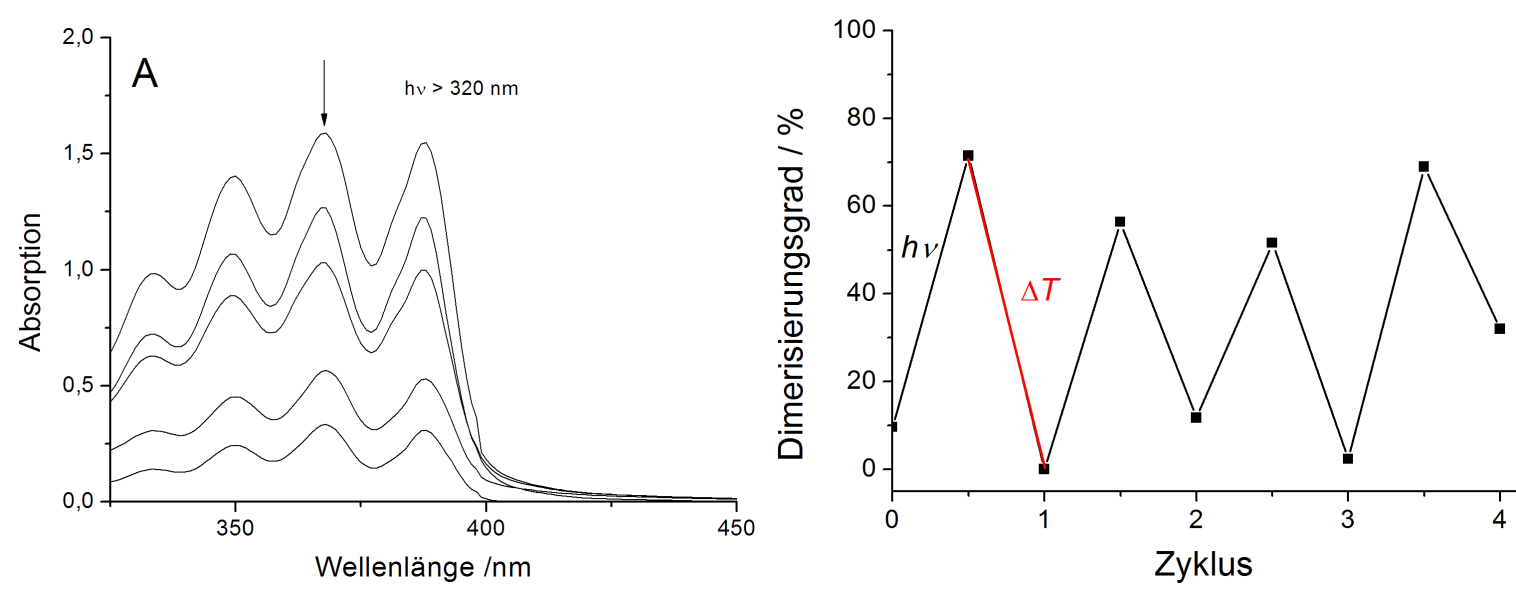

Abb. 5.12. A: UV/VIS-Spektrum des Anthracen enthaltenden Polymers $P\left(L_{M A} A_{0,95}-\mathrm{CO}-\right.$ $\left.\mathrm{ANMA}_{0,05}\right)$. Der schwarze Pfeil gibt die Entwicklung der Bande während der Bestrahlung mit $h \nu>320 \mathrm{~nm}$ an. B: Entwicklung des Dimerisierungsgrades, bestimmt anhand der normierten Absorption bei $367 \mathrm{~nm}$, über mehrere Zyklen der Ver- und Entnetzung. 
lung des Dimeriersierungsgrades der Anthracengruppen über mehrere Zyklen der Ver- und Entnetzung. Der Dimerisierungsgrad wurde anhand der normierten Absorption am Absorptionsmaximum bestimmt. Durch die Photovernetzung steigt der Dimerisierungsgrad an und nach der thermischen Behandlung der Probe nimmt er wieder ab. Der Dimerisierungsgrad des einmal thermisch entnetzten Films (Zyklus 1) ist geringer als der des unbestrahlten und unbehandelten Films. Dies könnte darauf zurückzuführen sein, dass die unbehandelte Probe während der Filmherstellung nicht vollständig vor Tageslicht geschützt war, sodass ein Teil der Anthracengruppen bereits dimerisierte. Die Reversibilität der Photovernetzung ist für mindestens 3 Zyklen nahezu quantitativ. Insbesondere die Entnetzung ist dabei wesentlich effektiver als die des in Abschnitt5.2.5 untersuchten Cumarinpolymers.

\subsubsection{Bestimmung der Glasübergangstemperaturen}

Für die Bestimmung der mechanischen Eigenschaften der unvernetzten und vernetzten ANMA-Copolymere werden Zugversuche bei Raumtemperatur durchgeführt. Da die mechanischen Eigenschaften unter anderem durch den Glasübergang stark von der Temperatur abhängig sind, ist es von großer Bedeutung die Glasübergangstemperatur(en) der Polymere zu kennen.

Eine Methode zur Bestimmung des Glasübergangstemperaturen ist die dynamischmechanischen Analyse (DMA). ${ }^{218}$ Während der dynamisch-mechanischen Analyse wird die Probe einer sinusförmigen Belastung in Abhängigkeit der Temperatur ausgesetzt. In dieser Arbeit wurde für die periodische Belastung der 1-Punkt-Biegeversuch verwendet. Im Bereich der Glasübergangstemperatur erweicht das Polymer und der Biegemodul $B$ nimmt sprunghaft ab. Der elastische Part des Biegemoduls ist der Speichermodul $B^{\prime}$, dessen Wert ebenfalls sprunghaft im Bereich des Glasüberganges abnimmt. Der Verlustmodul $B^{\prime \prime}$ spiegelt den viskosen Anteil am Biegemodul wieder. Der Verlustfaktor $\tan (\delta)$ entspricht nach Gleichung 5.1 dem Quotienten aus Verlust- und Speichermodul. Anhand des Minimums in der Ableitung der Speichermodulkurve, des Maximums des Verlustmoduls und des Maximums des Verlustfaktors kann die Glasübergangstemperatur bestimmt werden. Die mittels $B^{\prime}, B^{\prime \prime}$ und $\tan (\delta)$ ermittelten $T_{\mathrm{g}}$ variieren leicht.

$$
\tan (\delta)=\frac{B^{\prime \prime}}{B^{\prime}}
$$

\section{Ermittlung der Glasübergangstemperaturen der statistischen Copolymere}

Die Glasübergangstemperaturen werden im Folgenden mittels dynamisch-mechanischer Analyse ermittelt (DMA), da eine Bestimmung der $T_{\mathrm{g}}$ mittels dynamische Differenzkalorimetrie (DSC) nicht möglich war. Das könnte daran liegen, dass das Homopolymer PLMA teilkristallin ist und die Sensitvität des DSC daher nicht ausreicht. ${ }^{[19220]}$ Die DMA ist jedoch sensitiver als die DSC in der Bestimmung des $T_{\mathrm{g}}$. 2200 Die meisten unvernetzten statistischen Copolymere aus Tabelle 5.3 fließen bei Raumtemperatur, sodass keine Probenkörper hergestellt werden können. Daher wurden die Proben mit Hilfe einer Materialtasche 
gemessen. Dazu werden 20 bis $40 \mathrm{mg}$ der Probe in die Taschen aus Stahl gegeben und die Tasche zugebogen. Die Tasche wurde dann ebenfalls im 1-Punkt-Biegeversuch vermessen. Dabei wird hauptsächlich der Modul der Tasche gemessen, diese sind für die Probe nicht repräsentativ. Im Bereich des Glasübergangs des Polymers fällt $B^{\prime}$ aufgrund des sich erweichenden Polymers innerhalb der Materialtasche trotzdem ab bzw. $B^{\prime \prime}$ und $\tan (\delta)$ zeigen trotzdem ein Maximum. Die mit Hilfe der Materialtaschen bestimmten Glasübergangstemperaturen sind identisch mit den $T_{\mathrm{g}}$, der anhand eines Probenkörpers bestimmt wurde. 221]

Abbildung 5.13 zeigt anhand $\mathrm{P}\left(\mathrm{LMA}_{0,95}-\mathrm{Co}-\mathrm{ANMA}_{0,05}\right)$ beispielhaft die mittels der Materialtaschen in der DMA erhaltenen temperaturabhängigen Kurven des Speichermoduls, Verlustmoduls und des Verlustfaktors. Eine Bestimmung des $T_{\mathrm{g}}$ ist sowohl anhand des Maximums des $\tan (\delta)$ und des $B^{\prime \prime}$ als auch anhand des Minimums der Ableitungskurve des Speichermodul möglich. In dieser Arbeit wird bei allen Polymere die Glasübergangstemperatur bevorzugt anhand des Maximums des $\tan (\delta)$ bestimmt.

Tabelle 5.7 zeit die anhand des $\tan (\delta)$ ermittelten Glasübergangstemperaturen der vier statistischen Copolymere. Es ist bekannt, dass in statistischen Copolymeren der $T_{\mathrm{g}}$ zwischen dem $T_{\mathrm{g}}$ beider Homopolymere liegt. 222[223] Alle Copolymere aus LMA und ANMA weisen eine höhere Glasübergangstemperatur auf als der in der Literatur bestimmte $T_{\mathrm{g}}$ von

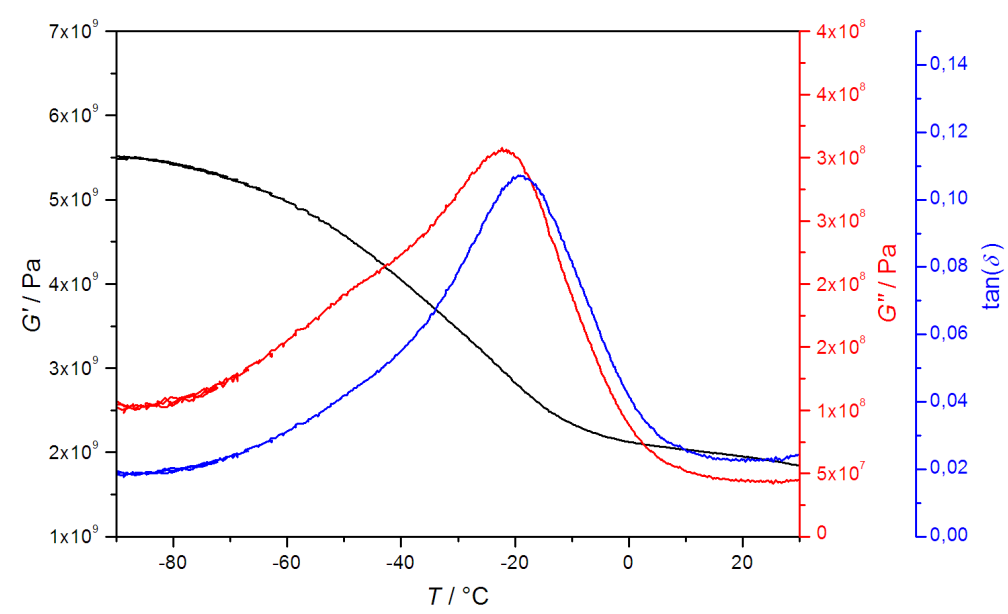

Abb. 5.13. Mittels Materialtaschen erhaltene DMA-Kurven der Probe $P\left(L M A_{0,95}-c o-A N M A_{0,05}\right)$.

Tab. 5.7. Die Zusammensetzungen der statistischen Polymere sowie deren Glasübergangstemperaturen, die mittels DMA anhand des Maximums des Verlustfaktors bestimmt wurden.

\begin{tabular}{|c|c|c|}
\hline Probe & $\left.\mathrm{F}_{\text {ANMA }}\right|^{\sqrt{a}} / \%$ & $T_{\mathrm{g}} /{ }^{\circ} \mathrm{C}$ \\
\hline $\mathrm{P}\left(\mathrm{LMA}_{0,85}-\mathrm{co}-\mathrm{ANMA}_{0,15}\right)$ & 15 & 0 \\
\hline $\mathrm{P}\left(\mathrm{LMA}_{0,92}-\right.$ co-ANMA $\left.\mathrm{A}_{0,08}\right)$ & 8 & -12 \\
\hline $\mathrm{P}\left(\mathrm{LMA}_{0,95}-\mathrm{Co}^{\left.-\mathrm{ANMA}_{0,05}\right)}\right.$ & 5 & -19 \\
\hline $\mathrm{P}\left(\mathrm{BMA}_{0,9}-\mathrm{Co}-\mathrm{ANMA}_{0,1}\right)$ & 10 & 60 \\
\hline
\end{tabular}

${ }^{a}$ Anteil ANMA im Polymer bestimmt über ${ }^{1} \mathrm{H}$-NMR-Spektroskopie. 
-48 bis $-65^{\circ} \mathrm{C}$ für das Homopolymer PLMA. 2051206] Zudem steigt die Glasübergangstemperatur mit zunehmenden Anteil des ANMA an. Daraus lässt sich schließen, dass der Glasübergang des ANMA-Homopolymers bei höheren Temperaturen liegt, als der des PLAM. Obwohl der $T_{\mathrm{g}}$ des ANMA-Homopolymers nicht gemessen wurde bzw. nicht bekannt ist, ist diese Beobachtung mit der sterisch anspruchsvolleren und weniger flexiblen Seitenkette des ANMA zu erklären, die den $T_{\mathrm{g}}$ des Copolymers gegenüber dem Homopolymer PLMA erhöht. ${ }^{119223]}$ Der $T_{\mathrm{g}}$ des Copolymers $\mathrm{P}\left(\mathrm{BMA}_{0,9}-\mathrm{Co}-\mathrm{ANMA}_{0,1}\right)$ ist mit $60^{\circ} \mathrm{C}$ etwas höher als der Literaturwert von etwa $53^{\circ} \mathrm{C}$ für das Homopolymer PBMA, der ebenfalls über den $\tan (\delta)$ bestimmt wurde. ${ }^{208}$ Daher ist anzunehmen, dass das Homopolymer PANMA aufgrund seiner sterisch anspruchsvollen Struktur und geringeren Flexibilität einen höheren $T_{\mathrm{g}}$ als PBMA hat.

\section{Ermittlung der Glasübergangstemperaturen der Blockcopolymere}

Die Blockcopolymere $\mathrm{P}(\mathrm{BMA}-\mathrm{co}-\mathrm{MMA})_{10 k}-\mathrm{P}(\mathrm{LMA}-\mathrm{co}-\mathrm{ANMA})_{19 k}$ und $\mathrm{PLMA}_{21 \mathrm{k}}-\mathrm{P}(\mathrm{BMA}-\mathrm{co}-$ ANMA) $)_{21 k}$ werden zur Untersuchung des Einflusses der Photovernetzung auf deren mechanischen Eigenschaften verwendet. Daher wurde auch die Glasübergangstemperaturen dieser beiden Polymere bestimmt.

Die Abbildungen 5.14 A und B zeigen die mittels der Materialtaschen in der DMA erhaltenen temperaturabhängigen Kurven des Speichermoduls, Verlustmoduls und des Verlustfaktors der Blockcopolymere P(BMA-co-MMA) ${ }_{10 k^{-}} \mathrm{P}(\mathrm{LMA}-\mathrm{co}-\mathrm{ANMA})_{19 \mathrm{k}}$ (A) und PLMA $\mathrm{PLk}_{21 \mathrm{~K}^{-}}$ $\mathrm{P}(\mathrm{BMA}-\mathrm{co}-\mathrm{ANMA})_{21 \mathrm{k}}$ (B). Blockcopolymere, die Mikrophasenseparation zeigen, besitzen für beide Blöcke jeweils einen Glasübergang. ${ }^{[224]}$ Die Glasübergangstemperatur des weichen Blockes ist bei beiden Polymeren deutlich anhand des $\tan (\delta)$ zu erkennen und liegt bei $-3{ }^{\circ} \mathrm{C}$ für $\mathrm{P}(\mathrm{BMA}-\mathrm{co}-\mathrm{MMA})_{10 \mathrm{k}}-\mathrm{P}(\mathrm{LMA}-\mathrm{co}-\mathrm{ANMA})_{19 \mathrm{k}}$ und $-13^{\circ} \mathrm{C}$ für $\mathrm{PLMA} \mathrm{Ak}_{21 \mathrm{k}}-\mathrm{P}$ (BMA-coANMA) $21 \mathrm{k}$. Der $T_{\mathrm{g}}$ ist bei ersterem durch den Anteil von $5 \mathrm{~mol} \%$ ANMA etwas höher. Auch im Vergleich zum statistischen Copolymer $\mathrm{P}\left(\mathrm{LMA}_{0,95}-\mathrm{CO}-\mathrm{ANMA}_{0,05}\right)$, welches ebenfalls etwa 5 mol\% ANMA enthält, ist der $T_{\mathrm{g}} \mathrm{P}(\mathrm{BMA}-\mathrm{Co}-\mathrm{MMA})_{10 \mathrm{k}} \mathrm{P}(\mathrm{LMA}-\mathrm{Co}-\mathrm{ANMA})_{19 \mathrm{k}}$ etwa $15^{\circ} \mathrm{C}$ höher. Bei Blockcopolymeren verschieben sich die $T_{\mathrm{g}}$ beider Blöcke aufeinander zu, da ein Teil der Kette des einen Blockes in die jeweils andere Phase übergehen kann. ${ }^{2244}$ Der harte Block erhöht also den $T_{\mathrm{g}}$ des weichen Blockes.

Bei $\mathrm{P}(\mathrm{BMA}-\mathrm{co}-\mathrm{MMA})_{10 \mathrm{k}}-\mathrm{P}(\mathrm{LMA}-\mathrm{co}-\mathrm{ANMA})_{19 \mathrm{k}}$ konnte weder mit den Materialtaschen noch mit der Messung von Probenkörper ein $T_{\mathrm{g}}$ des harten Blockes ermittelt werden, obwohl die Probe Mikrophasenseparation zeigt (siehe Abschnitt 5.3.3). Ursache dafür ist vermutlich, dass sich bei Überschreiten des ersten $T_{\mathrm{g}}$ die Feststellschrauben in der DMA lösen und die Probe dann nicht mehr fest eingespannt ist. Bei PLMA $21 \mathrm{~K}^{-}$ $\mathrm{P}(\mathrm{BMA}-\mathrm{co}-\mathrm{ANMA})_{21 \mathrm{k}}$ konnte anhand des Wendepunktes des Speichermoduls ein $T_{\mathrm{g}}$ von $58{ }^{\circ} \mathrm{C}$ für den BMA-Block ermittelt werden. Der $\tan (\delta)$ zeigt jedoch nur für den LMA-Block einen $T_{\mathrm{g}}$. Beide BCP wurden daher auch mittels DSC analysiert, welche den Wärmefluss in Abhängigkeit der Temperatur misst. Durch die DSC konnten die Glasübergangstemperaturen der BMA-Blöcke beider BCP ermittelt werden. Dabei ist der $T_{\mathrm{g}}$ bei $\mathrm{P}(\mathrm{BMA}-\mathrm{co}-\mathrm{MMA})_{10 \mathrm{k}}-\mathrm{P}(\mathrm{LMA}-\mathrm{co}-\mathrm{ANMA})_{19 \mathrm{k}}$ vermutlich aufgrund der kürzeren 

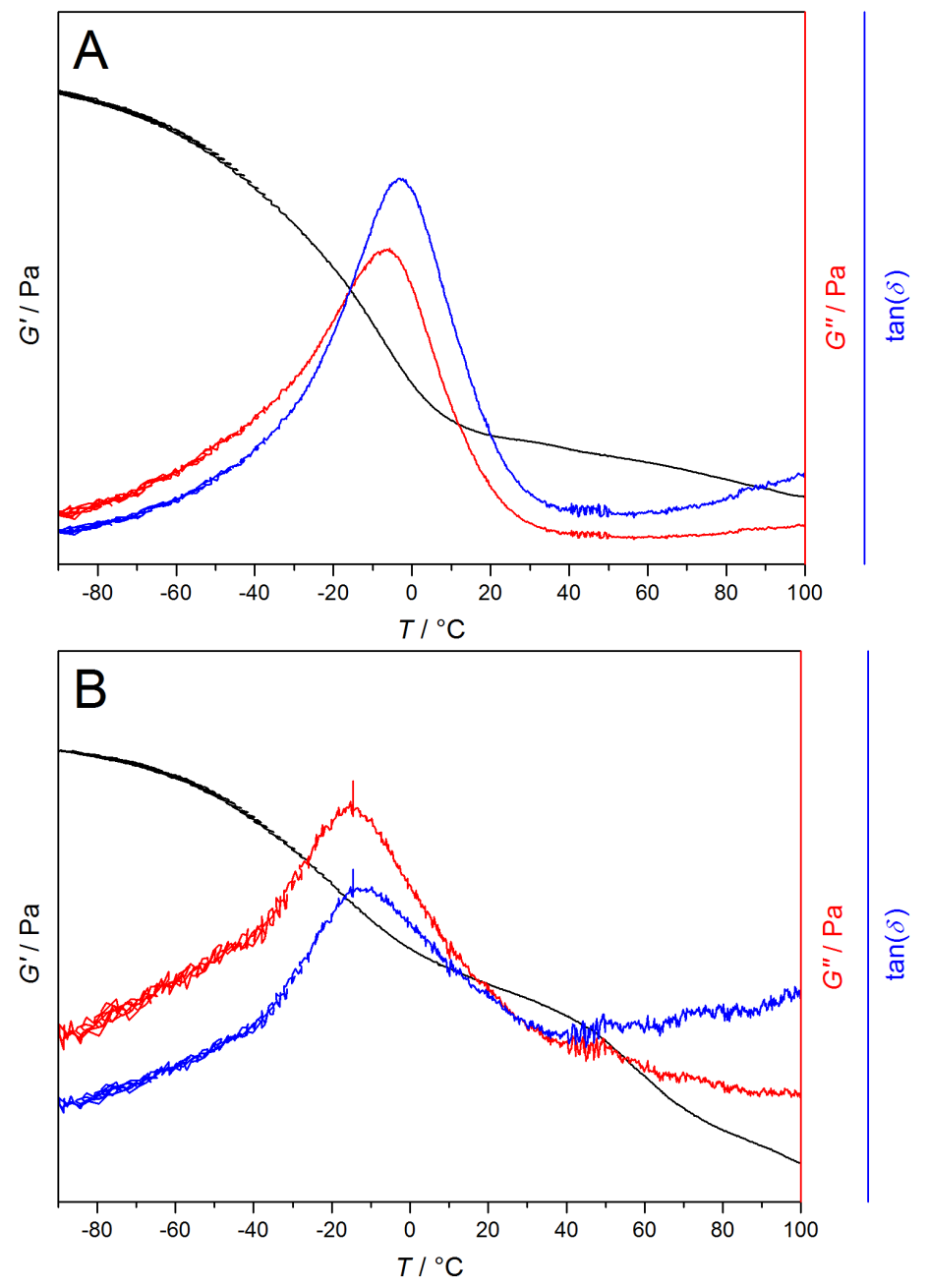

Abb. 5.14. DMA-Kurven der Blockcopolymere $P(B M A-c o-M M A)_{10 k}-P(L M A-c o-A N M A)_{19 k}(A)$ und $\mathrm{PLMA}_{21 \mathrm{k}}-\mathrm{P}(\mathrm{BMA}-\mathrm{co}-\mathrm{ANMA})_{21 \mathrm{k}}(\mathrm{B})$. Die Absolutwerte spiegeln hauptsächlich den Speicher- und Verlustmodul der Materialtasche wieder.

Tab. 5.8. Die mittels DMA und DSC ermittelten Glasübergangstemperaturen der Blockcopolymere.

\begin{tabular}{|c|c|c|c|}
\hline \multirow[t]{2}{*}{ Polymer } & \multicolumn{2}{|c|}{ DMA } & \multirow{2}{*}{$\begin{array}{c}\text { DSC } \\
T_{\mathrm{g}, \mathrm{BMA}}\end{array}$} \\
\hline & $T_{\mathrm{g}, \mathrm{LMA}}$ & $T_{\mathrm{g}, \mathrm{BMA}}$ & \\
\hline $\begin{array}{c}\mathrm{P}(\mathrm{BMA}-\mathrm{co}-\mathrm{MMA})_{10 \mathrm{k}}-\mathrm{P}(\mathrm{LMA}-\text { co-ANMA })_{19 \mathrm{k}} \\
\mathrm{PLMA}_{21 \mathrm{k}}-\mathrm{P}(\mathrm{BMA}-\text { co-ANMA })_{21 \mathrm{k}}\end{array}$ & $\begin{array}{l}-3^{a} \\
-13^{3}\end{array}$ & $588^{b}$ & $\begin{array}{l}39 \\
46\end{array}$ \\
\hline
\end{tabular}

${ }^{a}$ Bestimmt anhand des Maximums des Verlustfaktors.

${ }^{b}$ Bestimmung war mittels DMA nicht möglich.

${ }^{c}$ Bestimmung anhand des Wendepunktes der Speichermodulkurve. 
BMA-Blocklänge niedriger und das Signal des $T_{\mathrm{g}}$ weniger ausgeprägt (Abb. A10 im Anhang). Der $T_{\mathrm{g}}$ sinkt mit abnehmender Molmasse. 11112] Des Weiteren kann es auch sein, dass ANMA als Comonomer den $T_{\mathrm{g}}$ des BMA-Blockes stärker erhöht als das Comonomer MMA. Tabelle 5.8 fasst die mittels DMA und DSC ermittelten $T_{\mathrm{g}}$ der beiden Blockcopolymere zusammen. Der $T_{\mathrm{g}}$ des BMA-Blockes unterscheidet sich je nach Messmethode und ist in der DSC niedriger als in der DMA. Diese Beobachtung, dass der $T_{\mathrm{g}}$ abhängig von der verwendeten Messmethode und den Messparametern ist, ist literaturbekannt. 22252226]

\subsubsection{Untersuchung des Einflusses der Photovernetzung auf die mechanischen Eigenschaften der statistischen Copolymere}

Für die Bestimmung der mechanischen Eigenschaften anhand des Zugversuches wurden knochenförmige Probenkörper mit Hilfe von Negativformen aus Teflon hergestellt. Eine 20 bis 30 wt.\%-ige Lösung des Polymers in Dioxan wurde in die Form gegeben und das Lösungsmittel wurde durch langsame Druckerniedrigung bei $85^{\circ} \mathrm{C}$ entfernt. Nach mehrmaligem Auffüllen konnte homogene Probenkörper erhalten werden. Probenkörper des Polymers $\mathrm{P}\left(\mathrm{BMA}_{0,9}-\mathrm{Co}-\mathrm{ANMA}_{0,1}\right)$ konnten mittels dieses Verfahrens nicht blasenfrei hergestellt werden. Mit dem Spritzgussverfahren verfärbte sich diese Probe oder sie konnte nicht in einem Stück aus der Form genommen werden. Daher wurden die mechanischen Eigenschaften des $\mathrm{P}\left(\mathrm{BMA}_{0,9}-\mathrm{co}-\mathrm{ANMA}_{0,1}\right)$ nicht ermittelt. Jede Probe wurde zweimal gemessen und die Mittelwerte samt der Standardabweichung werden im Folgenden angegeben.

Probenkörper der Polymere $\mathrm{P}\left(\mathrm{LMA}_{0,85}-\mathrm{co}-\mathrm{ANMA}_{0,15}\right), \mathrm{P}\left(\mathrm{LMA}_{0,92}-\mathrm{co}-\mathrm{ANMA}_{0,08}\right)$ und $\mathrm{P}\left(\mathrm{LMA}_{0,95}-\mathrm{Co}-\mathrm{ANMA}_{0,05}\right)$ wurden von beiden Seiten für unterschiedliche Zeiten mittels einer $1500 \mathrm{~W}$-Quecksilberlampe in einer Kammer unter leichtem Argonstrom bestrahlt. Eine angegebene Bestrahlungsdauer von $2 \mathrm{~h}$ bedeutet, dass die Probe jeweils $2 \mathrm{~h}$ von beiden Seiten bestrahlt wurde. Während der Bestrahlung stieg die Temperatur der Bestrahlungskammer auf auf größer $80^{\circ} \mathrm{C}$, sodass die $T_{\mathrm{g}}$ aller Copolymere überschritten werden. Durch das Anlegen eines leichten Argonstroms wurde versucht eine Inertgasatmosphäre zu erzeugen jedoch stoppte der Argonstrom nach unbestimmter Zeit. Von der Verwendung eines höheren Argonstroms wurde abgesehen, da sich die Argonflasche innerhalb ein bis zwei Stunden leerte und so unverhältnismäßige Kosten entstanden. Es ist daher davon auszugehen, dass dauerhaft keine Inertgasatmosphäre in der Bestrahlungskammer erzeugt wurde. Diese Vermutung wird durch den ozonartigen Geruch, der während und nach dem Bestrahlen im Raum vorhanden war, gestützt. Mit zunehmender Bestrahlungszeit verdunkeln sich die Probenkörper wie in der Abbildung A11 im Anhang gezeigt. Diese Verfärbungen könnten ein Indiz für Nebenreaktionen sein. 

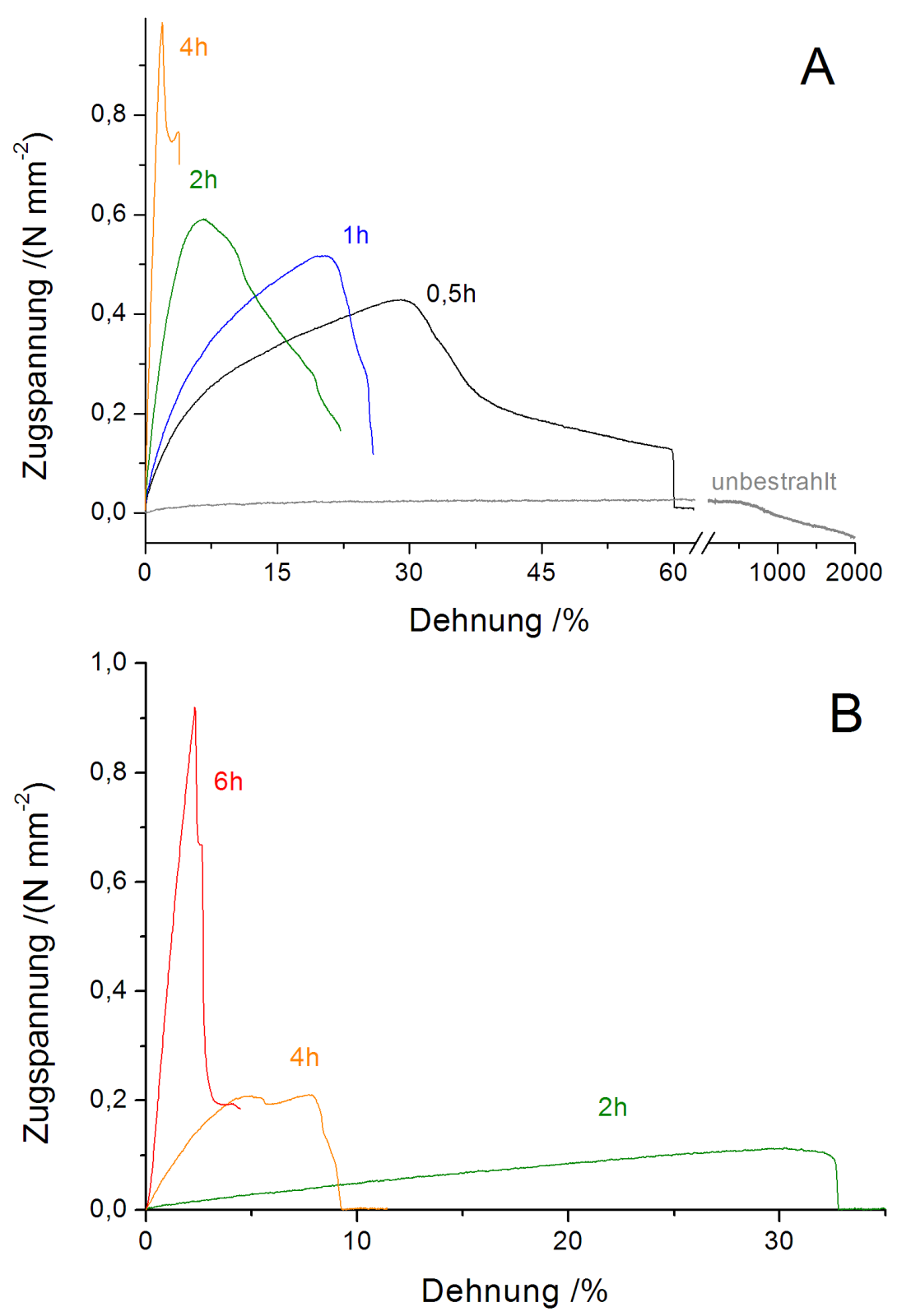

Abb. 5.15. Zugkurven der Proben $P\left(L_{M A} A_{0,85}-c o-A N M A_{0,15}\right)(A)$ und $P\left(L_{M A} A_{0,95}-c o-A N M A_{0,05}\right)(B)$ nach unterschiedlicher Bestrahlungsdauer.

Abbildung 5.15 A zeigt die Zugkurven der unterschiedlich lang bestrahlten Proben des statistischen LMA-ANMA-Copolymers mit dem höchsten Anteil von $15 \%$ an ANMA. Die unbestrahlte Probe ist sehr weich und reißt selbst nach einer Dehnung von $2000 \%$ nicht, da ihr $T_{\mathrm{g}}$ mit $0{ }^{\circ} \mathrm{C}$ unter der Raumtemperatur liegt und sie daher hochviskos ist. Die Polymerketten können aneinander vorbei fließen. Die genauen Werte Ergebnisse der Zugversuche finden sich in Tabelle 5.9 wieder. Nach vier Stunden Bestrahldauer erhöht sich der E-Modul um den Faktor 120 bis 130 und die Bruchdehnung $\epsilon_{\mathrm{B}}$ der Probe verkürzt sich rapide auf $5 \%$. Durch die Bestrahlung wird die Probe vernetzt, wodurch die Fließfähigkeit der Ketten 
reduziert wird. Zugleich sorgen die Vernetzungsstellen für mechanische Stabilität und erhöhen so den E-Modul. Mit der Bestrahlungsdauer erhöht sich aus dem gleichen Grund auch die Zugfestigkeit $R_{\mathrm{m}}$, also die maximale Zugspannung, die der Probekörper aushält. Die Photovernetzung auf Basis der [4+4] Cycloaddition ist demnach auch in bis zu 1,5 mm dicken Probenkörper erfolgreich. Durch die Bestrahlung können auch zwei Anthracengruppen innerhalb einer Polymerkette dimerisieren. Dies dürtte ebenfalls die Stabilität erhöhen sowie die Flexibilität und das Fließverhalten reduzieren. Die Zähigkeit, die Gesamtenergie die der Probenkörper aufnehmen kann, reduziert sich mit zunehmender Bestrahlungsdauer, da die Bruchdehnung stärker abnimmt als der $E$-Modul und die Zugfestigkeit zunehmen. Erklären ließe sich dieser Sachverhalt eventuell damit, dass weniger Energie für das Fließen der Ketten aufgenommen werden kann. Die hier beschriebenen Ergebnisse Veränderungen der mechanischen Eigenschaften während des Vernetzens stehen im Einklang mit literaturbekannten Systemen. .91010227]

Abbildung 5.15 B zeigt die Zugkurven der unterschiedlich lang bestrahlten Proben des statistischen LMA-ANMA-Copolymers mit dem niedrigsten Anteil von $5 \%$ an ANMA. Diese Probe ist aufgrund des niedrigeren $T_{\mathrm{g}}$ so weich, dass sie erst nach 2 Stunden Bestrahlung eine ausreichende Festig- und Steifigkeit besitzt, um mittels Zugversuch untersucht werden zu können. Es treten mit zunehmender Bestrahlungsdauer die selben Trends in Bezug auf $E, \epsilon_{\mathrm{b}}, R_{\mathrm{m}}$ und $Z$ auf wie bei der vorherigen Probe. Bei gleicher Bestrahlungsdauer ist der $E$-Modul der Probe mit $5 \%$ ANMA niedriger als der der Probe mit $15 \%$, was auf den niedrigeren Gehalt an ANMA sowie den niedrigeren $T_{\mathrm{g}}$ zurückzuführen ist (Tabelle 5.9). Jedoch können auch bei einem Gehalt von nur $5 \%$ ANMA die mechanischen Eigenschaften in Form von Zugfestigkeit und $E$-Modul durch Bestrahlung immens verbessert werden.

Tab. 5.9. Übersicht der mittels Zugversuch ermittelten mechanischen Eigenschaften der Polymere $\mathrm{P}\left(\mathrm{LMA}_{0,85}-\mathrm{Co}-\mathrm{ANMA}_{0,15}\right.$ und $\mathrm{P}\left(\mathrm{LMA}_{0,95}-\mathrm{Co}-\mathrm{ANMA}_{0,05}\right)$ in Abhängigkeit der Bestrahlungsdauer $t$. Die angegeben Fehler sind die Standardabweichungen aus zwei Messungen.

\begin{tabular}{cccccc}
\hline Probe & $t / \mathrm{h}$ & $E / \mathrm{Mpa}$ & $\epsilon_{\mathrm{b}} / \%$ & $R_{\mathrm{m}} / \mathrm{MPa}$ & $Z / \mathrm{Mpa}$ \\
\hline $\mathrm{P}\left(\mathrm{LMA}_{0,85}\right.$-co-ANMA & \\
$0,15)$ & 4 & $63 \pm 6$ & $4,5 \pm 0,8$ & $0,88 \pm 0,16$ & $2,7 \pm 0,1$ \\
& 2 & $21,5 \pm 0,6$ & $26 \pm 4$ & $0,53 \pm 0,09$ & $8,4 \pm 0,6$ \\
& 1 & $10,9 \pm 0,3$ & $21,5 \pm 1,1$ & $0,55 \pm 0,05$ & $9,2 \pm 0,6$ \\
& 0,5 & $8,8 \pm 0,8$ & $54 \pm 8$ & $0,44 \pm 0,02$ & $15,2 \pm 0,8$ \\
& 0 & $\approx 0,5$ & - & $\approx 0,03$ & $\approx 17$ \\
\hline $\left.\mathrm{P}_{\left(\mathrm{LMA}_{0,95} \text {-co-ANMA }\right.}, 05\right)$ & 6 & $39 \pm 8$ & $4,6 \pm 0,2$ & $0,8 \pm 0,2$ & $1,4 \pm 0,4$ \\
& 4 & $7,7 \pm 0,8$ & $9,4 \pm 0,2$ & $0,24 \pm 0,04$ & $1,5 \pm 0,1$ \\
& 2 & $0,56 \pm 0,01$ & $32,9 \pm 0,1$ & $0,113 \pm 0,001$ & $2,31 \pm 0,08$ \\
\hline
\end{tabular}

Um zu bestimmen, wie hoch der Anteil der vernetzten Polymerketten ist, wurden die bestrahlten und vernetzten Probenkörper mit Toluol über mehrere Tage extrahiert, wobei das Lösungsmittel täglich gewechselt und aufgefangen wurde. Nach dem Entfernen des Lösungsmittel der Extraktionslösung und nach Trocknen des unlöslichen Polymers im Vakuumofen konnte so der Anteil an löslichem, unvernetztem Polymer bestimmt werden. 

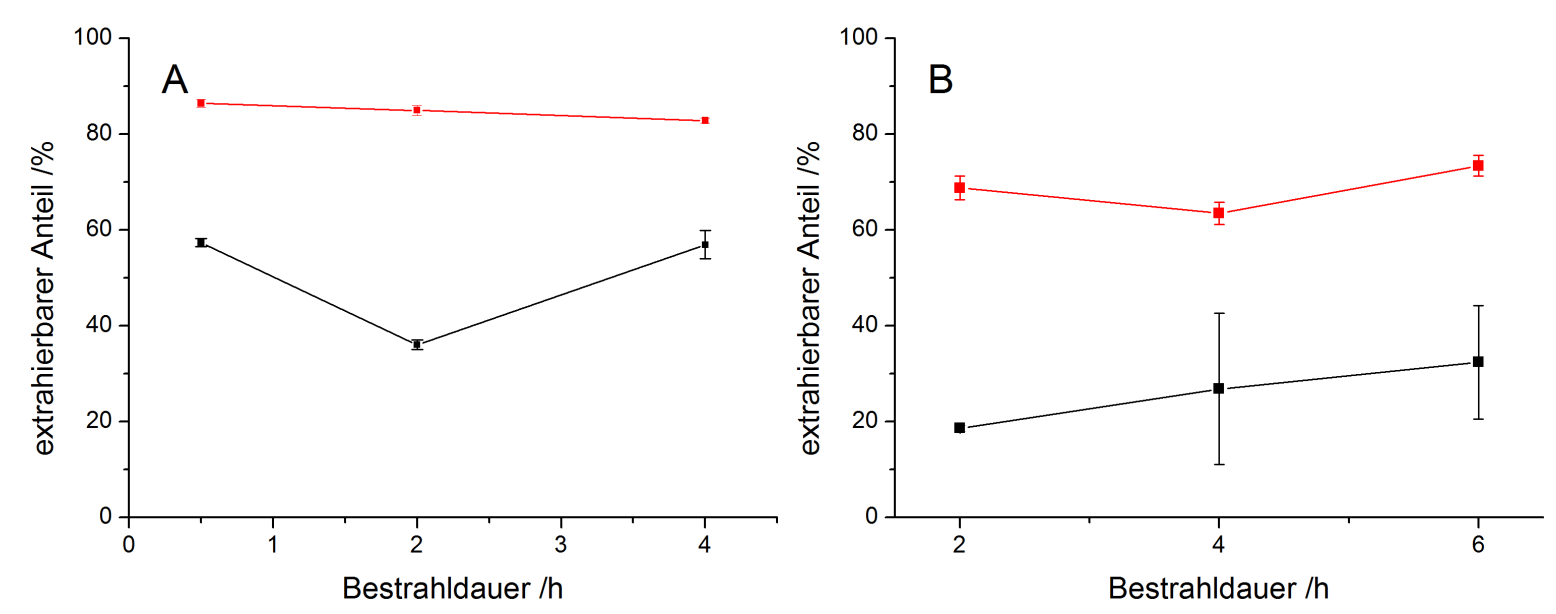

Abb. 5.16. Anteil des löslichen Polymers nach Vernetzung (schwarz) und nach Entnetzung (rot) für $\mathrm{P}\left(\mathrm{LMA}_{0,85}-\mathrm{CO}-\mathrm{ANMA}_{0,15}\right.$ (A) und $\mathrm{P}\left(\mathrm{LMA}_{0,95}-\mathrm{CO}-\mathrm{ANMA}_{0,05}\right)$ (B).

Abbildung 5.16 zeigt den Anteil an extrahierbaren Polymer für die statistischen Copolymere mit 5 und $15 \%$ ANMA direkt nach Bestrahlung der Probenkörper und nach der thermischen Entnetzung der bestrahlten Probenkörpern im Vakuumofen. Bei den vernetzten Polymeren beträgt der lösliche Anteil 20 bis $60 \%$. Die Vernetzung ist also nicht vollständig, was vermutlich darauf zurückzuführen ist, dass durch die hohe Absorption die Eindringtiefe des Lichtes begrenzt ist und somit das Material in der Mitte nicht vernetzt werden konnte. Der Anteil an vernetztem Polymer ist relativ unabhängig von den untersuchten Bestrahlungsdauern. Dies könnte ebenfalls auf die begrenzte Eindringtiefe des Lichtes zurückzuführen sein. Ein Teil der Anthracengruppen wird trotz Vernetzung nicht dimerisieren, da keine zweite Anthracengruppe in der Nähe ist. Zudem führt die Bestrahlung zur Verdunkelung des Probenkörpers (Abb. AA11, vermutlich aufgrund von Nebenreaktionen, wodurch mehr Strahlung absorbiert wird. Eine weitere Beobachtung ist, dass die Probe mit dem höheren Anteil ANMA einen geringeren Anteil an vernetztem Polymer besitzt. Dies ist zum einen auf die geringere Eindringtiefe der Strahlung durch vermehrte Absorption im Polymer mit $15 \%$ ANMA zurückzuführen. Zum anderen ist es durch den höheren Anteil an ANMA wahrscheinlicher, dass zwei Anthracengruppen einer Polymerkette in unmittelbarer Nähe zueinander liegen, wodurch die intramolekulare Dimerisierung bevorzugt wird. Bei beiden Polymeren steigt der Anteil an unvernetzten Polymer nach der thermischen Entnetzung signifikant an. Eine vollständige Entnetzung, wie es bei der Untersuchung dünner Filme im Abschnitt 5.3.4 möglich war, konnte für die dickeren Probenkörper aber nicht erreicht werden. Vermutlich führen die langen Bestrahldauern mit der intensiven Bestrahlung (1500 W-Lampe) und der fehlenden Inertgasatmosphäre zu Nebenreaktionen. Die Photooxidation von Anthracen zu Anthrachinon ist beispielsweise eine bekannte Nebenreaktion. 215-217

In Abbildung 5.17 sind in schwarz die Zugkurven des statistischen Copolymers mit $8 \%$ ANMA dargestellt. Die Veränderung der mechanischen Eigenschaften mit Bestrahlung folgen ebenfalls den Trends der Proben mit 5 und $15 \%$ ANMA. Nach dem Zugversuch wurden die bestrahlten und gerissenen Probenkörper im Vakuumofen thermisch entnetzt. 
Die entnetzten Probenkörper wurden dann mit Toluol extrahiert und nach dem Entfernen des Lösungsmittels wurden aus dem löslichen Anteil des recycelten Materials neue Probenkörper hergestellt. Die recycelten Probenkörper wurden erneut bestrahlt und mittels Zugversuch untersucht. Die Zugkurven des recycelten Materials wurden mit denen des ursprünglichen, nicht recycelten Materials bei gleicher Bestrahlungsdauer verglichen. Das recycelte Material reichte jedoch nur für einen Probenkörper je Bestrahlungsdauer, weshalb bei den Werten keine statistischen Fehler angegeben sind was entsprechend zu berücksichtigen ist. Wie in der Abbildung zu erkennen, ähneln die Zugkurven des recycelten Materials den ursprünglichen Zugkurven. Tabelle 5.10 zeigt auch, dass der E-Modul des recycelten Materials nahezu identisch mit dem ursprünglichen Material

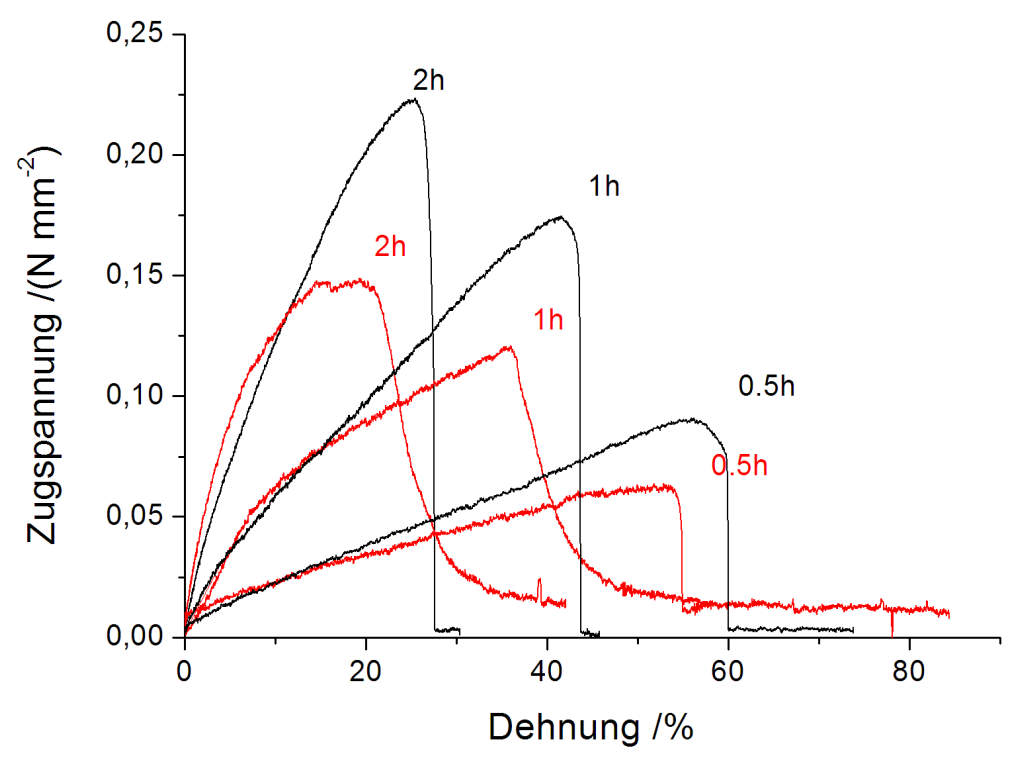

Abb. 5.17. Zugkurven des ursprünglichen (schwarz) und recycelten (rot) $P\left(L_{M A} A_{0,92}-c o-\right.$ $\mathrm{ANMA}_{0,08}$ ) nach unterschiedlicher Bestrahlungsdauer.

Tab. 5.10. Übersicht der anhand des Zugversuches ermittelten mechanischen Eigenschaften des ursprünglichen und recycelten Polymers $\mathrm{P}\left(\mathrm{LMA}_{0,92}-\mathrm{Co}-\mathrm{ANMA}_{0,08}\right)$ in Abhängigkeit der Bestrahlungsdauer $t$. Die angegeben Fehler sind die Standardabweichungen aus zwei Messungen. Vom recycelten Material konnte je Bestrahlungsdauer nur ein Probenkörper hergestellt werden.

\begin{tabular}{cccccc}
\hline Probe & $t / \mathrm{h}$ & $E / \mathrm{MPa}$ & $\epsilon_{\mathrm{b}} / \%$ & $R_{\mathrm{m}} / \mathrm{MPa}$ & $Z / \mathrm{MPa}$ \\
\hline ursprünglich & 2 & $1,75 \pm 0,07$ & $33 \pm 4$ & $0,22 \pm 0,01$ & $4,1 \pm 0,3$ \\
& 1 & $0,75 \pm 0,01$ & $47 \pm 2$ & $0,19 \pm 0,03$ & $4,9 \pm 0,6$ \\
& 0,5 & $0,18 \pm 0,04$ & $68 \pm 9$ & $0,11 \pm 0,02$ & $3,5 \pm 0,5$ \\
\hline recycelt & 2 & 2,2 & 42 & 0,15 & 3,9 \\
& 1 & 0,72 & 79 & 0,12 & 3,3 \\
& 0,5 & 0,16 & 58 & 0,06 & 2,3 \\
\hline
\end{tabular}


Tab. 5.11. Übersicht der anhand des Zugversuches ermittelten mechanischen Eigenschaften des Blockcopolymers $\mathrm{P}$ (BMA-co-MMA) ${ }_{10 \mathrm{k}} \mathrm{P}$ (LMA-co-ANMA) ${ }_{19 \mathrm{k}}$ in Abhängigkeit der Bestrahlungsdauer $t$. Die angegeben Fehler sind die Standardabweichungen aus zwei Messungen.

\begin{tabular}{ccccc}
\hline$t / \mathrm{h}$ & $E / \mathrm{MPa}$ & $\epsilon_{\mathrm{b}} / \%$ & $R_{\mathrm{m}} / \mathrm{MPa}$ & $Z / \mathrm{MPa}$ \\
\hline 4 & $32 \pm 6$ & $4,7 \pm 1,9$ & $0,77 \pm 0,05$ & $3,3 \pm 0,1$ \\
2 & $11,1 \pm 0,2$ & $76 \pm 2$ & $1,16 \pm 0,04$ & $64 \pm 3$ \\
0 & $1,3 \pm 0,3$ & $290 \pm 40$ & $0,27 \pm 0,02$ & $57 \pm 1$ \\
\hline
\end{tabular}

ist. Die ursprüngliche Zugfestigkeit und Zähigkeit wird aber nicht ganz erreicht, obwohl die Bruchdehnung leicht zunimmt. Nichtsdestotrotz konnte gezeigt werden, dass das vernetzte Material recycelt und durch erneute Bestrahlung wieder vernetzt werden kann.

\subsubsection{Untersuchung des Einflusses der Photovernetzung auf die mechanischen Eigenschaften der Blockcopolymere}

Der Einfluss der selektiven Photovernetzung innerhalb der weichen oder harten Phasen mikrophasenseparierter Blockcopolymere wurde ebenfalls untersucht. Die Blockcopolymere $\mathrm{P}$ (BMA-co-MMA) ${ }_{10 \mathrm{k}}-\mathrm{P}(\mathrm{LMA}-\mathrm{co}-\mathrm{ANMA})_{19 \mathrm{k}}$ und $\mathrm{PLMA}_{21 \mathrm{k}}-\mathrm{P}(\mathrm{BMA}-\mathrm{Co}-\mathrm{ANMA})_{21 \mathrm{k}}$ enthalten ANMA entweder im LMA- oder im BMA-Block. Probenkörper dieser BCP wurden anaolog zu den statistischen Polymeren mittels solvent casting hergestellt. Bei PLMA $21 \mathrm{k}-\mathrm{P}(\mathrm{BMA}-\mathrm{co}-$ ANMA) $)_{21 k}$ entstanden oftmals Blasen im Probenkörper, sodass zwei unbestrahlte Probenkörper aber jeweils nur ein mit zwei und vier Stunden bestrahlter Probenkörper hergestellt werden konnten. Dementsprechend können für die zwei und vier Stunden bestrahlen Probenkörper dieses Polymers keine Fehler der mechanischen Eigenschaften in Tabelle 5.12 angegeben werden.

Abbildung 5.18 zeigt die Zugkurven des BCP, das ANMA analog zu den zuvor untersuchten statistischen Copolymeren im LMA-Block enthält. Entsprechend sind die Trends der mechanischen Kennwerte mit zunehmender Bestrahlung ähnlich zu denen der statistischen Copolymer aus dem vorherigen Kapitel. Die Bruchdehnung nimmt mit zunehmender Bestrahlung ab und der E-Modul steigt (Tabelle 5.11). Der E-Modul und die Bruchdehnung des unbestrahlten Probenkörpers sind höher bzw. niedriger als bei dem statistischen Copolymer mit $15 \%$, was auf dem im BCP enthaltenden harten Block zurückzuführen ist. Die harte Phase des BMA-Blocks dient als physikalischer Vernetzungspunkt, sorgt damit für einen Erhöhung der Steifigkeit. Allerdings wird das aneinander vorbei Gleiten der Ketten eingeschränkt, wodurch die Bruchdehnung geringer ist, als bei dem erwähnten statistischen Copolymer. Diese Art von BCP mit einer weichen und harten Phase werden auch thermoplastischen Elastomeren genannt. [5+7]

Abbildung 5.19 zeigt die Zugkurven des Blockcopolymers, das ANMA im harten BMABlock enthält. Dieses BCP hat einen wesentlich längeren BMA-Block, als das BCP mit ANMA im LMA-Block. Der längere BMA-Block wird für die Ausbildung einer MPS benötigt und verändert die mechanischen Eigenschaften des BCP, sodass der E-Modul sowie die Zug- 


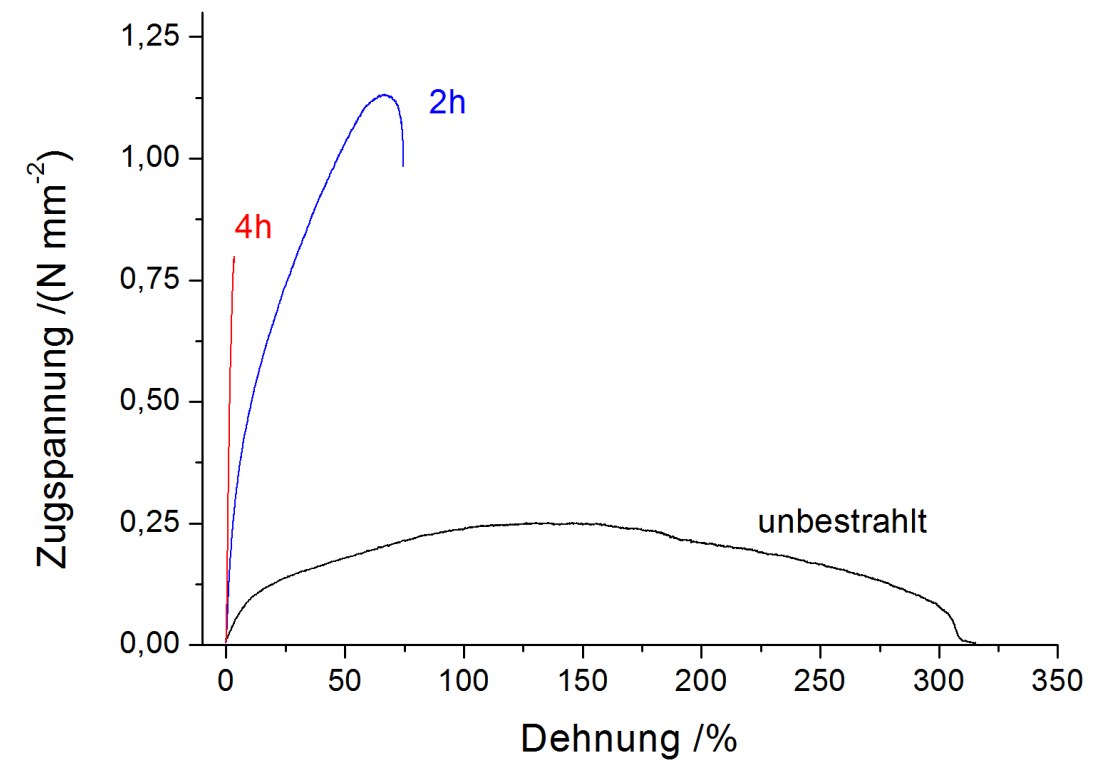

Abb. 5.18. Zugkurven des Blockcopolymers $P(B M A-c o-M M A){ }_{10 k}-P(\text { LMA-co-ANMA })_{19 k}$ nach unterschiedlicher Bestrahlungsdauer.

festigkeit schon wesentlich höher und die Bruchdehnung der unbestrahlten Probe niedriger sind als bei dem BCP in Abbildung 5.18. Ein starke Abhängigkeit der mechanischen Eigenschaften von der Zusammensetzung der Blockcopolymere wurde bereits in der Literatur beschrieben. 200[228] Während der Bestrahlung stieg die Temperatur der Bestrahlungskammer auf über den $T_{\mathrm{g}}$ auf größer $80^{\circ} \mathrm{C}$, sodass die Polymerketten beweglich genug für eine effiziente Vernetzung waren. Mit Bestrahlung des Probenkörpers steigen Zugfestigkeit und E-Modul im Gegensatz zum anderen BCP und den statistischen Copolymeren nicht oder nur minimal an. Die BMA-Phase des BCP ist bereits hart und eine Vernetzung erhöht die Steifigkeit der Probe daher kaum. Die Bruchdehnung und damit auch die Zähigkeit nehmen hingegen mit der Bestrahlung ab. Zu erwarten wäre eigentlich, dass die Bruchdehnung unabhängig von der Vernetzung innerhalb des harten Blockes ist, da während des Zuges hauptsächlich die weiche Phase gedehnt wird. Vermutlich wird jedoch durch die Vernetzungen in der harten Phase die Möglichkeit des aneinander Vorbeigleitens der Polymerketten beeinträchtigt, wodurch die Bruchdehnung reduziert wird.

Es konnte gezeigt werden, dass die Photovernetzung im LMA-Block, wie bereits bei den statistischen Copolymeren aus LMA und ANMA beobachtet, zu einer Erhöhung der Zugfestigkeit und des $E$-Moduls und zur Reduzierung der Bruchdehnung und der Zähigkeit führt. Die Photovernetzung im harten Bock reduziert ebenfalls die Bruchdehnung und die Zähigkeit, erhöht jedoch nicht oder nur kaum die Zugfestigkeit und den E-Modul. Daher ist die Photovernetzung im weichen Block für die Verbesserung der mechanischen Eigenschaften zu bevorzugen. 


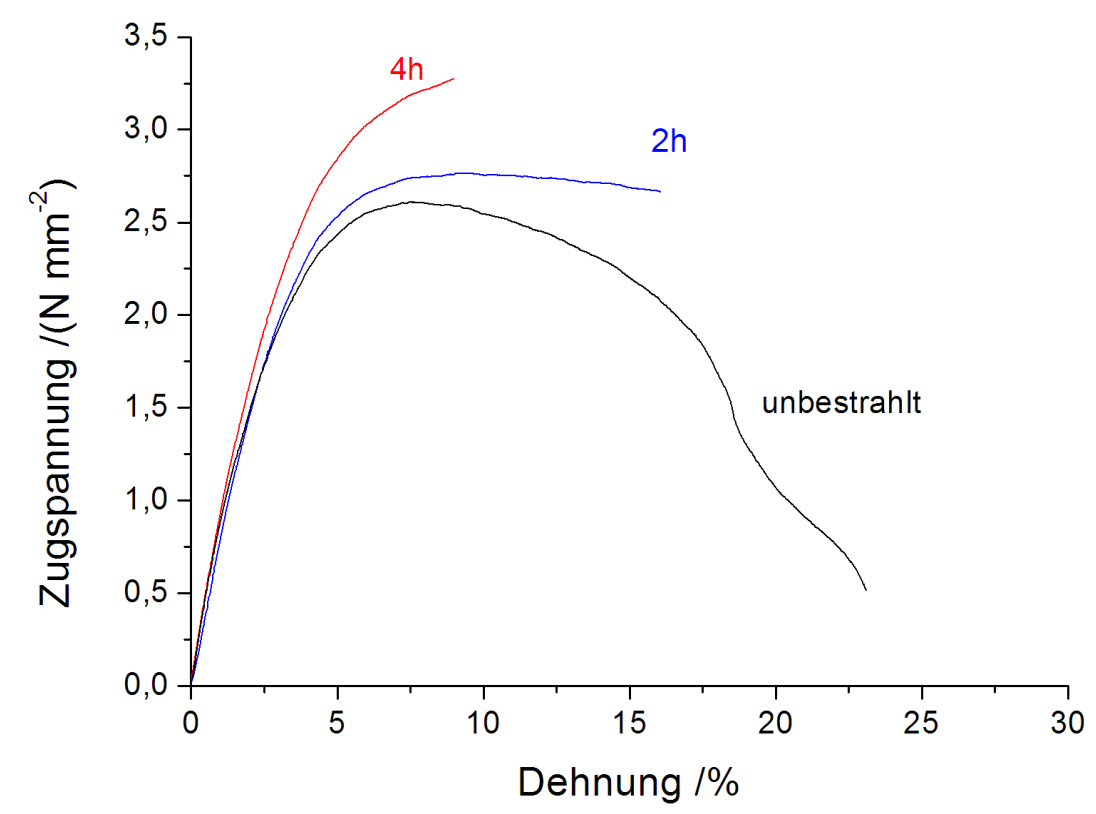

Abb. 5.19. Zugkurven des Blockcopolymers $P L M A_{21 k}-P(B M A-c o-A N M A)_{21 k}$ nach unterschiedlicher Bestrahlungsdauer.

Tab. 5.12. Übersicht der mittels Zugversuch ermittelten mechanischen Eigenschaften des Blockcopolymers $\mathrm{PLMA}_{21 \mathrm{k}}-\mathrm{P}(\mathrm{BMA}-\mathrm{co}-\mathrm{ANMA})_{21 \mathrm{k}}$ in Abhängigkeit der Bestrahlungsdauer $t$. Die angegeben Fehler sind die Standardabweichungen aus zwei Messungen.

\begin{tabular}{ccccc}
\hline$t / \mathrm{h}$ & $E / \mathrm{MPa}$ & $\epsilon_{\mathrm{b}} / \%$ & $R_{\mathrm{m}} / \mathrm{MPa}$ & $Z / \mathrm{MPa}$ \\
\hline 4 & 96 & 9,0 & $3,30,2$ & 21 \\
2 & 86 & 16 & 2,7 & 38 \\
0 & $89 \pm 6$ & $25 \pm 3$ & $2,6 \pm 0,3$ & $52 \pm 11$ \\
\hline
\end{tabular}

\subsection{Schlussfolgerung und Ausblick}

In diesem Projekt wurden photovernetzbare Blockcopolymere auf Basis von CMA und ANMA mittels RAFT-Polymerisation erfolgreich hergestellt. Ab einer mittleren Molmasse von 20 bis $24 \mathrm{~kg} \mathrm{~mol}^{-1}$ zeigen dünne Filme der CMA-enthaltenden BCP Mikrophasenseparation mit zylindrischer Morphologie. Während die Photovernetzung der Polymere mit CMA in dünnen Filmen bis zu hohen Umsätzen möglich war, erwies sich die photochemische Entnetzung als ineffektiv.

Die thermische Entnetzung der ANMA-Copolymere war hingegen in dünnen Filmen über mehrere Zyklen nahezu quantitativ. Mikrophasenseparation wurde auch in den ANMAenthaltenden LMA-BMA-Blockcopolymeren mittels AFM-Untersuchungen beobachtet. In diesem BCP-System hat eine geringe Veränderung der Zusammensetzung einen großen Einfluss auf den $\chi$-Parameter und die MPS. Die Anwesenheit einer weichen und harten 
Phase in den Blockcopolymeren bei Raumtemperatur konnte qualitativ mittels AFM und dem PFQNM-Modus sowie über die Ermittlung der Glasübergangstemperaturen bestätigt werden. Des Weiteren wurde anhand des Zugversuches an aus statistischen Copolymeren hergestellten Probenkörpern gezeigt, dass die Photovernetzung auch in bulk möglich ist und bereits ein kleiner Anteil an ANMA die mechanischen Eigenschaften in Form des $E$-Moduls um einen Faktor größer 100 verbessern kann. Dabei wurde bei kleineren Anteilen an ANMA mehr Polymer vernetzt, da das Licht für die photochemische Vernetzung aufgrund niedriger Absorption tiefer eindringen konnte. Zudem ist bei einem hohen Anteil an ANMA vermutlich die intramolekulare Dimerisierung der Anthracengruppen bevorzugt. Weiterhin konnte vernetztes Material erfolgreich durch thermische Entnetzung recycelt und erneut photovernetzt werden. Nach bestem Wissen des Autors wurden anhand der ANMA enthaltenden LMA-BMA-Blockcopolymer erstmals der Einfluss der selektiven Photovernetzung der weichen und harten Phase eines Blockcopolymers auf die mechanischen Eigenschaften untersucht. Es stellte sich heraus, dass die Photovernetzung der weichen Phase, analog zu den statistischen Copolymeren aus ANMA und LMA, zu einer Erhöhung des E-Moduls und einer Verkürzung der Bruchdehnung führt. Die Vernetzung der harten Phase bewirkte keine Veränderung des $E$-Moduls und eine Reduzierung der Bruchdehnung. Folglich ist die Vernetzung eines thermoplastischen Elastomers innerhalb der weichen Phase gegenüber der Vernetzung der harten Phase vorzuziehen, um die mechanischen Eigenschaften in Form des $E$-Moduls und der Zugfestigkeit zu erhöhen.

Es ist hier zu beachten, dass es sich bei der Untersuchung der mechanischen Eigenschaften um vorläufige Ergebnisse handelt, da aufgrund fehlender Zeit eine ausführliche Statistik durch Mittelung der Werte mehrerer Messung nicht immer möglich war. Der Herstellungsprozess für Probenkörper bei harten Polymeren wie z.B. dem Copolymer aus BMA und ANMA muss weiter optimiert werden, da weder mittels solvent casting noch mit dem Spritzgussverfahren blasenfrei bzw. unbeschädigte Probenkörper hergestellt werden konnten. Sowohl bei den Cumarin- als auch der Anthracen-enthaltenden Blockcopolymere wurde ein Ordnungs-Unordnungs-Übergang gefunden. Zudem konnte die MPS erfolgreich über die ODT-Temperatur hinaus durch Photovernetzung stabilisiert werden. Trotzdem bedarf die Untersuchung des ODT noch weiterer Untersuchungen, da nach dem ODT eine erneute Ausbildung der MPS nicht möglich war. Eventuell führen Nebenreaktionen bei den erhöhten Temperaturen zur Veränderungen des Polymers, möglicherweise auch nur an der Polymeroberfläche. Daher wäre es zukünftig ratsam, weitere Methoden, welche auch die tieferen Polymerschichten analysieren können, wie z.B. die Kleinwinkel-Röntgenstreuung (engl. smallangle $X$-ray scattering) zur Untersuchung des ODT zu verwenden. Die ANMA enthaltenden (Block)Copolymere könnten in Zukunft noch aufgrund inrer reversiblen Vernetzung auf ihre Selbstheilungseigenschaften untersucht werden. In der Literatur wurden bereits selbstheilende System basierend auf Cycloadditionsreaktionen beschrieben. ${ }^{[146 / 148 / 149]}$ Ebenso sollten diese BCP aufgrund ihrer Eigenschaft der Photovernetzung einen triple shape memory Effekt aufweisen, der zusätzlich noch analysiert werden könnte. 229-232] 


\section{Experimentalteil}

\subsection{Materialien}

Alle Chemikalien wurden - wenn nicht anders beschrieben - mit einer Reinheit von mindestens $95 \%$ von Sigma-Aldrich, ABCR, TCl Chemicals, fisher scientific / Acros, VWR, Roth oder Akzo Nobel bezogen. Trockene Lösungsmittel wurden durch Lagerung über aktiviertem Molsieb (über Nacht im Vakuumofen bei $180^{\circ} \mathrm{C}$ ) erhalten oder als trockenes Lösungsmittel kommerziell erworben. AIBN wurde aus Diethylether (ca. 3,0 g auf $85 \mathrm{~mL}$ ) umkristallisiert. Monomere wurden vor der Verwendung durch basisches Aluminiumoxid filtriert, um den Inhibitor zu entfernen.

\subsection{Verwendete Geräte und Probenherstellung}

\section{Rasterkraftmikroskopie und Probenherstellung}

AFM-Messungen wurden mit einem Multimode 8 AFM (Bruker) mit einem NanoScope V Regler und einem ScanAsyst-Air HR (Bruker, Federkonstante: $0,4 \mathrm{Nm}^{-1}$, Resonanzfrequenz: $70 \mathrm{kHz}$, Spitzenradius $2 \mathrm{~nm}$ ) oder FESPA (Bruker, Federkonstante $=2,8 \mathrm{Nm}^{-1}$, Resonanzfrequenz $\sim 75 \mathrm{kHz}$, Spitzenradius $=8 \mathrm{~nm}$ ) Cantilever bei Raumtemperatur gemessen. Die Messungen wurden entweder im PeakForce-Tapping ${ }^{\mathrm{TM}}$-Modus oder im PeakForce-Quantitative Nanomechanical Mapping ${ }^{\mathrm{TM}}$-Modus (PFQNM ${ }^{\mathrm{TM}}$ ) durchgeführt. Wenn nicht anders angegeben, wurden die Bilder mit einem Ausschnitt von 1,5 x 1,5 $\mu \mathrm{m}$, einer Rastergeschwindigkeit von $0,977 \mathrm{~Hz}$ (PFQNM ${ }^{\mathrm{TM}}$ ) oder 3,92 Hz (PeakForce-Tapping) und einer Auflösung von 512 x 512 Pixeln aufgenommen. Proben wurden durch spin coating nach dem Auftropfen von 5 bis $10 \mu \mathrm{L}$ einer 2,5 wt\%-igen Probenlösung auf ein Glasplättchen erhalten. Die Rotationsgeschwindigkeit betrug 1000 bis 2000 rpm. Nach dem spin coating wurden die Proben im Vakuumofen getrocknet und bei der angegebenen Temperatur über Nacht getempert. Die Schichtdicke betrug zwischen 90 und $200 \mathrm{~nm}$ und wurde freundlicherweise stichprobenartig mittels Ellipsometrie von Katharina Dabow bestimmt.

\section{Gelpermeationschromatographie}

(Meth-)Acrylate und Styrol

GPC-Analysen wurden mit Tetrahydrofuran als Eluent auf einem Agilent 1260 Infinity System bei $35{ }^{\circ} \mathrm{C}$ durchgeführt. Der Aufbau bestand aus einem Autosampler (Agilent 1260 Infinity Standard Autosampler), einer isokratischen Lösungsmittelpumpe (Agilent 1260 Infinity), einer PSS SDV-Vorsäule ( 8 × 50 mm, Partikelgröße $5 \mathrm{~mm}$ ), drei PSS SDV Hauptsäulen 
(8 x 300 mm, Partikelgröße: $5 \mu \mathrm{m}$, Porengröße: $10^{6}, 10^{5}$ und $10^{3} \AA$ A), einem Brechungsindexund einem UV/VIS-Detektor. Die Flussrate der mobilen Phase betrug $1 \mathrm{ml} \mathrm{min}^{-1}$. Das System wurde durch Polymethylmethacrylat- oder Polystyrol-Standards mit niedriger Dispersität und Toluol als internem Standard kalibriert. Die molaren Massen wurden entweder relativ zu PMMA oder nach dem Prinzip der universellen Kalibrierung (Mark-Houwink-Parameter: $a(\mathrm{BA})=0,700, K(\mathrm{BA})=0,0122 \mathrm{ml} \mathrm{g}^{-1} ; a(\mathrm{MA})=0,696, K(\mathrm{MA})=0,0168 \mathrm{ml} \mathrm{g}^{-1} ; a(\mathrm{DA})=$ $0,585, K(\mathrm{DA})=0,0292 \mathrm{ml} \mathrm{g}^{-1} ; a(\mathrm{DMA})=0,736, K(\mathrm{DMA})=0,0495 \mathrm{ml} \mathrm{g}^{-1} ; a(\mathrm{BMA})=0,664$, $\left.K(B M A)=0,0148 \mathrm{ml} \mathrm{g}^{-1}\right)$ bestimmt. 233/234 Alle Probenlösungen $\left(c=3 \mathrm{mg} \mathrm{ml}^{-1}\right)$ wurden vor der Injektion durch einen PTFE-Filter mit einer Porengröße von 0,45 $\mu \mathrm{m}$ filtriert.

\section{Hochtemperatur-Gelpermeationschromatographie (PE)}

HT-GPC-Analysen wurden mit 1,2,4-Trichlorobenzol als Eluenten bei $150{ }^{\circ} \mathrm{C}$ durchgeführt. Der Aufbau bestand aus einem Agilent G1888 Network Headspace Autosampler, einer isokratischen Lösungsmittelpumpe (Agilent 1260 Infinity), einem Agilent 1322A Entgaser und einer PSS 246 Schnittstelle, einer Polefin $10 \mu \mathrm{m}$ Vorsäule, drei Polefin Trennsäulen (Partikelgröße: $5 \mu \mathrm{m}$, Porengröße: $10^{6}, 10^{5}$ und $10^{3} \AA$ ) und einem Q 4 IR-Detektor. Das System wurde durch PE-Standards mit geringer und hoher Dispersität kalibriert.Alle Proben $\left(c=3 \mathrm{mg} \mathrm{ml}^{-1}\right)$ wurden in dem Eluenten mindestens eine Stunde vor der Messung gelöst.

\section{Kernspinresonanzspektroskopie}

NMR-Spektren wurden mit einem Varian Unity $300(300 \mathrm{MHz})$ von Varian bei Raumtemperatur oder im Falle von PE-Proben bei $90{ }^{\circ} \mathrm{C}$ gemessen. Als Lösungsmittel wurde deuteriertes Chloroform, Dichlormethan, Dimethylsulfoxid oder im Falle von PE-Proben Toluol verwendet. Der Peak des in Spuren vorhanden nicht deuterierten Lösungsmittels wurde zur Kalibrierung verwendet. Bei Polymeren wurde aufgrund der langsameren Bewegungen der großen Molekülen eine delay relaxation Zeit $\left(t_{2}\right)$ von $10 \mathrm{~s}$ gewählt, um das Einstellen der Boltzmann-Verteilung und korrekte Integrale zu gewährleisten. Die Auswertung erfolgte mit dem Programm SpinWorks 4 oder MestReNova. Die Multiplizitäten werden folgendermaßen abgekürzt: s (Singulett), d (Dublett), t (Triplett), q (Quartett), quin (Quintett), dd (Dublett von Dublett), m (Multiplett).

\section{UV-VIS-Absorptionsspektroskopie und Probenherstellung}

UV-VIS-Spektren von Lösungen wurden mit einem Cary 300 Scan Photospektrometer mit Quartz-Küvetten (Hellma Analytics QS $10 \mathrm{~mm}$ ) aufgenommen. Im Fall von auf Glasplättchen aufgetragenen Polymerfilmen konnten die Glasplättchen mit einem Durcmesser von $1 \mathrm{~cm}$ mittels eines extra angefertigten Halters direkt im Spektrometer untersucht werden. Für die Polymerfilme wurden mehrere Tropfen einer 2 bis 10\%-igen Polymerlösung auf die Glasplättchen aufgetropft und der Film im Vakuumofen getrocknet. Die Spektren der reinen Lösungsmittel oder des reinen Glasplättchens wurden jeweils als Hintergrund von den Spektren subtrahiert. 


\section{Thermogravimetrische Analyse}

Thermogravimetrische Analysen wurden mit einem Netzsch-TG- 209-F3-Tarsus-Gerät von Raumtemperatur bis $1000{ }^{\circ} \mathrm{C}$ und einer Heizrate von $10 \mathrm{~K} \mathrm{~min}^{-1}$ unter Luft durchgeführt.

\section{Dynamische Differenzkalorimetrie}

DSC-Messungen wurden mit der NETZSCH DSC 214 Polyma im Temperaturbereich von -100 bis $180^{\circ} \mathrm{C}$ mit einer Heiz- und Abkühlrate von $10 \mathrm{~K} \mathrm{~min}^{-1}$ unter konstantem Stickstoffstrom durchgeführt. Abkühlung erfolgte mittels flüssigem Stickstoff. Der Wärmefluss einer Polymerprobe wurde in einem Aluminiumtiegel gegen einen leeren Referenztiegel gemessen. Der $T_{\mathrm{g}}$ wurde über den Wendepunkte der Kurve bestimmt.

\section{Zugversuche}

Zugversuche wurde mit einer ZWICK \& ROELL Z2.5 Materialprüfmaschine bei $24 \pm 2{ }^{\circ} \mathrm{C}$ durchgeführt. Alle Messungen wurden mit einer Zuggeschwindigkeit von $25 \mathrm{~mm} \mathrm{~min}^{-1}$ durchgeführt. Je Probe wurden drei Messungen durchgeführt. In manchen Fällen war die Probenmenge nicht ausreichend, sodass nur zwei Messungen durchgeführt werden konnten. Zur Übersichtlichkeit wird nur eine repräsentative Kurve je Probe in den Auftragungen des Ergebnisteils dargestellt. Der E-Modul $(E)$, die Zugfestigkeit $\left(R_{\mathrm{m}}\right)$, die Bruchspannung $\left(\sigma_{\mathrm{B}}\right)$, die Zähigkeit $(Z)$, die Bruchdehnung $\left(\epsilon_{\mathrm{B}}\right)$ und die Streckgrenze $\left(R_{\mathrm{e}}\right)$ wurden mittels Origin 8.5G bestimmt.

\section{Dynamisch-mechanische Analyse}

Die in dieser Arbeit dargestellten DMA-Messungen wurden alle im Biegeversuch mit einer DMA 8000 von PerkinElmer durchgeführt. Zum Kühlen stand ein Autocryo System mit flüssigem Stickstoff und einem $50 \mathrm{~L}$ Dewar zur Verfügung. Alle Messungen wurden mit einer Frequenz von $1 \mathrm{~Hz}$, eine Heizrate von $2 \mathrm{~K} \mathrm{~min}^{-1}$ und einer dynamischen Auslenkung (strain) von $20 \mu \mathrm{m}$ durchgeführt. Proben wurden entweder in Form eines Probenkörpers oder als Pulver in einer Materialtasche gemessen. Wenn nicht anders angegeben, wurden die Proben im Temperaturintervall von -110 bis $100{ }^{\circ} \mathrm{C}$ analysiert.

\section{UV-Lampen, Bestrahlungs- und Entnetzungsexperimente}

Für die Bestrahlung der anthracenbasierten Probenkörper wurde die Bestrahlungskammer UVACUBE inert von der Firma Hönle verwendet. Diese wurde mit einer Quecksilberlampe (1500 W) ausgestattet. Auf dem Quarzglas der Bestrahlungskammer, also zwischen Lampe und Probe, wurde ein Borosilikatglas gegeben, welches als Langpassfilter fungiert und Wellenlängen < $320 \mathrm{~nm}$ ) blockt. 30 Minuten vor Beginn und während der Messung wurde ein leichter Argonstrom durch die Kammer geleitet. Nach der gewünschten Bestrahlzeit wurde der Probenkörper umgedreht, um inn gleichmäßig von beiden Seiten zu bestrahlen. Eine 
angegebene Bestrahlungsdauer von vier Stunden bedeutet, dass die Probe von oben und unten jeweils vier Stunden bestrahlt wurde. Die thermische Entnetzung der Proben fand im Ofen unter Vakuum bei $150-160^{\circ} \mathrm{C}$ über Nacht statt.

Dünne Filme der Polymerproben auf einem Glasplättchen wurden mit einer UV-Lampe XH 100, ausgestattet mit einer Quecksilberlampe (100 W), der Firma Müller bestrahlt. Die Bestrahlung der Cumarinproben fand in freier Atmosphäre statt. Für die Vernetzung wurde der Langpassfilter N-WG305 der Firma SCHOTT eingesetzt, der Wellenlängen unterhalb von $300 \mathrm{~nm}$ blockt blockt. Für die Entnetzung wurde ein Bandpassfilter, der nur Licht mit der $\lambda=254 \mathrm{~nm}$ durchlässt, in den Strahlengang der Lampe eingesetzt. Die Anthracenproben wurde photochemisch ebenfalls vernetzt. Dazu wurde ebenfalls der Langpassfilter N-WG305 verwendet. Die Proben wurden unter Argon in einem Glaskolben bestrahlt, wobei der Glaskolben selbst als zusätzlicher Langpassfilter fungiert und Wellenlängen unterhalb von 320 nm blockt. Die Entnetzung wurde nur thermisch in einem Kolben, gefüllt mir 0,5 bar Argon, durchgeführt. Dazu wurde der Kolben samt Probe im Ofen auf $150-160{ }^{\circ} \mathrm{C}$ über Nacht (12-15 h) getempert.

\section{MALDI-Massenspektrometrie}

Die MALDI-Messungen wurden mit einem Autoflex Speed Massenspektrometer von Bruker im Reflektronmodus durchgeführt, um eine möglichst hohe Massenauflösung zu erhalten. Das Spektrometer ist mit einem Nd:YAG Laser $(\lambda=355 \mathrm{~nm})$ ausgestattet. Trans-2-[3-(4-tertButylphenyl)-2-methyl-2-propenyliden]propandinitril (DCTB) diente als universelle Matrix für alle Polymere. Für PE wurde teils auch 2-(4-Hydroxyphenylazo)benzoesäure (HABA) als Matrix eingesetzt. Die Lösung des Polymers in Aceton $\left(c=10 \mathrm{mg} \mathrm{ml}^{-1}\right.$; bei PE: $5 \mathrm{mg} \mathrm{ml}^{-1}$ in Toluol), die Matrixlösung (40 mg ml-1 in THF) und bei Polymeren ohne ionische Endgruppe die Nal- oder AgTFA-Lösung (10 $\mathrm{mg} \mathrm{ml}^{-1}$ in THF) wurden im Verhältnis von 16:4 oder 20:4:4 gemischt. Im Falle von Endgruppen mit tertiären Aminen wurde statt des Salzes eine geringe Menge an Essigsäure zugegeben. Für die PE-Lösung wurde PE bei $90^{\circ} \mathrm{C}$ in Toluol gelöst (5 mg ml${ }^{-1}$ ). Die beim Abkühlen entstehende Suspension wurde dann mit der Marixlösung gemischt. Von der gemischten Lösung aus Matrix und Polymer (und gegebenenfalls Salz bzw. Essigsäure) wurde $1 \mu \mathrm{L}$ der Lösung auf die MALDI Probenplatte aus Edelstahl gegeben. Nach Verdampfen des Lösungsmittels bildete sich eine kristalline Schicht. Das MALDI-Spektrometer wurde extern mit einer PMMA Probe (als Natriumaddukt) bekannter Molmasse im Bereich von 1000 bis $2200 \mathrm{~m} / \mathrm{z}$ kalibriert. In der Gerätesoftware waren mehrere Einstellungsprofile für verschiedene $\mathrm{m} / \mathrm{z}$-Bereiche vorhanden. Bei diesen Profilen sind die Geräteparameter, wie die Detektorspannung sowie diverse Beschleunigungs- und Linsenspannungen, den entsprechenden Massenbereich angepasst. Das Einstellungsprofil wurde entsprechend der zu erwartenden Molmasse des jeweiligen Polymers gewählt. Jedes Spektrum ist die Summe aus 500 Laserschüssen. Die Spektren werden unbearbeitet dargestellt. Die Laserenergie wurde für jede Probe angepasst, um eine gute Massenauflösungen und intensive Signale zu erhalten. 


\section{ESI-MS}

ESI-MS von kleinen Molekülen wurden mit einem micrOTOF Massenspektrometer von Bruker mit einer Kapillarspannung von 4,5 kV von Mitarbeitern der zentralen Analytik der Universität Göttingen durchgeführt.

ESI-MS Messungen von Polymeren wurden mit einem Synapt G2 HDMS Massenspektrometer von Waters entweder im sensitivity oder im resolution Modus durchgeführt. Das Spektrometer wurde mit einer Lösung von $\mathrm{Nal}\left(0,5 \mathrm{mg} \mathrm{ml}^{-1}\right)$ in Aceton kalibriert.

Probenvorbereitungen und Geräteeinstellung für PE-Proben: PE wurde bei $90{ }^{\circ} \mathrm{C}$ in Dichlorethan $\left(5 \mathrm{mg} \mathrm{ml}^{-1}\right)$ gelöst. Nach dem Abkühlen wurde die Suspension durch einen Spritzenfilter mit einer Porengröße von 0,45 $\mu \mathrm{m}$ filtriert und die klare Lösung wurde 1:1 mit Acetonitril verdünnt. Im Falle des PE mit tertiärer Aminendgruppe wurde zusätzlich noch 0,1 wt\% Essigsäure hinzugegeben. Die so erhaltene Lösung wurde mit einer Flussrate von $300 \mu \mathrm{L} \mathrm{h}^{-1}$ und den folgenden Parametern in das Massenspektrometer eingespritzt: die ESI Source Temperatur betrug $120^{\circ} \mathrm{C}$, die Kapillarspannung 5,0 kV, die Desolvatisierungstemperatur $150{ }^{\circ} \mathrm{C}$, die Source Offset Spannung $80 \mathrm{~V}$ und die Cone Spannung $40 \mathrm{~V}$. Die Scanzeit betrug 0,5 s und jedes gezeigte Spektrum ist die Summe aus 120 Scans.

Acrylat- und Styrolproben wurden als 30 oder $50 \mu \mathrm{M}$ (bezogen auf den aus der GPC erhaltenen $\mathrm{M}_{n}$ ) Lösung in Aceton ins Massenspektrometer gesprüht. Im Falle der Polymere mit tertiärer Aminendgruppe wurde ebenfalls noch 0,1 wt\% Essigsäure hinzugegeben. Die Geräteparameter sind identisch mit denen der PE-Proben, außer dass die Kapillarspannung, wenn nicht anders angegeben, $3 \mathrm{kV}$ und die Desolvatisierungstemperatur $200^{\circ} \mathrm{C}$ betrug. Die Spektren wurden aus 120 oder 240 Scans aufsummiert. Das Signal-zu-Rausch-Verhältnis wurde am Peakmaximum bestimmt, indem die absolute Intensität des Signals durch das Rauschsignal geteilt wurde. Als Rauschsignal wurde das Maximum des Rauschens in unmittelbarer Nähe des Peakmaximums verwendet. Dies ist eine konservative Vorgehensweise. Die so erhaltenen Signal-zu-Rausch-Verhältnisse sind gegenüber der Verwendung der Standardabweichung des Rauschens als Rauschsignale um den Faktor 2 bis 6 niedriger.

\section{Probenkörperherstellung für Zugversuche und DMA}

Die Anthracencopolymere wurden unter Schütteln über mehrere Stunden in Dioxan ( 20 bis $30 \mathrm{wt} \%)$ gelöst. Die Lösungen wurden anschließend vorsichtig und blasenfrei in Knochenteflonnegativformen gegeben, die in der mechanischen Werkstatt der Universität Göttingen hergestellt wurden. Im Vakuumofen wurde dann bei etwa $85^{\circ} \mathrm{C}$ das Lösungsmittel entfernt. Dafür wurde der Druck am Ofen alle 0,5 bis 2 Stunden manuell in 100 mbar Schritten reduziert. Die Form wurde dann erneut mit der Polymerlösung aufgefüllt. Der Zyklus bestehend aus Auffüllen der Form und langsamen Entfernen des Lösungsmittels wurde so lange wiederholt, bis die Form nach Entfernen des Lösungsmittels vollständig gefüllt war. Die Teflonform samt Polymer wurden dann unter möglichst geringen Druck im Vakuumofen über Nacht auf $85^{\circ} \mathrm{C}$ temperiert, um auch die letzten Reste des Lösemittels zu entfernen. Die Teflonformen wurde dann für mehrere Stunden im Gefrierfach (Proben $P\left(L_{M A} A_{0,92}-C o-A N M A_{0,08}\right)$ und $\mathrm{P}\left(\mathrm{LMA}_{0,95}\right.$-co-ANMA $\left.\left.{ }_{0,05}\right)\right)$ oder im Kühlschrank $\left(\mathrm{P}\left(\mathrm{LMA}_{0,85}-\right.\right.$ co-ANMA $\left.\mathrm{A}_{0,15}\right)$ und $\mathrm{P}(\mathrm{BMA}-\mathrm{co}-$ 
MMA) ${ }_{10 k}-\mathrm{P}\left(\right.$ LMA-Co-ANMA) ${ }_{19 k}$ ) aufbewahrt. Nach der Herausnahme aus dem Kühlschrank und einer gegebenenfalls kurzen Wartezeit konnten die Probenkörper dann vorsichtig ohne Beschädigung aus der Form entnommen werden. Die probe PLMA ${ }_{21 k}-P(B M A-c o-A N M A)$ wurde bei Raumtemperatur der Teflonform entnommen. Die Dicke der Probenkörper betrug zwischen 1 und $1,5 \mathrm{~mm}$.

\subsection{Synthesen und Versuche: Projekt ionische Endgruppen}

\section{Synthese von PE mit lodendgruppe}

Die Synthese von PE und PE mit lodendgruppe (PE-I) wurde von Jannik Wagner mittels der CCG Polymerisation durchgeführt. Die Synthese ist in seiner Doktorarbeit oder in der gemeinsamen Publikation beschrieben. [150]

\section{Synthese von PE mit tertiärer Amin- oder Ammoniumendgruppe $\left(\mathrm{PE}-\mathrm{N} / \mathrm{N}^{+} / \mathrm{N}^{+}{ }_{6}\right)($ Schema 3.1)}

$\mathrm{Zu}$ einer $90{ }^{\circ} \mathrm{C}$ warmen Lösung von PE-I (1,0 äq, 10 wt\%) in Toluol wurde ein Überschuss (mindestens 3 äq) von 2-(Methylamino)ethanol, 3-Dimethylamino-1-propanol, oder 6-Dimethylamino-1-hexanol zugegeben. Die Lösung wurde für $48 \mathrm{~h}$ bei $90{ }^{\circ} \mathrm{C}$ geschüttelt. Nach Abkühlen auf Raumtemperatur wurde die entstandene Suspension filtriert und mehrmals mit Aceton gewaschen. Der Feststoff wurde erneut bei $90{ }^{\circ} \mathrm{C}$ in Toluol gelöst, durch Abkühlen gefällt, filtriert, mit Aceton gewaschen und im Vakuumofen über Nacht getrocknet; Ausbeute: etwa 85 - 90 \%. Die Zuordnung der ${ }^{1} \mathrm{H}$-NMR-Signale der Endgruppen wird im Abschnitt 3.2 in Abb. 3.2 beschrieben.

\section{Synthese von geladenem PE mit ATRP- oder Makromonomerfunktionalität (PE-N+-ATRP/-MA; Schema 3.8)}

$\mathrm{Zu}$ einer mit Argon entgasten Lösung von PE-N $\mathrm{N}^{+}$(1,0 äq, 10 wt\%), trockenem Triethylamin oder Pyridin (1,5 äq) und Toluol wurde bei $90{ }^{\circ} \mathrm{C}$ langsam eine Lösung von 2Brompropionsäurebromid oder Methacrylsäurechlorid (5 eq) zugetropft. Die Mischung wurde für $2 \mathrm{~h}$ bei $90{ }^{\circ} \mathrm{C}$ gerührt, auf Raumtemperatur abgekühlt, mit Methanol gequencht, filtriert, mehrmals mit Methanol gewaschen und im Vakuumofen über Nacht getrocknet. Die Zuordnung der NMR-Signale ist im Abschnitt 3.5 in Abb. 3.11 beschrieben.

\section{Synthese von PE-b-PBA mittels ATRP (Schema 3.9)}

Geladenes PE mit ATRP-Funktionalität (PE-N+-ATRP; 1 äq, 10 wt\% bezogen auf Toluol), trockenes Toluol, trockenes Anisol (10 wt\% bezogen auf Toluol), PMDETA (1,0 äq.) und CuBr ( 1,0 äq.) wurden in einem Schraubdeckelglas mit Septum mit Argon bei Raumtemperatur entgast. Entgastes Butylacrylate (100 äq) wurde hinzugegeben und die Mischung wurde 
auf $100{ }^{\circ} \mathrm{C}$ für $2 \mathrm{~h}$ im Heizblock geschüttelt. Die abgekühlte Lösung wurde in Methanol ausgefällt, filtriert, mehrmals mit Methanol und verdünnter Salzsäure gewaschen und im Vakuumofen über Nacht getrocknet.

\section{Synthese von PE-N+-RAFT durch nucleophile Substitution ausgehend von PE-I (Schema 3.6)}

Zu einer $90^{\circ} \mathrm{C}$ warmen Lösung von PE-I (1,0 äq; 10 wt\%) in Toluol:DMF (9:1) wurde ein Überschuss (8 äq) von RAFT-Amin zugegeben. Die Lösung wurde für $48 \mathrm{~h}$ bei $90{ }^{\circ} \mathrm{C}$ geschüttelt. Nach Abkühlen auf Raumtemperatur wurde die entstandene Suspension filtriert und mehrmals mit Aceton gewaschen. Der Feststoff wurde erneut bei $90{ }^{\circ} \mathrm{C}$ in Toluol gelöst, durch Abkühlen gefällt, filtriert, mit Aceton gewaschen und im Vakuumofen über Nacht getrocknet.

\section{Synthese von PE-N+'-RAFT oder PE-N-RAFT durch Veresterung von PE-N ${ }^{+}$ oder PE-N mit RAFT-COOH (Schema 3.7)}

Hier wird beispielhaft eine Steglichveresterung von PE-N+ beschrieben.

Dicyclohexylcarbodiimid (5 äq) und DMAP $(0,05$ äq) werden zu einer Suspension von PE-N ${ }^{+}$(1 äq, 5 wt\%) in Toluol gegeben. Nach Erwärmen auf $85^{\circ} \mathrm{C}$ wurde der Lösung RAFT$\mathrm{COOH}$ ( 5 äq) zugegeben und die Lösung wurde für $5 \mathrm{~h}$ bei $85^{\circ} \mathrm{C}$ geschüttelt. Nach Abkühlen auf Raumtemperatur wird die Suspension filtriert, mit Methanol gewaschen, erneut in Toluol bei $85{ }^{\circ} \mathrm{C}$ gelöst und durch Abkühlen gefällt. Nach dem Abfiltrieren wird der Feststoff mit Methanol gewaschen und im Vakuumofen getrocknet.

\section{Synthese von Cyanodekan mittels Phasentransferkatalyse}

Ein Phasentransferkatalysator (0.02 äq.; PE-N+ ${ }^{+}$, TPPB, TBAB, TBAl oder TOAB) wurde zu dem Zwei-Phasensystem bestehend aus Bromodekan (1,0 äq) in Oktan $(\sim 0,7 \mathrm{M})$ und $\mathrm{NaN}_{3}$ $(4,0$ äq) in Wasser $(\sim 2,7 \mathrm{M})$ gegeben und die Mischung wurde für 4 oder $18 \mathrm{~h}$ bei etwa $100^{\circ} \mathrm{C}\left(120-125^{\circ} \mathrm{C}\right.$ Ölbadtemperatur) gerührt. Nach Abkühlen auf Raumtemperatur wurde die organische Phase abgetrennt und das Lösungsmittel im Vakuum entfernt. Der Umsatz wurde mittels ${ }^{1} \mathrm{H}$-NMR-Spektroskopie anhand der Tripletts von Bromodekan ( $\delta=3,38 \mathrm{ppm}$ ) und Cyanodekan ( $\delta=2,28 \mathrm{ppm}$ ) bestimmt.

\section{Beladung von PE mit anionischem Methylorange}

PE-N $\mathrm{N}^{+}(\sim 50 \mathrm{mg})$ wurde in Toluol $(3 \mathrm{ml})$ bei $95^{\circ} \mathrm{C}$ gelöst. Die heiße Lösung wurde zu einer ebenfalls auf $95{ }^{\circ} \mathrm{C}$ erhitzten Lösung von Methylorange in Wasser (1,5 ml) gegeben. Das Zweiphasensystem wurde geschüttelt und die anschließend gefärbte organische Phase wurde abpipettiert und im Vakuum getrocknet, um das eingefärbte PE als orangen Feststoff zu erhalten. 
Zum Vergleich wurde analog mit kommerziellem PE verfahren, nur das TBAB (5-10 mg) hinzugefügt wurde, um so den Transfer des Methyloranges in die organische Phase zu gewährleisten.

\section{(RAFT-COOH)}

Die Synthese der Verbindung wurde in einer abgewandelter Form der Literaturvorschrift durchgeführt. 167]

$\mathrm{Zu}$ einer Suspension von $\mathrm{K}_{3} \mathrm{PO}_{4}(2,04 \mathrm{~g}, 13,2 \mathrm{mmol})$ in Aceton $(50 \mathrm{ml})$ wurde 1-Dodecanthiol (2,70 g, 13,2 mmol, 3,2 ml) zugegeben. Die Mischung wurde $10 \mathrm{~min}$ gerührt, mit Schwefelkohlenstoff $(2,74 \mathrm{~g}, 2,2 \mathrm{ml}, 36,0 \mathrm{mmol})$ versetzt und erneut für $60 \mathrm{~min}$ gerührt. Es wurde 2-Brompropionsäure (2,21 g, 14,4 mmol) zugegeben und über Nacht bei Raumtemperatur gerührt. Die Suspension wurde filtriert, der Filter mit Aceton gewaschen und das Filtrat wurde mit Salzsäure $(100 \mathrm{ml}, 1 \mathrm{M})$ versetzt und mit Diethylether $(2 \times 100 \mathrm{ml})$ extrahiert. Die organische Phase wurde mit Wasser $(75 \mathrm{ml})$ und gesättigter NaCl-Lösung $(75 \mathrm{ml})$ gewaschen. Der Diethylether wurde im Vakuum entfernt und der gelbe Feststoff aus Pentan oder Methanol umkristallisiert; Ausbeute: 3,10 g, 8,85 mmol (67\%).

${ }^{1} \mathrm{H}-\mathrm{NMR}\left(300 \mathrm{MHz}, \mathrm{CDCl}_{3}\right): \delta$ [ppm] = 0,88 (t, 3H, $\left.\mathrm{CH}_{3}-\mathrm{CH}_{3}\right), 1,15-1,46(\mathrm{~m}, 18 \mathrm{H}$, Alkylkette), $1.63\left(\mathrm{~d}, 3 \mathrm{H},-\mathrm{CH}-\mathrm{CH}_{3}\right), 1,71\left(\mathrm{~m}, 2 \mathrm{H} \mathrm{S}-\mathrm{CH}_{2}-\mathrm{CH}_{2}\right), 3.37\left(\mathrm{t}, 2 \mathrm{H}, \mathrm{S}-\mathrm{CH}_{2}\right), 4,87(\mathrm{q}, 1 \mathrm{H}$, $\mathrm{CH})$.

${ }^{13}$ C-NMR-Daten: siehe Literatur. ${ }^{167}$

\section{3-(Dimethylamino)propyl-2-(Dodecylthiocarbonothioylthio)propansäureester (RAFT-Amin)}

Eine Lösung von RAFT-COOH (500 mg, 1,43 mmol), 3-Dimethylamino-1-propanol (310 mg, $355 \mu \mathrm{L}, 3.00 \mathrm{mmol}$ ), 4-(Dimethylamino)-pyridin (4 mg, $33 \mu \mathrm{mol}$; DMAP) und Dicyclohexylcarbodiimid $(619 \mathrm{mg}, 3,00 \mathrm{mmol})$ in trockenem Dichlormethan $(10 \mathrm{ml}$; DCM) wurde bei Raumtemperatur über Nacht gerührt. Die entstandene Suspension wurde filtriert, der Filter mit DCM gewaschen und das Filtrat mit Wasser, 0,2 M Salzsäure, verdünnte Natronlauge $(0,1 \mathrm{M})$ und gesättigter $\mathrm{NaCl}$-Lösung (je $30 \mathrm{ml}$ ) gewaschen. Die organische Phase wurde abgetrennt und das DCM im Vakuum entfernt. Das erhaltene Öl wurde mittels Säulenchromatographie $\left(\mathrm{SiO}_{2}\right)$ aufgereinigt. Dafür wurden zuerst Nebenprodukte mit Ethylacetat:Pentan = 2:1 eluiert und verworfen und dann die gelbe Fraktion des Produktes mit Ethylacetat:Pentan:Triethylamin = 1:2:0,015 eluiert und aufgefangen. Das Lösungsmittel wurde im Vakuum entfernt und das gelb-orangene Produkt als Öl erhalten und im Feinvakuum getrocknet; Ausbeute: $350 \mathrm{mg}, 803 \mu \mathrm{mol}$ (56 \%).

${ }^{1} \mathrm{H}-\mathrm{NMR}\left(300 \mathrm{MHz}, \mathrm{CDCl}_{3}\right): \delta[\mathrm{ppm}]=4,82(\mathrm{q}, 1 \mathrm{H}, \mathrm{CH}), 4,19\left(\mathrm{t}, 2 \mathrm{H}, \mathrm{OCH}_{2}\right), 3,34(\mathrm{t}, 2 \mathrm{H}$, $\left.\mathrm{SCH}_{2}\right), 2,32\left(\mathrm{t}, 2 \mathrm{H}, \mathrm{CH}_{2} \mathrm{~N}\right), 2,22\left(\mathrm{~s}, 6 \mathrm{H},\left(\mathrm{CH}_{3}\right)_{2} \mathrm{~N}\right), 1,80\left(\mathrm{~m}, 2 \mathrm{H}, \mathrm{CH}_{2} \mathrm{CH}_{2} \mathrm{O}\right), 1,69(\mathrm{~m}, 2 \mathrm{H}$, $\left.\mathrm{CH}_{2} \mathrm{CH}_{2} \mathrm{~S}\right), 1,60$ (d, 3H, $\left.\mathrm{CH}_{3} \mathrm{CH}\right), 1,16-1,45$ (m, 18H, Alkylkette), 0,88 (t, 3H, $\left.\mathrm{CH}_{3}\left(\mathrm{CH}_{2}\right)_{11}\right)$.

${ }^{13} \mathrm{C}-\mathrm{NMR}\left(75 \mathrm{MHz}, \mathrm{CDCl}_{3}\right): \delta[\mathrm{ppm}]=222,1(\mathrm{C}=\mathrm{S}), 171,1(\mathrm{C}=\mathrm{O}), 64,1\left(\mathrm{CH}_{2}-\mathrm{O}\right), 56,0$ $\left(\mathrm{CH}_{2}-\mathrm{N}\right), 47,9(\mathrm{SCH}), 45,4\left(2 \times \mathrm{CH}_{3}-\mathrm{N}\right), 37,2\left(\mathrm{CH}_{2}-\mathrm{S}\right)$, restliche $\mathrm{CH}_{2}: 31,9,29,6$, 
29,5, 29,4, 29,3, 29,1, 28,9, 27,8; 26,7 $\left(\mathrm{CH}_{2} \mathrm{CH}_{2}-\mathrm{O}\right), 22.7\left(\mathrm{CH}_{3} \mathrm{CH}_{2}\right), 16.8\left(\mathrm{CH}_{3} \mathrm{CH}\right), 14.1$ $\left(\mathrm{CH}_{3} \mathrm{CH}_{2}\right)$.

ESI-MS (positiver Modus, $\mathrm{m} / \mathrm{z}$ ): gemessen: 436,2375 $\left([\mathrm{M}+\mathrm{H}]^{+}\right)$, berechnet: 436,2372 $\left([\mathrm{M}+\mathrm{H}]^{+}\right)$.

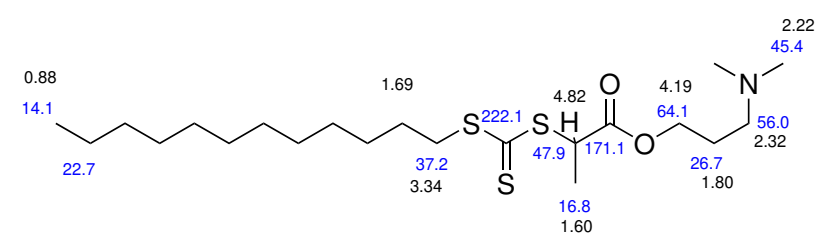

Abb. 6.1. Struktur und Zuordnung der NMR-Signale (in ppm) von RAFT-Amin (blau: ${ }^{13} \mathrm{C}$, schwarz: $\left.{ }^{1} \mathrm{H}\right)$.

\section{3-((2-(dodecylthiocarbonothioylthio)propanoyl)oxy)-N,N,N-trimethylpropan-1- ammoniumiodid (RAFT-N ${ }^{+}$)}

Eine Lösung von RAFT-Amin (2,17 g, 4,98 mmol) und Methyliodid $(2,13 \mathrm{~g}, 15 \mathrm{mmol})$ in Diethylether $(50 \mathrm{ml})$ wurde für $24 \mathrm{~h}$ bei Raumtemperatur gerührt. Der ausgefallene Feststoff wurde abfiltriert, mit Diethylether gewaschen und im Feinvakuum getrocknet. Das so erhaltene Produkt $(2,84 \mathrm{~g}, 4,91 \mathrm{mmol}, 98 \%)$ ist bereits sauber, kann aber bei Bedarf noch aus Aceton umkristallisiert werden (ca. $105 \mathrm{ml}$ Aceton auf 2,7 g; Ausbeute: 2,02 g).

${ }^{1} \mathrm{H}-\mathrm{NMR}\left(300 \mathrm{MHz}, \mathrm{CDCl}_{3}\right): \delta[\mathrm{ppm}]=4,78(\mathrm{q}, 1 \mathrm{H}, \mathrm{CH}), 4,17\left(\mathrm{t}, 2 \mathrm{H}, \mathrm{OCH}_{2}\right), 3,39(\mathrm{t}, 2 \mathrm{H}$, $\left.\mathrm{SCH}_{2}\right), 3,38\left(\mathrm{t}, 2 \mathrm{H}, \mathrm{CH}_{2} \mathrm{~N}\right), 3,09\left(\mathrm{~s}, 9 \mathrm{H}, \mathrm{N}-\left(\mathrm{CH}_{3}\right)_{3} 2,08\left(\mathrm{~m}, 2 \mathrm{H}, \mathrm{CH}_{2} \mathrm{CH}_{2} \mathrm{O}\right), 1,64(\mathrm{~m}, 2 \mathrm{H}\right.$, $\mathrm{CH}_{2} \mathrm{CH}_{2} \mathrm{~S}$ ), 1,56 (d, 3H, $\mathrm{CH}_{3} \mathrm{CH}$ ), 1,13-1,42 (m, 18H, Alkylkette), 0,88 (t, 3H, $\left.\mathrm{CH}_{3}\left(\mathrm{CH}_{2}\right)_{11}\right)$.

${ }^{13} \mathrm{C}-\mathrm{NMR}\left(75 \mathrm{MHz}, \mathrm{CDCl}_{3}\right): \delta[\mathrm{ppm}]=222,1(\mathrm{C}=\mathrm{S}), 171,0(\mathrm{C}=\mathrm{O}), 62,51\left(\mathrm{CH}_{2}-\mathrm{N}\right)$, 62,46 $\left(\mathrm{CH}_{2}-\mathrm{O}\right), 52,3\left(\mathrm{CH}_{3}-\mathrm{N}\right), 47,6(\mathrm{~S}-\mathrm{CH}), 36,6\left(\mathrm{CH}_{2}-\mathrm{S}\right), 27,4 \mathrm{CH}_{2} \mathrm{CH}_{2} \mathrm{~S}, 22,04$ $\left(\mathrm{CH}_{3}-\mathrm{CH}_{2}-\mathrm{Alkyl}\right), 21,97\left(\mathrm{O}-\mathrm{CH}_{2}-\mathrm{CH}_{2}\right), 13,9\left(\mathrm{CH}_{3}-\right.$ Alkyl); restliche $\mathrm{CH}_{2}: 31,2,28,94$, 28,86, 28,76, 28,64, 28,37, 28,10.

ESI-MS (positiver Modus, m/z): gemessen: 450,2531 ([M-Br $]^{+}$), berechnet: 450,2529 ([M$\left.\mathrm{Br}]^{+}\right)$.

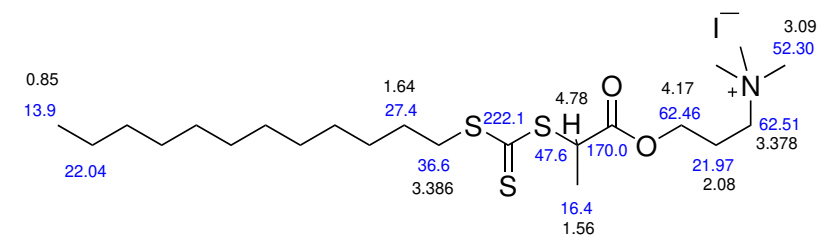

Abb. 6.2. Struktur und Zuordnung der NMR-Signale (in ppm) von RAFT-N ${ }^{+}$(blau: ${ }^{13} \mathrm{C}$, schwarz: $\left.{ }^{1} \mathrm{H}\right)$.

\section{Synthese von 4-(Bromomethyl)benzyltriethylammoniumbromid}

Die Synthese wurde entsprechend der Literatur durchgeführt. 153 
Triethylamin (1,43 g, 14,2 mmol) und $\alpha$, $\alpha^{\prime}$-Dibrom- $p$-xylol (3,30 g, 12,5 mmol) wurden in Ethylacetat $(45 \mathrm{ml})$ suspendiert und bei $50{ }^{\circ} \mathrm{C}$ für $48 \mathrm{~h}$ gerührt. Das Produkt wurde abfiltriert, mit Ethylacetat $(15 \mathrm{ml})$ gewaschen und im Feinvakuum getrocknet; Ausbeute: $4,54 \mathrm{~g}$, $12,4 \mathrm{mmol}(99 \%)$.

${ }^{1} \mathrm{H}-\mathrm{NMR}\left(300 \mathrm{MHz}, \mathrm{CDCl}_{3}\right): \delta[\mathrm{ppm}]=7,61\left(\mathrm{~d}, 2 \mathrm{H}, \mathrm{BR}-\mathrm{CH}_{2}-\mathrm{C}-\left(\mathrm{CH}_{\text {arom }}\right)\right), 7,45(\mathrm{~d}, 2 \mathrm{H}$, $\left.\mathrm{N}-\mathrm{CH}_{2}-\mathrm{C}-\left(\mathrm{CH}_{\text {arom }}\right)\right), 4,91$ (s, 2H, N- $\mathrm{CH}_{2}-$ Phenyl)), 4,47 (s, 2H, Br- $\mathrm{CH}_{2}-$ Phenyl)), 3,44 (q, 6H, N-( $\left.\left.\mathrm{CH}_{2}\right)_{3}\right), 1,46\left(\mathrm{t}, 9 \mathrm{H}, \mathrm{N}-\left(\mathrm{CH}_{2}-\mathrm{CH}_{3}\right)_{3}\right)$.

${ }^{13} \mathrm{C}$-NMR-Daten: siehe Literatur. ${ }^{153}$

\section{3-((2-(dodecylthiocarbonothioylthio)propanoyl)oxy)-N,N-dimethyl-N-(4-((tri- ethylammoniumbromid)methyl)benzyl)propan-1-ammoniumiodid (RAFT- ${ }^{2+}$ )}

RAFT-Amin (500 mg, 1,15 mmol) und 4-(Bromomethyl)benzyltriethylammoniumbromid (420 mg, 1,15 mmol) wurden in DCM $(5 \mathrm{ml})$ gelöst und für $48 \mathrm{~h}$ bei Raumtemperatur gerührt. Die Lösung wurde mit Diethylether $(25 \mathrm{ml})$ versetzt und der ausgefallene, hygroskopische Feststoff wurde abfiltriert und im Feinvakuum getrocknet; Ausbeute: $890 \mathrm{mg}, 1,11 \mathrm{mmol}$ (96\%).

${ }^{1} \mathrm{H}-\mathrm{NMR}\left(300 \mathrm{MHz}, \mathrm{CD}_{2} \mathrm{Cl}_{2}\right): \delta[\mathrm{ppm}]=7,74(\mathrm{~s}, 4 \mathrm{H}$, aromatische $\mathrm{CH}), 5,25(\mathrm{~s}, 2 \mathrm{H}$, $\mathrm{N}-\mathrm{CH}-$ Phenyl) , 5,04 (s, 2H, N-CH-Phenyl), 4,79 (q, $1 \mathrm{H}, \mathrm{CH}), 4,27$ (t, 2H, O- $\mathrm{CH}_{2}$ ), $3,68\left(\mathrm{t}, 2 \mathrm{H}, \mathrm{N}-\mathrm{CH}_{2}-\mathrm{CH}_{2}\right), 3,46-3,30\left(\mathrm{~m}, 8 \mathrm{H}, \mathrm{S}-\mathrm{CH}_{2}, \mathrm{~N}-\left(\mathrm{CH}_{2}\right)_{3}\right), 3,27\left(\mathrm{~s}, 6 \mathrm{H}, \mathrm{N}-\left(\mathrm{CH}_{3}\right)_{2}\right)$, 2,30 (m, 2H, O- $\left.\mathrm{CH}_{2}-\mathrm{CH}_{2}\right), 1,68\left(\mathrm{~m}, 2 \mathrm{H}, \mathrm{S}-\mathrm{CH}_{2}-\mathrm{CH}_{2}\right), 1,61\left(\mathrm{~d}, 3 \mathrm{H}, \mathrm{CH}_{3}-\mathrm{CH}\right), 1,50(\mathrm{t}, 9 \mathrm{H}$, $\left.\mathrm{N}-\left(\mathrm{CH}_{2}-\mathrm{CH}_{3}\right)_{3}\right), 1,43-1,19\left(\mathrm{~m}, 18 \mathrm{H}\right.$, Rest Alkylkette), 0,88 (t, 3H, $\left.\mathrm{CH}_{3}\left(\mathrm{CH}_{2}\right)_{11}\right)$.

${ }^{13} \mathrm{C}-\mathrm{NMR}\left(75 \mathrm{MHz}, \mathrm{CDCl}_{3}\right): \delta[\mathrm{ppm}]=223,4(\mathrm{C}=\mathrm{S}), 170,8(\mathrm{C}=\mathrm{O}), 134,1\left(\mathrm{CH}_{\text {aromat }}\right), 133,6$ $\left(\mathrm{CH}_{\text {aromat }}\right), 129,8\left(\mathrm{C}_{\mathrm{q} \text {, aromat }}\right), 129,6\left(\mathrm{C}_{\mathrm{q} \text {, aromat }}\right) .66,2\left(\mathrm{~N}-\mathrm{CH}_{2}-\mathrm{C}_{\text {aromat }}\right), 62,4\left(\mathrm{O}-\mathrm{CH}_{2}\right), 61,3$ $\left.\left(\mathrm{N}-\mathrm{CH}_{2}-\mathrm{CH}_{2}\right), 60,3\left(\mathrm{~N}-\mathrm{CH}_{2}-\mathrm{C}_{\text {aromat }}\right), 52,2\left(\mathrm{~N}_{-} \mathrm{CH}_{2}-\mathrm{CH}_{3}\right), 49,9 \mathrm{~N}-\mathrm{CH}_{3}\right), 47,9(\mathrm{~S}-\mathrm{CH}), 37,4$ (S$\left.\mathrm{CH}_{2}\right), 27,8\left(\mathrm{~S}-\mathrm{CH}_{2}-\mathrm{CH}_{2}\right), 22,04\left(\mathrm{CH}_{2}-\mathrm{CH}_{3}\right)$ 16,7, $14,1\left(\mathrm{CH}_{2}-\mathrm{CH}_{3}\right), 8,6\left(\mathrm{~N}-\mathrm{CH}_{2}-\mathrm{CH}_{3}\right)$; restliche Cs der Alkylkette: 31,8, 29,56, 29,54, 29,49, 29,25, 29,06, 28,88, 22,6.

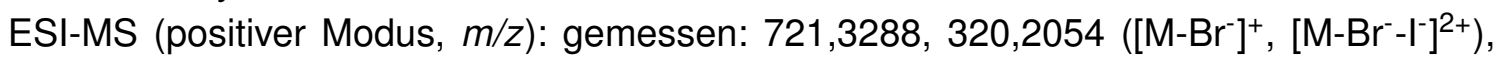
berechnet: $721,3289,320,2059\left(\left[\mathrm{M}-\mathrm{Br}^{-}\right]^{+},\left[\mathrm{M}-\mathrm{Br}^{-}-\mathrm{I}^{-}\right]^{2+}\right)$.

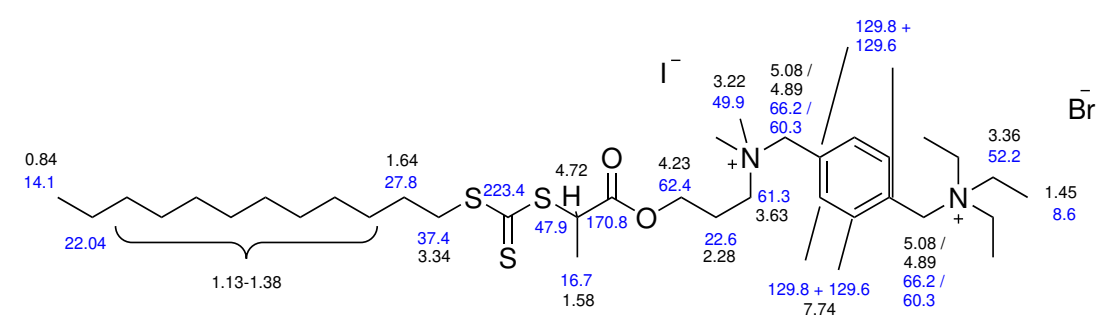

Abb. 6.3. Struktur und Zuordnung der NMR-Signale von RAFT-N ${ }^{2+}$ (blau: ${ }^{13} \mathrm{C}$, schwarz: ${ }^{1} \mathrm{H}$ ).

\section{Allgemeine Vorschrift für die RAFT-Polymersynthesen aus dem Kapitel 4}

AIBN wurde für MA und Styrol als Initiator verwendet und die Polymerisation wurde bei $60{ }^{\circ} \mathrm{C}$ (MA, Styrol) oder $70^{\circ} \mathrm{C}$ (Styrol) durchgeführt. Das Verhältnis von RAFT-Agens zu 
AIBN betrug in allen Fällen 10:1. Als Lösungsmittel wurde DMF (RAFT-N ${ }^{+}$) oder Butanon (RAFT-COOH/-Amin) im Verhältnis 1:1 zum Monomer verwendet. Im Falle der Polymerisation mit RAFT-N ${ }^{2+}$ wurde das Monomer mit dem 1,7-fachem Volumen von DMF und mit so wenig wie möglich Wasser verdünnt. Genauere Details der einzelnen Synthesen finden sich im Abschnitt 4.3 .

In einem Schraubdeckelglas wurden RAFT-Agens und AIBN im Lösungsmittel gelöst. Nach Zugabe des Monomers wurde die Lösung durch ein Septum für mindestens 10 Minuten mit Argon gespült. Das Schraubdeckelglas wurde im vortemperierten Heizblock bei 60 oder $70{ }^{\circ} \mathrm{C}$ geschüttelt. Nach der angegeben Zeit (Tabelle 4.1 oder 4.2) wurde das Gefäß entnommen und mit Eis oder mit kaltem Wasser von außen abgekühlt. Die Reaktionslösung wurde in eine Aluschale gegeben und das Lösungsmittel und Rest Monomer über Nacht abgedampft. Anschließend wurde das Polymer im Vakuum getrocknet.

\section{Methylierung der Dimethylamino-Gruppe in Polymeren}

Etwa 40 - 60 mg des mit dem RAFT-Agens RAFT-Amin hergestellten Polymers, Chloroform $(\sim 400 \mu \mathrm{L})$ und Mel (10 - 30 äq) wurden für $48 \mathrm{~h}$ in einem verschlossenen Gefäß gerührt oder geschüttelt. Das Lösemittel und der Überschuss an Mel wurde in einem gut belüfteten Abzug durch Abdampfen entfernt. Die Analyse der Reaktion ist in Abschnitt 4.3 beschrieben.

\section{Synthese von 9-Anthracenylmethylmethacrylat (ANMA)}

Die Synthese der Verbindung wurde in einer abgewandelter Form der Literaturvorschrift durchgeführt. 204]

Eine Lösung von Methacrylsäurechlorid $(5,01 \mathrm{~g}, 48.0 \mathrm{mmol})$ in trockenem DCM $(25 \mathrm{ml})$ wurde tropfenweise bei $0{ }^{\circ} \mathrm{C}$ zu einer Lösungen von 9 -Hydroxymethylanthracen $(5,00 \mathrm{~g}$, $24,0 \mathrm{mmol})$ und Triethylamin $(2,42 \mathrm{~g}, 24,0 \mathrm{mmol})$ in trockenem DCM $(50 \mathrm{ml})$ gegeben. Die Lösung wurde für $2 \mathrm{~h}$ bei $0^{\circ} \mathrm{C}$ und anschließend über Nacht bei Raumtemperatur gerührt. Die Lösung wurde mit wässriger $\mathrm{NaOH}$-Lösung (2 M, 3×100 ml), Wasser (100 ml) und gesättigter NaCl-Lösung (100 ml) gewaschen. Die organische Phase wurde abgetrennt, eingeengt und säulenchromatographisch $\left(\mathrm{SiO}_{2}\right.$, Gradient von Ethylacetat:Pentan 1:2 zu Ethylacetat:Pentan 1/:1) aufgereinigt. Die das Produkt enthaltenden, gelben Fraktionen wurden vereinigt und das Lösungsmittel wurde im Vakuum entfernt. Das Produkt kristallisiert entweder im Hochvakuum oder im Kühlschrank aus; Ausbeute: 6,05 g, 21,9 mmol (91\%).

${ }^{1} \mathrm{H}-\mathrm{NMR}\left(300 \mathrm{MHz}, \mathrm{CDCl}_{3}\right): \delta[\mathrm{ppm}]=8,50\left(\mathrm{~s}, 1 \mathrm{H}, \mathrm{CH}-\mathrm{Ar}_{10}\right), 8,36\left(\mathrm{~d}, 2 \mathrm{H}, \mathrm{CH}_{\text {aromat:1, }}\right)$,

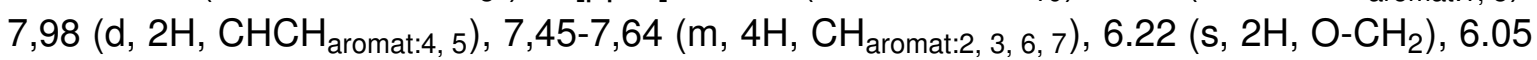
(s, $\left.1 \mathrm{H}, \mathrm{C}=\mathrm{CH}_{2}\right), 5.50\left(\mathrm{~s}, 1 \mathrm{H}, \mathrm{C}=\mathrm{CH}_{2}\right), 1.91\left(\mathrm{~s}, 3 \mathrm{H}, \mathrm{CH}_{3}\right)$.

${ }^{13} \mathrm{C}-N M R-D a t e n:$ siehe Literatur. 204] 


\section{Synthese von 7-(2-Hydroxyethoxy)-4-methylcumarin}

Die Verbindung ist bereits bekannt und die ${ }^{13} \mathrm{C}-\mathrm{NMR}$ Daten sind in der Literatur zu finden. 198

7-Hydroxy-4-methylcumarin (4,40 g, 25,0 mmol), Kaliumcarbonat (5,00 g, 37,5 mmol), Kaliumiodid (260 mg, 1,57 mmol) und Chlorethanol (4,03 g, 50,0 mmol) wurden über Nacht bei $110^{\circ} \mathrm{C}$ in DMF (50 ml) gerührt. Nach Abkühlen auf Raumtemperatur wurde der Mischung Wasser (50 ml) zugegeben, wodurch über Nacht ein weißer Feststoff auskristallisierte. Der Feststoff wurde abfiltriert, mit Wasser gewaschen und und aus Ethanol/Hexan (7/1) umkristallisiert; Ausbeute: 4,39 g, 20,0 mmol (80 \%).

${ }^{1} \mathrm{H}-\mathrm{NMR}\left(300 \mathrm{MHz}, \mathrm{DMSO}-\mathrm{d}_{6}\right): \delta[\mathrm{ppm}]=2,40\left(\mathrm{~s}, 3 \mathrm{H},-\mathrm{CH}_{3}\right), 3,73\left(\mathrm{t}, 2 \mathrm{H}, \mathrm{HO}-\mathrm{CH}_{2}\right), 4,10$ (t, $\left.2 \mathrm{H}, \mathrm{O}-\mathrm{CH}_{2}\right), 6,21(\mathrm{~s}, 1 \mathrm{H},-\mathrm{CH}-\mathrm{C}=\mathrm{O}), 6,96\left(\mathrm{~s}, 1 \mathrm{H},-\mathrm{CH}_{\text {aromat: } 6}\right), 6,98\left(\mathrm{~s}, 1 \mathrm{H},-\mathrm{CH}_{\text {aromat:8}}\right)$, $7,70\left(\mathrm{~d}, 1 \mathrm{H},-\mathrm{CH}_{\text {aromat:5}}\right)$.

\section{Synthese von 7-(2-Methacryloyloxyethoxy)-4-methylcumarin (CMA)}

Die Verbindung ist bereits bekannt und die ${ }^{13} \mathrm{C}-\mathrm{NMR}$ Daten sind in der Literatur zu finden. 198

Eine Suspension von 7-(2-Hydroxyethoxy)-4-methylcumarin (5,00 g, 28,4 mmol) und Triethylamin $(4,40 \mathrm{~g}, 43,6 \mathrm{mmol})$ in Chloroform $(100 \mathrm{ml})$ wurde im Eisbad gekühlt. Unter Rühren wurde langsam Methacrylsäurechlorid (5,00 g, 47,8 mmol) zugetropft. Die Lösung wurde für $3 \mathrm{~h}$ bei $0{ }^{\circ} \mathrm{C}$, dann über Nacht bei Raumtemperatur gerührt und mit wässriger $\mathrm{NaOH}$ Lösung $(1 \mathrm{M}, 50 \mathrm{ml})$, Wasser $(2 \times 50 \mathrm{ml})$ und gesättigter $\mathrm{NaCl}$-Lösung gewaschen. Die organische Phase wurde abgetrennt und das Lösungsmittel wurde im Vakuum entfernt. Das Rohprodukt wurde aus Methanol:Ethanol (1:1) umkristallisiert; Ausbeute: 6,95 g, 24,1 mmol (85\%). ${ }^{1} \mathrm{H}-\mathrm{NMR}\left(300 \mathrm{MHz}, \mathrm{CDCl}_{3}\right): \delta[\mathrm{ppm}]=1,95\left(\mathrm{~s}, 3 \mathrm{H}, \mathrm{CH}_{3}-\mathrm{C}=\mathrm{CH}_{2}\right), 2,40(\mathrm{~s}, 3 \mathrm{H}$, C-Ar- $\mathrm{CH}_{3}$ ), 4,28 (t, $2 \mathrm{H}, \mathrm{O}-\mathrm{CH}_{2}$ ), 4,53 (t, $2 \mathrm{H}, \mathrm{O}=\mathrm{C}-\mathrm{O}-\mathrm{CH}_{2}$ ), 5,60 (t, $\left.1 \mathrm{H}, \mathrm{C}=\mathrm{CH}_{2}\right), 6,12-6,15$ (m, $\left.2 \mathrm{H}, \mathrm{C}=\mathrm{CH}_{2},-\mathrm{CH}_{\text {aromat }}-\mathrm{C}=\mathrm{O}\right), 6,83\left(\mathrm{~d}, 1 \mathrm{H},-\mathrm{CH}_{\text {aromat:8}}\right), 6,88\left(\mathrm{dd}, 1 \mathrm{H},-\mathrm{CH}_{\text {aromat:6}}\right), 7,50$ (d, $1 \mathrm{H},-\mathrm{CH}_{\text {aromat:5}}$ ).

\section{Polymersynthesen mit Photovernetzermonomeren}

\section{Allgemeine Polymerisationsvorschrift}

In einem Schraubdeckelglas wurden RAFT-Agens, AIBN und das Monomer im Lösungsmittel gelöst. Die Lösung wurde für mindestens 10 min mit Argon gespült. Währenddessen wurde die Lösung im Eisbad gekühlt, um übermäßiges Verdampfen von Lösungsmittel und flüssigem Monomer zu verhindern. Das Schraubdeckelglas wurde im vortemperierten Heizblock bei 60 oder $70{ }^{\circ} \mathrm{C}$ geschüttelt. Nach der angegeben Zeit wurde das Gefäß entnommen und mit Eis oder mit kaltem Wasser von außen abgekühlt. 


\section{Ergänzende Anmerkungen zur Herstellung von Poly(MMA-co-CMA)}

CMA (5 mol\%) wurde in einer Mischung aus MMA(95 mol\%) und Toluol suspendiert und entgast. Die Gesamtmonomerkonzentration betrug 40 wt\% in Toluol. Im Heizblock entsteht eine homogene Lösung. Nach der Polymerisation wird die viskose Lösung mit Aceton verdünnt, in Methanol gefällt und im Vakuumofen getrocknet.

Tab. 6.1. Reaktionsbedingungen der CMA/MMA-Copolymere mit 5 mol\% CMA.

\begin{tabular}{|c|c|c|c|c|c|}
\hline Probe & Mo:RAFT:I & $t / \mathrm{h}$ & $T /{ }^{\circ} \mathrm{C}$ & $M_{\mathrm{n}} /\left(\mathrm{g} \mathrm{mol}^{-1}\right)$ & 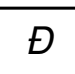 \\
\hline Poly(MMA-co-CM & $50: 1: 0,1$ & 18 & 70 & 6,5 & 1,1 \\
\hline Poly $(\mathrm{MMA}-\mathrm{co}-\mathrm{CMA})_{7 \mathrm{k}}$ & $50: 1: 0,1$ & 18 & 70 & 7,3 & 1,2 \\
\hline Poly(MMA-co-CMA $)_{9 k}$ & $100: 1: 0,2$ & 22 & 60 & 8,7 & 1,2 \\
\hline Poly(MMA-co-CMA) $)_{13 \mathrm{k}}$ & $150: 1: 0,2$ & 22 & 60 & 13 & 1,1 \\
\hline
\end{tabular}

\section{Ergänzende Anmerkungen zur Herstellung von Poly[(MMA-co-CMA)-b-BA]}

Das Poly(MMA-co-CMA) wird als Makro-RAFT-Agens eingesetzt. Als Lösungsmittel wurde Toluol verwendet. Die Konzentration an BA betrug $350 \mathrm{mg} \mathrm{L}^{-1}$. Nach der Polymerisation wurde die viskose Lösung mit Aceton verdünnt, in eiskaltes Methanol gefällt und im Vakuumofen getrocknet.

Tab. 6.2. Reaktionsbedingungen, Molmasse und Dispersität der Poly(MMA-co-CMA)- $b$-Poly(BA) Blockcopolymere.

\begin{tabular}{|c|c|c|c|c|c|}
\hline Probe & Mo:RAFT:I & $T /{ }^{\circ} \mathrm{C}$ & $t / \mathrm{h}$ & $\begin{array}{c}M_{\mathrm{n}} \\
/\left(\mathrm{g} \mathrm{mol}^{-1}\right)\end{array}$ & 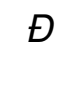 \\
\hline $\mathrm{P}(\mathrm{MMM}-\mathrm{co}-\mathrm{CMA})_{6 \mathrm{k}}-\mathrm{PBA}_{10 \mathrm{k}}$ & $150: 1: 0,1$ & 1 & 70 & 16 & 1,2 \\
\hline $\mathrm{P}(\mathrm{MMM}-\mathrm{co}-\mathrm{CMA})_{6 \mathrm{k}}-\mathrm{PBA}_{19 \mathrm{k}}$ & $150: 1: 0,1$ & 19 & 70 & 25 & 1,3 \\
\hline $\mathrm{P}(\mathrm{MMM}-c o-\mathrm{CMA})_{7 \mathrm{k}}-\mathrm{PB}_{11 \mathrm{k}}$ & $150: 1: 0,1$ & 15 & 70 & 19 & 1,3 \\
\hline $\mathrm{P}(\mathrm{MMM}-c o-\mathrm{CM} A)_{7 \mathrm{k}}-\mathrm{PB}_{12 \mathrm{k}}$ & $150: 1: 0,1$ & 22 & 70 & 20 & 1,3 \\
\hline $\mathrm{P}(\mathrm{MMM}-c o-\mathrm{CMA})_{9 \mathrm{k}}-\mathrm{PB}_{16 \mathrm{k}}$ & $150: 1: 0,2$ & 20 & 60 & 26 & 1,3 \\
\hline $\mathrm{P}(\mathrm{MMM}-\mathrm{co}-\mathrm{CMA})_{9 \mathrm{k}}-\mathrm{PB}_{15 \mathrm{k}}$ & $130: 1: 0.2$ & 20 & 60 & 24 & 1,3 \\
\hline $\mathrm{P}(\mathrm{MMM}-\mathrm{co}-\mathrm{CMA})_{13 \mathrm{k}}-\mathrm{PB}_{16 \mathrm{k}}$ & $140: 1: 0,2$ & 19 & 60 & 29 & 1,3 \\
\hline $\mathrm{P}(\mathrm{MMM}-\mathrm{co}-\mathrm{CMA})_{13 \mathrm{k}}-\mathrm{PB}_{20 \mathrm{k}}$ & $200: 1: 0,2$ & 19 & 60 & 33 & 1,7 \\
\hline
\end{tabular}

\section{Ergänzende Anmerkungen zur Herstellung von Poly(LMA-co-ANMA)}

ANMA wurde zuerst in DMF gelöst. Anschließend wurde die Lösung mit zwei Teilen bzw. der doppelten Menge Butanon und dann mit drei Teilen bzw. der dreifachen Menge LMA verdünnt, dabei kann ein kleiner Teil des gelösten ANMA wieder ausfallen.

Nach der Polymerisation wird das Polymer in Ethanol gefällt und zentrifugiert. Die Lösung wird abdekantiert und das Polymer in wenig THF gelöst und erneut in $\mathrm{MeOH}$ gefällt. Nach 
Zentrifugierung des Polymers und Abdekantieren der Lösung wird der Aufreinigungsschritt bestehend aus Lösen und Fällen wiederholt. Das Polymer wird im Vakuumofen bei $50{ }^{\circ} \mathrm{C}$ getrocknet.

Tab. 6.3. Erhaltene Molmasse, Dispersität und Zusammensetzung der statistischen Copolymere aus ANMA und LMA bzw. BMA. Alle Polymerisationen wurden bei $60^{\circ} \mathrm{C}$ durchgeführt.

\begin{tabular}{|c|c|c|c|c|c|}
\hline Probe & Mo:RAFT:I & Monomer & $t / \mathrm{h}$ & $\begin{array}{c}M_{\mathrm{n}} \\
/\left(\mathrm{g} \mathrm{mol}^{-1}\right)\end{array}$ & $\theta$ \\
\hline $\mathrm{P}\left(\mathrm{LMA}_{0,83}-\right.$ co-ANMA $\left.{ }_{0,17}\right)$ & $300: 1: 0,2$ & $\begin{array}{l}\text { LMA } 83 \% \\
\text { ANMA } 17 \%\end{array}$ & 17 & 48,9 & 1,14 \\
\hline $\mathrm{P}\left(\mathrm{LMA}_{0,92}-\mathrm{co}-\mathrm{ANMA}_{0,08}\right)$ & $300: 1: 0,2$ & $\begin{array}{l}\text { LMA } 91 \% \\
\text { ANMA } 9 \%\end{array}$ & 17 & 48,2 & 1,22 \\
\hline $\mathrm{P}\left(\mathrm{LMA}_{0,95}-\mathrm{co}-\mathrm{ANMA}_{0,05}\right)$ & $300: 1: 0,2$ & $\begin{array}{l}\text { LMA } 95 \% \\
\text { ANMA } 5 \%\end{array}$ & 17 & 51,5 & 1,18 \\
\hline $\mathrm{PLMA}_{21 \mathrm{k}}$ & $100: 1: 0,2$ & LMA & 14 & 20,7 & 1,16 \\
\hline$P\left(\right.$ LMA-co-ANMA $\left.{ }_{21 \mathrm{k}}\right)$ & $100: 1: 0,2$ & $\begin{array}{l}90 \% \text { LMA, } \\
10 \% \text { ANMA }\end{array}$ & 14,5 & 21,2 & 1,20 \\
\hline
\end{tabular}

\section{Ergänzende Anmerkungen zur Herstellung von Poly(BMA-co-ANMA) und Poly(BMA-co-MMA)}

Das ANMA wurde zuerst in DMSO gelöst und die Lösung wurde dann mit dem gleichen Volumen an DMF verdünnt. Im Fall von Poly(BMA-co-MMA) wurde die Polymerisation in Butanon (50 wt\%) durchgeführt. Nach der Polymerisation wurden die Polymere analog zu Poly(LMA-Co-ANMA) aufgearbeitet.

Tab. 6.4. Erhaltene Molmassen, Dispersitäten und Zusammensetzungen der statistischen Copolymere aus BMA und ANMA bzw. MMA. Alle Polymerisationen wurden bei $60^{\circ} \mathrm{C}$ durchgeführt.

\begin{tabular}{|c|c|c|c|c|c|}
\hline Probe & Mo:RAFT:I & Monomer & $t / \mathrm{h}$ & $\begin{array}{c}M_{\mathrm{n}} \\
/\left(\mathrm{g} \mathrm{mol}^{-1}\right)\end{array}$ & $\theta$ \\
\hline $\mathrm{P}\left(\mathrm{BMA}-\mathrm{co}-\mathrm{MMA}_{10 \mathrm{k}}\right)$ & $100: 1: 0,2$ & $\begin{array}{l}20 \% \text { BMA, } \\
80 \% \text { MMA }\end{array}$ & 13,5 & 10,1 & 1,18 \\
\hline $\mathrm{P}\left(\mathrm{BMA}_{0,9}-\mathrm{Co}-\mathrm{ANMA}_{0,1}\right)$ & $300: 1: 0,1$ & $\begin{array}{l}\text { BMA } 90 \% \\
\text { ANMA } 10 \%\end{array}$ & 24 & 36,2 & 1,16 \\
\hline
\end{tabular}

\section{Ergänzende Anmerkungen zur Herstellung der ANMA-enthaltenden Blockcopolymere}

Der erste Block wird als Makro-RAFT-Agens eingesetzt.

Wurde ANMA als Monomer im zweiten Block eingesetzt, dann wurde Butanon:DMF 2:1 als Lösungsmittelgemisch verwendet und ANMA wurde analog zu Poly(LMA-co-ANMA) zuerst 
nur in DMF gelöst. Wurde ANMA nicht als Monomer im zweiten Block eingesetzt, dann wird Butanon als Lösungsmittel eingesetzt. Makro-RAFT-Agens und Monomer werden zusammen (50 wt\%) im Lösungsmittel(-gemisch) gelöst. Die weitere Vorgehensweise ist analog zu Poly(LMA-co-ANMA). Die Details der Synthesen der Blockcopolymere finden sich in den Tabellen 5.6 und 5.5 $\mathrm{im}$ Abschnitt5.3.2. 


\section{Literaturverzeichnis}

[1] Wöhrle, D. Chem. Unserer Zeit 2018, 52, 2-16.

[2] MacDermott, C. P.; Shenoy, A. V. Selecting thermoplastics for engineering applications, 2. Auflage; CRC Press: New York, 1997.

[3] Moad, G.; Rizzardo, E.; Thang, S. H. Austr. J. Chem. 2005, 58, 379-410.

[4] Matyjaszewski, K.; Xia, J. Chem. Rev. 2001, 101, 2921-2990.

[5] Spontak, R. J.; Patel, N. P. Curr. Opin. Colloid Interface Sci. 2000, 5, 333-340.

[6] Hentschel, J.; Kushner, A. M.; Ziller, J.; Guan, Z. Angew. Chem. Int. Ed. 2012, 124, 10713-10717.

[7] Amin, S.; Amin, M. Rev. Adv. Mater. Sci. 2011, 29, 15-30.

[8] Schacher, F. H.; Rupar, P. A.; Manners, I. Angew. Chem. Int. Ed. 2012, 51, 7898-7921.

[9] Nielsen, L. E. J. Macromol. Sci. Polymer Rev. 1969, 3, 69-103.

[10] Krejsa, M.; Koenig, J. Rubber Chem. Technol. 1993, 66, 376-410.

[11] Fox, T. G.; Flory, P. J. J. Polym. Sci. 1954, 14, 315-319.

[12] Beevers, R.; White, E. Trans. Faraday Soc. 1960, 56, 744-752.

[13] Nunes, R. W.; Martin, J. R.; Johnson, J. F. Polym. Eng. Sci. 1982, 22, 205-228.

[14] Kostanski, L. K.; Keller, D. M.; Hamielec, A. E. J. Biochem. Biophys. Methods 2004, 58, 159-186.

[15] Fenn, J. B.; Mann, M.; Meng, C. K.; Wong, S. F.; Whitehouse, C. M. Science 1989, 246, 64-71.

[16] Tanaka, K.; Waki, H.; Ido, Y.; Akita, S.; Yoshida, Y.; Yoshida, T.; Matsuo, T. Rapid Commun. Mass Spectrom. 1988, 2, 151-153.

[17] Karas, M.; Bachmann, D.; Hillenkamp, F. Anal. Chem. 1985, 57, 2935-2939.

[18] Hanton, S. Chem. Rev. 2001, 101, 527-570.

[19] Peacock, P. M.; McEwen, C. N. Anal. Chem. 2004, 76, 3417-3428.

[20] Alhazmi, A. M.; Giguère, M.-S.; Dubé, M. A.; Mayer, P. M. Eur. J. Mass Spectrom. 2006, 12, 301-310.

[21] Huijser, S.; Staal, B. B.; Huang, J.; Duchateau, R.; Koning, C. E. Angew. Chem. 2006, 118, 4210-4214.

[22] Janiak, C.; Lange, K. C.; Marquardt, P.; Krüger, R.-P.; Hanselmann, R. Macromol. Chem. Phys. 2002, 203, 129-138.

[23] Yalcin, T.; Wallace, W. E.; Guttman, C. M.; Li, L. Anal. Chem. 2002, 74, 4750-4756.

[24] Briquel, R.; Mazzolini, J.; Le Bris, T.; Boyron, O.; Boisson, F.; Delolme, F.; D’Agosto, F.; Boisson, C.; Spitz, R. Angew. Chem. Int. Ed. 2008, 47, 9311-9313.

[25] Gibson, V. C. Science 2006, 312, 703-704.

[26] German, I.; Kelhifi, W.; Norsic, S.; Boisson, C.; D’Agosto, F. Angew. Chem. 2013, 125, 3522-3525.

[27] Georges, M. K.; Veregin, R. P.; Kazmaier, P. M.; Hamer, G. K. Macromolecules 1993, 26, 2987-2988.

[28] Grubbs, R. B. Polymer Reviews 2011, 51, 104-137.

[29] Matyjaszewski, K. Macromolecules 2012, 45, 4015-4039. 
[30] Thang, S. H.; Rizzardo, E.; Moad, G. Macromolecules 1998, 31, 5559-5562.

[31] Bicciocchi, E.; Chong, Y. K.; Giorgini, L.; Moad, G.; Rizzardo, E.; Thang, S. H. Macromol. Chem. Phys. 2010, 211, 529-538.

[32] Moad, G. Macromol. Chem. Phys. 2014, 215, 9-26.

[33] Seymour, R. B. Advances in Polyolefins; Springer, 1987; pp 3-14.

[34] Mazzolini, J.; Espinosa, E.; D’Agosto, F.; Boisson, C. Polym. Chem. 2010, 1, 793-800.

[35] Zhang, Y.; Li, H.; Dong, J.-Y.; Hu, Y. Polym. Chem. 2014, 5, 105-115.

[36] Kaneyoshi, H.; Inoue, Y.; Matyjaszewski, K. Macromolecules 2005, 38, 5425-5435.

[37] Maher, S.; Jjunju, F. P.; Taylor, S. Rev. Mod. Phys. 2015, 87, 113.

[38] Bruins, A. Mass Spectrom. Rev. 1991, 10, 53-77.

[39] Gross, J. H. Mass spectrometry: a textbook, 3rd ed.; Springer Science \& Business Media, 2017.

[40] Dawson, P. Mass Spectrom. Rev. 1986, 5, 1-37.

[41] Douglas, D. J.; Frank, A. J.; Mao, D. Mass Spectrom. Rev. 2005, 24, 1-29.

[42] Paul, W.; Steinwedel, H. Z. Naturforsch. A 1953, 8, 448-450.

[43] March, R. E. J. Mass Spectrom. 1997, 32, 351-369.

[44] Hu, Q.; Noll, R. J.; Li, H.; Makarov, A.; Hardman, M.; Graham Cooks, R. J. Mass Spectrom. 2005, 40, 430-443.

[45] Makarov, A. Anal. Chem. 2000, 72, 1156-1162.

[46] Comisarow, M. B.; Marshall, A. G. Chem. Phys. Lett. 1974, 25, 282-283.

[47] Marshall, A. G.; Hendrickson, C. L.; Jackson, G. S. Mass Spectrom. Rev 1998, 17, 1-35.

[48] Jakubowski, N.; Moens, L.; Vanhaecke, F. Spectrochim. Acta B 1998, 53, 1739-1763.

[49] Wollnik, H. Mass Spectrom. Rev. 1993, 12, 89-114.

[50] Cotter, R. J. Anal. Chem. 1999, 71, 445A-451A.

[51] Radionova, A.; Filippov, I.; Derrick, P. J. Mass Spectrom. Rev. 2016, 35, 738-757.

[52] Boesl, U. Mass Spectrom. Rev. 2017, 36, 86-109.

[53] Märk, T. D.; Dunn, G. H. Electron impact ionization; Springer-Verlag, 1985.

[54] Kind, T.; Fiehn, O. Bioanal Rev 2010, 2, 23-60.

[55] Awad, H.; Khamis, M. M.; El-Aneed, A. Appl. Spectrosc. Rev. 2015, 50, 158-175.

[56] Fenn, J. B. Angew. Chem. Int. Ed. 2003, 42, 3871-3894.

[57] Tanaka, K. Angew. Chem. Int. Ed. 2003, 42, 3860-3870.

[58] El-Aneed, A.; Cohen, A.; Banoub, J. Appl. Spectrosc. Rev. 2009, 44, 210-230.

[59] Wilm, M. Mol. Cell. Proteomics 2011, mcp-R111.

[60] Konermann, L.; Ahadi, E.; Rodriguez, A. D.; Vahidi, S. Anal. Chem. 2013, 85, 2-9.

[61] Banerjee, S.; Mazumdar, S. Int. J. Anal. Chem. 2012, 2012.

[62] Konermann, L.; Rodriguez, A. D.; Liu, J. Anal. Chem. 2012, 84, 6798-6804.

[63] Ahadi, E.; Konermann, L. J. Phys. Chem. B 2011, 116, 104-112.

[64] Wong, S.; Meng, C.; Fenn, J. J. Phys. Chem. 1988, 92, 546-550.

[65] Karas, M.; Bahr, U.; Gießmann, U. Mass Spectrom. Rev. 1991, 10, 335-357.

[66] Hillenkamp, F.; Karas, M.; Beavis, R. C.; Chait, B. T. Anal. Chem. 1991, 63, 1193A$1203 \mathrm{~A}$.

[67] Zenobi, R.; Knochenmuss, R. Mass Spectrom. Rev. 1998, 17, 337-366. 
[68] Schriemer, D. C.; Li, L. Anal. Chem. 1996, 68, 2721-2725.

[69] Karas, M.; Glückmann, M.; Schäfer, J. J. Mass Spectrom. 2000, 35, 1-12.

[70] Nielen, M. W. Mass Spectrom. Rev. 1999, 18, 309-344.

[71] Schriemer, D. C.; Li, L. Anal. Chem. 1997, 69, 4176-4183.

[72] Strupat, K.; Karas, M.; Hillenkamp, F. Int. J. Mass Spectrom. Ion Processes 1991, 111, 89-102.

[73] Westman, A.; Huth-Fehre, T.; Demirev, P.; Sundqvist, B. J. Mass Spectrom. 1995, 30, 206-211.

[74] Chan, P.; Dominic Chan, T.-W. Rapid Commun. Mass Spectrom. 2000, 14, 18411847.

[75] Brown, R. S.; Lennon, J. J. Anal. Chem. 1995, 67, 1998-2003.

[76] Wetzel, S. J.; Guttman, C. M.; Girard, J. E. Int. J. Mass Spectrom. 2004, 238, 215-225.

[77] Wetzel, S. J.; Guttman, C. M.; Girard, J. E. Polym. Mater. Sci. Eng. 2003, 88, 74-75.

[78] Jaber, A. J.; Kaufman, J.; Liyanage, R.; Akhmetova, E.; Marney, S.; Wilkins, C. L. J. Am. Soc. Mass Spectrom. Chem. 2005, 16, 1772-1780.

[79] Signor, L.; Erba, E. B. J. Vis. Exp. 2013,

[80] Römpp, A.; Spengler, B. Histochem. Cell Biol. 2013, 139, 759-783.

[81] Trimpin, S.; Grimsdale, A. C.; Räder, H. J.; Müllen, K. Anal. Chem. 2002, 74, 37773782.

[82] Trimpin, S.; Keune, S.; Räder, H. J.; Müllen, K. J. Am. Soc. Mass Spectrom. Chem. 2006, 17, 661-671.

[83] Zhang, Z.; Marshall, A. G. J. Am. Soc. Mass Spectrom. Chem. 1998, 9, 225-233.

[84] Wang, Y.; Schubert, M.; Ingendoh, A.; Franzen, J. Rapid Commun. Mass Spectrom. 2000, 14, 12-17.

[85] Axelsson, J.; Scrivener, E.; Haddleton, D. M.; Derrick, P. J. Macromolecules 1996, 29, 8875-8882.

[86] Scalf, M.; Westphall, M. S.; Krause, J.; Kaufman, S. L.; Smith, L. M. Science 1999, 283, 194-197.

[87] Scalf, M.; Westphall, M. S.; Smith, L. M. Anal. Chem. 2000, 72, 52-60.

[88] Stutzman, J. R.; Crowe, M. C.; Alexander IV, J. N.; Bell, B. M.; Dunkle, M. N. Anal. Chem. 2016, 88, 4130-4139.

[89] Ebeling, D. D.; Westphall, M. S.; Scalf, M.; Smith, L. M. Anal. Chem. 2000, 72, 51585161.

[90] Ebeling, D. D.; Westphall, M. S.; Scalf, M.; Smith, L. M. Rapid Commun. Mass Spectrom. 2001, 15, 401-405.

[91] Robb, D. B.; Brown, J. M.; Morris, M.; Blades, M. W. Anal. Chem. 2014, 86, 96449652.

[92] Stephenson, J. L.; McLuckey, S. A. J. Am. Soc. Mass Spectrom. Chem. 1998, 9, 957965.

[93] Lennon, J. D.; Cole, S. P.; Glish, G. L. Anal. Chem. 2006, 78, 8472-8476.

[94] Huang, L.; Gough, P. C.; DeFelippis, M. R. Anal. Chem. 2008, 81, 567-577.

[95] Nasioudis, A.; Joyce, W. F.; Velde, J. W. v.; Heeren, R. M.; Brink, O. F. v. d. Anal. Chem. 2010, 82, 5735-5742.

[96] Lopez, R. G.; Boisson, C.; D’Agosto, F.; Spitz, R.; Boisson, F.; Bertin, D.; Tordo, P. 
Macromolecules 2004, 37, 3540-3542.

[97] Lin-Gibson, S.; Brunner, L.; VanderHart, D. L.; Bauer, B. J.; Fanconi, B.; Guttman, C. M.; Wallace, W. E. Macromolecules 2002, 35, 7149-7156.

[98] Chen, R.; Yalcin, T.; Wallace, W. E.; Guttman, C. M.; Li, L. J. Am. Soc. Mass Spectrom. Chem. 2001, 12, 1186-1192.

[99] Moscato, B.; Landis, C. Chem. Commun. 2008, 5785-5787.

[100] Hinderling, C.; Chen, P. Int. J. Mass Spectrom. 2000, 195, 377-383.

[101] di Lena, F.; Quintanilla, E.; Chen, P. Chem. Commun. 2005, 5757-5759.

[102] Bauer, B. J.; Wallace, W. E.; Fanconi, B. M.; Guttman, C. M. Polymer 2001, 42, 0994909953.

[103] Wallace, W. E.; Blair, W. R. Int. J. Mass Spectrom. 2007, 263, 82-87.

[104] Bergbreiter, D. E.; Blanton, J. J. Org. Chem. 1985, 50, 5828-5833.

[105] Grinberg, S.; Kas' yanov, V.; Srinivas, B. React. Funct. Polym. 1997, 34, 53-63.

[106] Bergbreiter, D. E. Chem. Rev. 2002, 102, 3345-3384.

[107] Lodge, T. P. Macromol. Chem. Phys. 2003, 204, 265-273.

[108] Segalman, R. A. Mater. Sci. Eng. R Rep. 2005, 48, 191-226.

[109] Corredor, C. C.; Huang, Z.-L.; Belfield, K. D.; Morales, A. R.; Bondar, M. V. Chem. Mat. 2007, 19, 5165-5173.

[110] Sriprom, W.; Néel, M.; Gabbutt, C. D.; Heron, B. M.; Perrier, S. J. Mater. Chem. 2007, 17, 1885-1893.

[111] Barner-Kowollik, C.; Perrier, S. J. Polym. Sci., Part A: Polym. Chem. 2008, 46, 57155723.

[112] Park, C.; Yoon, J.; Thomas, E. L. Polymer 2003, 44, 6725-6760.

[113] Matsen, M. W.; Bates, F. S. Macromolecules 1996, 29, 1091-1098.

[114] Matsen, M. W.; Schick, M. Macromolecules 1994, 27, 7157-7163.

[115] Bates, F. S.; Fredrickson, G. Phys. Today 1999, 52, 32-38.

[116] Mai, Y.; Eisenberg, A. Chem. Soc. Rev. 2012, 41, 5969-5985.

[117] Bates, F. S.; Fredrickson, G. H. Annu. Rev. Phys. Chem. 1990, 41, 525-557.

[118] Schwarzl, F. R. Polymermechanik: Struktur und mechanisches Verhalten von Polymeren; Springer-Verlag, 2013.

[119] Tieke, B. Makromolekulare Chemie: Eine Einführung, 3rd ed.; John Wiley \& Sons, 2014.

[120] Davis, J. R. Tensile testing; ASM international, 2004.

[121] Martin, J. R.; Johnson, J. F.; Cooper, A. R. J. Macromol. Sci. Rev. Macromol. Chem. 1972, 8, 57-199.

[122] Bi, L.-K.; Fetters, L. J. Macromolecules 1976, 9, 732-742.

[123] Kruželák, J.; Sỳkora, R.; Hudec, I. Chem. Pap. 2016, 70, 1533-1555.

[124] Tillet, G.; Boutevin, B.; Ameduri, B. Prog. Polym. Sci. 2011, 36, 191-217.

[125] Coleman, M. M.; Shelton, J. R.; Koenig, J. L. Ind. Eng. Chem. Prod. Res. Dev. 1974, 13, 154-166.

[126] Shi, D.; Matsusaki, M.; Kaneko, T.; Akashi, M. Macromolecules 2008, 41, 8167-8172.

[127] Coqueret, X. Macromol. Chem. Phys. 1999, 200, 1567-1579.

[128] Coleman, M. M.; Hu, Y.; Sobkowiak, M.; Painter, P. C. J. Polym. Sci., Part B: Polym. Phys. 1998, 36, 1579-1590. 
[129] Seiffert, S.; Oppermann, W.; Saalwächter, K. Polymer 2007, 48, 5599-5611.

[130] Finter, J.; Widmer, E.; Zweifel, H. Angew. Makromol. Chem. 1984, 128, 71-97.

[131] Finter, J.; Haniotis, Z.; Lohse, F.; Meier, K.; Zweifel, H. Angew. Makromol. Chem. 1985, 133, 147-170.

[132] Chen, Y.; Geh, J.-L. Polymer 1996, 37, 4481-4486.

[133] Chujo, Y.; Sada, K.; Saegusa, T. Macromolecules 1990, 23, 2693-2697.

[134] Ravve, A. Light-associated reactions of synthetic polymers.; Springer, 2006.

[135] Trenor, S. R.; Shultz, A. R.; Love, B. J.; Long, T. E. Chem. Rev. 2004, 104, 3059-3078.

[136] Chen, Y.; Wu, J.-D. J. Polym. Sci., Part A: Polym. Chem. 1994, 32, 1867-1875.

[137] Matsui, J.; Ochi, Y.; Tamaki, K. Chem. Lett. 2005, 35, 80-81.

[138] Shi, Y.; Cardoso, R. M.; Van Nostrum, C. F.; Hennink, W. E. Polym. Chem. 2015, 6, 2048-2053.

[139] Van Damme, J.; Du Prez, F. Prog. Polym. Sci. 2018,

[140] Yokoe, M.; Yamauchi, K.; Long, T. E. J. Polym. Sci., Part A: Polym. Chem. 2016, 54, 2302-2311.

[141] Van Damme, J.; Vlaminck, L.; Van Assche, G.; Van Mele, B.; van den Berg, O.; Du Prez, F. Tetrahedron 2016, 72, 4303-4311.

[142] Simionescu, C. I.; Grigoras, M.; Bărboiu, V. J. Polym. Sci.: Polym. Chem. Ed. 1985, 23, 2089-2098.

[143] Oh, J. K.; Wu, J.; Winnik, M. A.; Craun, G. P.; Rademacher, J.; Farwaha, R. J. Polym. Sci., Part A: Polym. Chem. 2002, 40, 1594-1607.

[144] Liu, R.; Winnik, M. A.; Di Stefano, F.; Vanketessan, J. J. Polym. Sci., Part A: Polym. Chem. 2001, 39, 1495-1504.

[145] Zheng, Y.; Micic, M.; Mello, S. V.; Mabrouki, M.; Andreopoulos, F. M.; Konka, V.; Pham, S. M.; Leblanc, R. M. Macromolecules 2002, 35, 5228-5234.

[146] Froimowicz, P.; Frey, H.; Landfester, K. Macromol. Rapid Commun. 2011, 32, 468473.

[147] Chung, C.-M.; Roh, Y.-S.; Cho, S.-Y.; Kim, J.-G. Chem. Mat. 2004, 16, 3982-3984.

[148] Habault, D.; Zhang, H.; Zhao, Y. Chem. Soc. Rev. 2013, 42, 7244-7256.

[149] Fiore, G. L.; Rowan, S. J.; Weder, C. Chem. Soc. Rev. 2013, 42, 7278-7288.

[150] Staudt, B. H.; Wagner, J.; Vana, P. Macromolecules 2018, 51, 8469-8476.

[151] Baier, M. C.; Zuideveld, M. A.; Mecking, S. Angew. Chem. Int. Ed. 2014, 53, 97229744.

[152] Sauter, D. W.; Taoufik, M.; Boisson, C. Polymers 2017, 9, 185.

[153] Zhang, L.; Cool, L. R.; Wesdemiotis, C.; Weiss, R.; Cavicchi, K. A. Polym. Chem. 2014, $5,1180-1190$.

[154] Zhang, L.; Tang, Q.; Weiss, R.; Cavicchi, K. A. Polym. Chem. 2014, 5, 5492-5500.

[155] Henderson, M. A.; Mclndoe, J. S. Chem. Commun. 2006, 2872-2874.

[156] Kebarle, P. J. Mass Spectrom. 2000, 35, 804-817.

[157] Mora, J. F. d. I.; Van Berkel, G. J.; Enke, C. G.; Cole, R. B.; Martinez-Sanchez, M.; Fenn, J. B. J. Mass Spectrom. 2000, 35, 939-952.

[158] Gruendling, T.; Junkers, T.; Guilhaus, M.; Barner-Kowollik, C. Macromol. Chem. Phys. 2010, 211, 520-528.

[159] Appendini, P.; Hotchkiss, J. H. Innov. Food Sci. Emerg. Technol. 2002, 3, 113-126. 
[160] Rahman, M.; Brazel, C. S. Prog. Polym. Sci. 2004, 29, 1223-1248.

[161] Liu, R.; Li, Z. Y.; Wang, W. J.; Yuan, D.; Meng, C. F.; Wu, Q.; Zhu, F. M. J. Appl. Polym. Sci. 2013, 129, 2216-2223.

[162] Sinturel, C.; Bates, F. S.; Hillmyer, M. A. ACS Macro Lett. 2015, 4, 1044-1050.

[163] Peacock, P. M.; McEwen, C. N. Anal. Chem. 2006, 78, 3957-3964.

[164] Feldermann, A.; Toy, A. A.; Davis, T. P.; Stenzel, M. H.; Barner-Kowollik, C. Polymer 2005, 46, 8448-8457.

[165] Ah Toy, A.; Vana, P.; Davis, T. P.; Barner-Kowollik, C. Macromolecules 2004, 37, 744751.

[166] Lovestead, T. M.; Hart-Smith, G.; Davis, T. P.; Stenzel, M. H.; Barner-Kowollik, C. Macromolecules 2007, 40, 4142-4153.

[167] Ponnusamy, K.; Babu, R. P.; Dhamodharan, R. J. Polym. Sci., Part A: Polym. Chem. 2013, 51, 1066-1078.

[168] Chirowodza, H.; Weber, W.; Hartmann, P.; Pasch, H. Surface-Initiated Reversible Addition Fragmentation Chain Transfer (RAFT) Polymerization of Styrene from Laponite Clay Surfaces. Macromolecular Symposia. 2012; pp 135-145.

[169] Moad, G.; Rizzardo, E.; Thang, S. H. Austr. J. Chem. 2012, 65, 985-1076.

[170] Moad, G.; Rizzardo, E.; Thang, S. H. Aust. J. Chem. 2006, 59, 669-692.

[171] Rotzoll, R.; Nguyen, D. H.; Vana, P. 2009, 275, 1-12.

[172] Ladavière, C.; Dörr, N.; Claverie, J. P. Macromolecules 2001, 34, 5370-5372.

[173] Llauro, M.-F.; Loiseau, J.; Boisson, F.; Delolme, F.; Ladavière, C.; Claverie, J. J. Polym. Sci., Part A: Polym. Chem. 2004, 42, 5439-5462.

[174] Gruendling, T.; Hart-Smith, G.; Davis, T. P.; Stenzel, M. H.; Barner-Kowollik, C. Macromolecules 2008, 41, 1966-1971.

[175] Ladaviere, C.; Lacroix-Desmazes, P.; Delolme, F. Macromolecules 2008, 42, 70-84.

[176] Strube, O. I.; Nothdurft, L.; Drache, M.; Schmidt-Naake, G. Macromol. Chem. Phys. 2011, 212, 574-582.

[177] Deery, M. J.; Jennings, K. R.; Jasieczek, C. B.; Haddleton, D. M.; Jackson, A. T.; Yates, H. T.; Scrivens, J. H. Rapid Commun. Mass Spectrom. 1997, 11, 57-62.

[178] Mautjana, N. A.; Looi, D. W.; Eyler, J. R.; Brajter-Toth, A. Electroanalysis 2010, 22, 79-89.

[179] Oberacher, H.; Pitterl, F.; Erb, R.; Plattner, S. Mass Spectrom. Rev. 2015, 34, 64-92.

[180] Peintler-Krivan, E.; Van Berkel, G. J.; Kertesz, V. Rapid Commun. Mass Spectrom. 2010, 24, 1327-1334.

[181] Gierczak, T.; Gilles, M. K.; Bauerle, S.; Ravishankara, A. J. Phys. Chem. A 2003, 107, 5014-5020.

[182] Talukdar, R. K.; Gierczak, T.; McCabe, D. C.; Ravishankara, A. J. Phys. Chem. A 2003, 107, 5021-5032.

[183] Jaquiss, M.; Roberts, J.; Szwarc, M. J. Am. Chem. Soc. 1952, 74, 6005-6007.

[184] Smith, R. D.; Loo, J. A.; Loo, R. R. O.; Busman, M.; Udseth, H. R. Mass Spectrom. Rev. 1991, 10, 359-452.

[185] Karger, B. L.; Vouros, P. J. Chrom. A 1985, 323, 13-32.

[186] Cole, R. B. Electrospray and MALDI mass spectrometry: fundamentals, instrumentation, practicalities, and biological applications; John Wiley \& Sons, 2010. 
[187] Thomson, B. A. J. Am. Soc. Mass Spectrom. Chem. 1997, 8, 1053-1058.

[188] Pashynska, V.; Kosevich, M.; Heuvel, H. V. d.; Claeys, M. Rapid Commun. Mass Spectrom. 2006, 20, 755-763.

[189] Van Dongen, W.; Van Wijk, J.; Green, B.; Heerma, W.; Haverkamp, J. Rapid Commun. Mass Spectrom. 1999, 13, 1712-1716.

[190] Rogniaux, H.; Van Dorsselaer, A.; Barth, P.; Biellmann, J.; Barbanton, J.; Van Zandt, M.; Chevrier, B.; Howard, E.; Mitschler, A.; Potier, N. J. Am. Soc. Mass Spectrom. Chem. 1999, 10, 635-647.

[191] Smith, R. D.; Barinaga, C. J. Rapid Commun. Mass Spectrom. 1990, 4, 54-57.

[192] Gabelica, V.; Pauw, E. D. Mass Spectrom. Rev. 2005, 24, 566-587.

[193] Ran, R.; Yu, Y.; Wan, T. J. Appl. Polym. Sci. 2007, 105, 398-404.

[194] Amamoto, Y.; Kamada, J.; Otsuka, H.; Takahara, A.; Matyjaszewski, K. Angew. Chem. Int. Ed. 2011, 50, 1660-1663.

[195] Haven, J.; Junkers, T. Polymers 2018, 10, 1228.

[196] Buback, M.; Vana, P. Macromol. Rapid Commun. 2006, 27, 1299-1305.

[197] Meiser, W.; Buback, M.; Ries, O.; Ducho, C.; Sidoruk, A. Macromol. Chem. Phys. 2013, 214, 924-933.

[198] Sinkel, C.; Greiner, A.; Agarwal, S. Macromol. Chem. Phys. 2010, 211, 1857-1867.

[199] Tietz, K. Mikrophasenseparation von photo-vernetzbaren Blockcopolymeren in dünnen Filmen. Ph.D. thesis, Georg-August-Universität Göttingen, 2015.

[200] Puskas, J.; Antony, P.; El Fray, M.; Altstädt, V. Eur. Polym. J. 2003, 39, 2041-2049.

[201] Binnig, G.; Quate, C. F.; Gerber, C. Phys. Rev. Lett. 1986, 56, 930.

[202] Giessibl, F. J. Rev. Mod. Phys. 2003, 75, 949.

[203] Sriprom, W.; James, M.; Perrier, S.; Neto, C. Macromolecules 2009, 42, 3138-3146.

[204] Sakamoto, H.; Anase, T.; Osuga, H.; Kimura, K. React. Funct. Polym. 2011, 71, 569573.

[205] Floudas, G.; Placke, P.; Stepanek, P.; Brown, W.; Fytas, G.; Ngai, K. Macromolecules 1995, 28, 6799-6807.

[206] Rogers, S.; Mandelkern, L. J. Phys. Chem. 1957, 61, 985-991.

[207] Fleischhaker, F.; Haehnel, A. P.; Misske, A. M.; Blanchot, M.; Haremza, S.; BarnerKowollik, C. Macromol. Chem. Phys. 2014, 215, 1192-1200.

[208] Steinhoff, J. E. Mechanische Eigenschaften von Polymer-Silica-Nanokompositen. M.Sc. thesis, Georg-August-Universität Göttingen, 2016.

[209] Bang, J.; Jeong, U.; Ryu, D. Y.; Russell, T. P.; Hawker, C. J. Adv. Mater. 2009, 21, 4769-4792.

[210] Krausch, G. Mater. Sci. Eng. R Rep. 1995, 14, v-94.

[211] Darling, S. Prog. Polym. Sci. 2007, 32, 1152-1204.

[212] Derjaguin, B. V.; Muller, V. M.; Toporov, Y. P. J. Colloid Interface Sci. 1975, 53, 314326.

[213] Muller, V.; Yushchenko, V.; Derjaguin, B. J. Colloid Interface Sci. 1980, 77, 91-101.

[214] Kaemmer, S. B. Introduction to Bruker's ScanAsyst and PeakForce Tapping AFM Technology. Bruker: Bruker application note AN133, 2011.

[215] Bowen, E.; Tanner, D. Trans. Faraday Soc. 1955, 51, 475-481.

[216] Fox, M. A.; Olive, S. Science 1979, 205, 582-583. 
[217] Yamamoto, T.; Yagyu, S.; Tezuka, Y. J. Am. Chem. Soc. 2016, 138, 3904-3911.

[218] Menard, K. P. Dynamic mechanical analysis: a practical introduction; CRC press, 2008.

[219] Arua, U. N.; Blum, F. D. J. Polym. Sci., Part B: Polym. Phys. 2018, 56, 89-96.

[220] Abiad, M. G.; Campanella, O. H.; Carvajal, M. T. Pharmaceutics 2010, 2, 78-90.

[221] Use of Material Pockets for Mechanical Analysis of Powders. PerkinElmer: PerkinElmer Application Note, 2011.

[222] Fan, L.; Zhao, D.; Bian, C.; Wang, Y.; Liu, G. Polym. Bull. 2011, 67, 1311.

[223] Fernández-García, M.; Cuervo-Rodriguez, R.; Madruga, E. J. Polym. Sci., Part B: Polym. Phys. 1999, 37, 2512-2520.

[224] Gaur, U.; Wunderlich, B. Macromolecules 1980, 13, 1618-1625.

[225] Gracia-Fernández, C.; Gómez-Barreiro, S.; López-Beceiro, J.; Saavedra, J. T.; Naya, S.; Artiaga, R. Polym. Test. 2010, 29, 1002-1006.

[226] Achorn, P. J.; Ferrillo, R. G. J. Appl. Polym. Sci. 1994, 54, 2033-2043.

[227] Zhao, F.; Bi, W.; Zhao, S. J. Macromol. Sci., Part B: Phys 2011, 50, 1460-1469.

[228] Deschamps, A. A.; Grijpma, D. W.; Feijen, J. Polymer 2001, 42, 9335-9345.

[229] Bellin, I.; Kelch, S.; Langer, R.; Lendlein, A. Proc. Natl. Acad. Sci. U.S.A. 2006, 103, 18043-18047.

[230] Zhao, J.; Chen, M.; Wang, X.; Zhao, X.; Wang, Z.; Dang, Z.-M.; Ma, L.; Hu, G.-H.; Chen, F. ACS Appl. Mater. Interfaces 2013, 5, 5550-5556.

[231] Xie, T. Polymer 2011, 52, 4985-5000.

[232] Wei, M.; Zhan, M.; Yu, D.; Xie, H.; He, M.; Yang, K.; Wang, Y. ACS Appl. Mater. Interfaces 2015, 7, 2585-2596.

[233] Buback, M.; Feldermann, A.; Barner-Kowollik, C.; Lacík, I. Macromolecules 2001, 34, 5439-5448.

[234] Hutchinson, R.; Beuermann, S.; Paquet, D.; McMinn, J. Macromolecules 1997, 30 , 3490-3493. 


\section{Anhang A: Abbildungen}

Verweise auf Abbildungen in diesem Anhang werden im Text mit dem Präfix A gekennzeichnet.

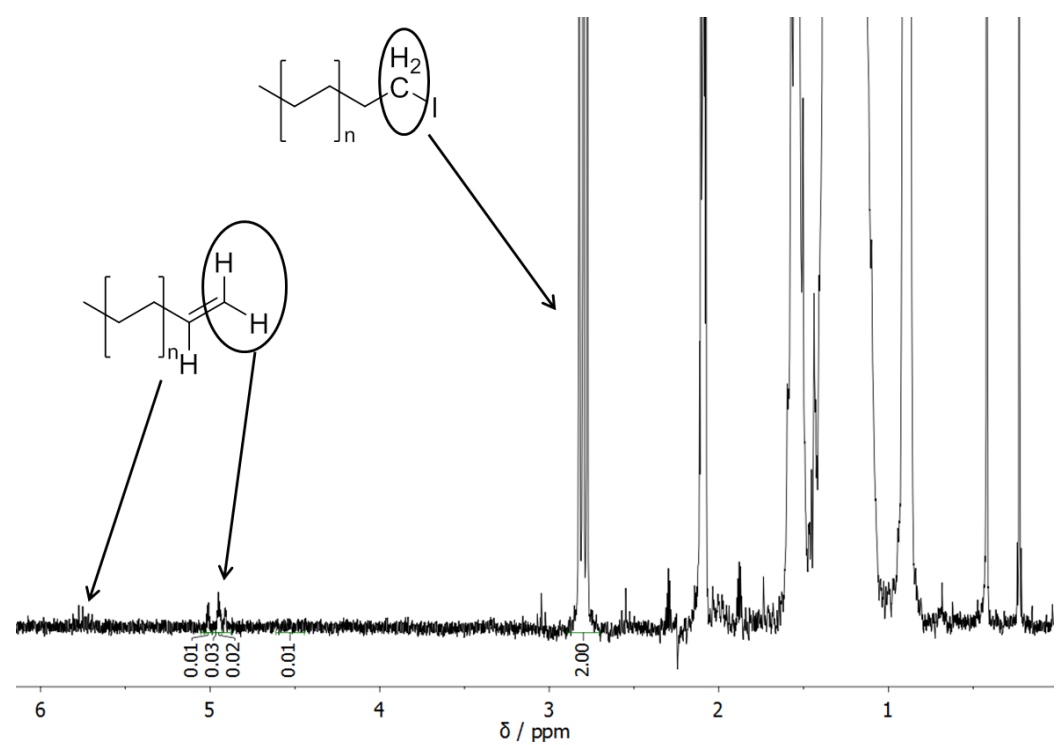

A1. ${ }^{1} \mathrm{H}-\mathrm{NMR}-$ Sektrum von PE- $\mathrm{N}^{+}$. Terminale Vinylendgruppen sind praktisch nicht vorhanden.

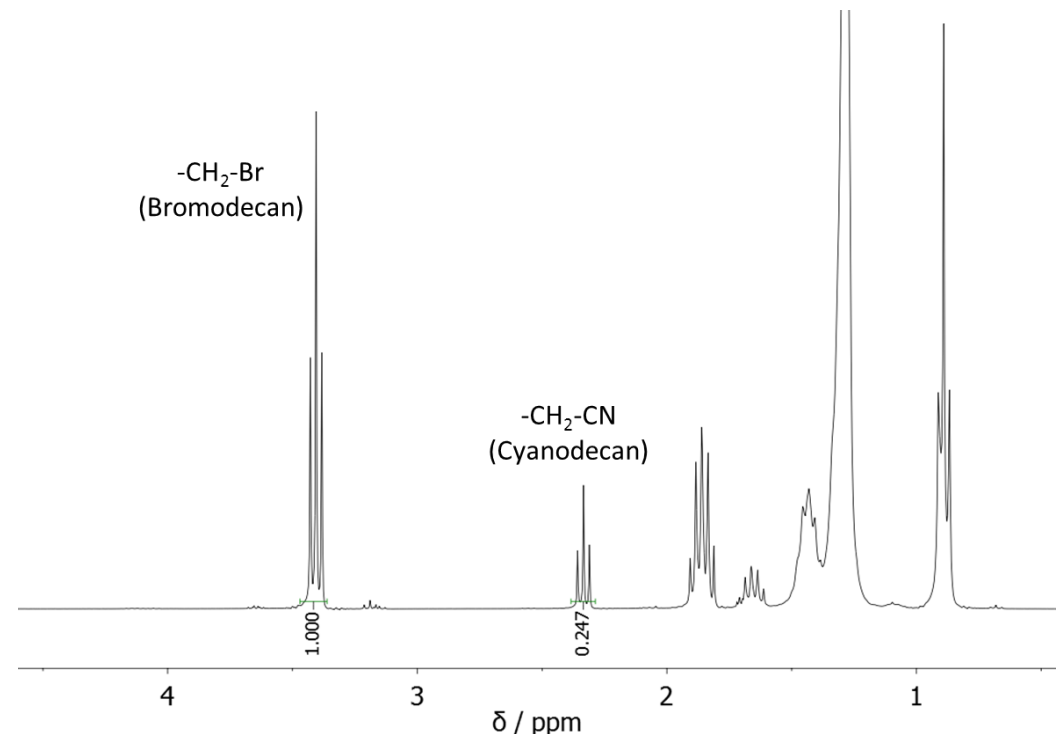

A2. ${ }^{1} \mathrm{H}-\mathrm{NMR}$-Sektrum der Umsetzung von 1-Bromodecan mit Natriumcyanid und PE-N ${ }^{+}$als PTK nach vier Stunden Reaktionszeit. 

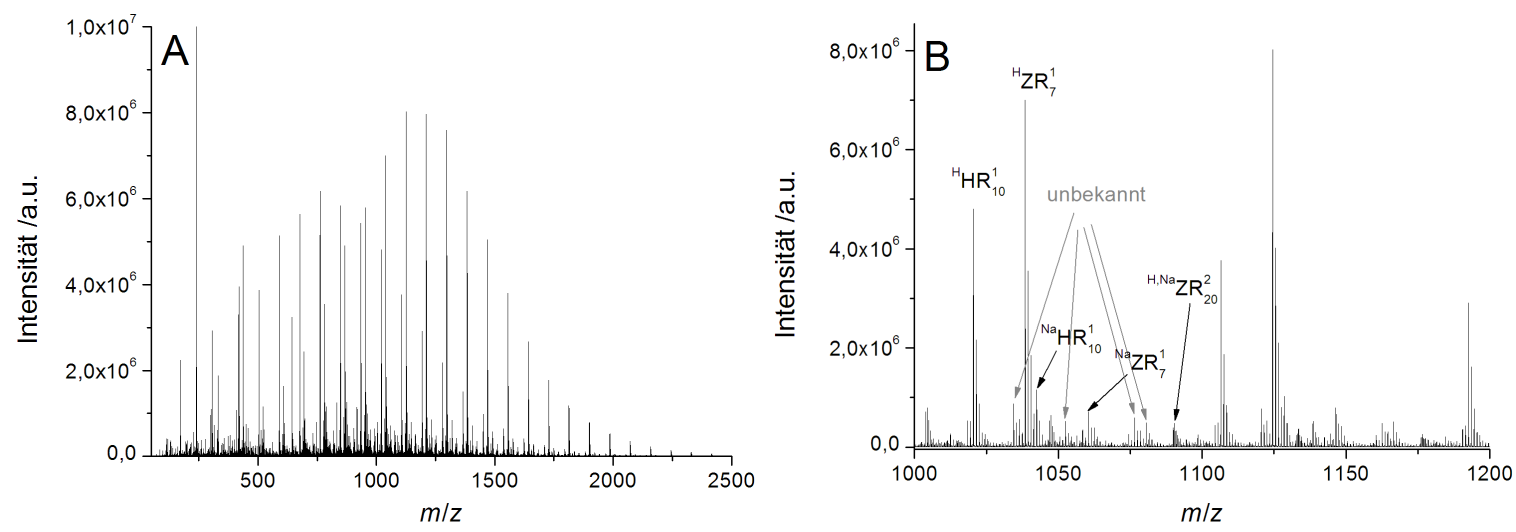

A3. Das komplette (A) ESI-Massenspektrum und ein Ausschnitt (B) des Spektrums von RAFT$\mathrm{PMA}_{1 \mathrm{k}}$-Amin gemessen in Aceton mit 0,1 wt\% Trifluoressigsäure.
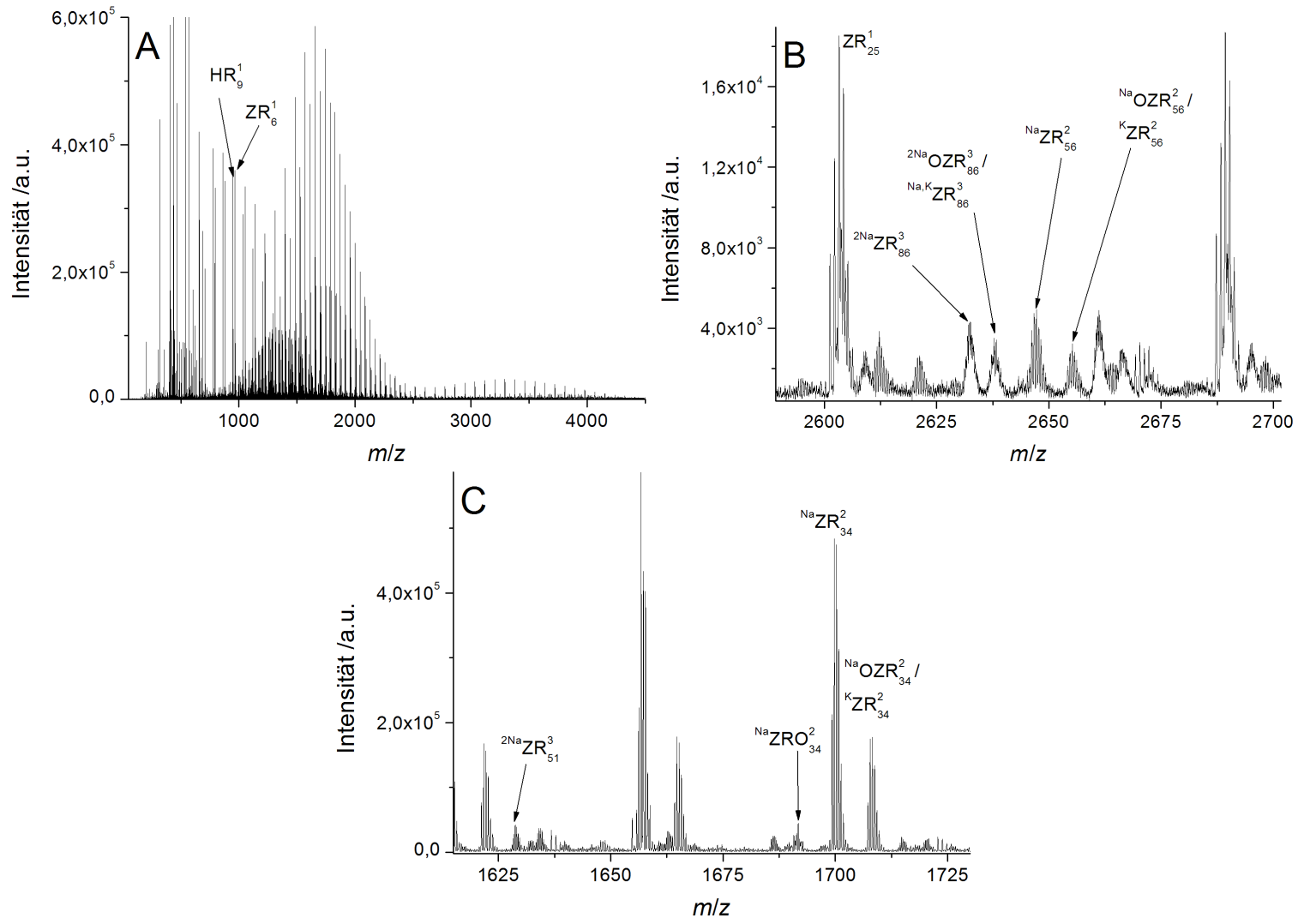

A4. Das komplette (A) ESI-Massenspektrum und Ausschnitte (B bis $C$ ) des Spektrums von RAFT-PMA $3 k^{-} \mathrm{N}^{+}$gemessen in Aceton. 

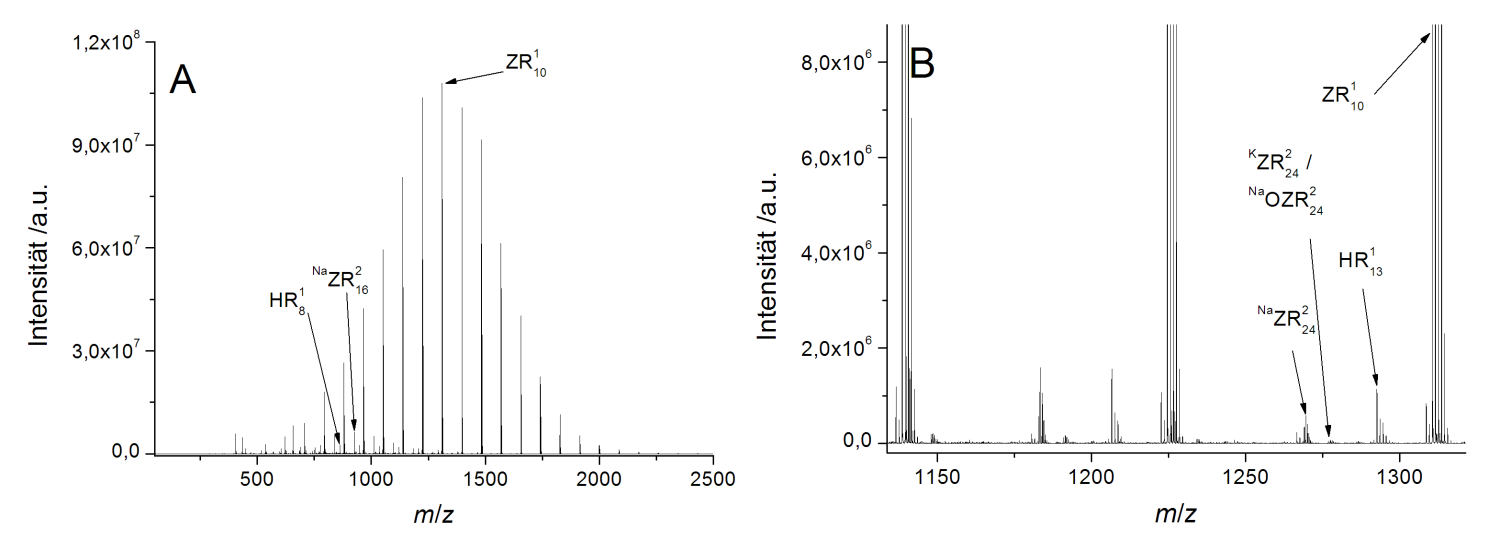

A5. Das komplette (A) ESI-Massenspektrum und ein Ausschnitt (B) des Spektrums von RAFT$\mathrm{PMA}_{1 \mathrm{k}}-\mathrm{N}^{+}$gemessen in Aceton.
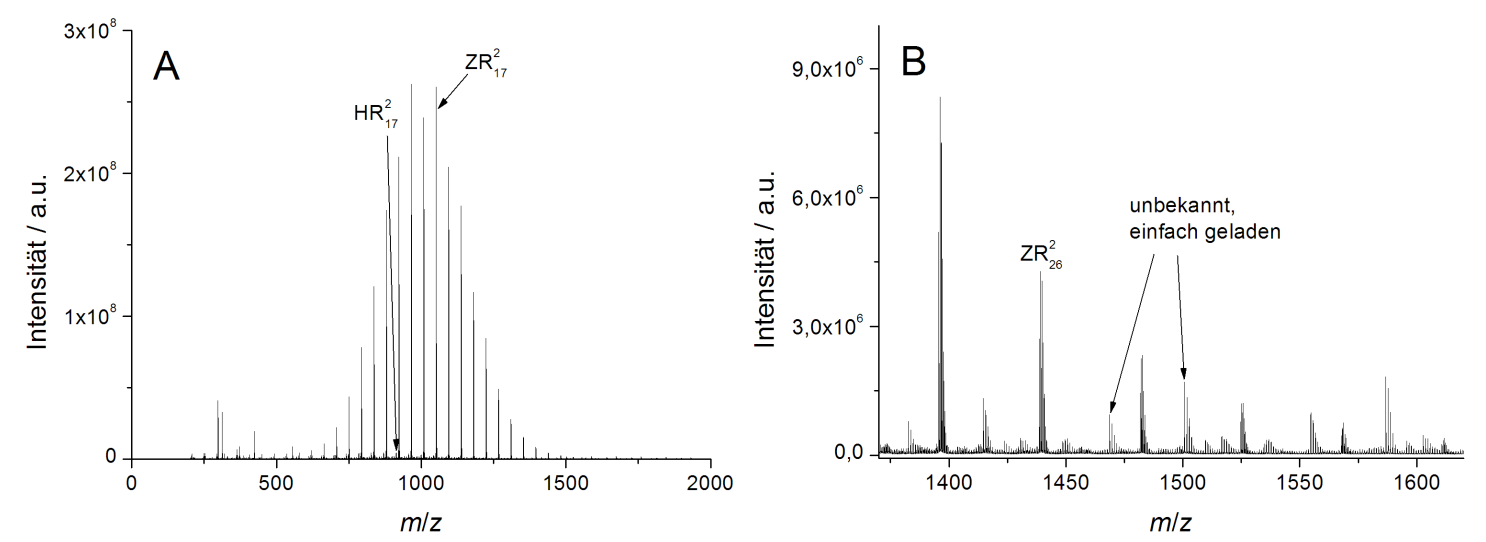

A6. Das komplette (A) ESI-Massenspektrum und ein Ausschnitt (B) des Spektrums von RAFT$\mathrm{PMA}_{2 \mathrm{k}}-\mathrm{N}^{2+}$. Die Kapillarspannung betrug $4,5 \mathrm{kV}$.
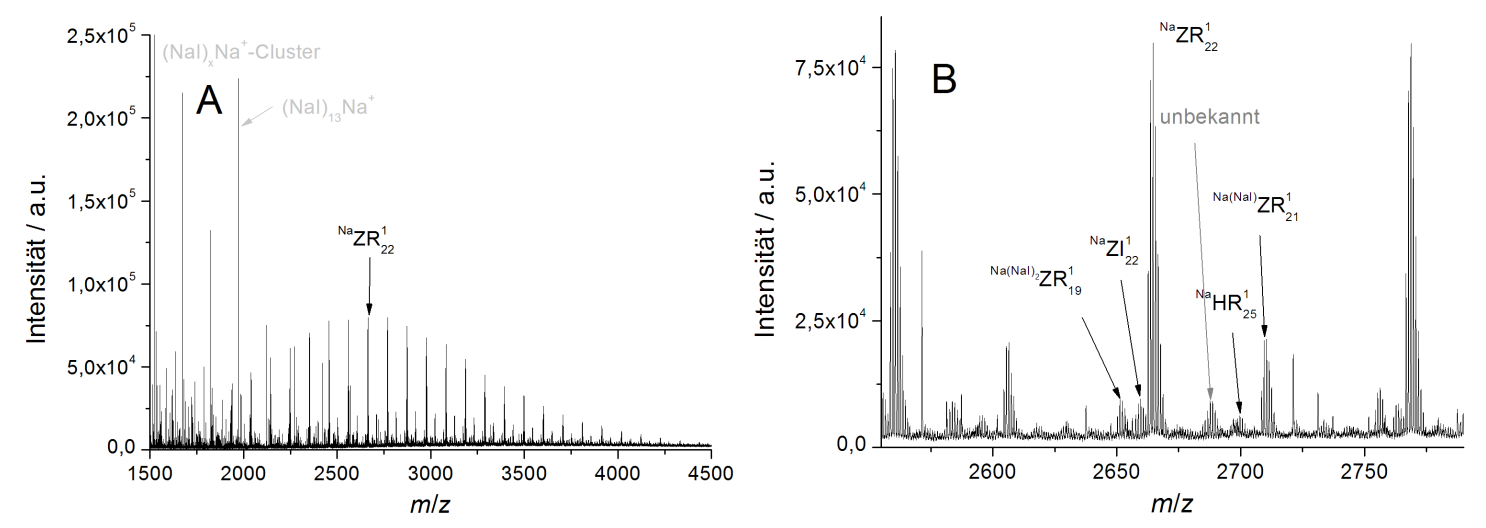

A7. Das komplette (A) ESI-Massenspektrum und ein Ausschnitt (B) des Spektrums von RAFT$\mathrm{PS}_{3 \mathrm{k}}-\mathrm{COOH}$ gemessen in Aceton mit 10 eq Nal. Die Kapillarspannung betrug 4,5 kV. 

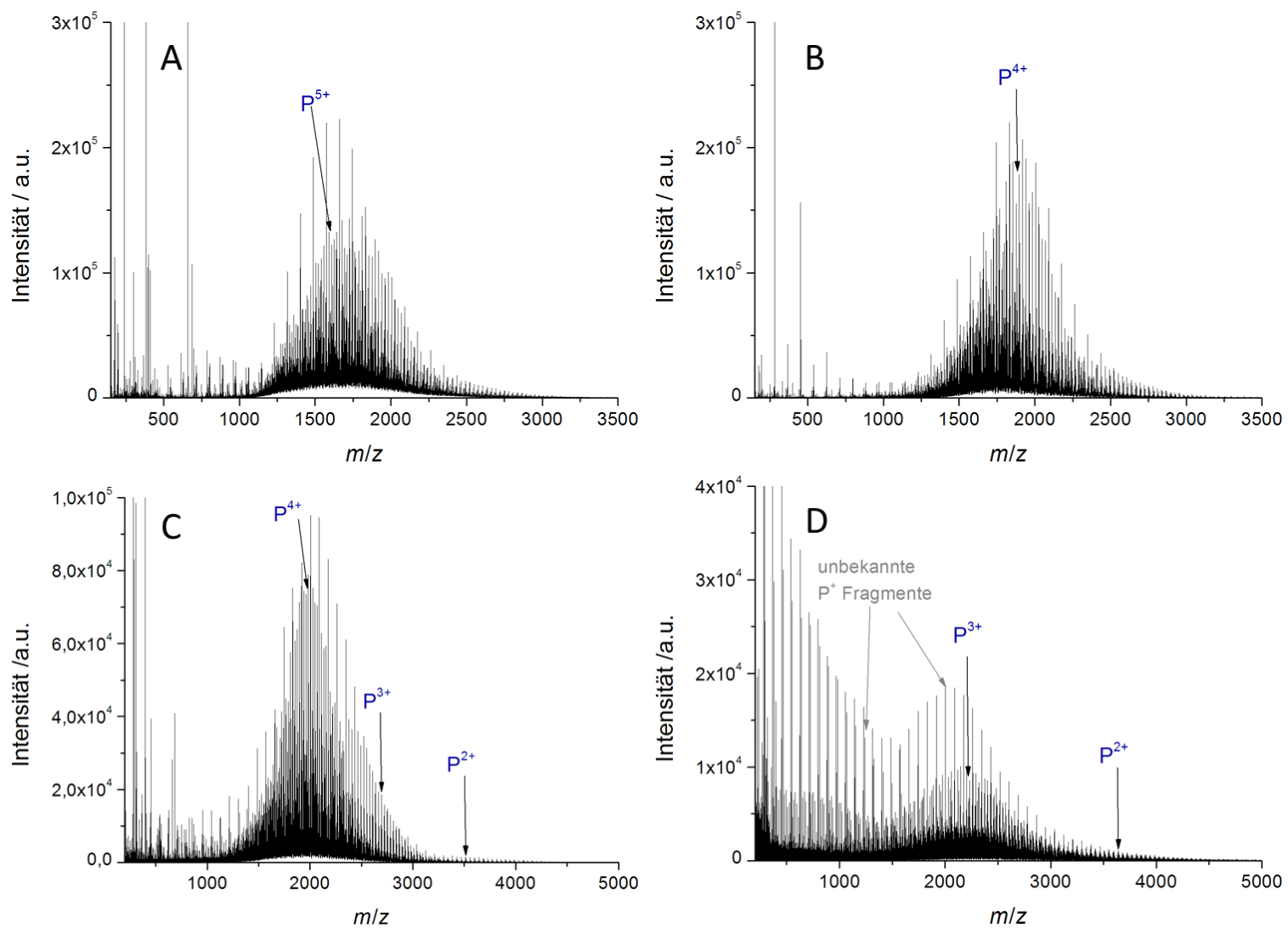

A8. Das ESI-Massenspektren einer $50 \mu \mathrm{M}$ Lösung von RAFT-PMA $A_{7 k}-\mathrm{COOH}$ mit 10 Äquivalenten $\mathrm{Nal}(\mathrm{A}, \mathrm{B})$ und ohne Zugabe von Nal (C, D). Die Stoßenergie betrug $0 \mathrm{~V}(\mathrm{~A}), 70 \mathrm{~V}(\mathrm{~B}, \mathrm{C})$ und 120 $\mathrm{V}(\mathrm{F})$.
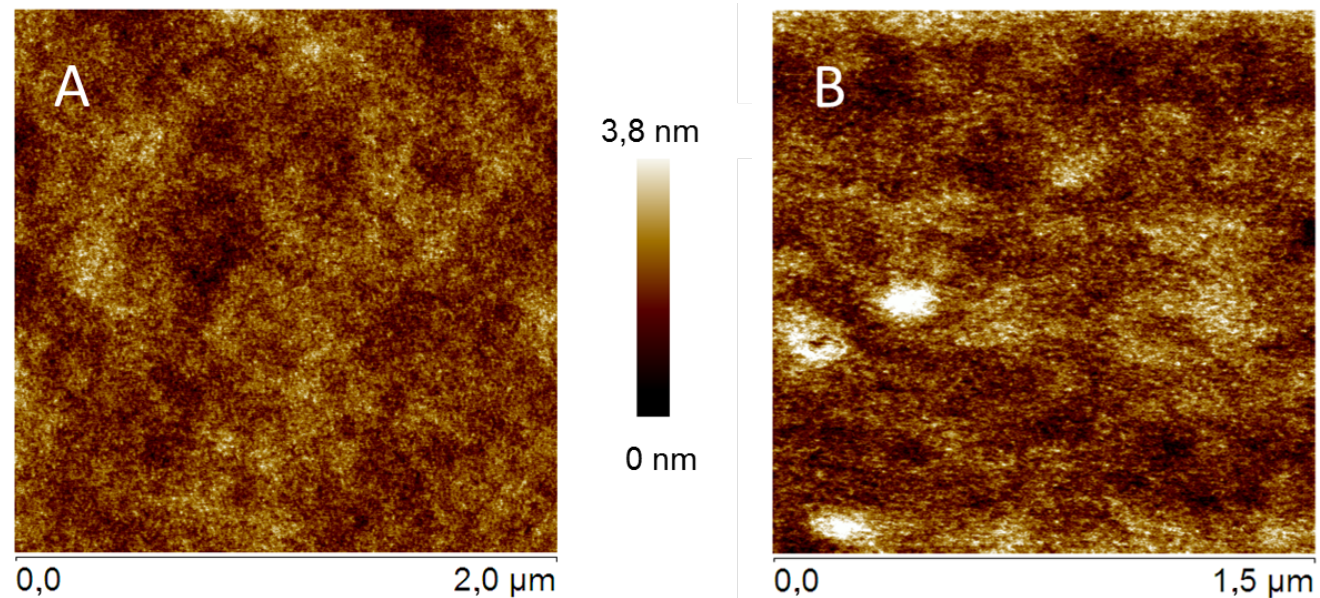

$3,2 \mathrm{~nm}$

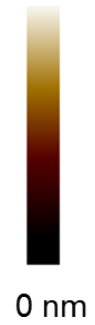

$0 \mathrm{~nm}$

A9. Topographische AFM-Aufnahmen der BCP $P(\text { LMA-co-ANMA })_{21 k}-P(B M A-c o-A N M A)_{10 k}(A)$ und $\mathrm{P}(\mathrm{LMA}-\mathrm{Co}-\mathrm{ANMA})_{21 \mathrm{k}}-\mathrm{P}(\mathrm{BMA}-\mathrm{Co}-\mathrm{ANMA})_{27 \mathrm{k}}(\mathrm{B})$ 


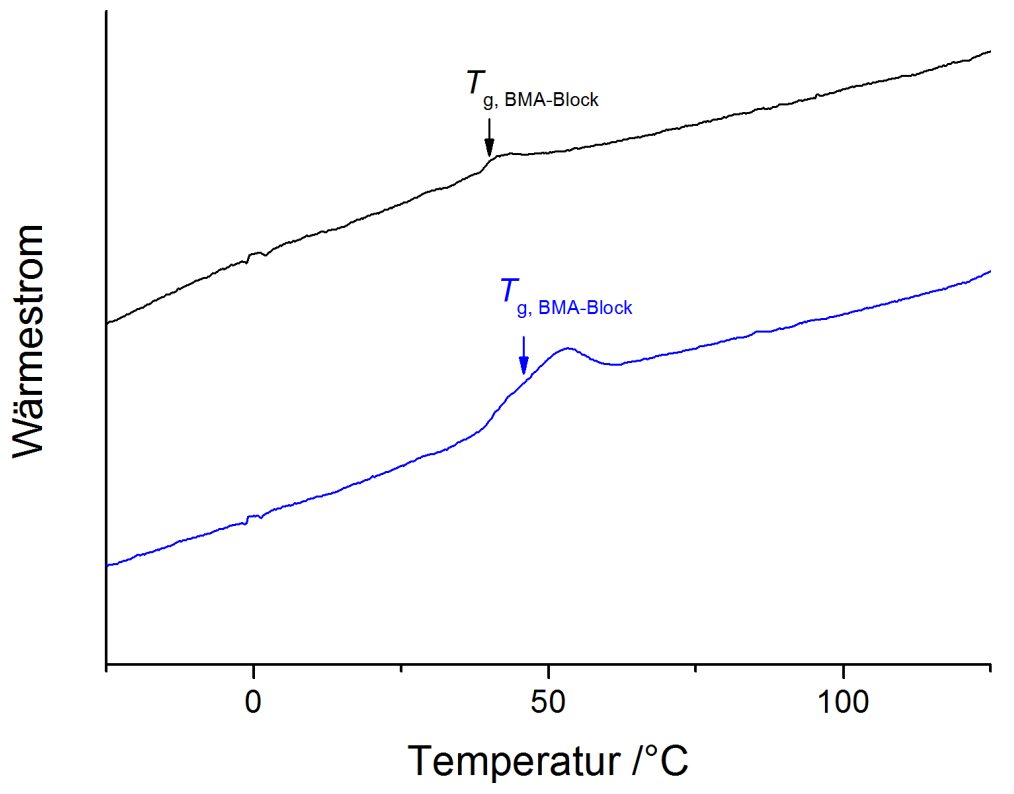

A10. DSC-Kurven der Blockcopolymere $\mathrm{P}\left(\mathrm{BMA}-\right.$-co-MMA) ${ }_{10 \mathrm{k}}-\mathrm{P}\left(\mathrm{LMA}-\right.$ co-ANMA) ${ }_{19 k}$ (schwarz) und PLMA ${ }_{21 \mathrm{k}}-\mathrm{P}(\mathrm{BMA}-\mathrm{Co}-\mathrm{ANMA})_{21 \mathrm{k}}$ (blau).

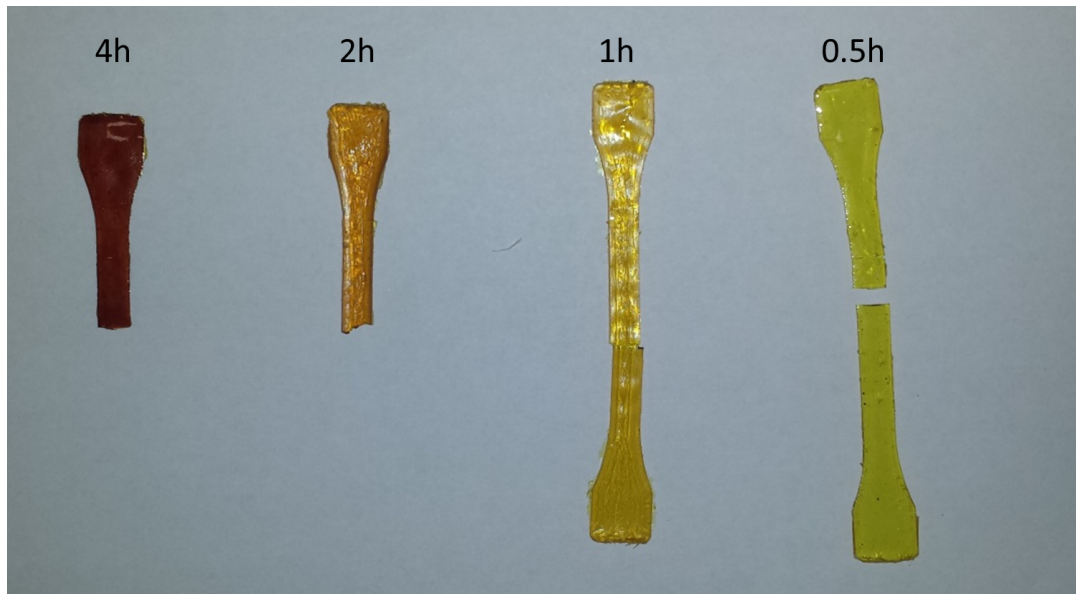

A11. Aufnahmen der unterschiedlich lang bestrahlten Probenkörper des Polymers $P\left(L_{M A} A_{0,85}\right.$ co-ANMA ${ }_{0,15}$ ) nach dem Zugversuch. 


\section{Anhang B: Tabellen}

Verweise auf Tabellen in diesem Anhang werden im Text mit dem Präfix B gekennzeichnet.

B1. Summenformeln, berechnete und experimentelle $m / z$-Werte des häufigsten Isotopenpeaks der detektierten Spezies im ESI-Massenspektrum von RAFT-PMA ${ }_{1 k}$-Amin (Abb. A3). R entspricht $-\mathrm{CH}\left(\mathrm{CH}_{3}\right) \mathrm{COO}\left(\mathrm{CH}_{2}\right)_{3} \mathrm{~N}\left(\mathrm{CH}_{3}\right)_{2}$.

\begin{tabular}{clccc}
\hline Spezies & \multicolumn{1}{c}{ Summenformel } & $\begin{array}{c}\mathrm{m} / \mathrm{z} \\
\text { berechnet }\end{array}$ & $\begin{array}{c}\mathrm{m} / \mathrm{z} \\
\text { gefunden }\end{array}$ & $\Delta^{\sqrt{a} / \mathrm{ppm}}$ \\
\hline${ }^{\mathrm{H} Z \mathrm{ZR}^{1}}{ }_{7}$ & $\mathrm{C}_{49} \mathrm{H}_{84} \mathrm{NO}_{16} \mathrm{~S}_{3}{ }^{+}$ & 1038,4947 & 1038,4917 & -3 \\
$\mathrm{H}_{\mathrm{HR}}{ }_{10}$ & $\mathrm{C}_{48} \mathrm{H}_{78} \mathrm{NO}_{22}{ }^{+}$ & 1020,5010 & 1020,4963 & -5 \\
$\mathrm{NaZl}^{1}{ }_{3}$ & $\mathrm{C}_{29} \mathrm{H}_{49} \mathrm{NO}_{6} \mathrm{~S}_{3} \mathrm{Na}^{+}$ & 626,2620 & 626,2643 & 4 \\
$\mathrm{Na}^{\mathrm{HR}}{ }_{10}^{1}$ & $\mathrm{C}_{48} \mathrm{H}_{77} \mathrm{NO}_{22} \mathrm{Na}^{+}$ & 1042,4829 & 1042,4760 & -7 \\
$\mathrm{NaZR}^{1}{ }_{7}$ & $\mathrm{C}_{49} \mathrm{H}_{83} \mathrm{NO}_{16} \mathrm{~S}_{3} \mathrm{Na}^{+}$ & 1060,4829 & 1060,4784 & -4 \\
$\mathrm{H}^{\mathrm{Na}} \mathrm{ZR}^{2}{ }_{20}$ & $\mathrm{C}_{101} \mathrm{H}_{162} \mathrm{NO}_{42} \mathrm{~S}_{3} \mathrm{Na}^{2+}$ & 1090,4827 & 1090,4791 & -3 \\
\hline
\end{tabular}

${ }^{a}$ Abweichung der epxerimentell gefundenen von den berechneten Werten.

B2. Summenformeln, berechnete und experimentelle $m / z$-Werte des häufigsten Isotopenpeaks der detektierten Spezies im ESI-Massenspektrum von RAFT-PMA ${ }_{3 \mathrm{k}}-\mathrm{N}^{+}$(Abb. A4). R entspricht $-\mathrm{CH}\left(\mathrm{CH}_{3}\right) \mathrm{COO}\left(\mathrm{CH}_{2}\right)_{3} \mathrm{~N}^{+}\left(\mathrm{CH}_{3}\right)_{3}$.

\begin{tabular}{|c|c|c|c|c|}
\hline Spezies & Summenformel & $\begin{array}{c}m / z \\
\text { berechnet }\end{array}$ & $\begin{array}{l}m / z \\
\text { gefunden }\end{array}$ & $\Delta^{a / p p m}$ \\
\hline $\mathrm{ZR}_{33}^{1}$ & $\mathrm{C}_{154} \mathrm{H}_{242} \mathrm{NO}_{68} \mathrm{~S}_{3}{ }^{+}$ & 3290,4699 & 3290,6572 & -56 \\
\hline $\mathrm{ZR}^{1}{ }_{25}$ & $\mathrm{C}_{122} \mathrm{H}_{194} \mathrm{NO}_{52} \mathrm{~S}_{3}{ }^{+}$ & 2602,1757 & 2602,2424 & 25 \\
\hline $\mathrm{ZR}_{6}^{1}$ & $\mathrm{C}_{46} \mathrm{H}_{80} \mathrm{NO}_{14} \mathrm{~S}_{3}^{+}$ & 966,4735 & 966,4747 & 1 \\
\hline $\mathrm{HR}^{1}{ }_{9}$ & $\mathrm{C}_{45} \mathrm{H}_{74} \mathrm{NO}_{20}^{+}$ & 948,4799 & 948,4838 & 4 \\
\hline $\mathrm{NaZR}^{2}{ }_{24}$ & $\mathrm{C}_{118} \mathrm{H}_{188} \mathrm{NO}_{50} \mathrm{~S}_{3} \mathrm{Na}^{2+}$ & 1269,5641 & 1269,5760 & 9 \\
\hline $\mathrm{K}_{\mathrm{ZR}}{ }^{2}{ }_{24}$ & $\mathrm{C}_{118} \mathrm{H}_{188} \mathrm{NO}_{50} \mathrm{~S}_{3} \mathrm{~K}^{2+}$ & 1277,551 & 1277,5675 & 12 \\
\hline${ }^{\mathrm{NaOZR}}{ }_{24}$ & $\mathrm{C}_{118} \mathrm{H}_{188} \mathrm{NO}_{51} \mathrm{~S}_{3} \mathrm{Na}^{2+}$ & 1277,5615 & 1277,5675 & 5 \\
\hline $\mathrm{NaZOR}^{2}{ }_{24}$ & $\mathrm{C}_{158} \mathrm{H}_{248} \mathrm{NO}_{71} \mathrm{~S}_{2} \mathrm{Na}^{2+}$ & 1691,7594 & 1691,7512 & -5 \\
\hline $2 \mathrm{NaZR}^{3}{ }_{39}$ & $\mathrm{C}_{178} \mathrm{H}_{278} \mathrm{NO}_{80} \mathrm{~S}_{3} \mathrm{Na}_{2}{ }^{3+}$ & 1284,5575 & 1284,5667 & 7 \\
\hline $\mathrm{K}, \mathrm{Na} \mathrm{ZR}^{3}{ }_{39}$ & $\mathrm{C}_{178} \mathrm{H}_{278} \mathrm{NO}_{80} \mathrm{~S}_{3} \mathrm{NaK}^{3+}$ & 1289,8821 & 1289,8915 & 7 \\
\hline${ }^{2 \mathrm{Na}} \mathrm{OZR}^{3}{ }_{39}$ & $\mathrm{C}_{178} \mathrm{H}_{278} \mathrm{NO}_{81} \mathrm{~S}_{3} \mathrm{Na}_{2}{ }^{3+}$ & 1289,8891 & 1289,8915 & 2 \\
\hline
\end{tabular}

${ }^{a} A b w e i c h u n g$ der epxerimentell gefundenen von den berechneten Werten. 
B3. Summenformeln, berechnete und experimentelle $\mathrm{m} / \mathrm{z}$-Werte des häufigsten Isotopenpeaks der detektierten Spezies im ESI-Massenspektrum von RAFT-PMA $\mathrm{k}^{-} \mathrm{N}^{+}$(Abb. A5). R entspricht $-\mathrm{CH}\left(\mathrm{CH}_{3}\right) \mathrm{COO}\left(\mathrm{CH}_{2}\right)_{3} \mathrm{~N}^{+}\left(\mathrm{CH}_{3}\right)_{3}$.

\begin{tabular}{clccc}
\hline Spezies & \multicolumn{1}{c}{ Summenformel } & $\begin{array}{c}\mathrm{m} / \mathrm{z} \\
\text { berechnet }\end{array}$ & $\begin{array}{c}\mathrm{m} / \mathrm{z} \\
\text { gefunden }\end{array}$ & $4^{\sqrt{a} / \mathrm{ppm}}$ \\
\hline $\mathrm{ZR}^{1}{ }_{10}$ & $\mathrm{C}_{62} \mathrm{H}_{104} \mathrm{NO}_{22} \mathrm{~S}_{3}{ }^{+}$ & 1310,6207 & 1310,6204 & $-0,2$ \\
$\mathrm{HR}^{1}{ }_{13}$ & $\mathrm{C}_{61} \mathrm{H}_{98} \mathrm{NO}_{28}^{+}$ & 1292,6270 & 1292,6218 & -4 \\
$\mathrm{Na}^{+} R^{2}{ }_{24}$ & $\mathrm{C}_{118} \mathrm{H}_{188} \mathrm{NO}_{50} \mathrm{~S}_{3} \mathrm{Na}^{2+}$ & 1269,5641 & 1269,5605 & -3 \\
$\mathrm{NaOZR}^{2}{ }_{24}$ & $\mathrm{C}_{118} \mathrm{H}_{188} \mathrm{NO}_{51} \mathrm{~S}_{3} \mathrm{Na}^{2+}$ & 1277,5615 & 1277,5675 & 5 \\
$\mathrm{~K}_{Z} \mathrm{R}_{24}^{2}$ & $\mathrm{C}_{118} \mathrm{H}_{188} \mathrm{NO}_{50} \mathrm{~S}_{3} \mathrm{~K}^{2+}$ & 1277,5510 & 1277,5675 & 13 \\
\hline
\end{tabular}

${ }^{a}$ Abweichung der experimentell gefundenen Werte von den berechneten Werten.

B4. Summenformeln, berechnete und experimentelle $\mathrm{m} / \mathrm{z}$-Werte des häufigsten Isotopenpeaks der detektierten Spezies im ESI-Massenspektrum von RAFT-PMA $2 \mathrm{k}-\mathrm{N}^{2+}$ (Abb. A6.

\begin{tabular}{llccc}
\hline Spezies & Summenformel & $\begin{array}{c}\mathrm{m} / \mathrm{z} \\
\text { berechnet }\end{array}$ & $\begin{array}{c}\mathrm{m} / \mathrm{z} \\
\text { gefunden }\end{array}$ & $\Delta^{\sqrt{a} / \mathrm{ppm}}$ \\
\hline $\mathrm{ZR}^{2}{ }_{17}$ & $\mathrm{C}_{103} \mathrm{H}_{166} \mathrm{~N}_{2} \mathrm{O}_{36} \mathrm{~S}_{3}{ }^{2+}$ & 1052,0270 & 1052,0202 & -6 \\
$\mathrm{HR}^{2}{ }_{17}$ & $\mathrm{C}_{90} \mathrm{H}_{142} \mathrm{~N}_{2} \mathrm{O}_{36}{ }^{2+}$ & 913,4666 & 913,4666 & 0 \\
\hline
\end{tabular}

${ }^{a}$ Abweichung der experimentell gefundenen von den berechneten Werten.

B5. Summenformeln, berechnete und experimentelle $\mathrm{m} / \mathrm{z}$-Werte des häufigsten Isotopenpeaks der detektierten Spezies im ESI-Massenspektrum von RAFT-PS ${ }_{2 k}-\mathrm{COOH}$ (Abb. 4.6). R entspricht $-\mathrm{CH}\left(\mathrm{CH}_{3}\right) \mathrm{COOH}$.

\begin{tabular}{clccc}
\hline Spezies & Summenformel & $\begin{array}{c}\mathrm{m} / \mathrm{z} \\
\text { berechnet }\end{array}$ & $\begin{array}{c}\mathrm{m} / \mathrm{z} \\
\text { gefunden }\end{array}$ & $\mathrm{A}^{\text {a }} / \mathrm{ppm}$ \\
\hline $\mathrm{NaZR}^{1}{ }_{12}$ & $\mathrm{C}_{112} \mathrm{H}_{126} \mathrm{O}_{2} \mathrm{~S}_{3} \mathrm{Na}^{+}$ & 1622,8846 & 1622,8898 & 3 \\
$\mathrm{NaZOR}^{1}{ }_{11}$ & $\mathrm{C}_{104} \mathrm{H}_{118} \mathrm{O}_{3} \mathrm{~S}_{2} \mathrm{Na}^{+}$ & 1502,8448 & 1502,8503 & 4 \\
$\mathrm{NaZl}_{11}{ }_{11}$ & $\mathrm{C}_{105} \mathrm{H}_{119} \mathrm{NS}_{3} \mathrm{Na}^{+}$ & 1513,8430 & 1513,8348 & -5 \\
$\mathrm{Na}(\mathrm{Nal}) \mathrm{ZR}^{1}{ }_{10}$ & $\mathrm{C}_{96} \mathrm{H}_{110} \mathrm{O}_{2} \mathrm{~S}_{3} \mathrm{INa}^{+}$ & 1564,6536 & 1564,6580 & 3 \\
\hline
\end{tabular}

${ }^{a}$ Abweichung der experimentell gefundenen von den berechneten Werten. 
B6. Summenformeln, berechnete und experimentelle $m / z$-Werte des häufigsten Isotopenpeaks der detektierten Spezies im ESI-Massenspektrum von RAFT-PS ${ }_{3 k}-\mathrm{COOH}$ (Abb. A7). R entspricht $-\mathrm{CH}\left(\mathrm{CH}_{3}\right) \mathrm{COOH}$.

\begin{tabular}{clccc}
\hline Spezies & \multicolumn{1}{c}{ Summenformel } & $\begin{array}{c}\mathrm{m} / \mathrm{z} \\
\text { berechnet }\end{array}$ & $\begin{array}{c}\mathrm{m} / \mathrm{z} \\
\text { gefunden }\end{array}$ & $\Delta^{\sqrt{a} / \mathrm{ppm}}$ \\
\hline $\mathrm{NaZR}^{1}{ }_{22}$ & $\mathrm{C}_{192} \mathrm{H}_{206} \mathrm{NaO}_{2} \mathrm{~S}_{3}{ }^{+}$ & 2664,5139 & 2664,6826 & 63 \\
$\mathrm{NaZI}_{22}{ }_{22}$ & $\mathrm{C}_{193} \mathrm{H}_{207} \mathrm{NNaS}_{3}{ }^{+}$ & 2659,5350 & 2659,6824 & 55 \\
$\mathrm{Na}^{\mathrm{H} R^{1}}{ }_{25}$ & $\mathrm{C}_{203} \mathrm{H}_{206} \mathrm{NaO}_{2}{ }^{+}$ & 2700,5983 & 2700,7676 & 63 \\
$\mathrm{Na}(\mathrm{Nal}) \mathrm{ZR}^{1}{ }_{21}$ & $\mathrm{C}_{184} \mathrm{H}_{198} \mathrm{INa}_{2} \mathrm{O}_{2} \mathrm{~S}_{3}{ }^{+}$ & 2709,3422 & 2709,5088 & 61 \\
$\mathrm{Na}(\mathrm{Nal})_{2} \mathrm{ZR}_{19}^{1}$ & $\mathrm{C}_{168} \mathrm{H}_{182} \mathrm{I}_{2} \mathrm{Na}_{3} \mathrm{O}_{2} \mathrm{~S}_{3}{ }^{+}$ & 2651,1112 & 2651,2695 & 60 \\
\hline
\end{tabular}

${ }^{a}$ Abweichung der experimentell gefundenen von den berechneten Werten.

B7. Summenformeln, berechnete und experimentelle $\mathrm{m} / \mathrm{z}$-Werte des häufigsten Isotopenpeaks der detektierten Spezies im ESI-Massenspektrum von RAFT-PS ${ }_{2 \mathrm{k}}-$ Amin und RAFT-PS $_{2 \mathrm{k}^{-}}$ $\mathrm{N}^{+}$(Abb. 4.7 und 4.8). $\mathrm{R}$ entspricht - $\mathrm{CH}\left(\mathrm{CH}_{3}\right) \mathrm{COO}\left(\mathrm{CH}_{2}\right)_{3} \mathrm{~N}\left(\mathrm{CH}_{3}\right)_{2}$ bei RAFT-PS $2 \mathrm{k}-$ Amin und $\mathrm{CH}\left(\mathrm{CH}_{3}\right) \mathrm{COO}\left(\mathrm{CH}_{2}\right)_{3} \mathrm{~N}^{+}\left(\mathrm{CH}_{3}\right)_{3}$ bei RAFT-PS ${ }_{2 \mathrm{k}} \mathrm{N}^{+}$.

\begin{tabular}{cclccc}
\hline RAFT-Agens & Spezies & \multicolumn{1}{c}{ Summenformel } & $\begin{array}{c}\mathrm{m} / \mathrm{z} \\
\text { berechnet }\end{array}$ & $\begin{array}{c}\mathrm{m} / \mathrm{z} \\
\text { gefunden }\end{array}$ & $\Delta^{\sqrt{a} / \mathrm{ppm}}$ \\
\hline RAFT-Amin & $\mathrm{HZR}^{1}{ }_{10}$ & $\mathrm{C}_{101} \mathrm{H}_{122} \mathrm{NO}_{2} \mathrm{~S}_{3}{ }^{+}$ & 1477,8671 & 1477,8538 & -9 \\
\hline RAFT-N $^{+}$ & $\mathrm{ZR}^{1}{ }_{8}$ & $\mathrm{C}_{86} \mathrm{H}_{108} \mathrm{NO}_{2} \mathrm{~S}_{3}{ }^{+}$ & 1282,7542 & 1282,7582 & 3 \\
RAFT-N $^{+}$ & $\mathrm{HR}^{1}{ }_{11}$ & $\mathrm{C}_{97} \mathrm{H}_{108} \mathrm{NO}_{2}{ }^{+}$ & 1318,8380 & 1318,8352 & -2 \\
RAFT-N $^{+}$ & $\mathrm{RR}^{2}{ }_{21}$ & $\mathrm{C}_{186} \mathrm{H}_{206} \mathrm{~N}_{2} \mathrm{O}_{4}{ }^{2+}$ & 1266,8023 & 1266,8091 & 5 \\
RAFT-N $^{+}$ & $2 \mathrm{ZR}^{2}{ }_{35}$ & $\mathrm{C}_{324} \mathrm{H}_{368} \mathrm{~N}_{2} \mathrm{O}_{4} \mathrm{~S}_{6}{ }^{2+}$ & 2272,8540 & 2272,9192 & 29 \\
RAFT-N $^{+}$ & $3 Z \mathrm{R}^{3}{ }_{56}$ & $\mathrm{C}_{514} \mathrm{H}_{580} \mathrm{~N}_{3} \mathrm{O}_{6} \mathrm{~S}_{9}{ }^{3+}$ & 2394,4275 & 2394,4373 & 4 \\
\hline
\end{tabular}

${ }^{a}$ Abweichung der experimentell gefundenen von den berechneten Werten.

B8. Summenformeln, berechnete und experimentelle $m / z$-Werte des häufigsten Isotopenpeaks der detektierten Spezies im ESI-Massenspektrum von RAFT-PMA ${ }_{4 k}-\mathrm{COOH}$ mit 5 und 10 äquivalenten $\mathrm{Nal}$ (Abb. 4.10). R entspricht $-\mathrm{CH}\left(\mathrm{CH}_{3}\right) \mathrm{COOH}$.

\begin{tabular}{ccccc}
\hline Spezies & Summenformel & $\begin{array}{c}\mathrm{m} / \mathrm{z} \\
\text { berechnet }\end{array}$ & $\begin{array}{c}\mathrm{m} / \mathrm{z} \\
\text { gefunden }\end{array}$ & $\mathrm{A}^{\mathrm{a}} / \mathrm{ppm}$ \\
\hline $\mathrm{Na}_{2}(\mathrm{Nal}) \mathrm{ZR}^{2}{ }_{36}$ & $\mathrm{C}_{160} \mathrm{H}_{246} \mathrm{O}_{74} \mathrm{~S}_{3} \mathrm{Na}_{3} \mathrm{I}^{2+}$ & 1822,6721 & 1822,6669 & -3 \\
$\mathrm{Na}_{2}(\mathrm{Nal})_{2} \mathrm{ZR}^{2}{ }_{3}$ & $\mathrm{C}_{156} \mathrm{H}_{240} \mathrm{O}_{72} \mathrm{~S}_{3} \mathrm{Na}_{4} \mathrm{I}_{2}{ }^{2+}$ & 1854,0992 & 1854,0883 & -6 \\
\hline
\end{tabular}

${ }^{a}$ Abweichung der experimentell gefundenen von den berechneten Werten. 
B9. Summenformeln, berechnete und experimentelle $\mathrm{m} / \mathrm{z}$-Werte des häufigsten Isotopenpeaks der einer Kapillarspannung von $5 \mathrm{kV}$ neu detektierten Spezies im ESI-Massenspektrum von RAFT-PMA ${ }_{1 k}-\mathrm{N}^{+}$(Abb. A5). R entspricht - $\mathrm{CH}\left(\mathrm{CH}_{3}\right) \mathrm{COO}\left(\mathrm{CH}_{2}\right)_{3} \mathrm{~N}^{+}\left(\mathrm{CH}_{3}\right)_{3}$.

\begin{tabular}{ccccc}
\hline Spezies & Summenformel & $\begin{array}{c}\mathrm{m} / \mathrm{z} \\
\text { berechnet }\end{array}$ & $\begin{array}{c}\mathrm{m} / \mathrm{z} \\
\text { gefunden }\end{array}$ & $\Delta^{\sqrt{a} / \mathrm{ppm}}$ \\
\hline $\mathrm{HOOR}^{1}{ }_{12}$ & $\mathrm{C}_{57} \mathrm{H}_{92} \mathrm{NO}_{28}{ }^{+}$ & 1238,5800 & 1238,5833 & 3 \\
AcR1112 $_{12}$ & $\mathrm{C}_{60} \mathrm{H}_{96} \mathrm{NO}_{27}{ }^{+}$ & 1262,6164 & 1262,6178 & 1 \\
\hline
\end{tabular}

${ }^{a}$ Abweichung der experimentell gefundenen Werte von den berechneten Werten.

B10. Summenformeln, berechnete und experimentelle $m / z$-Werte des häufigsten Isotopenpeaks von unterschiedlich langen Ketten der ZR Spezies der RAFT-PMA ${ }_{7 k}-\mathrm{N}^{+}$Probe (Abb. 4.19). $\mathrm{R}$ entspricht - $\mathrm{CH}\left(\mathrm{CH}_{3}\right) \mathrm{COO}\left(\mathrm{CH}_{2}\right)_{3} \mathrm{~N}^{+}\left(\mathrm{CH}_{3}\right)_{3}$.

\begin{tabular}{ccccc}
\hline Spezies & Summenformel & $\begin{array}{c}\mathrm{m} / z \\
\text { berechnet }\end{array}$ & $\begin{array}{c}\mathrm{m} / \mathrm{z} \\
\text { gefunden }\end{array}$ & $\left.\Delta /\left(m z^{-1}\right)^{a}\right]^{a}$ \\
\hline $\mathrm{ZR}^{1}{ }_{25}$ & $\mathrm{C}_{122} \mathrm{H}_{194} \mathrm{NO}_{52} \mathrm{~S}_{3}{ }^{+}$ & 2602,1757 & 2602,2424 & 0,0667 \\
$\mathrm{ZR}^{1}{ }_{40}$ & $\mathrm{C}_{182} \mathrm{H}_{284} \mathrm{NO}_{82} \mathrm{~S}_{3}{ }^{+}$ & 3892,7274 & 3893,9346 & 1,2072 \\
$\mathrm{ZR}^{1}{ }_{58}$ & $\mathrm{C}_{254} \mathrm{H}_{392} \mathrm{NO}_{118} \mathrm{~S}_{3}{ }^{+}$ & 5443,3961 & 5446,9927 & 3,5966 \\
$\mathrm{ZR}^{1} 78$ & $\mathrm{C}_{334} \mathrm{H}_{512} \mathrm{NO}_{158} \mathrm{~S}_{3}{ }^{+}$ & 7164,1317 & 7168,3999 & 4,2682 \\
\hline
\end{tabular}

${ }^{a}$ Abweichung der experimentell gefundenen Werte von den berechneten Werten.

B11. Summenformeln, berechnete und experimentelle $m / z$-Werte des häufigsten Isotopenpeaks der RAFT-PMA-COOH-Polymere in der MALDI-MS. R entspricht - $\mathrm{CH}\left(\mathrm{CH}_{3}\right) \mathrm{COOH}$.

\begin{tabular}{|c|c|c|c|c|c|}
\hline Polymer & Spezies & Summenformel & $\begin{array}{c}m / z \\
\text { berechnet }\end{array}$ & $\begin{array}{c}m / z \\
\text { gefunden }\end{array}$ & $\Delta /\left(m z^{-1}\right)^{a}$ \\
\hline $\begin{array}{l}\mathrm{PMA}_{2 \mathrm{k}} \\
\mathrm{PMA}_{2 \mathrm{k}}\end{array}$ & $\begin{array}{l}\mathrm{NaZR}^{1}{ }_{22} \\
{ }^{\mathrm{Na}} \mathrm{ZR}^{1}{ }_{23}\end{array}$ & $\begin{array}{l}\mathrm{C}_{57} \mathrm{H}_{96} \mathrm{NO}_{20} \mathrm{~S}_{3}^{+} \\
\mathrm{C}_{108} \mathrm{H}_{167} \mathrm{O}_{48} \mathrm{~S}_{3} \mathrm{Na}_{2}^{+}\end{array}$ & $\begin{array}{l}2266,9 \\
2374,0\end{array}$ & $\begin{array}{l}2267,6 \\
2375,6\end{array}$ & $\begin{array}{l}0,7 \\
1,7\end{array}$ \\
\hline $\begin{array}{l}\mathrm{PMA}_{4 k} \sqrt{b} \\
\mathrm{PMA}_{4 \mathrm{k}}\end{array}$ & $\begin{array}{l}\mathrm{Na}_{Z} \mathrm{R}^{1}{ }_{40} \\
{ }^{2 \mathrm{Na}_{Z} \mathrm{R}^{1}}{ }_{40}\end{array}$ & $\begin{array}{l}\mathrm{C}_{176} \mathrm{H}_{270} \mathrm{O}_{82} \mathrm{~S}_{3} \mathrm{Na}^{+} \\
\mathrm{C}_{176} \mathrm{H}_{269} \mathrm{O}_{82} \mathrm{~S}_{3} \mathrm{Na}_{2}{ }^{+}\end{array}$ & $\begin{array}{l}3815,6 \\
3837,6\end{array}$ & $\begin{array}{l}3817,9 \\
3839,9\end{array}$ & $\begin{array}{l}2,3 \\
2,3\end{array}$ \\
\hline $\begin{array}{l}\mathrm{PMA}_{7 \sqrt{b}} \\
\mathrm{PMA}_{7} \sqrt{b}\end{array}$ & $\begin{array}{l}\mathrm{NaZR}^{1}{ }_{83} \\
2^{\mathrm{Na}_{Z} \mathrm{R}^{1}}{ }_{83}\end{array}$ & $\begin{array}{l}\mathrm{C}_{348} \mathrm{H}_{528} \mathrm{O}_{168} \mathrm{~S}_{3} \mathrm{Na}^{+} \\
\mathrm{C}_{348} \mathrm{H}_{527} \mathrm{O}_{168} \mathrm{~S}_{3} \mathrm{Na}_{2}{ }^{+}\end{array}$ & $\begin{array}{l}7517,2 \\
7539,2\end{array}$ & $\begin{array}{l}7522,9 \\
7544,4\end{array}$ & $\begin{array}{l}5,7 \\
5,2\end{array}$ \\
\hline
\end{tabular}

${ }^{a}$ Abweichung der experimentell ermittelten Werte von den berechneten Werten.

${ }^{b}$ Keine Auflösung des Isotopenmusters. 
B12. Summenformeln, berechnete und experimentelle $\mathrm{m} / \mathrm{z}$-Werte des häufigsten Isotopenpeaks der RAFT-PMA-Amin-Polymere in der MALDI-MS. R entspricht - $\mathrm{CH}\left(\mathrm{CH}_{3}\right) \mathrm{COOH}$.

\begin{tabular}{|c|c|c|c|c|c|}
\hline Polymer & Spezies & Summenformel & $\begin{array}{c}m / z \\
\text { berechnet }\end{array}$ & $\begin{array}{l}m / z \\
\text { gefunden }\end{array}$ & $\Delta^{a} /\left(m z^{-1}\right)$ \\
\hline $\mathrm{PMA}_{1 \mathrm{k}}$ & $\mathrm{HZR}^{1}{ }_{19}$ & $\mathrm{C}_{57} \mathrm{H}_{96} \mathrm{NO}_{20} \mathrm{~S}_{3}{ }^{+}$ & 1210,6 & 1210,7 & 0,1 \\
\hline $\begin{array}{l}\mathrm{PMA}_{3 \mathrm{k}} \\
\mathrm{PMA}_{3 \mathrm{k}}\end{array}$ & $\begin{array}{l}\mathrm{H}_{Z R^{1}}{ }_{39} \\
\mathrm{Na}_{Z} \mathrm{R}^{1}{ }_{39}\end{array}$ & $\begin{array}{l}\mathrm{C}_{177} \mathrm{H}_{276} \mathrm{NO}_{80} \mathrm{~S}_{3}^{+} \\
\mathrm{C}_{177} \mathrm{H}_{275} \mathrm{NO}_{80} \mathrm{~S}_{3} \mathrm{Na}^{+}\end{array}$ & $\begin{array}{l}3792,7 \\
3814,7\end{array}$ & $\begin{array}{l}3790,0 \\
3811,9\end{array}$ & $\begin{array}{l}-2,7 \\
-2,8\end{array}$ \\
\hline $\begin{array}{l}\mathrm{PMA}_{7 \sqrt{b}} \\
\mathrm{PMA}_{7 \mathrm{k}}\end{array}$ & $\begin{array}{l}\mathrm{H}_{Z R^{1}}{ }_{83} \\
\mathrm{Na} \mathrm{R}^{1}{ }_{83}\end{array}$ & $\begin{array}{l}\mathrm{C}_{353} \mathrm{H}_{540} \mathrm{NO}_{168} \mathrm{~S}_{3}^{+} \\
\mathrm{C}_{353} \mathrm{H}_{539} \mathrm{NO}_{168} \mathrm{~S}_{3} \mathrm{Na}^{+}\end{array}$ & $\begin{array}{l}7580,3 \\
7602,3\end{array}$ & $\begin{array}{l}7580,5 \\
7603,0\end{array}$ & $\begin{array}{l}0,2 \\
0,7\end{array}$ \\
\hline
\end{tabular}

${ }^{a}$ Abweichung der experimentell gefundenen Werte von den berechneten Werten.

${ }^{b}$ Keine Auflösung des Isotopenmusters.

B13. Summenformeln, berechnete und experimentelle $m / z$-Werte des häufigsten Isotopenpeaks der RAFT-PMA-COOH-Polymere in der MALDI-MS. R entspricht - $\mathrm{CH}\left(\mathrm{CH}_{3}\right) \mathrm{COOH}$.

\begin{tabular}{lllccc}
\hline Polymer & Spezies & \multicolumn{1}{c}{ Summenformel } & $\begin{array}{c}\mathrm{m} / \mathrm{z} \\
\text { berechnet }\end{array}$ & $\begin{array}{c}\mathrm{m} / \mathrm{z} \\
\text { gefunden }\end{array}$ & $\mathrm{A}^{\mathrm{a} /\left(m z^{-1}\right)}$ \\
\hline PMA1k & $\mathrm{ZR}^{1}{ }_{14}$ & $\mathrm{C}_{78} \mathrm{H}_{128} \mathrm{NO}_{30} \mathrm{~S}_{3}{ }^{+}$ & 1654,8 & 1654,8 & 0,0 \\
PMA1k & $\mathrm{ZR}^{1}{ }_{1}$ & $\mathrm{C}_{26} \mathrm{H}_{50} \mathrm{NO}_{4} \mathrm{~S}_{3}{ }^{+}$ & 536,3 & 536,1 & $-0,2$ \\
PMA1k & $\mathrm{HR}^{1}{ }_{4}$ & $\mathrm{C}_{25} \mathrm{H}_{44} \mathrm{NO}_{10^{+}}$ & 518,3 & 518,1 & $-0,2$ \\
\hline PMA3k & $\mathrm{ZR}^{1}{ }_{39}$ & $\mathrm{C}_{178} \mathrm{H}_{278} \mathrm{NO}_{80} \mathrm{~S}_{3}{ }^{+}$ & 3806,7 & 3802,9 & $-3,8$ \\
PMA3k & $\mathrm{ZR}^{1}{ }_{21}$ & $\mathrm{C}_{106} \mathrm{H}_{170} \mathrm{NO}_{44} \mathrm{~S}_{3}{ }^{+}$ & 2258,0 & 2257,5 & $-0,5$ \\
PMA3k & $\mathrm{HR}^{1}{ }_{24}$ & $\mathrm{C}_{105} \mathrm{H}_{164} \mathrm{NO}_{50^{+}}$ & 2240,0 & 2239,4 & $-0,6$ \\
\hline PMA7k & $\mathrm{ZR}^{1}{ }_{67}$ & $\mathrm{C}_{290} \mathrm{H}_{446} \mathrm{NO}_{136} \mathrm{~S}_{3}{ }^{+}$ & 6217,7 & 6213,6 & $-4,1$ \\
PMA7k & $\mathrm{HR}^{1}{ }_{70}$ & $\mathrm{C}_{289} \mathrm{H}_{440} \mathrm{NO}_{142}{ }^{+}$ & 6198,7 & 6195,9 & $-2,8$ \\
PMA7k & $\mathrm{ZR}^{1}{ }_{83}$ & $\mathrm{C}_{354} \mathrm{H}_{542} \mathrm{NO}_{168} \mathrm{~S}_{3}{ }^{+}$ & 7594,3 & 7590,6 & $-3,7$ \\
\hline
\end{tabular}

${ }^{a}$ Abweichung der experimentell gefundenen Werte von den berechneten Werten.

${ }^{b}$ Keine Auflösung der Isotopen.

B14. Summenformeln, berechnete und experimentelle $\mathrm{m} / \mathrm{z}$-Werte des häufigsten Isotopenpeaks der Polystyrole in der MALDI-MS. R entspricht $-\mathrm{CH}\left(\mathrm{CH}_{3}\right) \mathrm{COOH}$.

\begin{tabular}{cclccc}
\hline Polymer & Spezies & Summenformel & $\begin{array}{c}m / z \\
\text { berechnet }\end{array}$ & $\begin{array}{c}m / z \\
\text { gefunden }\end{array}$ & $\Delta^{\sqrt{ } /\left(m z^{-1}\right)}$ \\
\hline $\mathrm{PS}_{2 \mathrm{k}}-\mathrm{COOH}$ & ${ }^{2 A g U R^{1}}{ }_{11}$ & $\mathrm{C}_{91} \mathrm{H}_{91} \mathrm{O}_{2} \mathrm{Ag}_{2}{ }^{+}$ & 1431,5 & 1430,2 & $-1,3$ \\
$\mathrm{PS}_{2 \mathrm{k}}-\mathrm{COOH}$ & $\mathrm{AgUR}^{1}{ }_{12}$ & $\mathrm{C}_{99} \mathrm{H}_{100} \mathrm{O}_{2} \mathrm{Ag}^{+}$ & 1428,7 & 1430,2 & $+1,5$ \\
$\mathrm{PS}_{2 \mathrm{k}}-\mathrm{Amin}$ & $\mathrm{H}_{\mathrm{ZR}}{ }_{17}$ & $\mathrm{C}_{157} \mathrm{H}_{178} \mathrm{NO}_{2} \mathrm{~S}_{3}{ }^{+}$ & 2206,3 & 2202,4 & $-3,9$ \\
$\mathrm{PS}_{2 \mathrm{k}}-\mathrm{N}+$ & $\mathrm{ZR}^{1}{ }_{13}$ & $\mathrm{C}_{126} \mathrm{H}_{148} \mathrm{NO}_{2} \mathrm{~S}_{3}{ }^{+}$ & 1804,1 & 1804,7 & $+0,6$ \\
\hline
\end{tabular}

${ }^{a}$ Abweichung der experimentell gefundenen Werte von den berechneten Werten. 


\section{Danksagung}

Zuerst möchte ich mich bei Herrn Prof. Dr. Philipp Vana für die Überlassung des hochinteressanten Themas, hilfreichen Ratschlägen und der Möglichkeit frei an weiteren Projekten arbeiten zu dürfen bedanken. Ich möchte auch Herrn Prof. Dr. Marcus Müller für die Betreuung und die Übernahme des Korreferats sowie die vielen hilfreichen Diskussionen und Anregungen während meiner Dissertation danken. Ebenso möchte ich mich bei dem Prüfungskomitee und der Deutschen Forschungsgemeinschaft für die kontinuierliche finanzielle Unterstützung meiner Arbeit im Rahmen des SFB 937 bedanken.

Dr. Holm Frauendorf und Dr. Shinsuke Kokubo danke ich für die Einarbeitung in das MALDIbzw. ESI-Massenspektrometer. Großer Dank gilt auch Jannik Wagner für die gute Zusammenarbeit in unserem gemeinsamen Projekt, der (gegenseitigen) Motivationsreden und den immer bereitstehenden Tipps beim Schreiben meiner Dissertation, obwohl du selbst mit dem Schreibprozess deiner Arbeit beschäftigt warst. Bei folgenden Personen möchte ich mich für das Korrekturlesen ganzer Kapitel oder Ausschnitte meiner Doktorarbeit bedanken: Julia Gerke, Katharina Dabow, Dr. Martin Königsmann, Judith Steinhoff, Jannik Wagner, Annika Nitschke, Torsten Fornefeld, Stella Weber und Darius Rohleder. Zudem danke ich meinen Praktikanten Jana Rohde und Daniel Berndt, die im Rahmen ihrer Forschungspraktika Experimente für mich durchgeführt haben.

Ebenso bedanke ich mich bei allen aktuellen und ehemaligen Mitgliedern des Arbeitskreises Prof. Dr. Philipp Vana während meiner Promotion für das angenehme Arbeitsklima. Ich hoffe, dass die entstandenen Freundschaften noch lange währen werden. Vielen Dank auch an meine ehemaligen Kommilitonen Phil Pithan, Sebastian von Gradowski, Nils Steinbrück und Benedikt Steinhoff für die gegenseitige Unterstützung während und nach Beendigung des Studiums. Ich bin froh, dass wir den Kontakt trotz des ein oder anderen Umzugs gehalten haben und hoffentlich weiter halten werden.

Des Weiteren danke ich meiner Freundin Julia Gerke die - wie bereits erwähnt (dieser Einschub ist für Dich! :P) - meine Arbeit auf Rechtschreib- und Grammatikfehler hin korrekturgelesen hat und die viel Verständnis für meine mangelnde Freizeit am Ende meiner Schreibphase gezeigt hat.

Zu guter Letzt möchte ich den beiden Menschen danken, die mich seit über 29 Jahren täglich unterstützen und vor allem während des Studiums und der Promotion viel Verständnis gezeigt haben: meinen Eltern. Ohne Euch wäre sicherlich weder mein Studium noch meine Promotion möglich gewesen! 